ANL-7394

ANL-7394

251
$9 / 24 / 68$

Argonne Дational Laboratoru

ANALYSIS AND REDUCTION OF

PROTON-RECOIL DATA

by

E. F. Bennett, R. Gold, and I. K. Olson 


\section{DISCLAIMER}

This report was prepared as an account of work sponsored by an agency of the United States Government. Neither the United States Government nor any agency Thereof, nor any of their employees, makes any warranty, express or implied, or assumes any legal liability or responsibility for the accuracy, completeness, or usefulness of any information, apparatus, product, or process disclosed, or represents that its use would not infringe privately owned rights. Reference herein to any specific commercial product, process, or service by trade name, trademark, manufacturer, or otherwise does not necessarily constitute or imply its endorsement, recommendation, or favoring by the United States Government or any agency thereof. The views and opinions of authors expressed herein do not necessarily state or reflect those of the United States Government or any agency thereof. 


\section{DISCLAIMER}

Portions of this document may be illegible in electronic image products. Images are produced from the best available original document. 
The facilities of Argonne National Laboratory are owned by the United States Government. Under the terms of a contract (W-31-109-Eng-38) between the U. S. Atomic Energy Commission, Argonne Universities Association and The University of Chicago, the University employs the staff and operates the Laboratory in accordance with policies and programs formulated, approved and reviewed by the Association.

\section{MEMBERS OF ARGONNE UNIVERSITIES ASSOCIATION}

The University of Arizona

Carnegie-Mellon University

Case Western Reserve University

The University of Chicago

University of Cincinnati

Illinois Institute of Technology

University of Illinois

Indiana University

Iowa State University

The University of Iowa
Kansas State University

The University of Kansas

Loyola University

Marquette University

Michigan State University

The University of Michigan

University of Minnesota

University of Missouri

Northwestern Untversily

University of Notre Dame
The Ohio State University

Ohio University

The Pennsylvania State University

Purdue University

Saint Louis University

Southern Illinois University

University of Texas

Washinglon University

Wayie State Univeroity

The University of Wisconsin

\section{LEGAL NOTICE}

This report was prepared as an account of Government sponsored work. Neither the United States, nor the Commission, nor any person acting on behalf of the Commission:

A. Makes any warranty or representation, expressed or implied, with respect to the accuracy, completeness, or usefulness of the information contained in this report, or that the use of any information, apparatus, method, or process disclosed in this report may not infringe privately owned rights; or

B. Assumes any liabilities with respect to the use of, or for damages resulting from the use of any information, apparatus, method, or process disclosed in this report.

As used in the above, "person acting on behalf of the Commission" includes any employee or contractor of the Commission, or employee of such contractor, to the extent that such employee or contractor of the Commission, or employee of such contractor prepares, disseminates, or provides access to, any information pursuant to his employment or contract with the Commission, or his employment with such contractor.

Printed in the United States of America

Available from

Clearinghouse for Federal Scientific and Technical Information

National Bureau of Standards, U. S. Department of Commerce

Springfield, Virginia 22151

Price: Printed Copy $\$ 3.00 ;$ Microfiche $\$ 0.65$ 


\author{
ARGONNE NATIONAL LABORATORY \\ 9700 South Cass Avenue \\ Argonne, Illinois 60439
}

\title{
ANALYSIS AND REDUCTION OF PROTON-RECOIL DATA
}

by

E. F. Bennett, R. Gold, and I. K. Olson

Reactor Physics Division.

\section{LEGAL NOTICE}

This report was prepared as an account of Government sponsored work. Neither the Unlted States, nor the Commission, nor any person acting on behalf of the Commisston:

implied, with respect to the accuA. compieteness, or usefulness of the information contained in this report, or that the use of any information, apparatus, method, or process disclosed in this report may not infringe of any information, apparatud

B. Assumes any liabllitles with respect to the use of, or for damages resultung from the
privalely owned rights; or use of any information, apparatus, method, or process disclosed in this report.

As used in the above. "person acting on behalf of the Commission" includes any employee or contractor of the Commission, or employee of such contractor, to the extent that such employee or contractor of the Commlsalon, of employee of such contractor prepares, diseminates, or provides access to, any information pursuant

with the Commission, or his employment with such contri 


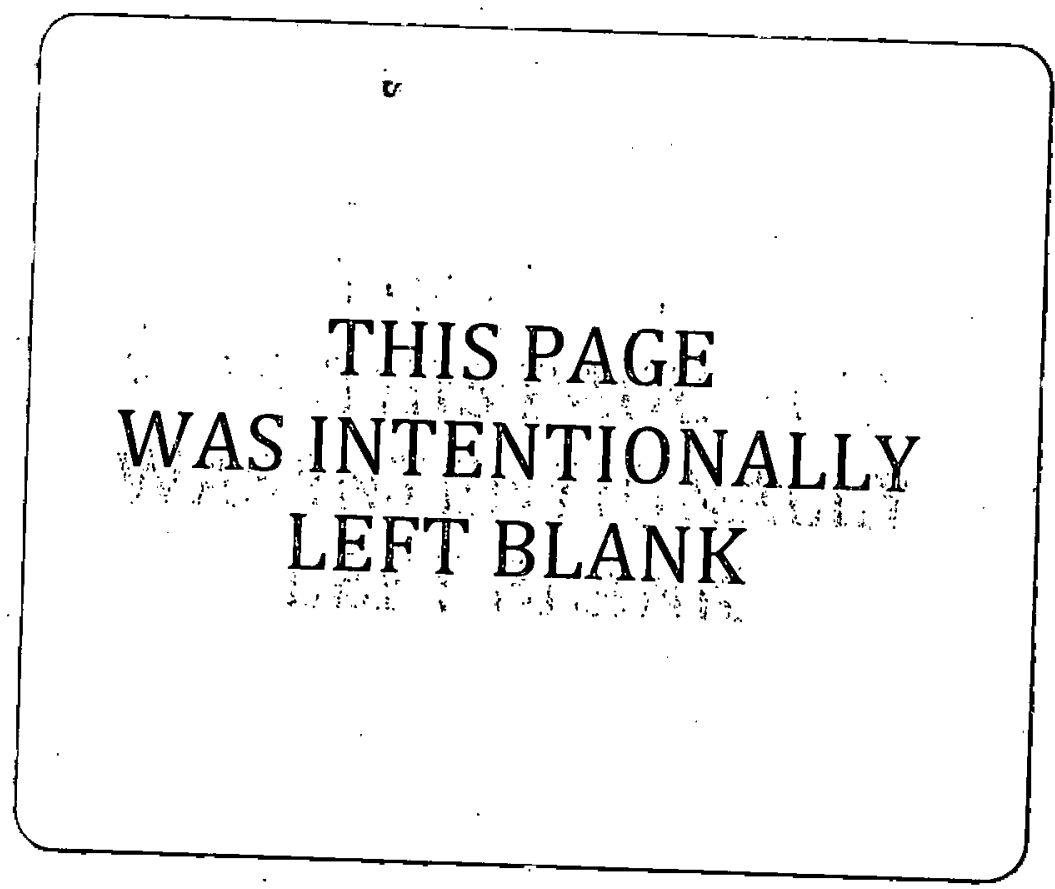


TABLE OF CONTENTS

Page

ABSTRAĊ $\ldots \ldots \ldots \ldots \ldots \ldots \ldots \ldots$

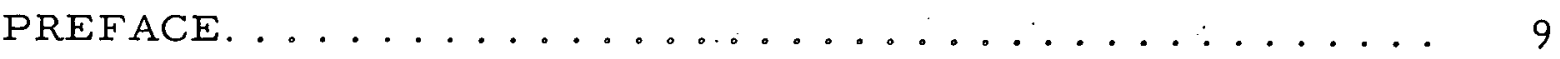

I. PROTON-RECOIL PROPORTIONAL-COUNTER RESPONSE

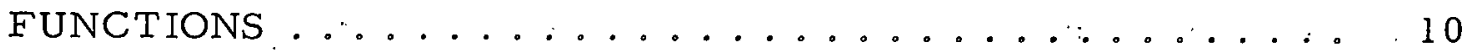

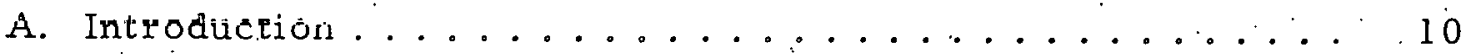

B. Energy-independent Response-function Measurements . . . . 12

C. Energy-dependent Response Function . . . . . . . . . . . . . . . .

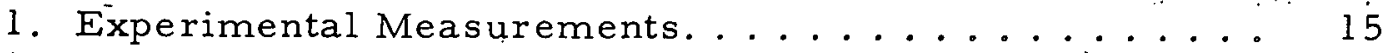

2. Energy-density Transformations ............ 20

D. Analytical Formation of the Response Function. . . . . . . 24

1. Treatment and Parametrization of Experimental Data. . 24

2. Construction of Response Matrix............ 32

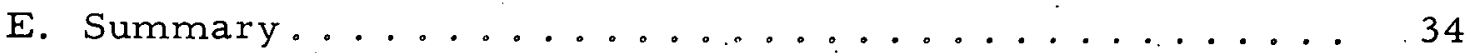

II. UNFOLDING FINITE-SIZE EFFECTS ............ 36

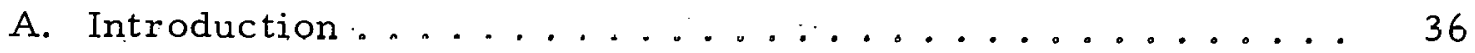

B. Iterative Unfolding of Proton-recoil Spectra........ 36

C. Integral Unfolding..................... 41

D. Comparison of Unfolding Methods.............. 42

III. REDUCTION OF PROTON-RECOIL PULSE-HEIGHT DISTRIBUTIONS TO NEUTRON-FLUX SPECTRA. . . . . . . 44

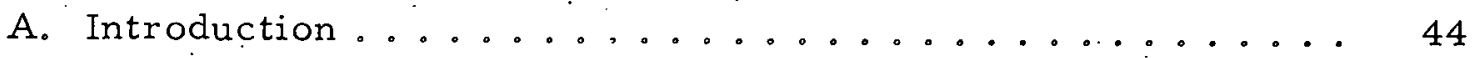

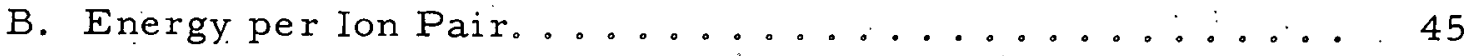

C. Carbon and Nitrogen Recoils ............... 45

IV. COMPUTER PROGRAMS ............... 47

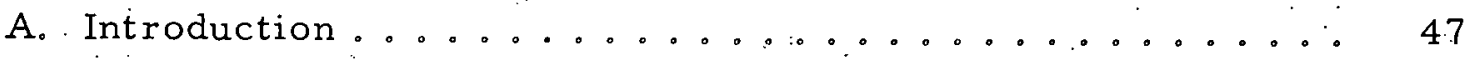

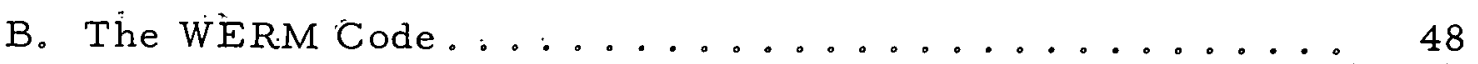

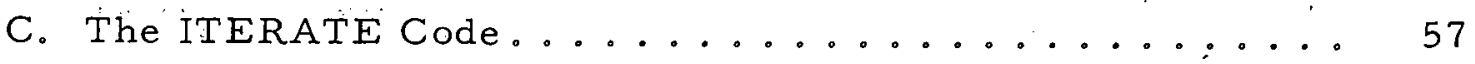


TABLE OF CONTENTS

Page

D. Discussion of PSNS Code and Test Problem......... 61

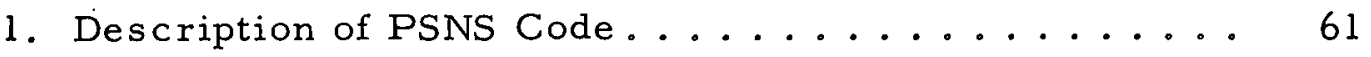

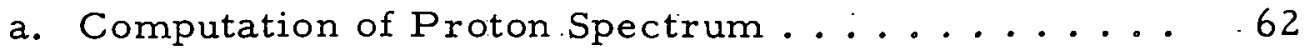

b. Integral Response Unfolding........... 62

c. Energy: Spacing and Neutron-flux Format ..... 63

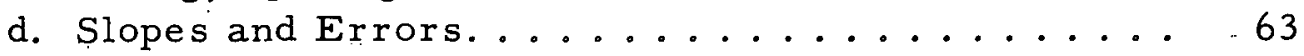

e. Heavy-particle Recoils ............... 64

f. Form of Punshed Data............. 65

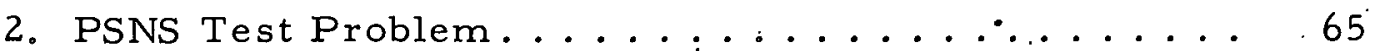

\section{APPENDIXES}

A. Error Estimates for Iterative Unfolding . . . . . . . . 68

B. WERM Code Listing. . . . . . . . . . . . . . 74

1. Printout of Program WERM ........................ 76

2. Printout of Subroutine MATFORM .......... 82

3. Printout of Subroutine PRINTS(NUM) . . . . . . . 84

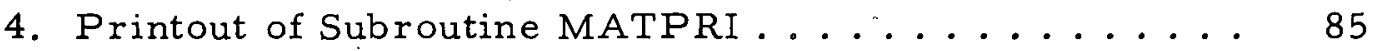

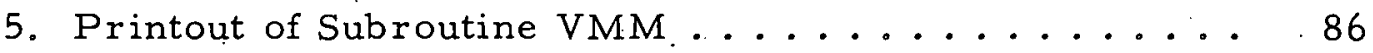

6. Printout of Subroutine READY ............ 87

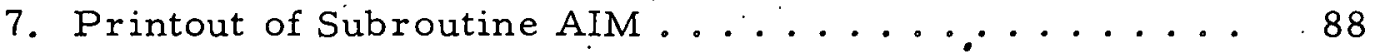

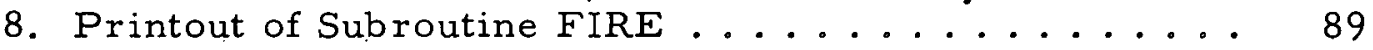

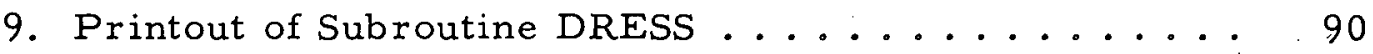

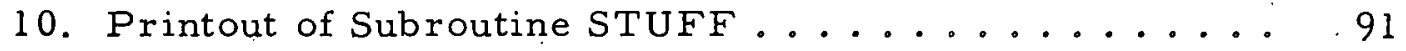

11. Printout of Subroutine MATMPY $(M, N, H, G, S) \ldots . . .91$

12. Printout of Subroutine $\mathrm{FCN}(\mathrm{M}, \mathrm{G}, \mathrm{F}, \mathrm{B}, \mathrm{Ml}) \ldots \ldots 92$

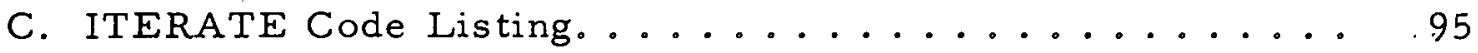

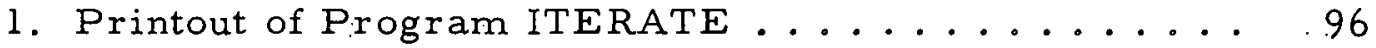

2. Printout of Subroutine MATSYM............ 98

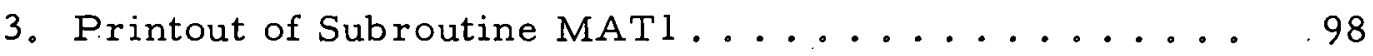

4: Printout of Subroutine MPRINT(X,N). . . . . . . . . . . . . . . . . . .

5. Printout of Subroutine MAT2........... 100

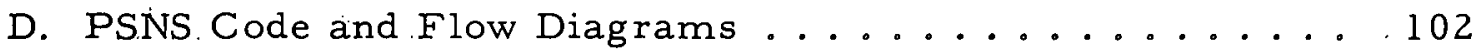

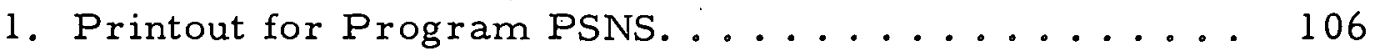

2. Printout for Function PLEG(N,ARG) ............. 111

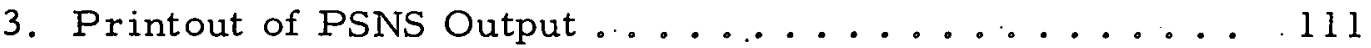

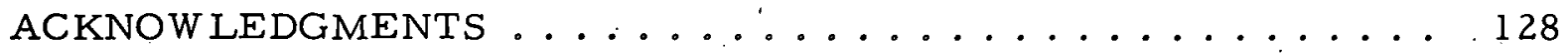

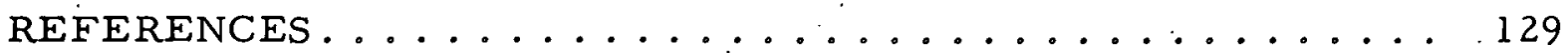




\section{LIST OF FIGURES}

No.

Title

Page

1. An Example of Spectral Distortion due to Finite-size Effects in Proton-recoil Proportional Counters ..............

2. Geometrical Variables. Affecting Proportional-counter

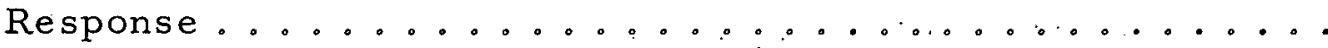

3. Pulse-height Spectrum of $\AA^{37}$ from an. Extremely Long Cylindrical Proportional Counter a n............... 14

4. Pulse-height Spectrum $A^{37}$ from a Counter with a Short

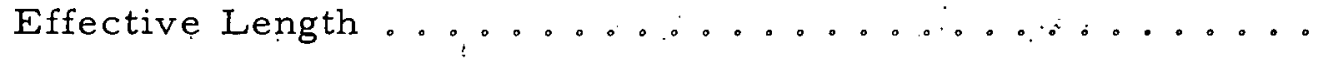

5. Relative Non-Gaussian Area versus Field-tube Diameter for Fixed Effective Length of $3.18 \mathrm{~cm}$ and Cathode Diameter of

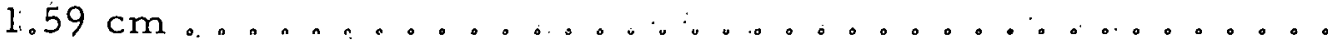

6. Relative Non-Gaussian Area versus Effective Length for Fixed Field-tube Diameter of $0.056 \mathrm{~cm}$ and Cathode Diameter of $1.59 \mathrm{~cm} \ldots \ldots \ldots \ldots \ldots \ldots$

7. Geometric Characteristics of the $4 \pi$-hydrogen-recoil

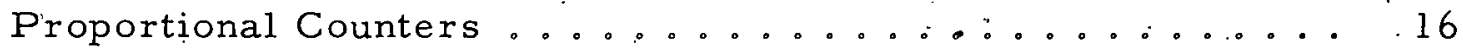

8. The Thermal-neutron Exposure Facility in ATSR $\therefore \ldots 17$

9. Response Distributions of Counter I at Total.Pressure of 81.4 psi for Nitrogen Admixtures of 10 and $50 \%$........

10. Response Distributions of Counter II at Total Pressure of 5.6. psi for Nitrogen Admixtures of 20 and $50 \% \ldots \ldots$

11. Ratio of Molecular Stopping Cross Sections in Nitrogen and Methane as a Function of Proton Energy . . . . . . . .

12. Normalized. Counter I Response Functions Corresponding to $E_{0}^{\prime}=2.26,1.99,1.63$, and $1.33 \mathrm{MeV}$ Obtained from Measure-

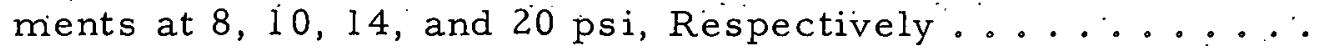

13. Normalized Counter II Response Functions Corresponding to $E_{0}^{\prime}=2.87,2.38$, and 2.1 l MeV Obtained from Measurements

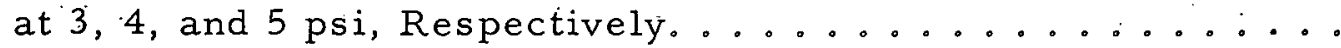

14. The Multiplicative Transformation Factor $N \cdot \epsilon\left(E_{0}-E\right) / N^{\prime} \cdot \epsilon\left(E_{0}^{\prime}-E^{1}\right) \ldots \ldots \ldots \ldots$

15. Fourier-series Approximations of the Distorted Response Function $\mathrm{D}\left(\mathrm{E}^{\prime}, \mathrm{E}_{0}^{\prime}\right)$ for Counter $\mathrm{I}$ at $\mathrm{E}_{0}^{\prime}=0.626 \mathrm{MeV} \ldots \ldots$

16. Fourier-series Apṕroximations of the Distorted Response Function $D\left(E^{\prime}, E_{0}^{\prime}\right)$ for Counter $I$ at $E_{0}^{\prime}=3: 00 \mathrm{MeV} \ldots \ldots \ldots$ 


\section{LIST OF· FIGURES}

No.

17. Fourth-order Least-squares Polynomial Approximation of Relative Gaussian Area, $G_{a}\left(E_{0}^{\prime}\right)$, for Counter $I \ldots . . . . .31$

18. Fourth-order Least-squares Polynomial Approximation of Fourier Coefficient $a_{3}\left(E_{0}^{\prime}\right)$ for Counter $I \ldots \ldots \ldots \ldots \ldots$

19. Fourth-order Least-squares Polynomial Approximation of Fourier Coefficient $a_{5}\left(E_{0}^{\prime}\right)$ for Counter I . . . . . . . . . .

20. Interpolated (Renormalized) Response Function $R\left(E^{\prime}, E_{\mathfrak{j}}\right)$ Obtained from Parametrization Process for Counter I at Midbin Energy $E_{i}=0.488 \mathrm{MeV} \ldots \ldots \ldots \ldots \ldots$

21. Interpolated (Renormalized) Response Function $R\left(E^{\prime}, E_{i}\right)$ Obtained from Parametrization Process for Counter I at Midbin Energy $E_{i}=2.44 \mathrm{MeV} \ldots \ldots \ldots \ldots \ldots$

22. $1 / E$ Test $\underline{X}$ Vector and $\underline{W}$ Vector for Counter I Delta-type Response Matrix of Orde $\bar{r}=102$ with Mesh Size of $A=19.53 \mathrm{keV}$ per Channel $\ldots \ldots \ldots \ldots \ldots \ldots \ldots$

23. Norm of Residual Vector $\mathrm{N}\left[\mathrm{R}^{(\mathrm{m})}\right]$ as a Function of Interation Number $m$ for the $1 / E$ Test Spectrum and Counter I Response Matrix ....................

24. Infinite-medium Proton-recoil Spectra Obtained from Same $\underline{W}$ Vector for Delta-type Response Matrix .............

25. Infinite-medium Proton-recoil Spectra Obtained from Same $\underline{W}$ Vector for Triangular-type Response Matrix . .........

26. Comparison of Measured Proton-recoil Spectra from Counter Counter $\mathrm{I}_{\mathrm{a}}$ (Aluminum Body, 50'-psi Methane Filling) and Counter $I_{S}$ (Stainless Steel Body, 80-psi Methane Filling) . . . .

27. Comparis on of Unfolded Infinite-medium Proton Spectra Obtained from Counters $I_{a}$ and $I_{s} \ldots \ldots \ldots \ldots$

28. Comparison of Neutron Spectra Using Iterative and Integral Unfolding. . . . . . . . . . . . . . . .

29. Block Diagram of Logical Relationship among Computer Programs. WERM, ITERATE, and PSNS ..........

30. Flow Chart A for WERM Code, Showing Treatment of Pressure-dependent Response Data. .............

31. Flow Chart B for WERM Code, Showing Computations Leading to Parametrization of $\mathrm{G}_{\mathrm{a}}\left(\mathrm{E}_{0}^{\prime}\right)$ and Fourier Coefficients

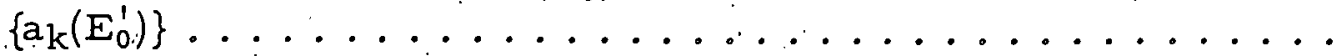




\section{LIST OF FIGURES}

No. Title

Page

32. Flow Chart C (MATFORM Subroutine) for WERM Code, Showing Formation of Matrix Elements ........... . 51

33. Flow Chart for Program ITERATE ............. 58

34. Effect of Retention of Legendre Polynomial Terms on Test

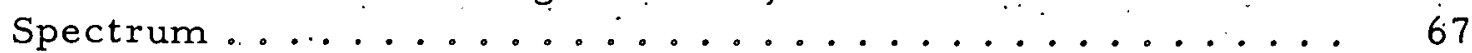

3b. Cieneral Flow Chart for PSNS Code.............. 103

36. PSNS Flow Chart $\mathrm{A}$ for Response-function Integration . . . . 104

37. PSNS Flow Chart B for Slope-forming Routine . . . . . . . 105 


\section{LIST OF TABLES}

No:

Title

Page

I. Sequence of Pressure Measuremen'ts for Counter I. . . . . 25

II. Sequence of Pressure Measurements for Counter II. . . . . . 26

III. Output of Gaussian Fitting Routine (VMM Data). . . . . . . 52

IV. Summary of Gaussian Data for Pressure Sequence . . . . . 53

V. Summary of Gaussian Parameters for Pressure Sequence . . 53

VI. Least-squares Polynomial Coefficients for Fourier Coefficients and Gaussian Area ............. 54

VII. Printout of Upper Corner of Delta Response Matrix for

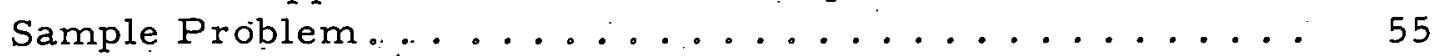

VIII. Printout of Lower Corner of Delta Response Matrix for Sample Problem ........................ 56

IX. Input Data for Iterative Unfolding of Sample Problem. . . . . . 59

X. Iterative Output for Iterations 2 and $10 \ldots 60$

XI. Raw Data for First Set of PSNS Test Problem . . . . . . . 65

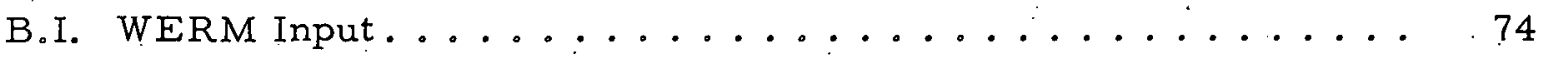

B.II. Other Variables Used in. WERM . . . . . . . . . . 75

B.III. WERM Sample Input. . . . . . . . . . . . . . . 92

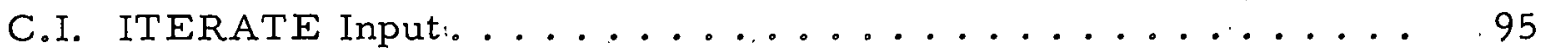

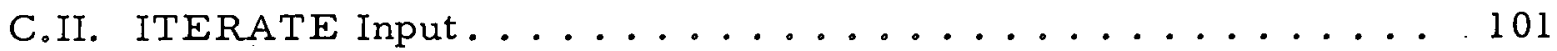

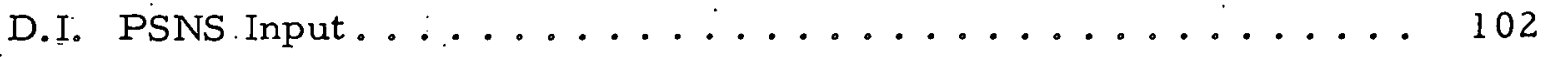

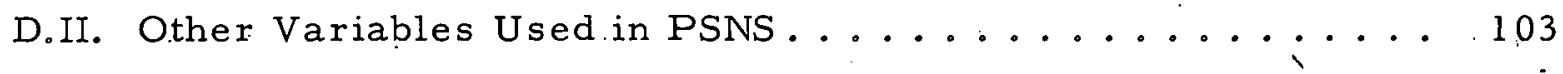

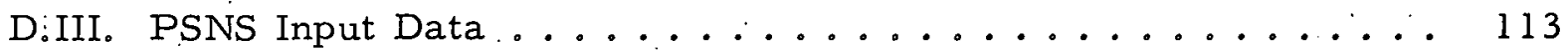

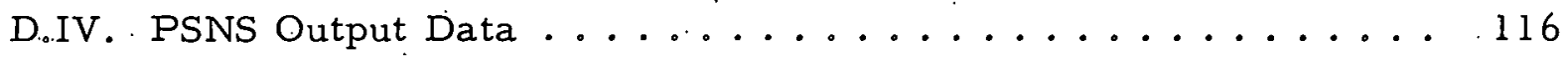




\title{
ANALYSIS AND REDUCTION OF PROTON-RECOIL DATA
}

\author{
by
}

E. F. Bennett, R. Gold, and I. K. Olson

\section{ABSTRACंT}

A process for reducing $4 \pi$ proton-recoil proportionalcounter pulse-height distributions to fast-neutron energy spectra is described. Required corrections, including those associated with finite detector size, are analyzed in detail. This procedure provides corrected neutron spectra in the region from about $1 \mathrm{keV}$ up to several $\mathrm{MeV}$. The computer programs used in this treatment (WERM, ITERATE, and PSNS) are discussed, and a sample problem illustrates and explains the manner in which these codes function.

\section{PREFACE}

This report describes a treatment of experimental data obtained in fast-neutron spectral measurements by $4 \pi$-hydrogen-recoil proportional counting. It is instructive to indicate, at the outset, the extent of the correction that arises in a neutron spectrum due to the

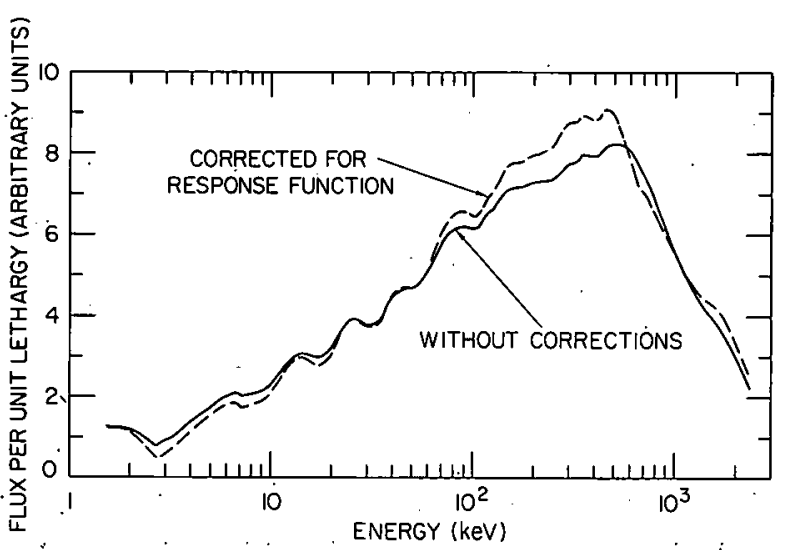

112-8539 Rev. 1

Fig: 1. An Example of Spectral Distortion due to Finite-size Effects. in Proton-recoil Proportional Counters departure of the proportional counter response from ideal. Figure 1 is a fast-reactor spectrum measurement, ${ }^{1}$ shown both with and without response-function corrections. (A complicated response function is associated with every detector of finite size.) The data have been smoothed to eliminate statistical fluctuations so that the general nature of the correction can be more readily observed. The uncorrected spectrum departs significantly from the true spectrum; the deviation is well in excess of other experimental errors. If spectrum measurements in fast reactors are to provide useful infor-

mation concerning the quality of cross-section libraries or methods of calculation, a systematic error of the kind displayed in Fig. 'l is intolerable. 
The complete correction, over the entire energy region, requires detailed experimental measurements that must be subsequently followed by an equivalent analytical effort. To describe the entire procedure, this report has been divided into four major parts, which deal with (1) the determination of proton-recoil proportional-counter response functions, (2) the unfolding of finite-size effects, (3) the reduction of proton-recoil data to neutron-flux spectra, and (4) the computer codes that perform these tasks, respectively. In Part I, the experimental methods used to obtain proportionalcounter response functions are given and the (approximate) analytical representation of the response function is obtained. Part II uses the response function generated in Part I in a treatiment of finite-size effects with both iterative and integral untolding methods. Fàr III contains a number of detailed corrections, not involving finite-size effects, that arise in the process of reducing the corrected proton-recoil data (obtained from Part II) to neutron spectra. Part IV describes the computer codes WERM, ITERATE, and PSNS that have been developed to process raw proton data. A sample problem illustrates and explains the manner in which these codes function. The detailed FORTRAN listings of these codes are presented in Appendixes $B-D$.

\section{PROTON-RECOIL PROPORTIONAL-COUNTER RESPONSE FUNCTIONS}

\section{A. Introduction.}

The determination of fast-neutron energy distributions by measurement of proton - recoil energy spectra is well established and exploited. This method is capable, in principle, of yielding accurate neutron-energy distributions, provided the corresponding proton spectra are determined precisely. In practice, limitations arise because of the finite sensitive volume of the detector. In fact, given a detector of finite sensitive volume, one cannot, in general, measure the desired proton spectrum precisely. The desired proton spectrum is by definition simply the infinite -medium proton spectrum, i.e., that spectrum attained in the limit of infinitely large sensitive volume. Accurate neutron spectra can be derived only from infinite-medium proton-recoil spectra.

The most apparent finite-size effect is related to the high-energy limit of the domain of applicability of the proton-recoil method with a given detector. This limit can be roughly. defined as the point at which the range of a recoiling. proton becomes comparable with the dimensions of the sensitive volume. In this energy region and beyond, proton energy losses outside this finite sensitive volume can lead to a significant distortion in the measured proton-recoil spectrum. The region in which the measured and infinite-medium proton-recoil spectra begin to differ significantly can be taken to define an inherent high-energy limitation of the proton-recoil method. 
This example of finite-size effects is commonly called wall-and-end effect. Because of the important role this effect plays in defining the upper energy-response limit, it has received almost exclusive attention. Treatments of the wall-and-end-effect problem have centered upon the calculation of the probability distribution for the truncation of proton tracks in the walls and ends of the detector. These methods have used direct geometrical analysis ${ }^{2-6}$ (which usually leads to approximations), Monte Carlo techniques, ${ }^{7,8}$ or astute combinations of these computational approaches. More recently, an exact analytical treatment of this geometrical problem has been developed. ${ }^{10}$

These geometrically based formulations of the wall-and-end-effect problem comprise an approximate description of finite-size effects which shall be called the geometrical model. Although these approaches have demonstrated limited success, the large amount of emphasis and concentration on the geometrical model has tended to obscure the real nature of the problem. From a more general viewpoint, the presence of the wall-andend effect implies the existence of an unfolding problem. Specifically, given the measured proton $\times$ recoil spectrum, what is really sought is the infinitemedium proton - recoil spectrum. Moreover, calculated probability distributions (within the framework of the geometrical model) can serve only to define (or approximate) the response function or response matrix of the detection system.11

The treatment of the wall-and-end-effect problem in $4 \pi$ hydrogenrecoil proportional counters used here has been formulated in this perspective. In this method, a wall-and-end-effect response matrix is determined from experimental measurements with the given recoil detector of interest. Several important advantages of this empirical formulation will become apparent in sequel. Perhaps the most crucial of these advantages lies in a more complete determination of the response matrix. Although it can be argued that the present experimental determinations may complement certain limited theoretical calculations (of the geometrical model), these measurements actually go much further. Indeed, these measurements contain a complete description of the spectral distortion, whereas the geometrical model cannot account for other sources of spectral distortion which can and often do arise. Often the basis for these additional sources of distortion may be obscure or unknown. The strength of the present empirical approach lies in the fact that these effects need not be calculated. Since all possible sources of distortion are present in the experimental. measurements, these effects are automatically included in the determination of the response matrix.

"Tip effect" is perhaps the most important example of finite-size effects not included in the geometrical model. The existence of tip effect in all proportional counters can be traced, primarily, to the presence of inhomogeneities in the electric field strength at the extremities of the 
counter. ${ }^{2,12}$ These inhomogeneities lead to nonuniform gas gain and therefore spectral distortion, which can become serious for smaller proportional counters. Consequently, for numerous applications that require proportional counters of small or miniature size, a comprehensive treatment must include a consideration of tip effect. For example, measurement of fast-reactor neutron spectra with in-core proportional-counter probes requires small sensitive volumes to keep the total event rate (signal plus background) within the processing rate capability of the electronic instrumentation. ${ }^{13,}$ i4 In such cases, tip effect can introduce a significant low-energy distortion.

The present method, which is based on experimentally determined response tunctions, divides quile ridurally intu two logical domainc. For sufficiently low-energy events (i.e., ionization events of negligible range), the response function will be energy-independent. To obtain the energyindependent proportional-counter response function, we can introduce an appropriate soft ionizing radiative decay into the counter. The present measurements have used the $A^{37}$ decay $(2.82 \mathrm{keV})$ for this purpose. The origin of any spectral distortion that arises in counting this source must. be due to tip effect.

In the second logical domain, the response function is energydependent. The energyodependent behavior of the response function in this region can be determined from a sequence of measurements using the slowneutron-induced $\mathrm{N}^{14}(\mathrm{n}, \mathrm{p}) \mathrm{C}^{14}$ reaction. From the viewpoint of this domain, the low-energy $A^{37}$-determined response function assumes an added significance. Namely, this measurement provides a low-energy boundary condition which must be satisfied by the energy-dependent response function.

Section $B$ below considers the energy-independent response function obtained from the $A^{37}$ experiments. Sections $C$ and $D$ discuss the experimental method used to obtain the energy-dependent response matrix. These sections describe the experimental measurements, the subsequent treatment of the data to determine response functions, the parametrization of the response function, and the construction of the response matrix.

\section{B. Energy-independent Response-function Measurements}

Proportional counters used for neutron spectroscopy have welldefined walls but poorly defined ends. ${ }^{2,12,15}$ At least three methods are in current use to provide an "effective end" (two ends per counter). The first method involves a field tube of small diameter relative to the cathode and aligned concentrically with the anode wire. The guard field tube is kept at a potential appropriate to the anode field and field lines terminate on the guard tube in the region near the ends, thereby preventing the collection of ionization that originates outside the guard tube. A simpler (and consequently more widely used) method is to increase the diameter of the anode wire in the end region. Gas multiplication depends very sensitively upon anode 
diameter, and an increase by several times in the anode diameter will decrease multiplication by several orders of magnitude. This method was used exclusively for measurements described in this report and is especially well suited for counting tubes of small size. The third method, applicable to counters having spherical cathodes, ${ }^{15}$ uses careful placement of electrical insulation near ends. The tendency for the electric field to increase with decreasing cathode diameter is counteracted by the presence of the insulation.

In practice, and especially as the effective length of counters. decreases, the response function will depart from ideal. It is not possible, even with the first method outlined above, to produce an ideal response, since lateral diffusion of fonization from proton recoils will occur and events near ends may be truncated regardless of the method of field shaping.

Figure 2 shows the parameters that can be varied in designing a cylindrical counter desensitized by an anode-diameter increase near the

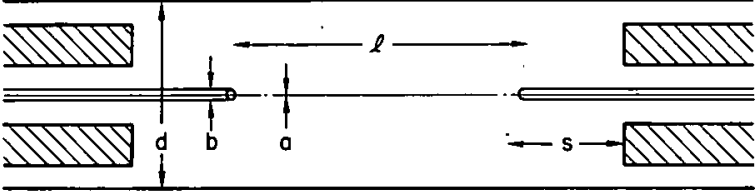

$112-8868$

Fig. 2. Geometrical Variables Affecting Proportionalcounter Response ends. That a reduction in counter size (of $d$ and/or $\ell$ ) to minimal values may be beneficial from an experimental viewpoint is clear from the following considerations. Sensitivity of counters to gamma background is nearly proportional to the total wall area, since most conversion electrons originate through interactions in the walls. The number of hydrogen

atoms in the effective counting region will determine the rate of proton recoils. Ordinarily resolution can be maintained in counters if gas pressure is increased in proportion to any reduction in diameter. Wall-and-end-effect distortion (which is governed by the ratio of gas pressure to counter diameter) will remain essentially unchanged if gas pressure is increased in proportion to any reduction in counter size. The upshot is that a reduction in counter size, accompanied by a corresponding increase in gas pressure, will improve the signal (proton-recoil)-to-background (gamma) rate while maintaining wall-and-end-effect problems (as well as detector resolution) fixed. The neutron sensitivity of the detector will also decrease, and this is desirable for experiments with a high neutron background (due to spontaneous fission) such as will be found in plutonium-fueled fast reactors. Where a high neutron sensitivity is desirable, counter size can be increased with an attendant improvement in internal electric field properties and a more nearly ideal response function.

The obvious way to test for response-function effects of the kind mentioned is to observe counter pulse-height spectra in response to monoionizing events; these events must be induced uniformly over the counter and have negligible track extension. The electron emitted by the decay of $\mathrm{A}^{37}$ ( $\left.2.8 \mathrm{keV}\right)$ by $\mathrm{K}$ capture is a convenient test source; it is inexpensive and can be added in small amounts to the normal filling gas. 
Figure 3 is an $\mathrm{A}^{37}$ pulse-height spectrum obtained in an extremely long cylindrical proportional counter designed for cosmic-ray experiments. ${ }^{16}$

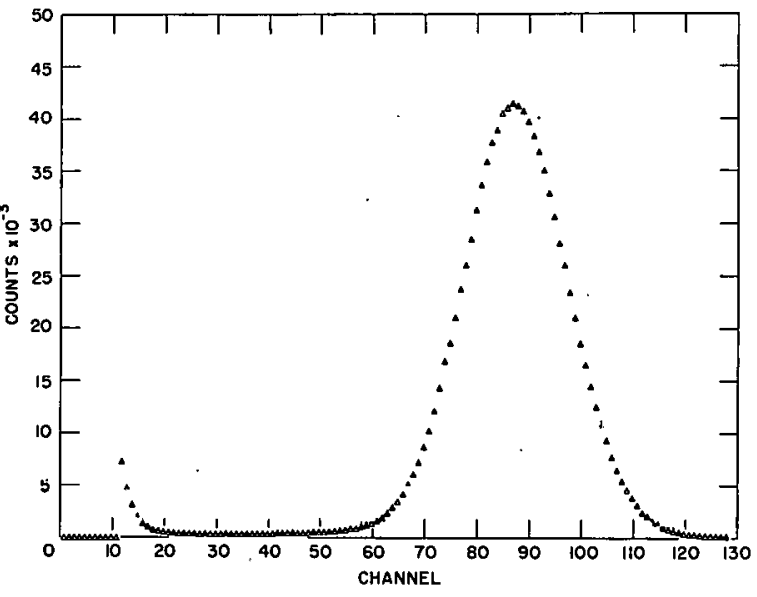

$112-8919$

Fig. 3. Pulse-height Spectrum of $A^{37}$ from an Extremely Long Cylindrical Proportional Counter

Contrast this result with Fig. 4, which displays the $\mathrm{A}^{37}$ measurement for a much smaller counter $(l=5.0 \cdot \mathrm{cm}$, $\mathrm{d}=1.59 \mathrm{~cm})$. Distortion effects are quite apparent in the smaller counter and indicate that the electric field near the ends does not change as abruptly as would be desired due to the excessive thickening of the anode near the ends even for $b=0.0254 \mathrm{~cm}$. (Some lateral diffision of electrurs may also be contributing, although the two effects cannot be distinguished.) A similar distribution was observed for the smaller counter at widely differing gas pressures and with different gases, indicating that the distribution is of esentially

"mechanical" origin and is little influenced by gas type or multiplication. At low channels, electrons from the L-shell decay of $\mathrm{A}^{37}$ appear and cause the distributions to increase just above discriminator cutoff. In the analysis of distributions from $A^{37}$ for response-function information, the L-s hell component has been removed, and in effect distributions have been extrapolated linearly into the lowlying channels.

To examine more closely the influence of the counter parameters depicted in Fig. 2 upon the response function, we measured the $\mathrm{A}^{37}$ spectrum using a variety of counters. Various combinations of $\mathrm{d}, \mathrm{b}$, and $\ell$ (the anode a was fixed at $0.0254 \mathrm{~mm}$ ) were tested, and in each case the

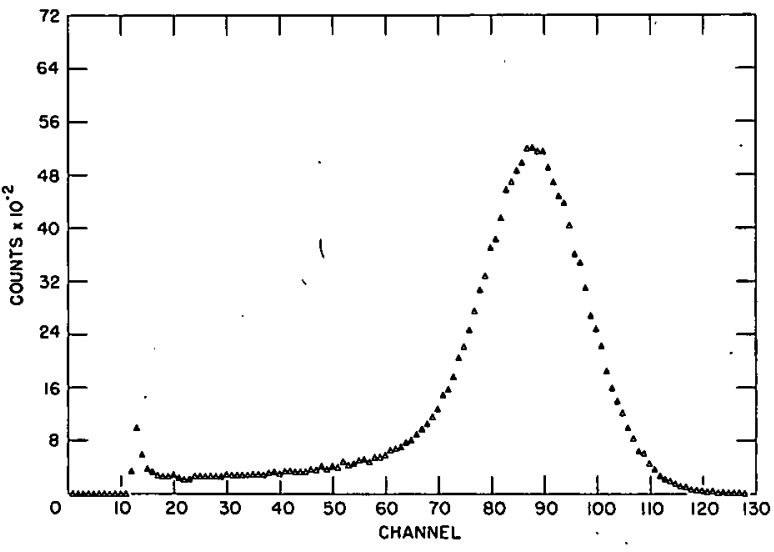

$112-8918$

Fig. 4. Pulse-height Spectrum of $A^{37}$ from a Counter with a Short Effective Leng th pulse-height distribution resembled that of Fig. 4 but with relatively more or less of a contribution below the main peak. Each spectrum was fitted, over the peak, by a Gaussian dis tribution, and the area included in the non-Gaussian region was compared with the total area. This ratio (the relative non-Gaussian area, $1-G_{a}$ ), which would be zero for an ideal counter (as shown in Fig. 3), varied with. counter design. Figure 5 is a plot of this ratio versus the diameter, $b$, of the field desensitizing tube for a counter having an inside diameter, $d$, of 
$1.59 \mathrm{~cm}$ and an effective length, $l$, of $3.18 \mathrm{~cm}$. We see clearly the desirability of keeping $b$ as small as possible (consistent with an adequate reduction in gas multiplication). Figure 6 presents the relative non-Gaussian area versus the effective length of a counter, $l$, having a fixed $(1.59-\mathrm{cm})$ inside diameter, d, and fixed (0.056-cm) field tube, b. As expected, the effects of distortion decrease markedly with increasing effective length.

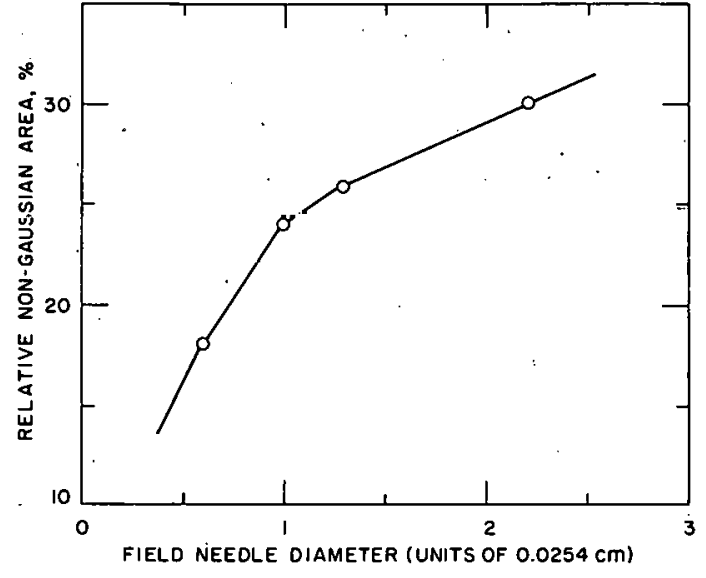

$112-8871$

Fig. 5. Relative Non-Gaussian Area versus Field-tube Diameter for Fixed Effective Length of $3.18 \mathrm{~cm}$ and Cathode Diameter of $1.59 \mathrm{~cm}$

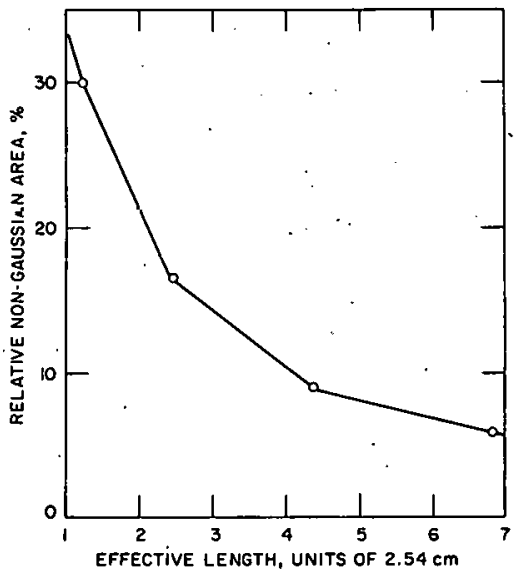

$112-9011$

Fig. 6. Relative Non-Gaussian Area versus Effective Length for Fixed Fieldtube Diameter of $0.056 \mathrm{~cm}$ and Cathode Diameter of $1.59 \mathrm{~cm}$

In the above measurements, the separation s (see Fig. 2) was maintained at about a counter cathode diameter. The distributions were not sensitive to $s$ with $s$ chosen in excess of about one cathode radius.

\section{Energy-dependent Response Function}

\section{Experimental Measurements}

The basis of the present method lies in the determination of the response function of a given proportional counter to monoenergetic protons. To this end, we studied a sequence of measurements containing various total gas pressures in a single counter, all of which included an appropriate amount of nitrogen. The response distribution for each pressure was obtained from an exposure of the counter to a thermalneutron flux. In such an environment, monoenergetic protons from the $\mathrm{N}^{14}(\mathrm{n}, \mathrm{p}) \mathrm{C}^{14}$ reaction arise throughout the entire volume of the counter. In this manner, a sequence of experimental response distributions is obtained, one for each total counter gas pressure used. The transformation of this sequence into a sequence of energy-dependent proton-response functions in a counter of fixed pressure is discussed in Section 2 below.

The present series of experiments were conducted using the rmal neutrons in the graphite reflector of the Argonne Thermal Source Reactor (ATSR). ${ }^{17}$ For these measurements, two proportional counters were 
constructed of aluminum bodies. Figure 7 shows the geometric characteristics of these counters. The smaller counter shown in Fig. 7a (Counter I) is an exact duplicate of a stainless steel proportional counter used for incore fast-neutron spectra experiments in a plutonium-fueled critical assembly. Aside from a shorter length, Counter II, as shown in Fig. 7b, is otherwise geometrically identical to a one-meter-long stainless steel proportional counter used in cosmic-ray neutron experiments. ${ }^{16}$ The length of Counter II is a compromise between the practical size limitations of the thermal-neutron exposure facilities and the desire to maintain a good approximation of the length-to-diameter ratio of the cosmic-ray neutron counter. Figure 8 shows the location and arrangement of Counters I and II in the ATSR graphite reflector.

The quality of thermal flux uscd in these experiments is important since precise pulse-height distributions are required for an adequate definition of the response matrix. In particular, the presence of an appreciable component of fast flux could result in an undesirable contribution of proton recoils in the measured pulse-height distributions. To guard against such a possibility, the counters were located in a position (as shown in Fig. 8) that possessed a cadmium ratio larger than 2000.* In addition, measurements were made with methane fillings only. The complete absence of nitrogen permits a direct examination of the background pulseheight distribution. Preliminary background distributions were obtained in this manner with stainless steel proportional counters. This investigation revealed significant background pulse-height distributions induced by thermal-neutron reactions in the stainless steel counter walls. To solve this problem for the proportional counters of interest, aluminum counters possessing identical geometric characteristics were constructed, as described above. In this manner, thermal-neutron-induced wall reactions and capture-gamma background were considerably reduced. Fast-reactor spectrum measurements ${ }^{1}$ have normally been made using stainless steel counters, and any reactions involving counter walls have been observed to

(o) COUNTER I

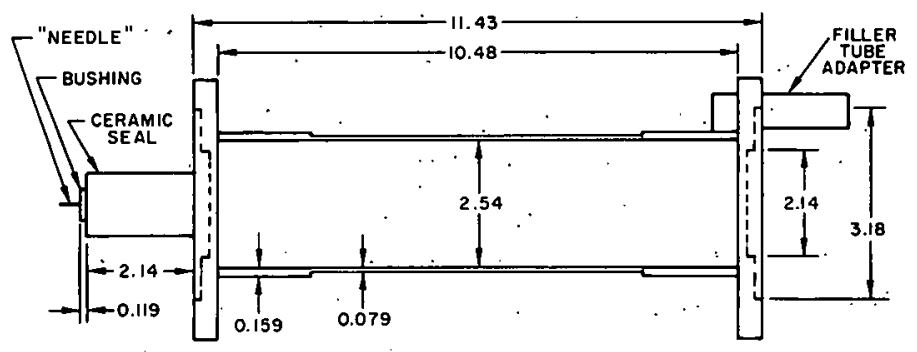

ALL DIMENSIONS IN CENTIMETERS

$112-9038-\mathrm{A}$

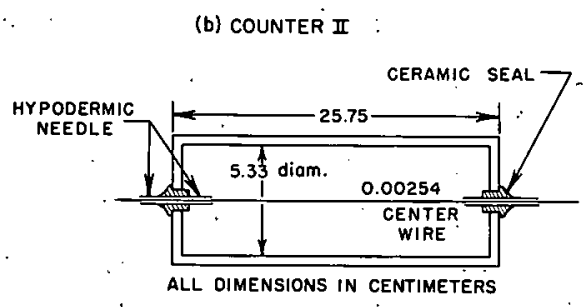

112-9038-B Rev. 1

Fig. 7.- Geometric Characteristics of the $4 \pi$-hydrogen-recoil Proportional Counters

* This cadmium ratio was measured with bare and cadmium-covered gold foils and is defined as the ratio of bare-to-cadmium-covered saturated activity. 


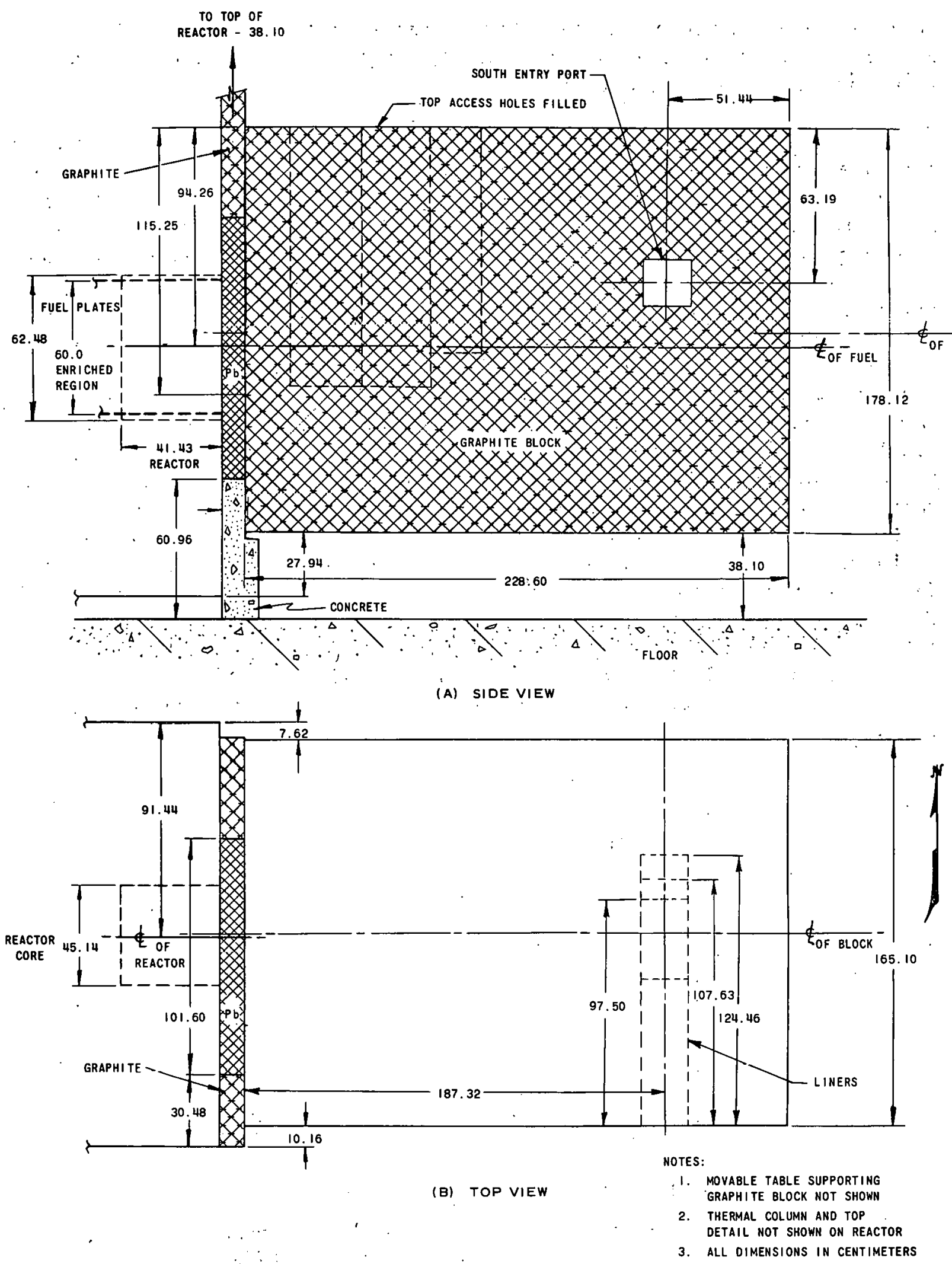

112-6638 Rev. 1

Fig. 8. The Thermal-neutron Exposure Facility in ATSR 
be at lease three orders of magnitude below normal proton-recoil rates: For the response-function tests in highly thermal fluxes, however, an aluminum body was preferable.

In the energy-dependent response region, proton-recoil proportional counters are filled with methane. Consequently, it is desirable to include only a small relative percentage admixture of nitrogen in the pressure-dependent sequence of methane fillings. On the other hand, the intensity of the desired pulse-height distribution is controlled by the size of this nitrogen admixture. Under conditions of a few percent nitrogen admixture, background distributions could not be considered negligible at the low end of the required pressure sequence of measurements, in spite of the background reductions attained with the aluminum counters. Further investigation revealed, quite fortunately, that the experimental response was independent of the percentage nitrogen admixture within the experimental error of the individual response-function measurements. This behavior is demonstrated for Counters I and II, in Figs. 9 and 10, respectively. Figure 9 contains the response distributions obtained from Counter I at a total pressure of 81.4 psi for relative nitrogen admixtures of 10 and $50 \%$. Figure 10 shows the response distributions of Counter II for a total pressure of 5.6 psi with relative nitrogen admixtures of 20 and $50 \%$. These distributions are normalized to equal area. The only apparent differences that can be observed correspond to slight changes in the resolution attained. Such minor differences must be anticipated, since small changes in resolution are common and accompany successive counter fillings which maintain constant gas mixtures.

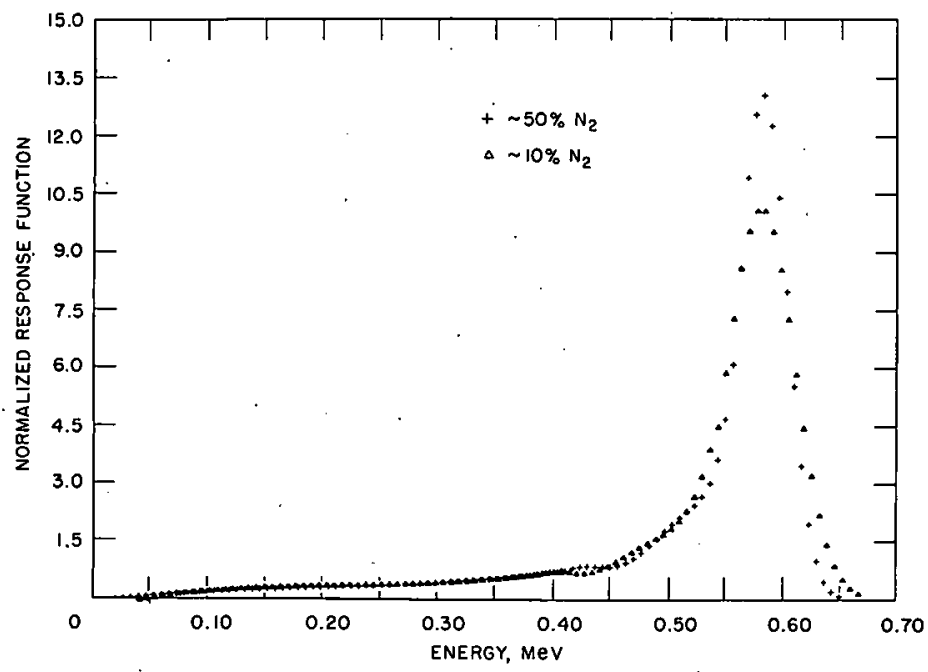

112-8936 Rev. 1

Fig. 9. Response Distributions of Counter I at Total Pressure of 81.4 psi for Nitrogen Admixtures of 10 and $50 \%$ 


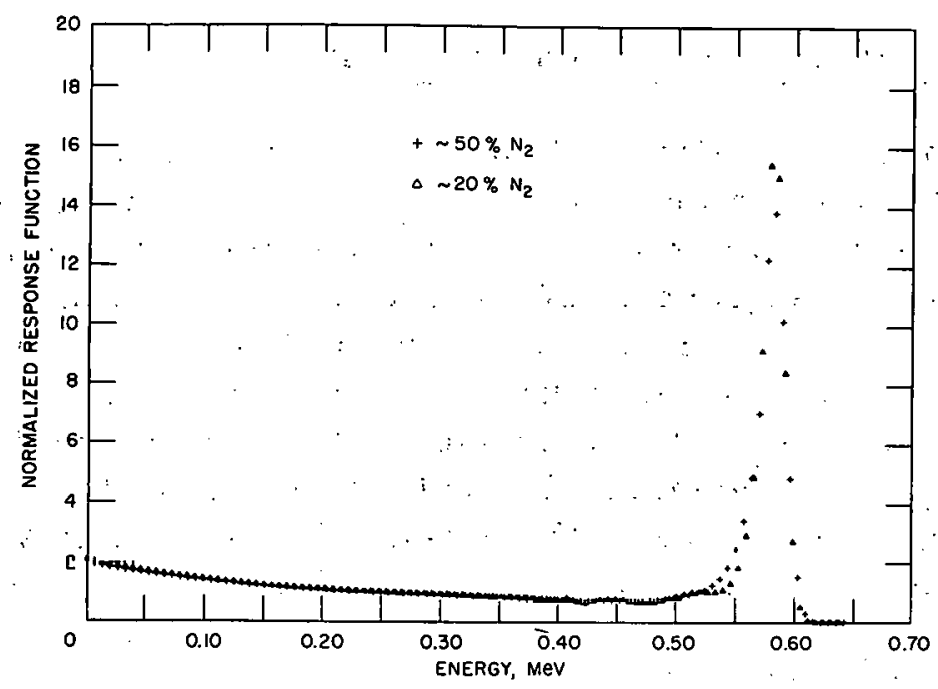

$112-9021$

Fig. 10. Response Distributions of Counter II at Total Pressure of 5.6 psi for Nitrogen Admixtures of 20 and $50 \%$

These results, obtained with geometrically different counters and at different pressures, imply that the experimental measurements are generally independent of the percentage nitrogen admixture. This conclusion can be traced to the behavior of the molecular stopping cross section, $\epsilon(E)$, for protons in methane and nitrogen. Figure 11 shows that the stopping cross sections for these two gases are not significantly different below $700 \mathrm{keV}$. Here the ratio $\epsilon_{\mathrm{N}_{2}} / \epsilon_{\mathrm{CH}_{4}}$, obtained from the data of Whaling, ${ }^{18}$ is plotted as a function of proton energy. It can be seen that this ratio does not significantly deviate from unity. We can therefore use any convenient nitrogen admixture and still obtain a response function representative of methane. The present sequence of pressure measurements used nitrogen admixtures of approximately $50 \%$. Under these conditions, background contributions were generally negligible.

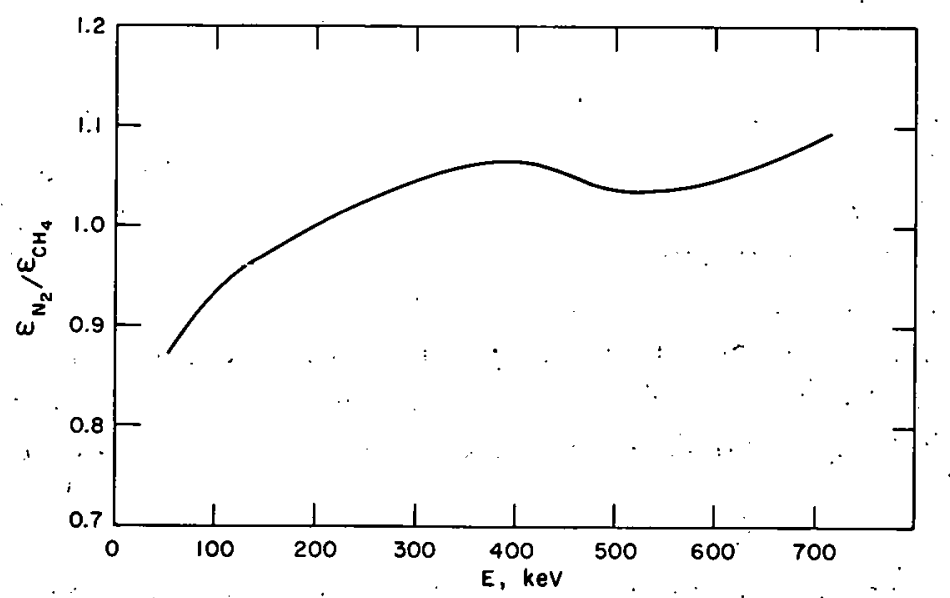

112-8870 Rev. 1

Fig. 11. Ratio of Molecular Stopping Cross Sections in Nitrogen. and Methane as a Function of Proton Energy 


\section{Energy-density Transformations}

Consider a uniform-isotropic distribution of monoenergetic protons in a $4 \pi$-recoil proportional counter. It will become evident, in sequel, that the sensitive volume of the counter can be of arbitrary geometry. Let $\mathrm{N}$ be the molecular density of the counter gas, and $E_{0}$ the energy of the monoenergetic protons. Under such circumstances, we can introduce the track-length probability distribution of proton events $P(l ; r)$, where $l$ is the track length and $r=r\left(E_{0}\right)$ is the range of the monoenergetic protons in the counter gas. Given monoenergetic protons of energy $E_{0}, P(\ell, r) d \ell$ is the probability of proton-track lengths arising between $\ell$ and $\ell+\mathrm{d} l$ in the sensitive volume of the detector.

The relation between the actual response function of the counter to monoenergetic protons of energy $E_{0}, R\left(E_{,} E_{0}\right)$, and this track-length probability distribution is (neglecting resolution broadening)

$$
R\left(E, E_{0}\right) d E=R\left[E(\ell), E_{0}(r)\right] \frac{d E}{d \ell} d \ell
$$

hence,

$$
P(\ell, r)=R\left(E, E_{0}\right) \frac{d E}{d \ell}
$$

Equations la and $\mathrm{lb}$ imply the existence of a continuous function, $\ell\left(E, E_{0}\right)$, relating proton track length and proton energy loss, $E$, in the sensitive volume of the detector. This fundamental relation can be written in the form ${ }^{18}$.

$$
\ell\left(E, E_{0}\right)=\frac{1}{N} \int_{E_{0}-E}^{E_{0}} \frac{d \xi}{\epsilon(\xi)},
$$

where $\epsilon(\xi)$ denotes, as before, the molecular stopping cross section. Equations 1 and 2 apply strictly only to events originating in the sensitive volume and escaping with track length $\ell$. Some events originate in the region near the ends and may enter the effective volume and terminate there or continue on into a wall or into the opposite end. These events will all appear in the experimentally observed distributions. They will be few in number, relative to the totality of events, and it is not necessary to include this refinement in the treatment of relative stopping power given here.

According to the Bethe-Livingston-Bloch formula, ${ }^{19}$

$$
\epsilon(\xi)=C_{1} \frac{Z}{\xi}\left(\ln \frac{\xi}{Z}+C_{2}\right)
$$


where $Z$ is the atomic number of the medium and $C_{1}$ and $C_{2}$ are constants. For protons,

$$
C_{1}=2 \pi e^{4} \frac{m_{p}}{m_{e}}
$$

and

$$
\mathrm{C}_{2}=\ln \left(\frac{4}{\mathrm{I}_{0}} \cdot \frac{\mathrm{m}_{\mathrm{e}}}{\mathrm{m}_{\mathrm{p}}}\right)
$$

whicie $\mathrm{e}$ is the electron charge, $\mathrm{me}_{\mathrm{e}}$ is the electron mass, $\mathrm{mp}_{\mathrm{p}}$ is the proton mass, and $\mathrm{I}_{0}$ is an empirical constant.

In terms of Eq. 2, the proton range is given by

$$
r\left(E_{0}\right)=\ell\left(E_{0}, E_{\prime_{0}}\right)=\frac{1}{N} \int_{0}^{E_{0}} \frac{d \xi}{\epsilon(\xi)} \text {. }
$$

It follows that the track length $\ell$ can be expressed as the difference between two ranges. We have

$$
\ell\left(E, E_{0}\right)=\frac{1}{N}\left[\int_{0}^{E_{0}} \frac{\mathrm{d} \xi}{\epsilon(\xi)}-\int_{0}^{E_{0}-E} \frac{\mathrm{d} \xi}{\epsilon(\xi)}\right]
$$

or

$$
\ell\left(E, E_{0}\right)=r\left(E_{0}\right)-r\left(E_{0}-E\right)
$$

Let us now consider simultaneous energy-density transformations $(E, N) \rightarrow\left(E^{\prime}, N^{\prime}\right)$, where $E^{\prime}$ and $N^{\prime}$ represent new energy and molecular:density variables, respectively. To obtain the response function from the pressure-dépendent sequence of $\mathrm{N}^{14}(\mathrm{n}, \mathrm{p}) \mathrm{C}^{14}$ measurements, energy-density transformations will be used that leave the track length (and therefore the track-length probability distribution) invariant. In view of Eq. 6, the requirement that $l$ be invariant can be satisfied, provided the range, $r$, is invariant under this transformation. This condition can be written in the form

$$
r^{\prime}\left(E^{\prime}\right)=r(E)
$$

or, morè specifically,

$$
\frac{1}{N !} \int_{0}^{E^{\prime}} \frac{d \xi}{\epsilon(\xi)}=\frac{1}{N} \int_{0}^{E} \frac{d \xi}{\epsilon(\xi)}
$$


Conversely, if $\mathrm{N}, \mathrm{N}^{\prime}$, and $E$ are prescribed, then Eqs. $7 \mathrm{a}$ and $7 \mathrm{~b}$ define a transformation $E \rightarrow E^{\prime}$ which leaves $l$ invariant.

Using such a transformation, we find from Eq. lb,

$$
P(\ell, r)=R\left(E, E_{0}\right) \cdot \frac{d E}{d \ell}=R\left(E^{\prime}, E_{0}^{\prime}\right) \frac{d E^{\prime}}{d \ell^{\prime}}
$$

Hence the response function due to monoenergetic protons of energy $E_{0}^{\prime}$ is given by*

$$
R\left(E^{\prime}, E_{0}^{\prime}\right)=\left(\frac{d E^{\prime}}{d \ell^{\prime}}\right)^{-1} \cdot\left(\frac{d E}{d \ell}\right) \cdot R\left(E, E_{0}\right),
$$

where the Jacobians that arise in Eq. 9 can be determined from Eq. 2 . We have**

$$
\frac{\mathrm{dE}}{\mathrm{d} \ell}=\mathrm{N}: \epsilon\left(\mathrm{E}_{0}-\mathrm{E}\right)
$$

and

$$
\frac{\mathrm{d} E^{\prime}}{\mathrm{d} \ell^{\prime}}=\mathrm{N}^{\prime} \cdot \epsilon\left(\mathrm{E}_{0}^{\prime}-\mathrm{E}^{\prime}\right)
$$

Using Eqs. 10a and 10b, Eq. 9 assumes the form

$$
R\left(E^{\prime}, E_{0}^{\prime}\right)=\frac{N \cdot \epsilon\left(E_{0}-E\right)}{N^{\prime} \cdot \epsilon\left(E_{0}^{\prime}-E^{\prime}\right)} \cdot R\left(E, E_{0}\right) \text {. }
$$

We can demonstrate that Eq. 1l, together with the energydensity transformation equations (i.e., Eqs. $7 \mathrm{a}$ and $7 \mathrm{~b}$, provide a basis for the definition of the energy-dependent response function (or response matrix). To this end, let $R\left(E, E_{0}\right)$ be the observed pulse-height distribution due to monoenergetic protons of energy $E_{0}$ in a counter possessing gas density $N$. The transformed distribution provided by $\mathrm{Eq}$. $11, \mathrm{R}\left(\mathrm{E}^{\prime}, \mathrm{E}_{0}^{\prime}\right)$, corresponds to the response. function due to monoenergetic protons of energy $E_{0}^{\prime}$ in a counter possessing a molecular gas density $\mathrm{N}^{\prime}$. Consider, therefore, a sequence of measurements with different gas densities $\left\{\mathrm{N}_{\mathrm{i}}\right\}$, $i=1,2, \ldots, n$, undertaken with a fixed value of $E_{0}$. These measurements give rise to a sequence of transformations $\left(N_{i}, E_{0}\right) \rightarrow\left(N_{i}^{\prime}, E_{0}^{\prime}\right), i=1,2, \ldots, n$. For each transformation, the measured response distribution $R\left(E, E_{0}\right)$ is . mapped into. a response function $R\left(E^{\prime}, E_{0}^{\prime}\right)$. In particular, if we choose

\footnotetext{
* The transformation properties of the response function $R\left(E, E_{0}\right)$ are therefore those of a relative scalar of weight one (or weight unity).

*** These equations assume that the Jacobians do not vanish in the region of interest; this assumption is justified on physical grounds, cf. Eqs. 10a and $10 \mathrm{~b}$.
} 
$N_{i}^{\prime} \equiv N^{\prime}, i=1,2, \ldots, n$, then all transformed response functions $\left\{R_{i}\left(E^{\prime}, E_{0}^{-1}\right)\right\}$ will correspond to a single counter gas density, namely $N^{\prime}$. The set of response functions so obtained constitute a representation of the complete ene-rgy-dependent response, including wall-and-end effects, of the $4 \pi$ hydrogen-recoil proportional counter (with molecular gas density $N^{\prime}$ ).

The empirical basis of the present method rests upon the relationship between the measured response distribution, $\mathrm{R}\left(\mathrm{E}, \mathrm{E}_{0}\right)$, and the transformed response function, $R\left(E^{\prime}, E_{0}^{\prime}\right)$. According to Eq. lil, each trans formed response function is simply obtained from a measured distribution by applying a multiplicative factor. This factor is the ratio of the two aforementioned Jacobians. If this multiplicative factor is a constant, i.e., independent of energy, then the measured and transformed response distributions coincide. In this event, the raw experimental data directly determine the complete response function. What actually occurs in practice is quite close to this case. Indeed, as will be demonstrated, the multiplicative factor is constant except in the region $\mathrm{E} \rightarrow \mathrm{E}_{0}$ (or correspondingly $\left.E^{\prime} \rightarrow E_{0}^{\prime}\right)$. Consequently, over almost the entire energy domain, we are actually using measured distributions directly. In this manner, all possible distortions that arise, such as the tip effect (see p. 11), are automatically included in the response function. Use of these empirically determined response distributions is also sufficient justification for not extending Eqs. 1 and 2 to include events entering the effective counting region from the ends. More specifically, it can be shown that the formulation provided here is applicable for all but a small portion of the se end events. In fact, the present treatment is valid for all events entering the ends that lie outside the region $\mathrm{E} \rightarrow 0$ (or correspondingly $\mathrm{E}^{\prime} \rightarrow 0$ ).

Before proceeding to the experimental measurements, we must stress that the underlying assumption of the present formulation, namely the existence of a continuous range-energy relation, rests upon a strong experimental and theoretical base. Range-energy tables for protons (in gases of interest) exist with at most 1 or $2 \%$ relative error. ${ }^{18}$ Conversely, any method that uses a given range-energy relation must possess the limitations inherent in this relation. Thus, the treatment of finite-size effects for electrons, rather than protons, is of considerably greater complexity. In contrast with the proton case, for electrons we can no longer assume that straggling is negligible. It is evident, however, that if such a lack of definition in the range-energy relation exists, it is an additional complexity not only for the present formulation but also for all other formulations.

It is now clear that the applicability of the present treatment extends to proportional counters of arbitrary geometry. However, the response matrices defined by the present thermal-neutron experiments are actually isotropic response matrices. From a rigorous viewpoint, these response matrices are directly applicable for unfolding finite-size 
effects in counters of arbitrary geometry, only when the angular neutron flux is isotropic. However, if the counter possesses symmetry, the applicability of the isotropic response matrix may be extended to cases where the flux anisotropy coincides with the counter symmetry. In this event, the counter response is equivalent to what would arise in an isotropic flux. It follows that spherical counters may be used in any flux; cylindrical counters are allowed anisotropic flux in the radial direction, but not in the axial direction. Isotropic response matrices can also be used for nonisotropic angular flux, provided the angular dependence of the flux is known. In this event, a series of measurements can be carried out for selected angular orientations of the counter in the nonisotropic flux. A proper choice of angles and/or time-integrated neutron intensity at given orientations, for this sequence of measurements, can provide dil integrated exposure equivalent to an incident isotropic neutron flux. The isotropic response matrix could then be used for unfolding the sum of this chosen sequence of measurements.

\section{Analytical Formation of the Response Function}

\section{Treatment and Parametrization of Experimental Data}

We have pointed out that the present description can be imple mented with the thermal-neutron-induced $\mathrm{N}^{14}(\mathrm{n}, \mathrm{p}) \mathrm{C}^{14}$ reaction. In this manner, however, the pure monoenergetic proton source required by this treatment is only approximately attained, since the $\mathrm{C}^{14}$ reaction product does carry off a small fraction of the available energy. To account for the contribution, the customary ionization-equivalent-energy calibration of the pulsemheight scale is modified by subtracting the ionization equivalent energy of the $\mathrm{C}^{14}$ recoil (approximately $31 \mathrm{keV}^{*}$ ) from the calibrated energy of each pulse weight channel. This small correction establishes a value of $E_{0}=584 \mathrm{keV}$ for the monoenergetic proton source used in the present series of measurements.

There exists an additional and important low-energy limitation inherent in all experimentally determined response distributions, namely that any measured distribution, such as $R\left(E, E_{0}\right)$, cannot be determined over the complete energy range $(0, \infty)$. In particular, a low-energy cutoff must be introduced to bias out both electronic noise and gamma-ray-induced events which arise in the lowest pulse-height channels. In the present measurements, $R\left(E_{,} E_{0}\right)$ has been obtained below this cutoff by a smooth extrapolation of the measured pulse-height data in the channels above this cutoff.

* The ionization equivalent $C^{14}$ recoil energy was determined from a comparison of the $N^{14}(n, p) C^{14}$ reaction Q-value and experimentally observed proportional-counter response to fast monoenergetic accelerator neutrons. 
This treatment is not arbitrary and can be justified in terms of the foregoing analysis. "Using Eqs. 8 and 10a, we can write

$$
\lim _{E \rightarrow 0}\left[R\left(E, E_{0}\right) \cdot N \in\left(E_{0}-E\right)\right]=\lim _{\ell \rightarrow 0} P(\ell, r)
$$

or

$$
\lim _{E \rightarrow 0} R\left(E, E_{0}\right)=\frac{\lim _{\ell \rightarrow 0} P(\ell, r)}{N \cdot \lim _{E \rightarrow 0} \epsilon\left(E_{0}-E\right)} .
$$

It follows from Eq. $12 \mathrm{~b}$ that for small $\mathrm{E}, \mathrm{R}\left(\mathrm{E}, \mathrm{E}_{0}\right)$ will be a slowly varying function. It can be generally established that $P(\ell, r)$ is constant for $\ell \ll r$. Moreover, $\epsilon(\xi)$ is a smooth and slowly varying function for $\xi$ in the neighborhood of $E_{0}$. Consequently, smooth extrapolation of the experimental data into the region below the low-energy cutoff is justifiable.

Tables I and II summarize the sequence of pressure measurements carried out with Counters I and II, respectively. The values of $E_{0}^{\prime}$ listed in these tables correspond to methane operating pressures of $80 \mathrm{psi}$

TABLE I. Sequence of Pressure Measurements for Counter I .

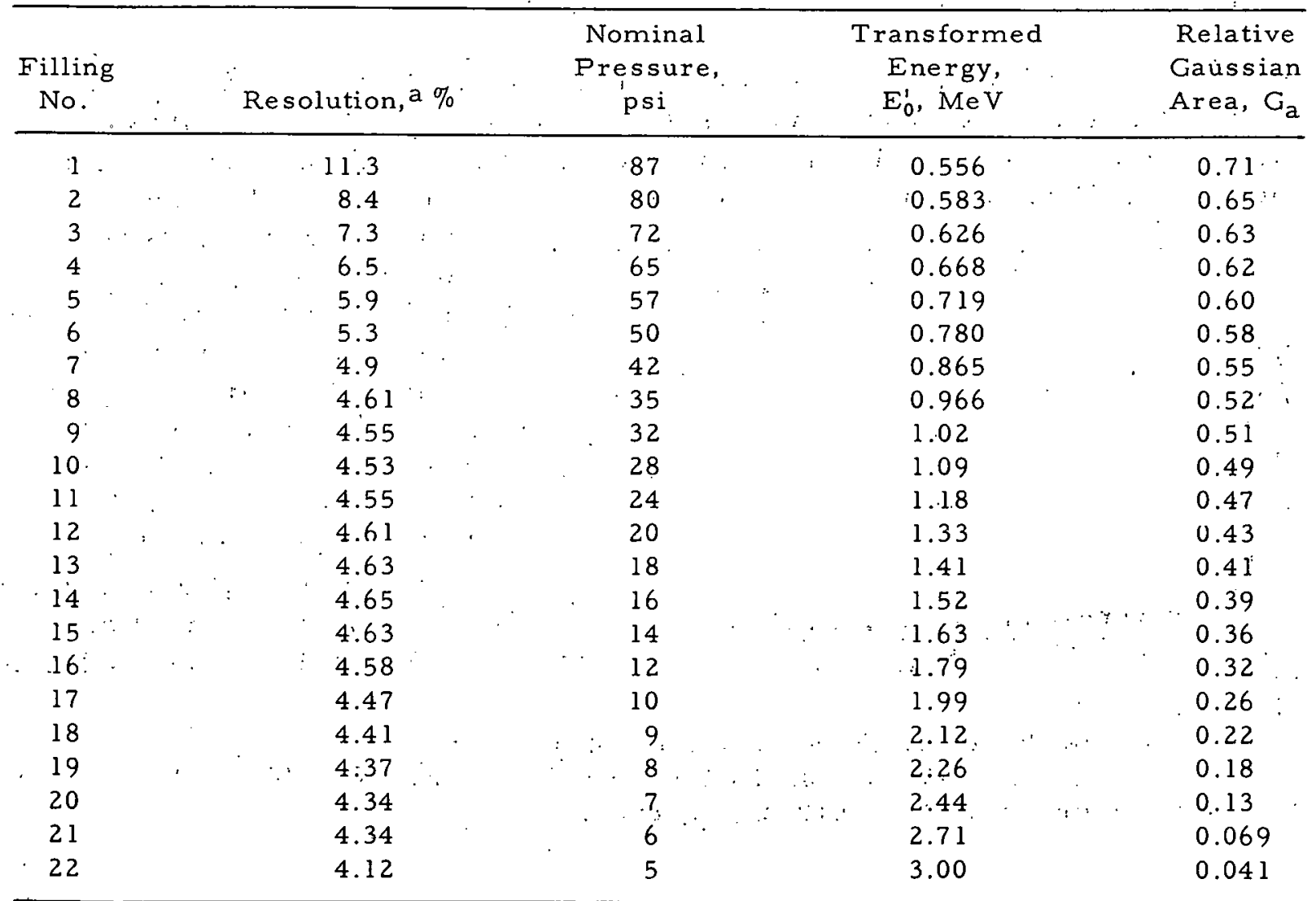

abserved relative full width half maximum (FWHM). 
TABLE II. Sequence of Pressure Measurements for Counter II

\begin{tabular}{ccccc}
\hline $\begin{array}{c}\text { Filling } \\
\text { No. }\end{array}$ & Resolution, $\begin{array}{c}\text { a } \% \\
\begin{array}{c}\text { Nominal } \\
\text { Pressure } \\
\text { psi }\end{array}\end{array}$ & $\begin{array}{c}\text { Transformed } \\
\text { Energy, } \\
E_{0}^{\prime}, \mathrm{MeV}\end{array}$ & $\begin{array}{c}\text { Relative } \\
\text { Gaussian } \\
\text { Area, G }\end{array}$ \\
\hline 1 & 8.9 & 45 & 0.583 & 0.946 \\
2 & 8.1 & 40 & 0.626 & 0.920 \\
3 & 7.7 & 30 & 0.657 & 0.90 \\
4 & 7.0 & 33 & 0.708 & 0.87 \\
5 & 6.6 & 30 & 0.749 & 0.85 \\
6 & 6.1 & 26 & 0.812 & 0.82 \\
7 & 5.6 & 22 & 0.901 & 0.77 \\
0 & 5.4 & 10 & 0.955 & 0.75 \\
9 & 5.0 & 12 & 1.17 & 0.65 \\
10 & 4.9 & 10 & 1.27 & 0.61 \\
11 & 4.7 & 8.0 & 1.41 & 0.55 \\
12 & 4.5 & 6.0 & 1.60 & 0.49 \\
13 & 4.1 & 5.5 & 1.89 & 0.39 \\
14 & 4.1 & 5.0 & 2.01 & 0.36 \\
15 & 4.1 & 4.5 & 2.11 & 0.33 \\
16 & 4.2 & 4.0 & 2.26 & 0.29 \\
17 & 4.4 & 3.5 & 2.38 & 0.26 \\
18 & 4.6 & 3.0 & 2.60 & 0.20 \\
19 & 4.6 & 2.5 & 2.87 & 0.14 \\
20 & 4.3 & & 3.13 & 0.087 \\
\hline
\end{tabular}

a Observed relative full width half maximum (FWHM).

in Counter I and $45 \mathrm{psi}$ in Counter II. These tables also contain the resolution (FWHM) and relative Gaussian area, $G_{a}$, obtained for each pressure measurement. Figures 12 and 13 show some examples of transformed response functions. Figure 12 presents Counter I results for the 8-, 10-, 14-, and

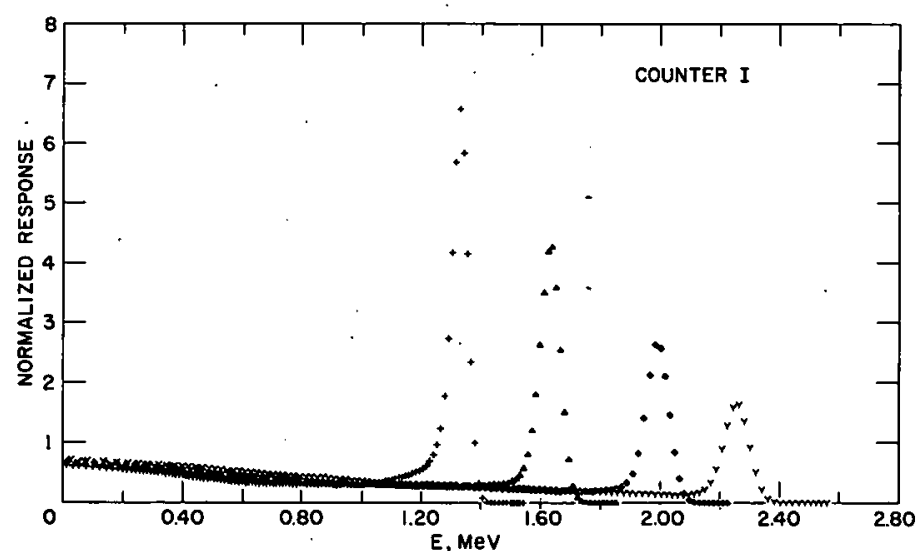

$112-8915$

Fig. 12. Normalized Counter I Response Functions Corresponding to $\mathrm{E}_{0}^{\prime \prime}=2.26,1.99,1.63$, and $1.33 \mathrm{MeV}$ Obtained from Measurements at $8,10,14$, and 20 psi, Respectively
20 -psi measurements corresponding to $E_{0}^{\prime}$ values of $2.26,1.99,1.63$, and $1.33 \mathrm{MeV}$, respectively. Figure 13 presents Counter II results for measurements at 3,4 , and 5 psi corresponding to $E_{0}^{\prime}$ values of $2.87,2.38$, and $2.11 \mathrm{MeV}$, respectively. All transformed response functions have been unit-normalized; i.e., they satisfy the condition

$$
\int_{0}^{\infty} R\left(E^{\prime}, E_{0}^{\prime}\right) d E^{\prime}=1 .
$$




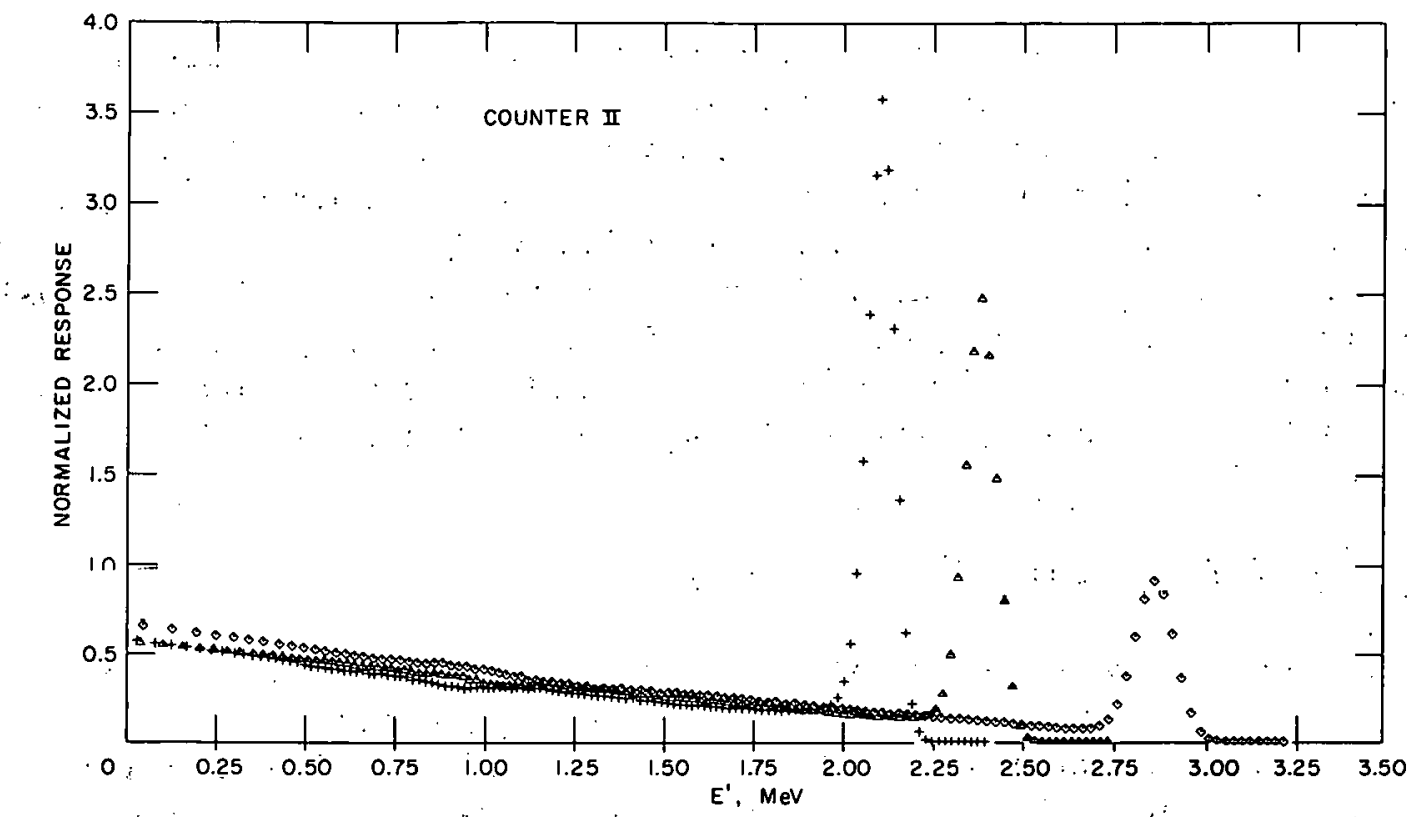

$112-9018$

Fig. 13. Normalized Counter II Response Functions Corresponding to $E_{0}^{\prime}=2.87,2.38$, and $2.11 \mathrm{MeV}$ Obtained from Measurements at 3, 4, and 5 psi, Respectively

The actual behavior of the multiplicative transformation factor in $\mathrm{Eq} . \mathrm{li}$,

$$
\frac{N \cdot \epsilon\left(E_{0}-E\right)}{N^{\prime} \cdot \epsilon\left(\Sigma_{0}^{\prime}-E^{\prime}\right)}
$$

has also been determined for the present experimental data. Figure 14 shows typical results for Counters I and II. As mentioned earlier, these data verify that the multiplicative transformation factor is essentially. constant, except for $E \rightarrow E_{0}$ (or correspondingly $E^{\prime} \rightarrow E_{0}^{\prime}$ ).

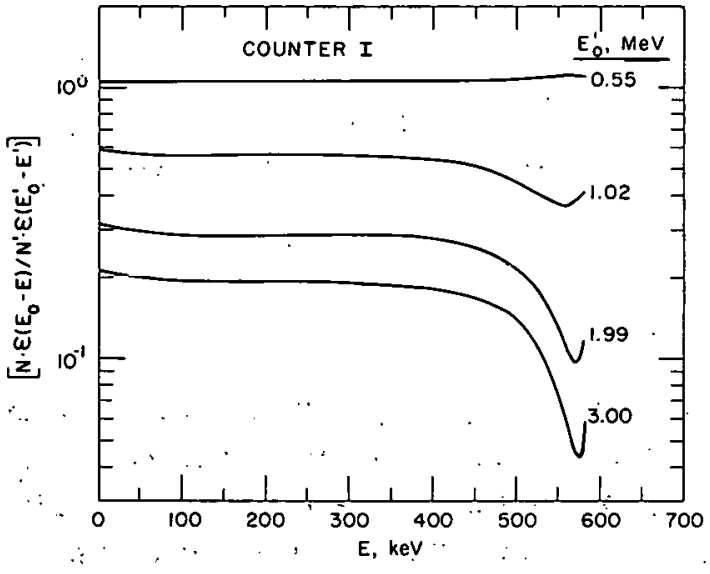

$112-9071$

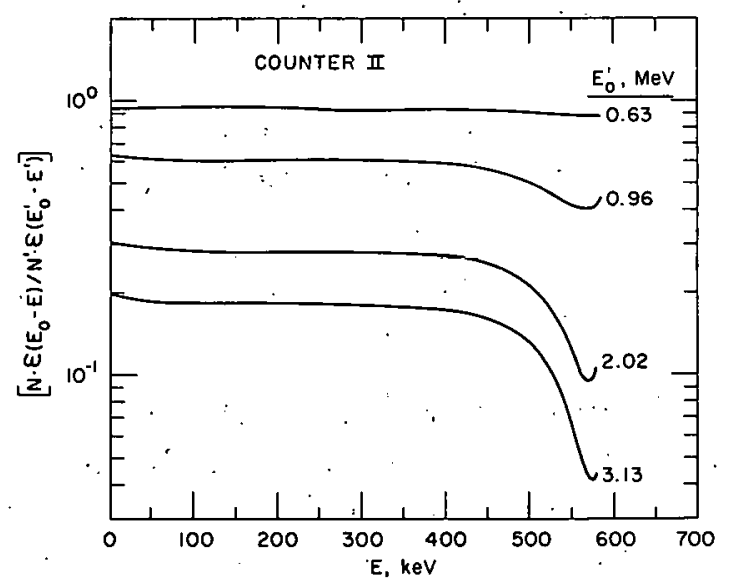

(b)

Fig. 14. The Multiplicative Transformation Factor $N \cdot \epsilon\left(E_{0}-E\right) / N^{\prime} \cdot \epsilon\left(E_{0}^{\prime}-E\right)$ : (a) Counter $I$ at $\mathrm{E}_{0}^{\prime}=0.55,1.02,1.99$, and $3.00 \mathrm{MeV}$; and (b) Counter II at $\mathrm{E}_{0}^{\prime}=0.63,0.96,2.02$, and $3.13 \mathrm{MeV}$ 
The present measurements, or any other experimentally determined set of response distributions, can only serve to define the (energy-dependent) response function on a finite set of points in $E_{0}^{\prime}$-space. On the other hand, construction of the full response matrix requires the continuous behavior of the response function in $E_{0}^{\prime}-s p a c e$. To obtain such a continuous representation, we have introduced two analytical approximations for each measured response distribution. The need for two representations follows naturally from the existence of the distorted and undistorted portions of each distribution. The response function is partitioned accordingly to give

$$
R\left(E^{\prime}, E_{0}^{\prime}\right)=G\left(E^{\prime}, E_{u}^{\prime}\right)+D\left(F_{1}^{\prime}, F_{0}^{\prime}\right)
$$

In this description, a Gaussian approximation, $G\left(E^{\prime}, E_{0}^{\prime}\right)$, was introduced for the undistorted response. The function $\mathrm{D}\left(\mathrm{E}^{\prime}, \mathrm{E}_{0}^{\prime}\right)$, which denotes the distorted response, can now be regarded as the total response minus the undistorted Gaussian response. This is the same representation as was used in the analysis of the $A^{37}$ spectra previously discussed.

form

In the neighborhood of the response peak, a Gaussian fit of the

$$
G\left(E^{\prime}, E_{0}^{\prime}\right)=p\left(E_{0}^{\prime}\right) \exp \left\{-4 \ln 2\left[\frac{E^{\prime}-E_{0}^{\prime}}{\Delta\left(E_{0}^{\prime}\right)}\right]^{2}\right\}
$$

was determined for each measurement. In $E q .15, p\left(E_{0}^{\prime}\right)$ and $\Delta\left(E_{0}^{\prime}\right)$ are the peak value and full width at half maximum of the Gaussian fit. In practice, this fit is obtained by using only those data above the lower half-height energy value, i.e., in the region

$$
E_{0}^{\prime}-\frac{\dot{\Delta}\left(E_{0}^{\prime}\right)}{2} \leq E^{\prime}
$$

The relative Gaussian area, $G_{a}$, listed in Tables I and II was determined on the basis of this Gaussian representation. It is simply.

$$
G_{a}\left(E_{0}^{\prime}\right)=\left(\frac{\pi}{4 \ln 2}\right)^{1 / 2} \cdot p\left(E_{0}^{\prime}\right) \cdot \Delta\left(E_{0}^{\prime}\right)
$$

Given monoenergetic protons of energy $E_{0}^{\prime}, G_{a}\left(E_{0}^{\prime}\right)$ represents (as implied above) the probability of undistorted events arising in the counter. The results summarized in Tables I and II demonstrate that this probability is a rapidly decreasing function of energy. In fact, the undistorted response vanishes rapidly above $3 \mathrm{MeV}$ for both these detectors. 
The resolution values quoted in Tables. I and II have also been determined from this Gaussian representation. The results for both - counters reveal a resolution of approximately $4.5 \%$ for $E_{0}^{\prime} \geq 1 \mathrm{MeV}$. A gradual increase in resolution'sets in below this energy. This behavior can be attributed to counter-gas impurities that contribute to broadening the resolution in the higher-pressure domain:

As pressure increases, proton tracks from the $\mathrm{N}^{14}(\mathrm{n}, \mathrm{p}) \mathrm{C}^{14}$ reaction are correspondingly reduced in length. In Counter $\mathrm{I}$, for example, increasing total gas pressure in excess of about 90 psidoes not produce significant additional changes in the shape of the pulse-height distribution. A substantial componont of the response function which additional pressure increases will not reduce, still exists below the "Gausian peak." The response distribution has reached an "asymptotic" shape; residual effects, especially "tip" effects, are entirely responsible for the non-Gaussian component of these asymptotic distributions. This may be independently. verified by counting the soft (2.8-keV) electrons from $A^{37}$, as described in Part I.B. This radiation has no significant track extension, and the observed pulse-height distribution can only be distorted by nonideal electric field effects associated with the internal construction of the counter. When different intrinsic resolution and background effects are allowed for, the asymp-. totic (high-pressure limit) distribution from the $\mathrm{N}^{14}(\mathrm{n}, \mathrm{p}) \mathrm{C}^{14}$ reaction agrees very well with the distribution measured with the $A^{37}$ source.

The distorted portion of the response, $D\left(E^{\prime}, E_{0}^{\prime}\right)$, was approxi mated by expansion in a Fouricr series. More specifically, the approximation

$$
D\left(E^{\prime}, E_{0}^{\prime}\right) \cong \sum_{k=1}^{N} a_{2 k-1}\left(E_{0}^{\prime}\right) \cos \frac{(2 k-1) \pi E^{\prime}}{2 E_{0}^{\prime}}
$$

was introduced for $E^{\prime} \leq E_{0}^{\prime}$. This set of Fourier functions was chosen to ensure satisfaction of the boundary condition

$$
D\left(E_{0}^{\prime}, E_{0}^{\prime}\right)=0
$$

Experience with the present experimental data has demonstrated that adequate approximations can be obtained from this representation with sixterm expansions $(N=6)$.

In addition to the representation of Eq. 17, the adequacy of two other representations (Legendre polynomials and least-squares polynomials) has been tested. The results obtained with the Legendre expansion were, in general, as adequate as those found with the Foürier series. However, the least-squares polynomial réprésentation was not as satisfactory. Response matrices obtained from this representation did not produce a smooth interpolation of the proton response function for the subsequent iterative unfolding process. 
It is clear why least-squares polynomial fitting is unsatisfactory; each polynomial argument is adjusted, in effect, relative to values assigned to the arguments of all the other polynomial terms. Slight deviations in a distribution may produce large changes in relative arguments. This does not occur in orthonormal expansions, since the amplitude of each member of the set is independently determined.

Two examples of the approximation afforded by Eq. 17 are presented in Figs. 15 and 16 for Counter $I$ at $E_{0}^{\prime}=0.626$ and $3.00 \mathrm{MeV}$, respectively. These figures demonstrate the general trend that occurs; namely, the adequacy of the Fourier representation, Eq. 17, improves with increasing $\mathrm{E}_{0}^{\prime}$. This behavior is quite advantageous, since the distorted portion of the response function increases with increasing $E_{0}^{\prime}$. Consequently, the adequacy of the Fourier representation improves as the (relative) dis torted response area increases.

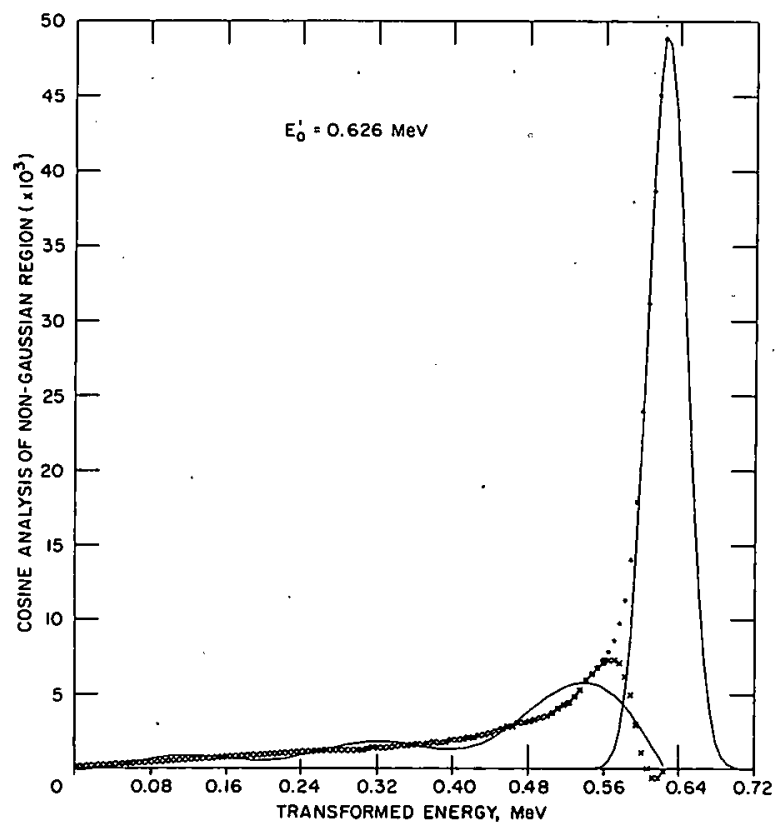

$112-9015$

Fig. 15. Fourier-series Approximations of the Distorted Response Function $D\left(E^{\prime}, E_{0}^{\prime}\right)$ for Counter I at $E_{0}^{\prime}=0.626 \mathrm{MeV}$. The unnormalized Gaussian response at $\mathrm{E}_{0}^{\prime}$ is displayed for orientation purposes only.

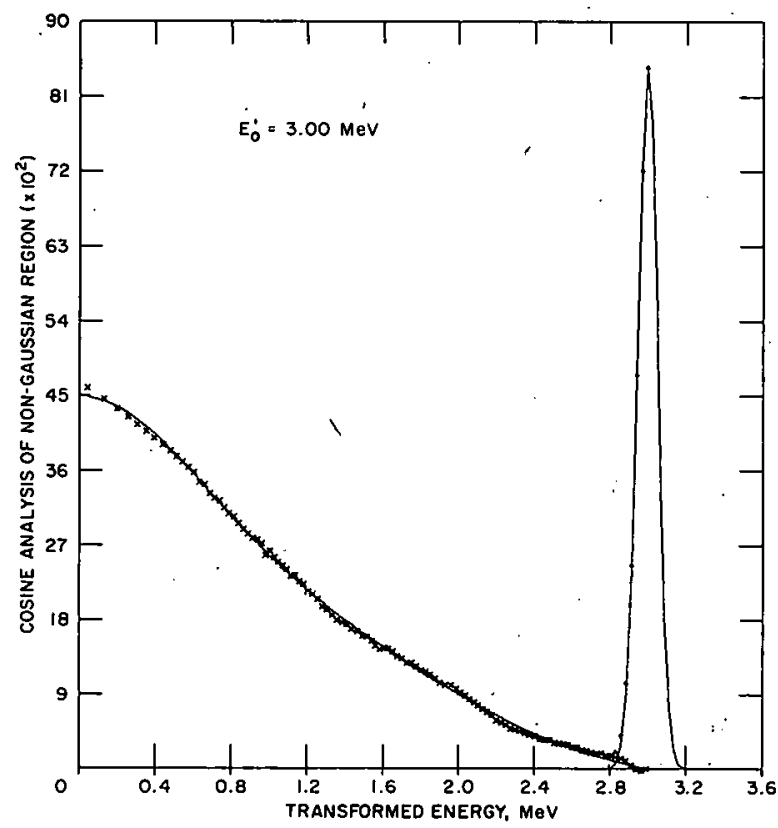

$112-9016$

Fig. 16. Fourier-series Approximations of the Distorted Response Function $\mathrm{D}\left(\mathrm{E}^{\prime}, \mathrm{E}_{0}^{\prime}\right)$ for Counter $\mathrm{I}$ at $\mathrm{E}_{0}^{\prime}=3.00 \mathrm{MeV}$. The unnormalized Gaussiann response at $\mathrm{E}_{\hat{O}}$ is displayed for orientation purposes only.

The basis for a continuous representation of the response function is available from Eqs. 15 and 17. To this end, the parameters $p\left(E_{0}^{\prime}\right)$ and $\Delta\left(E_{0}^{\prime}\right)$ of $E q .15$ and the Fourier coefficients $\left\{a_{2 k-1}\left(E_{0}^{\prime}\right)\right\}$ are all regarded as functions of $E_{0}^{\prime}$. Appropriate analytical approximations of these functions in $E_{0}^{\prime}$-space will then provide the necessary continuous. representation of the response function. 
The process of determining any two-dimensional response function from experimental observations is subject to certain fundamental limitations. The very requirement of a smooth continuous representation of the response function (in $E_{0}^{r}$-space) limits the number of coefficients $\left\{a_{k}\left(E_{0}^{i}\right)\right\}$ that can be used in any chosen representation of $D\left(E^{\prime}, E_{0}^{i}\right)$. In principle, the set $\left\{\mathrm{a}_{\mathrm{k}}\left(\mathrm{E}_{0}^{\prime}\right)\right\}$ can be chosen large enough to obtain any desired degree of approximation to the measured data. (These data represent isolated measurements of the response functions at a limited number of enérgy points in $E_{0}^{\prime}$-space.) However, higher-order coefficients become sensitive not only to experimental error but also to computational procedure and even computer round-off error. It follows that the higher =order cuefficients obtained from any interpolation method will rapidly lose physical significance with increasing order. If too many higher-order terms are retained, this sensitivity will be reflected in the interpolated response function leading to nonsmooth and generally inadequate results. The number of terms used in any given.representation of $D\left(E^{\prime}, E_{0}^{\prime}\right)$ must therefore be a judicious compromise between the complementary needs of employing a good approximation of the response function data and the requirement of a smooth, well-behaved, interpolated response function.

Instead of actually employing $p\left(E_{0}^{\prime}\right)$ and $\Delta\left(E_{0}^{\prime}\right)$ in the continuous representation of the Gaussian response, we used $G_{a}\left(E_{0}^{\prime}\right)$ and $\Delta\left(E_{0}^{\prime}\right)$. This choice is supported by the foregoing discussion, which points out the significance of $\mathrm{G}_{\mathrm{a}}\left(\mathrm{E}_{0}^{\prime}\right)$. Indeed, $\mathrm{G}_{\mathrm{a}}\left(\mathrm{E}_{0}^{\prime}\right)$ is the single most important physical observable associated with the Gaussian response. An adequate continuous approximation of $G_{a}\left(E_{0}^{\prime}\right)$ was obtained with a fourth-order least-squares polynomial fit, and an example of this approximation is presented in Fig. 17.

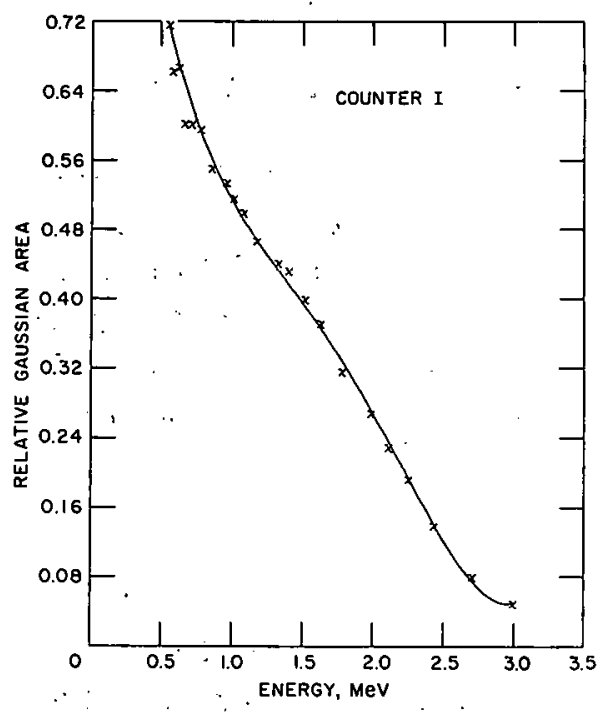

$112-8869$

Fig. 17. Fourth-order Least-squares Polynominal Approximation of Relative Gaussian Areà, $G_{\mathrm{a}}\left(\mathrm{E}_{0}^{\prime}\right)$, for Counter I
In addition, a seventh-order least-squares polynominal fit was used to approximate the behavior of $\Delta\left(E_{0}^{\prime}\right)$. The continuous representation for the parameter $\mathrm{p}\left(\mathrm{E}_{0}^{\prime}\right)$ can then be obtained by using these two polynomial approximations in Eq. 16.

In a similar manner, a least-squares polynomial fit was obtained, for each of the Fourier coefficients, $a_{2 k-1}\left(E_{0}^{\prime}\right)$. Satisfactory continuous representations were obtained with fourth-order polynomial approximations. Typical examples of these approximations are displayed in Figs. 18 and 19, depiciting the behavior of the Fourier coefficients $a_{3}$ and $a_{5}$, respectively, of Counter I.

A particularly advantageous aspect that accrued from the parametrization process was the elimination of erroneous or inconsistent experimental results. This was 
accomplished by examination of any unusually high deviation between a given Fourier coefficient and the corresponding polynomial approximation. If such a deviation arose at a given energy $E_{0}^{\prime}$, the adequacy of all other leastsquares approximations was examined at the same energy. In this manner, we uncovered systematic deviations which implied that the experimental data obtained at a given pressure were suspect and therefore the experiments were repeated.

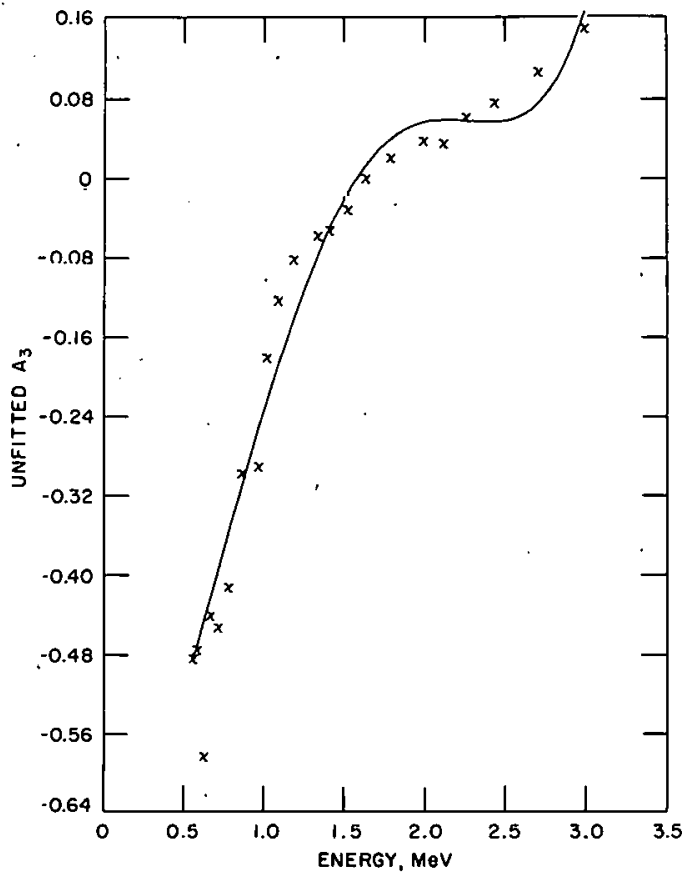

$112-9017$

Fig. 18. Fourth-order Least-squares Polynomial Approximation of Fourier Coefficient $a_{3}\left(E_{0}^{\prime}\right)$ for Counter I

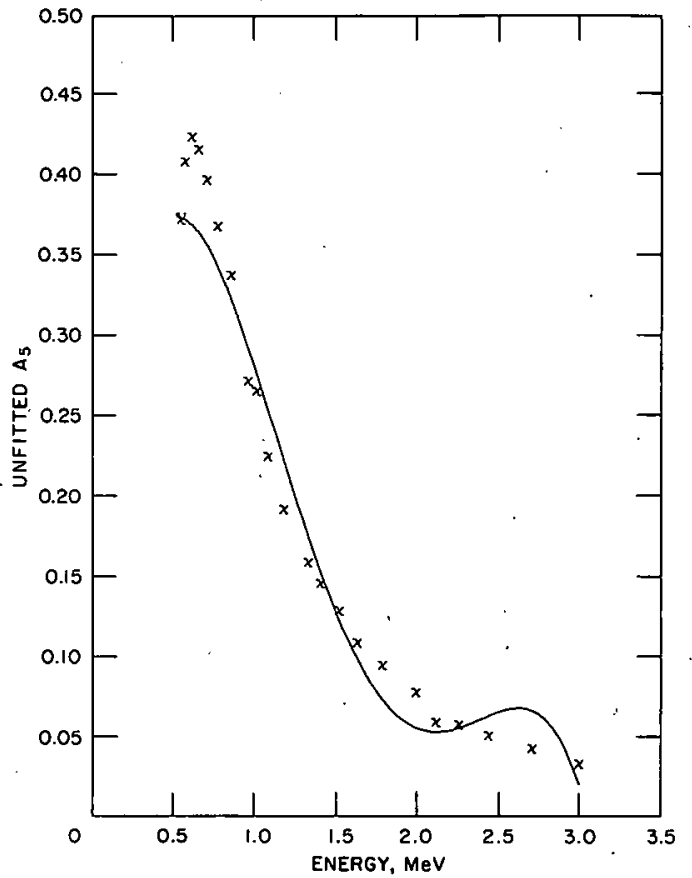

$112-9014$

Fig. 19. Fourth-order Least-squares Polynomial Approximation of Fourier Coefficient $a_{5}\left(E_{0}^{\prime}\right)$ for Counter I

Finally, the parametrized form of the energy-dependent response function must satisfy the low-energy boundary condition. This can be ensured by heavily weighting the parameter values associated with either the asymptotic (high-pressure) or the $A^{37}$ distribution. In the least-squares 'polynomial fitting process of $\left\{a_{k}\left(E_{0}^{\prime}\right)\right\}$ and $G_{a}\left(E^{\prime}\right)$, this heavy weighting will force the approximations to meet the boundary condition. This behavior is clearly demonstrated in Figs. 17-19.

\section{Construction of Response Matrix}

We can now outline the actual steps taken in the construction of a response matrix, using the energy-dependent response function that has evolved from the present numerical analysis of the experimental data.. 
Step 1: Given a response matrix of mesh size A, the midbin energies or midchannel energy points $\left\{E_{j}\right\}$ are generated. The response matrix mesh size $A$ is just the calibrated energy width per pulse-height channel used in the recoil-proton spectral measurement of interest.

Consequently,

$$
E_{i}=A \cdot I_{i}, \quad i=1,2, \ldots, n,
$$

where $I_{i}$ is the integral pulse-height channel number'. The total number of channels so used, n, specifies the order of the response matrix.

Step 2: At each midbin energy, $E_{i}$, a response function, $R\left(E^{\prime}, E_{i}\right)$, is generated using the continuous parametrization described above. One. cannot expect the set of interpolated response functions $\left\{R\left(E^{\prime}, E_{i}\right)\right\}$, as generated, to satisfy Eq. 13 exactly. In practice, the normalization condition is generally satisfied to within a few percent. Consequently, the interpolated response functions are, in turn, normalized so that Eq. 13 is satisfied exactly. In this renormalization process, the relative Gaussian area, $G_{a}\left(E_{i}\right)$, is held invariant, and the area of the distorted response function $D\left(E^{\prime}, E_{i}\right)$ is scaled to meet the unit-area requirement. In this mannér, interpolated response functions retain as closely as possible the behavior of $\mathrm{G}_{\mathrm{a}}\left(\mathrm{E}_{0}^{\prime}\right)$, an observable whose physical significance has been stressed. Figures 20 and 21 display interpolated (renormalized) response functions obtained for Counter I with a matrix mesh of $A=1.9 .53 \mathrm{keV}$ per channel at midbin energies $E_{i}=0.488$ and $2.44 \mathrm{MeV}$, respectively.

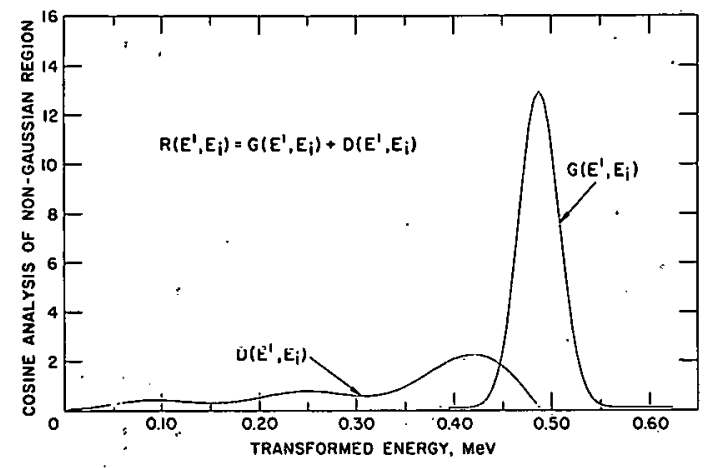

112-8917 Rev. 1

Fig. 20. Interpolated (Renormalized) Response Function $R\left(E^{\prime}, E_{i}\right)$ Obtained from Parametrization Process for Counter I at Midbin Energy $\mathrm{E}_{\mathrm{i}}=0.488 \mathrm{MeV}$

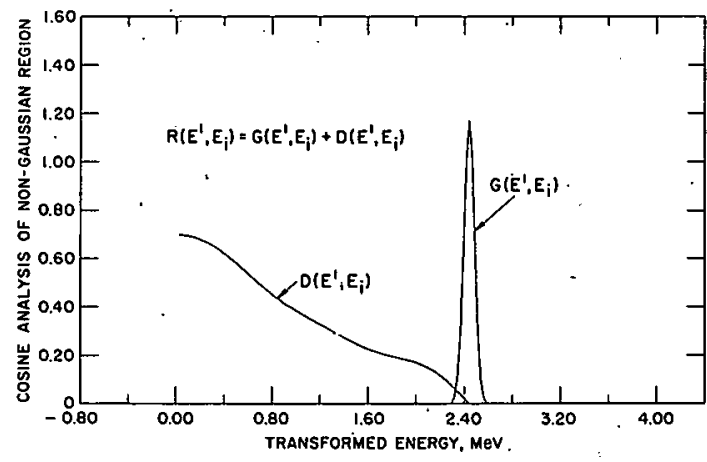

$112-8916 \cdot$ Rev. 1

Fig. 21. Interpolated (Renormalized) Response Function $R\left(E^{\dagger}, E_{i}\right)$ Obtained from Parametrization Process for Counter I at Midbin Energy $E_{i}=2.44 \mathrm{MeV}$

Step 3: In the final step, the response matrix $R$ is formed using the set of interpolated (renormalized) response functions. The responsematrix elements $R_{i j}$ are computed from the customary definition,

$$
R_{i j}=\int_{E_{i}-A / 2}^{E_{i}+A / 2} R\left(E^{\prime}, E_{j}\right) d E^{\prime}, \quad i, j=1,2, \ldots, n .
$$


We have developed a computer program for generating wall effect response matrices (WERM). The WERM code accepts the pressure sequence of experimental measurements and forms the transformed normalized response functions. These transformed response furctions are then parametrized to provide the interpolated response functions: that are used to generate the desired response matrix.

Unfolding with the full-response matrix, as defined in Eq. 20, implies an attempt to remove not only systematic distortion but resolution broadening as well. Fortunately, a treatment of resolution effects is not actually required for proton-recoil data, since we must eventually use a numerical differentiation process to obtain the desired neutron spectrum. Consequently, additional options were included in the WERM code for the generation of two response matrices primarily representative of systematic distortion. These two response matrices (the triangular and delta type) are defined below.

\section{Triangular Response Matrix}

$R_{i j}=0$

$R_{i j}=\int_{E_{i}-A / 2}^{E_{i}+A / 2} R\left(E^{\prime}, E_{j}\right) d E^{\prime}$

$R_{j j}=\int_{E_{j}-\Delta\left(E_{j}\right) / 2}^{\infty} R\left(E^{\prime}, E_{j}\right) d E^{\prime}$

\section{Delta Response Matrix}

$R_{i j}=0$

$R_{i j}=\int_{E_{i}-A / 2}^{E_{i}^{\prime}+A / 2} D\left(E^{\prime}, E_{j}\right) d E^{\prime}$

$R_{j j}=G_{a}\left(E_{j}\right)+\int_{E_{j}-A / 2}^{E_{j}+A / 2} D\left(E^{\prime}, E_{j}\right) d E^{\prime} \quad j=1,2, \ldots, n$.

E. Summary

In Sections $A-D$ above, we have described a variety of experiments which reveal, in considerable detail; the actual counter response function and its variation with energy. These measurements do not, however, constitute a tractable representation of the response function from the viewpoint 
of an unfolding problem. Hence, we have "parametrized" the responsefunction dependence upon energy by a least-squares polynomial fitting - (in powers of energy) of the amplitudes of orthonormal functions derived from each experimental distribution.

The response function must also satisfy a "boundary condition". for sufficiently low proton energy. In this energy region, the response function ceases to depend upon energy and must agree with results obtained from $\mathrm{A}^{37}$ electron counting (where ionization track extent is negligible).

Subsequently, when we speak of the "response function." it will he in the context of a mathematical function determined with arbitrary precision at all energies within the measurement range. We recognize that the parametrized response function will generally exhibit some deviation from the true response distribution; it. will become apparent, . however, that such deviations that do occur do not exert any significant influence in.. neutron spectroscopy. 


\section{UNFOLDING FINITE-SIZE EFFECTS}

A. Introduction

Having determined a suitable response-function representation in Part I, we can now apply it to measurements of proton-recoil spectra. Two methods have been used for unfolding problems of the kind involved here. The first is by repeated iteration; through the response function, of an initial estimate for the unfolded spectrum; arresting conditions terminate the iteration when a satisfactory agreement with the measured distribution is reached. This method is capable of unfolding any appropriate response function and has been used elsewhere. ${ }^{11}$

The second method (integral unfolding) consists of "high-to-lowenergy" integration unfolding and is applicable where the response function retains a significant fraction in the "Gaussian" oregion when the high-energy portion of the spectrum (beyond the measurement limit) is known. This method. has been used previously ${ }^{8}$ for unfolding measured proton-recoil spectra.

We will consider both unfolding techniques, and both, as used here, are subject to systematic error at the upper-energy measurement limit due to the neglect of neutrons that exceed this energy limit. Neutron spectra derived from data unfolded by both these techniques agree well, except near this measurement limit.

\section{B. Iterative Unfolding of Proton-recoil Spectra}

In terms of the response matrix, $R$, the matrix representation of the unfolding problem in the energy-dependent response region takes the form ${ }^{11}$

$$
\underline{W}=\underline{R} \underline{X}
$$

where the $W$ vector represents the measured proton-recoil data, and the appropriate solution, $\underline{X}$, is the (distortion-free) infinite-medium protonrecoil spectrum. Iterative unfolding was used for this system. The computer code, ITERATE, was used for these calculations.

We have already mentioned that use of the full response matrix; viz. Eq. 20, implies unfolding resolution effects that need not.actually be treated (in view of the subsequent numerical differentiation process employed tó extract neutron spectra). Consequently, this presentation will.be confined chiefly to results obtained from delta-type response matrices. Iterative unfolding properties and results obtained with delta and triangular response matrices were virtually identical. A brief examination of iterative 


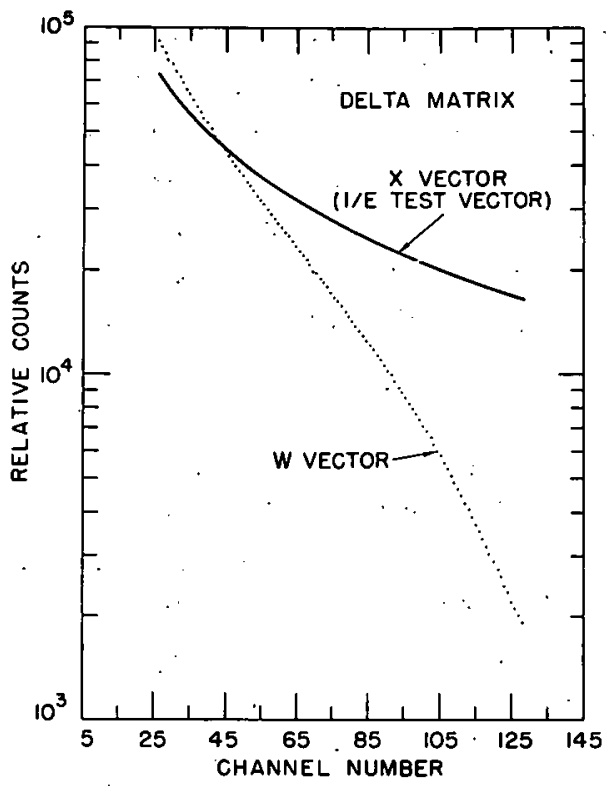

$112-8913$ Rev. 1

Fig. 22. 1/E Test $\underline{X}$ Vector and $\underline{W}$ Veçtor for Counter I Delta-type Response Matrix of Order $n=102$ with Mesh Size of $\mathrm{A}=19.53 \mathrm{keV}$ per Channel unfolding with the full response matrix demonstrated that convergence was slow. and appropriate solutions could not be obtained as readily as with triangular- or. delta-type response matrices.

To test the quality of a generated response matrix, we used a known smooth. $1 / E$ test spectrum as the $\underline{X}$ vector in Eq. 23 . Multiplication of this test vector with the response matrix $\mathrm{R}$ yields an output vector $W$, which can be examined for smoothness. Any deviation of the resulting $\underline{W}$ vector from a smooth monotone behavior implies a.corresponding deficiency in the generated response matrix. Figure 22 presents the $1 / E$ test $X$ vector and the resulting $W$ vector obtained from a delta response matrix of order $n=102$ for Counter I $(A=19.53 \mathrm{keV}$ per channel). The smooth continuous nature of this result indicates that a satisfactory $\mathrm{R}$ matrix has been constructed.
The $W$ vector generated from a chosen $X$ test vector serves another extremely important function. It can be used to investigate convergence properties of the iterative unfolding method. Using this $\mathrm{W}$ vector, we can examine the sequence of iterates $\left\{\underline{x}^{(m)}\right\}$ and thereby directly inspect convergence to the chosen $1 / E$ test spectrum. In addition, we can use the norm of the residual vector, $N[R(m)]$, as a measure of the rate of convergence. (The residual vector is defined as $\underline{R}(\mathrm{~m})=\underline{\mathrm{W}}-\underline{\mathrm{W}}(\mathrm{m})$.)

Figure 23 displays the behavior of $N[\underline{R}(m)]$ for the $1 / E$ test spectrum and the W vector which have been depicted in Fig. 22 . The rate of convergence is very rapid. Moreover, if we assume that the experimental error of the $\underline{W}$ vector is governed by Poisson statistics, the arresting condition is satisfied by the fifth iteration. All iterates, $\{\underline{x}(n)\}$, beyond $n=5$, a re virtually indistinguishable from the $1 / E$ test vector given in Fig. 22. Additional investigations

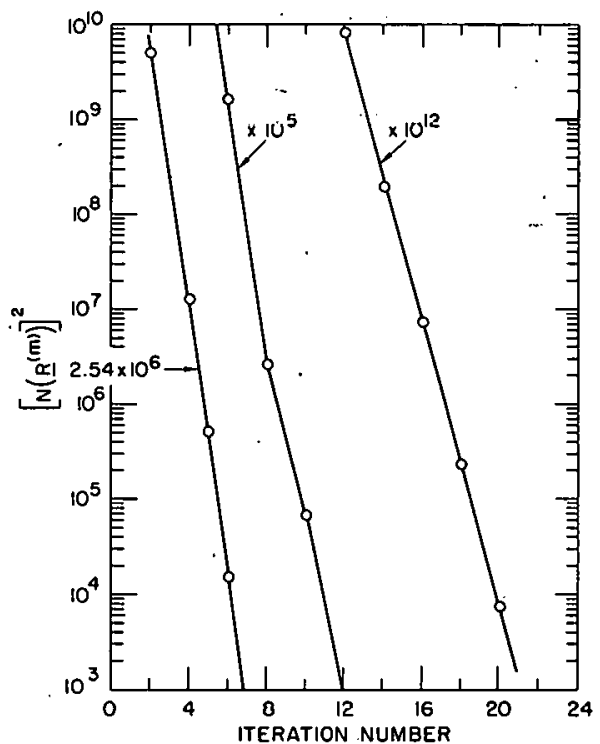

$112-8911$ Rev. 1

Fig. 23. Norm of Residual Vector $\mathrm{N}[\mathrm{R}(\mathrm{m})]$ as a Function of Iteration Number $m$ for the $1 / E$ Test Spectrum and Counter I Response Matrix. (See Fig. 22.) The bound depicted at $2.54 \times 10^{6}$ représents an arresting condition based on Poisson statistics. 
were carried out to test the dependence of these results on the choice of the initial vector $\underline{x}^{(0)}$ (of the iteration process). It has been generally proven that this iteration method furnishes results that are independent of the choice of $\underline{X}^{(0)} .11$ This behavior has been verified for this system by comparison of the cases $\underline{x}^{(0)}=\underline{W}$ and $\underline{x}^{(0)}=$ constant. The convergence properties and solutions obtained with these two different initial vectors were identical. Appropriate solutions are, the refore, clearly determined for this system by iterative' unfolding.

The same delta-type response matrix was employed to unfold actual proton-recoil data measured with Counter I. The $\underline{W}$ vector obtained with Counter $I$ in the neutron spectrum of a fast critical assembly is presented in Fig. 24, along with the resulting unfolded, infinite-medium, protonrecoil spectrum. The measured $W$ vector, which covers the energy region $0.488-2.50 \mathrm{MeV}$, becomes less reliable statistically at the higher-energy end of the spectrum. Note that corresponding statistical uncertainty is reflected in the unfolded solution in the very same higher-energy region. This behavior suggests that the relative error in the unfolded spectrum is approximately the same as the relative error in the measured $W$ vector. Under the present. conditions, this conjecture can, in fact, be more rigorously established. (See Appendix A in this regard.)

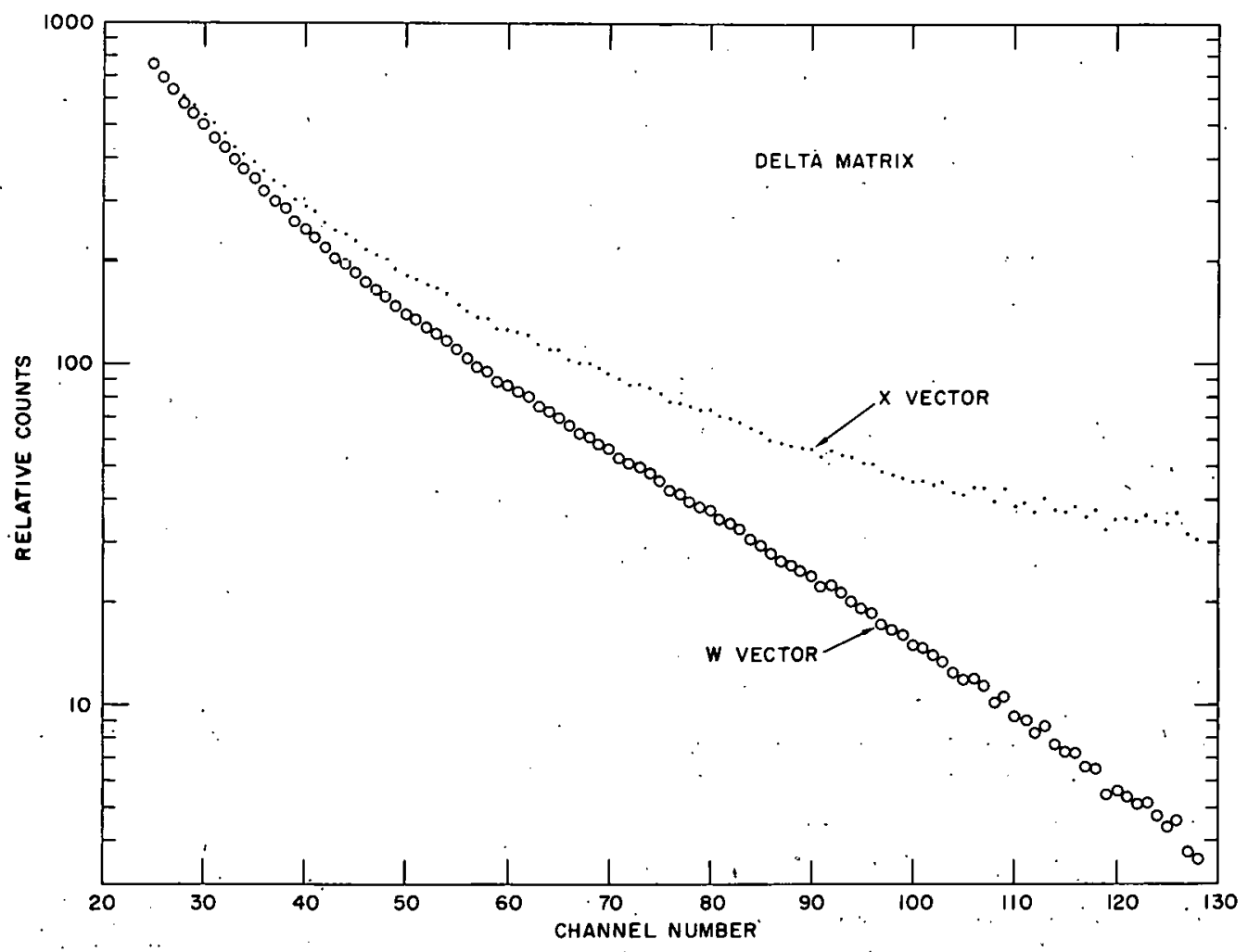

112-9020 Rev. 1

Fig. 24. Infinite-medium Proton-recoil Spectra Obtained from Same W Vector for Delta-type Response Matrix 
Delta- and triangular-type response matrices were compared by generating a triangular response matrix (viz. Eq. 21) to treat the same Counter I proton-recoil data. This triangular response matrix was, therefore, of the same order $\mathrm{n}=102$ and possessed the same mesh size, $\mathrm{A}=19.53 \mathrm{keV}$ per channel. The infinite-medium proton-recoil spectrum obtained by unfolding with this triangular response matrix is presented in Fig. 25. Comparison with Fig. 24 reveals that the solutions obtained with the delta- and triangular-type response matrices are virtually identical. Orily in the lowest and highest few channels is the re any observable deviation between these solutions. The origin of the se differences may be traced directly to the fact that the matrix must be of finite size, or correspondingly cover a finite energy rogion. Consequently, deviations at both the upper and lower extremities of these matrices will be emphasized due to the different definitions of the diagonal matrix elements (viz. Eqs. 21 and 22).

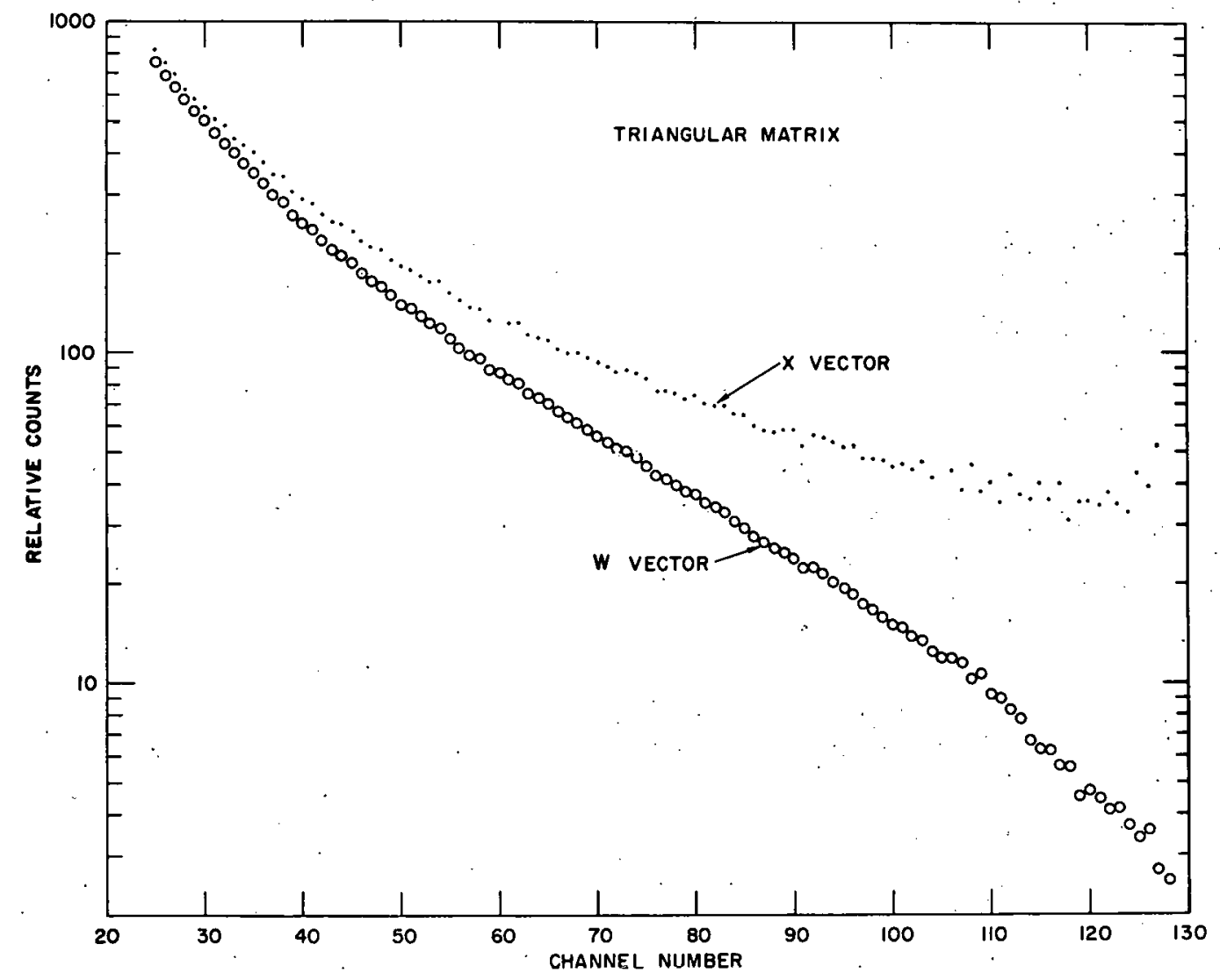

$112-9019$ Rev. 1

Fig. 25: Infinite-medium Proton-recoil Spectra Obtained from Same $W$ Vector for Triangular-type Response Matrix

A more conclusive test of the ability of this procedure to deliver infinite-medium proton-spectra was devised. In this test, the same fastneutron spectrum was measured with two different counters identical in all respects to the geometrical configuration of Counter I depicted in Fig. 7a. These counters differed only in that they were constructed of an 
aluminum body (Counter $\mathrm{I}_{\mathrm{a}}$ ) and a stainless steel body (Counter $\mathrm{I}_{\mathrm{s}}$ ). In addition, Counters $I_{a}$ and $I_{S}$ were filled to methane pressures of 50 and $80 \mathrm{psi}$, respectively. These two detectors were then used to measure the same neutron spectrum in a fast-reactor critical as sembly.

The proton-recoil data or $\underline{W}$ vectors obtained from these two detectors áre compared in Fig. 26, where the measured spectra have been normalized to the same energy width per channel, hydrogen atom density, and experimental live-time. The data for Counter $\mathrm{I}_{\mathrm{a}}$ cover the energy.

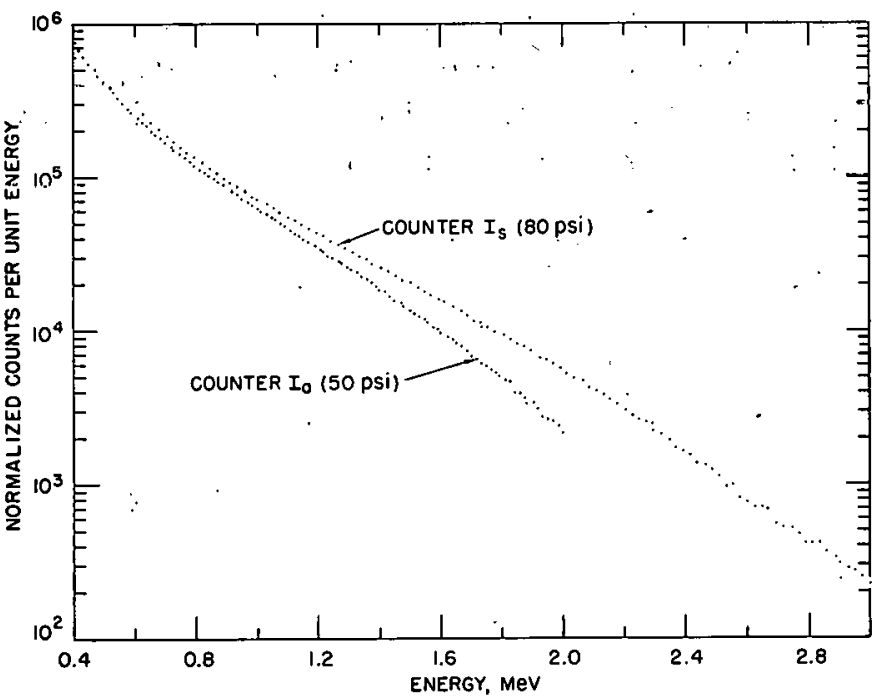

$112-8920$

Fig. 26. Comparison of Measured Proton-recoil Spectra from Counter $\mathrm{I}_{\mathrm{a}}$ (Aluminum Body, 50-psi Methane Filling) and Counter $I_{S}$ (Stainless Steel Body, 80-psi Methane Filling) region $0.4-2.0 \mathrm{MeV}$, while those for Counter $\mathrm{I}_{\mathrm{s}}$ cover the region 0.6-3.0 MeV. This choice was dictated by the fact that the Counter I (energy-dependent) response-function data would generate response matrices in the regions $0.4-2.0 \mathrm{MeV}$ and 0.6-3.0 MeV for the pressure fillings used in Counters $\mathrm{I}_{\mathrm{a}}$ and $I_{S}$, respectively. The difference in the observed proton-recoil spectra, as shown in Fig. 26, demonstrates the anticipated increase in distortion that arises in proton-recoil measurements when counter-gas pressure is reduced.

\section{Delta-type response} matrices were generrated for Counters $I_{a}$ and $I_{s}$ and then used to unfold the corresponding $\underline{W}$ vectors obtained with Counters $I_{a}$ and $I_{S}$. The two infinite-medium proton spectra obtained from this process are compared in Fig. 27. The agreement is quite satisfactory over most of the overlapping energy region.' Above approximately $1.6 \mathrm{MeV}$ the two spectra begin to deviate, and this difference grows with increasing energy. The origin of this relatively minor deviation is, however, readily understood and again stems from the finite size of the response matrix. Truncation of the unfolding process. for Counter $I_{a}$ at $2.0 \mathrm{MeV}$ implies that the neutron flux vanishes beyond $2.0 \mathrm{MeV}$ and therefore leads to anovercorrection in the energy region immediately below $2.0 \mathrm{MeV}$. That is, the finite size of the matrix prevents proper

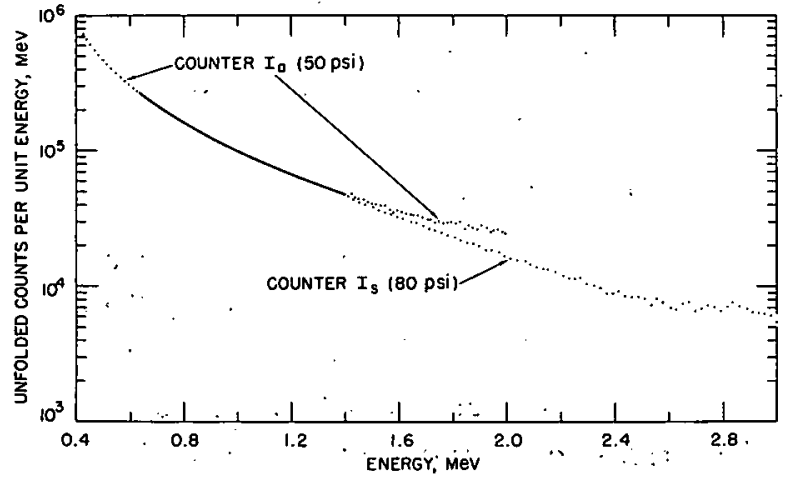

$112-8914$

Fig. 27. Comparison of Unfolded Infinite-medium Proton Spectra Obtained from Counters $I_{\mathrm{a}}$ and $I_{S}$ 
correction of the distortion from being continued. indefinitely (in energy), and in the neighborhood of the high-energy truncation point, the excess proton data that arise from downscattering cannot be completely unfolded.

Thus, the pressure-dependent comparison serves as more than just a test of the validity of the entire unfolding procedure. It also provides a measure of the perturbation that arises at the higher end of the infinitemedium proton spectrum due to the finite size of the response matrix. For the present data, this perturbation is not negligible at energies extending below the high-energy truncation point. It follows that the infinite-medium proton spectra unfolded with finite-size response matrices cannot. he nsed to provide neutron spectra that extend completely out to the high-energy truncation point. One must, instead, be satisfied with neutron spectra that terminate below this truncation point.

C. Integral Unfolding

The integral-unfolding procedure uses the fact that the non-Gaussian component of the finite-size detector response will lie entirely below the dominant peak in nearly every situation encountered. This fact can be used in a "high-low" integral correction procedure which corrects an observed distribution starting at the highest energies and working downward. Knowing the true proton spectrum, $X(E)$, at all energies higher than the energy at which the measured value, $W(E) ;$ is to be corrected, we can remove the distortion to $W(E)$ by using the response function in an integral over $X(E)$. This procedure has been used in previous work, ${ }^{8}$ and it has been. incorporated into the PSNS code. The principal difficulty is that $X(E)$ must be given values in excess of the upper measurement limit where no information exists from the experiment. Fast-reactor spectra.decrease rapidly above the maximum measurement energy (a few $\mathrm{MeV}$ ), and if we take the neutron flux to be zero here, the distortion. is small except near the limit; this point has been demonstrated. in Section II. B.

Consistent with previous notation, let $R\left(E, E_{0}\right)$ denote the detector response to protons of energy $E_{0} \cdot R\left(E, E_{0}\right)$ is subject to the normalization condition given in Eq. 13 and is again partitioned into a dominant term $G_{a} \delta\left(E-E_{0}\right)$ and a non-Gaussian term consisting of a sum of orthogonal functions, where $l-G_{a}$ is the fraction of the response in the non-Gaussian region. $W(E)$, the measured proton. spectrum, can be expressed as

$$
W(E)=\int_{E}^{\infty} X\left(E_{0}\right) R\left(E, E_{0}\right) d E_{0}
$$

Substituting the partitioned response function in Eq. 24 yields 


$$
\ddot{W}(E)=G_{\mathrm{a}} X(\ddot{E})+\int_{E}^{\infty} X\left(E_{0}\right)\left[\sum_{n} \cdot P_{n}\left(E / E_{0}\right)\right] d E_{0}
$$

where $P_{n}$ are the orthogonal terms. Equation 25 can be rearranged to give

$$
X(E)=\frac{W(E)-\int_{E}^{\infty} X\left(E_{0}\right)\left[\sum_{n} P_{n}\left(E / E_{0}\right)\right] d E_{0}}{G_{a}}
$$

The integral term in Eq. 26 involves only values of $X$ ( $E$ ) above the entery $E$, and consequently the correction can be continued downward in energy.

D. Comparison of Unfolding Methods

To illustrate the effect of the two unfolding techniques used here upon a representative reactor spectrum measurement, Fig. 28 compares neutron spectra as derived from proton distributions corrected by both of

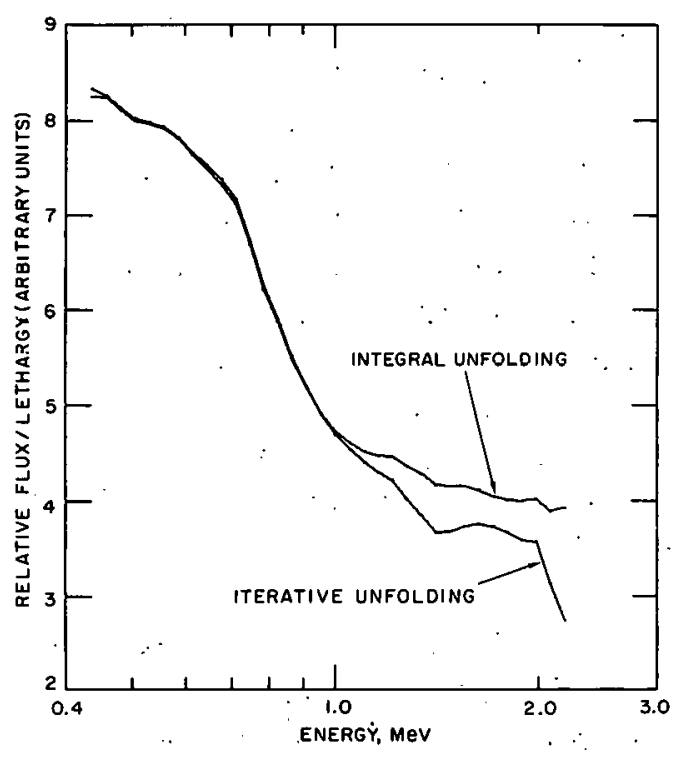

$112-9013$

Fig. 28. Comparison of Neutron Spectra Using Iterative and Integral Unfolding the above-mentioned methods. Below i $\mathrm{MeV}$, agreement is essentially perfect. Above $1 \mathrm{MeV}$, any discrepancy is less than $10 \%$, except at the upper energy limit, where both results are subject to systematic errors.

The results in Fig. 28 are from a. test problem that is explained in detail in Part IV of this report. The raw and unfolded proton spectra are considerably different in this energy region because of the strong energy dependence of the response function. (For example, see Fig. 24.) In spite of the large change in proton spectra at the higher energies caused by unfolding, neutron spectra (in fast reactors) tend to be only moderately influenced. This behavior was not anticipated at the outset of these investigations, and the reason apparently lies partly in the rapidly falling nature of the neutron.flux with increasing energy. The derived neutron flux is considerably more tolerant of response-function detail than can be expected in other spectral environments.

These conclusions are also illustrated by the results shown earlier in Fig. 1. The measured proton distribution is considerably altered as 
energy exceeds about $0.5 \mathrm{MeV}$ by wall-and-end effects; yet the neutron spectra derived from the raw data and from the corrected data are similar.

Although we have considered the two unfolding techniques separately, both can be applied to the same spectrum measurement if so desired. For example, the response information may be expanded to include response distributions for energies above those used in Section II.B. Iterative unfolding would then be essential in the high-energy region; integral unfolding could, however, be applied at the lower energies: 


\section{REDUCTION OF PROTON-RECOIL PULSE-HEIGHT} DISTRIBUTIONS TO NEUTRON-FLUX SPECTRA

\section{A. Introduction:}

The proton-recoil method of neutron spectroscopy is distinguished by the fact that all proton energies below that of the incident neutron energy are equally probable in an $n-p$ collision (at least for neutron. energies below a few MeV). This "rectangular" response has to be "unfolded" from the proton distribution to arrive at the neutron spectrum. The unfolding operation is especially simple, however, consisting of differentiation only where no mitigating circumstances exist. The relationship that converts a proton-recoil distribution $X(E)$, to a distribution in neutron flux (per unit lethargy) $\phi(E)$, is

$$
\phi(E)=-\frac{E^{2}}{N T \sigma_{H^{(E)}}} \frac{d X(E)}{d E},
$$

where $\sigma_{H}(E)$ is the $n \sim p$ scattering cross section, $T$ is the time of accumulation of the $\mathrm{H}$ recoil distribution $\mathrm{X}(\mathrm{E})$ (per unit energy), and $\mathrm{N}$ is the total number of hydrogen atoms in the effective counter.

Equation 27 is a well-established result, and the $n-p$ cross section, $\sigma_{\mathrm{H}}(E)$, can be represented accurately by ${ }^{20}$

$$
\sigma_{H}(E)=\frac{11010}{E+4041}+\frac{2387}{E+135.5}
$$

with $E$ in kilovolts and $\sigma$ in barns. Any departure of $n-p$ scattering from isotropic in the energy region (up to a few $\mathrm{MeV}$ ) accessible to protonrecoil proportional counters is extremely small (not measurable as yet, in fact). The fitting procedures used place Eq. 28 within $2 \%$ of the observed $n-p$ cross section, which has been studied extensively over the energy region of interest.

With a computer to handle the rather lengthy numierical effort, the rectangular response (and the attendant need to differentiate an experimental pulse-height histogram) is a trivial complication to spectroscopy. The existence of a smoothly varying, accurately known, cross-section energy dependence is the most attractive aspect of the proton-recoil method.

The response-function problem and means for eliminating its effect upon measured spectra have been discussed in Parts I and II. In Sections B and $\mathrm{C}$ below, we shall discuss, corrections not involving the response function that are necessary in proton-recoil measurements; in particular, we shall discuss the energy loss per ion pair (W) variation with energy and the distortion of recoil-proton spectra arising from neutron scattering on heavy nuclei (carbon, nitrogen) which may, for various reasons, be present in the counting gas. 


\section{B. Energy per Ion Pair}

If $W$, the energy expended by protons per ion pair created, were constant, proton energy and ionization would be proportional, the constant of proportionality being determined by a calibration. Gases of interest in proton-recoil work (hydrogen and methane) do not exhibit strict proportionality, however. There appears; approximately, to be a threshold energy that must be exceeded before $W$ can be assumed constant. ${ }^{21}$ This threshold in hydrogen is about $0.3 \mathrm{keV}$, and to a good approximation it is the difference $E-0.3 \mathrm{keV}$ that is proportional to pulse height. If $E$ is in excess of $1 \mathrm{keV}$, this simple result should be adequate. In methane, the threshold is more nearly $4 \mathrm{keV}$, if interest is confined to energies above $100 \mathrm{keV}$ or so. The assumption of a threshold cannot be justified except as an approximation to a much more complicated $W$ variation for both gases. It would be desirable to have better models for the $W$ energy dependence, and it is a straightforward procedure to incorporate any improvements into the numerical treatment.

Neutron spectrum measurements have been found to be least ambiguous if hydrogen is used as the counter gas below $100 \mathrm{keV}$ and methane is used above this energy. The influence of $W$ on results is minimized, the additional stopping power of methane can be used to extend measurements to several $\mathrm{MeV}$, and the perturbation of the spectrum due to carbon recoils will be small.

\section{Carbon and Nitrogen Recoils}

Some nitrogen is added to the counters during filling in order to calibrate the energy scale by exposure to a thermal-neutron flux, and the $\mathrm{N}^{14}(\mathrm{n}, \mathrm{p}) \mathrm{C}^{14}$ line at about $615 \mathrm{keV}$ equivalent proton energy is observed. Some methane was also added to the hydrogen counter to quench ultraviolet emission and improve stability. As a consequence, carbon and nitrogen nuclei were present in the counters during measurements. The macroscopic scattering from these heavy nuclei is not insignificant relative to hydrogen and recoils involving the se nuclei contribute to the observed spectrum. A correction for this effect, which is generally small, can be made and has been incorporated into the numerical analysis. We must replace the proton spectrum by the sum of $X(E)$ and $C(E)$, where $C(E)$ represents the spectrum of events originating from carbon recoils. (Similair arguments apply to nitrogen.) Consequently, the measured flux, $M(E)$, is actually given by

$$
M(E)=-\frac{E^{2}}{N_{H} T \sigma_{H}(E)}\left[\frac{d X(E)}{d E}+\frac{d C(E)}{d E}\right],
$$

where $\mathrm{N}_{\mathrm{H}}$ is the hydrogen atom number, and $\sigma_{\mathrm{H}}(\mathrm{E})$ is the corresponding cross section for scattering from hydrogen. The first term on the right 
in Eq. 29 is the true spectrum, and to determine its value, we must make a correction involving the term $\mathrm{C}(\mathrm{E})$. Scattering from carbon and nitrogen is known to be anisotropic for energies above $100 \mathrm{keV}$, and the variation of total cross section with energy is rather complicated. It will be assumed, however, that center -of-mass system scattering is isotropic for both elements and that total cross sections may be represented by the empirical results

$$
\sigma_{C}(E)=\frac{5520}{E+1150}
$$

and

$$
\sigma_{N}(\mathbb{E})=\frac{1250}{E+250},
$$

with the energy $\mathrm{E}$ in $\mathrm{keV}$ and $\sigma$ in barns.

The maximum energy that a carbon recoil can achieve is about $28 \%$ of the incident neutron energy. The relative (to protons) ionization created by a carbon fragment is about 0.75 , however, and therefore the effective ionization of the se heavy nuclei relative to protons is, about $20 \%$. On the basis of these assumptions, the distribution of carbon-recoil events will become

$$
C(E)=5 \int_{E^{\prime}}^{\infty} \phi\left(E^{\prime}\right) \sigma_{C}\left(E^{\prime}\right) \frac{d E^{\prime}}{\left(E^{\prime}\right)^{2}}
$$

If Eq. 31 is used in Eq. 29, the result can be written as

$$
\phi(E)=M(E)-\frac{N_{C}}{N_{H}} \frac{\sigma_{C}(5 E)}{\sigma_{H}(E)} \phi(5 E),
$$

where $\mathrm{N}_{C}$ is the carbon atom number, and $\sigma_{C}$ is the corresponding cross for scattering from carbon, The correction term involves the value of neutron flux at five times the energy at which the correction is made. This correction has been incorporated in the numerical treatment. It is clearly of limited accuracy and should not be used except when small in magnitude. Not only is the treatment of carbon scattering approximate, but it is certain that the relative value of $W$ for heavy nuclei and protons will change appreciably, especially as energy decreases. The correction is, however, generally no more than a few percent, and to this extent Eq. 32 is probably adequate. 
: IV. COMPUTER PROGRAMS.

A: Introduction

The three computer programs WERM, ITERATE, and PSNS are used in the evolution between a measured raw proton-recoil distribution and the final corrected neutron spectrum. Figure 29 shows the logical relationship among these programs. Using the experimental response data, the: W.ERM code generates a parametrized response function for the PSNS code and a response matrix for ITERATE. The order and mesh size of this response matrix are dictated by the spectral measurement. ITERATE calls the respunse matrix, accepts the raw proton data, and provides the unfolded infinite-medium proton spectrum for the PSNS code. Figure 29 shows that an alternate unfolding path is available using the parametrized response function obtained from WERM in the integral unfolding routine of PSNS. Regardless of which path is followed, a routine in PSNS completes the proton-recoil response correction in the energy-independent region. The neutron spectrum and error are determined from the complete infinite-medium proton spectrum by using the PSNS code.

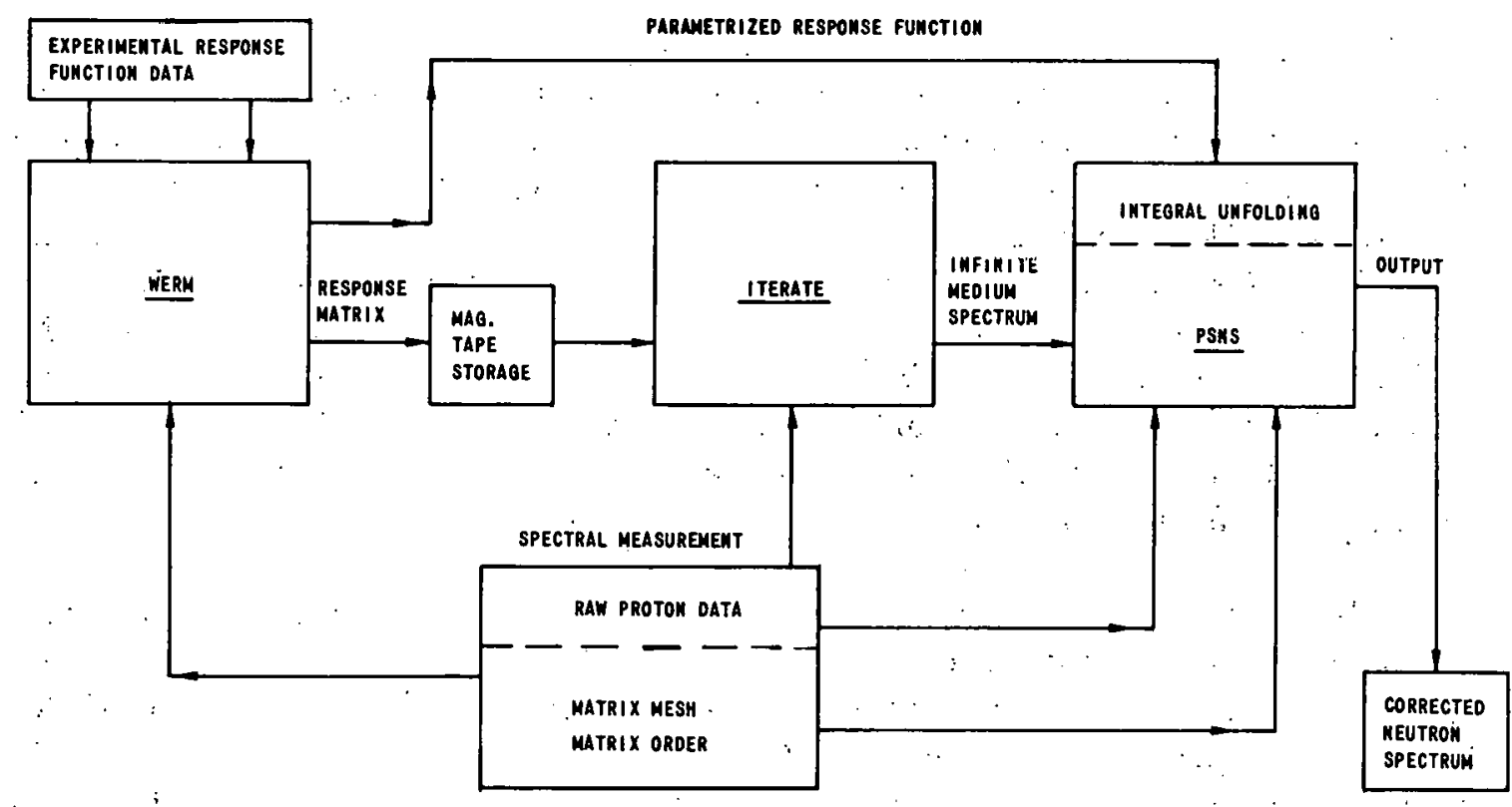

$112-9119$

Fig. 29. Block Diagram of Logical Relationship among Computer Programs WERM, ITERATE, and. PSNS

Sections B, C, and D below contain a detailed exposition of the WERM, ITERATE, and PSNS programs, respectively. A sample problem has been chosen and is traced from beginning to end, i.e., from the raw proton data to the corrected neutron spectrum. A typical fast-reactor neutron-spectrum measurement with a detector similar to Counter I (viz. Fig. 7) has been chosen as this sample case. Required input data are discussed, and examples of computer output are given for this sample 
problem. Different options that are available with these three codes are also described. Detailed input-data requirements and FORTRAN listings of WERM, ITERATE, and PSNS are contained in Appendixes B, C, and D, respectively.

B. The WERM Code

Flow Charts A, B, and C in Figs. 30-32 explain, in logical blockdiagram form, the sequential functioning of the WERM (wall effect response matrix) program: The pressure-dependent response data are treated in Flow Chart A (Fig. 30). Here the pressure sequence of pulse-height distributions is treated one distribution at a time, in order of decreasing pressure. Each distribution is fitted to a Gaussian in the neighborhood of the response peak by calling the VMM (variable metric minimization) subroutine. ${ }^{22}$ Provision is made for bypassing the Gaussian fitting procedure if it has been previously performed with the same response data. In this event, the previously determined Gaussian parameters $\left(X_{1}, X_{2}\right.$, and $X_{3}$ ) must be read into WERM as input data. Table III is a typical computer output of this Gaussian fitting procedure, which is available as an option in WERM.

Subtraction of this Gaussian leaves the distorted portion of the response data, which is treated by the energy-density transformations developed in Section I.C and depicted in Flow Chart A. The set of transformed energy values $\left\{E_{0}^{\prime}\right\}$ (designated by EP(O) in the code output) as well as the relative Gaussian area, $\mathrm{G}_{\mathrm{a}}$, for the complete pressure sequence can be obtained in the form displayed in Table IV, and is printed out when the VMM Gaussian fitting procedure is bypassed. 'Table V summarizes other pertinent output data resulting from the Gaussian fitting routine. In the final step of Flow Chart A, the Fourier-series approximation of the transformed (difference) data is obtained.

Flow Chart B (Fig. 31) depicts the sequence of computations that lead to the parametrization of $G_{a}\left(E_{0}^{\prime}\right)$ and the Fourier coefficients $\left\{a_{k}\left(E_{0}^{\prime}\right)\right\}$. Table VI, contains typical output of the least-squares polynomial (LSQPOL) coefficients obtained in fitting both $\mathrm{G}_{a}\left(\mathrm{E}_{0}^{\prime}\right)$ and the set $\left\{\mathrm{a}_{\mathrm{k}}\left(\mathrm{E}_{0}^{\prime}\right)\right\}$. The midbin energies $\{E O F(I)\}$ for the response matrix are created using the matrix mesh $A$ as well as the low and high pulse-height channel cutoffs (LOWCH and $\mathrm{MAXCH}$ ) of the spectrum measurement. The interpolated Gaussian parameters $X_{1}[\operatorname{EOF}(I)]$ and $X_{2}[\operatorname{EOF}(I)]$, as well as the interpolated Fourier coefficients $\left\{a_{k}[E O F(I)]\right\}$, are formed and stored in computer memory. Then interpolated Fourier coefficients are scaled [by the scale factor $\mathrm{SC}(\mathrm{N})$ ] to preserve the unit-area constraint for the interpolated response distribution. A printout option is available for all these interpolated parameters. 


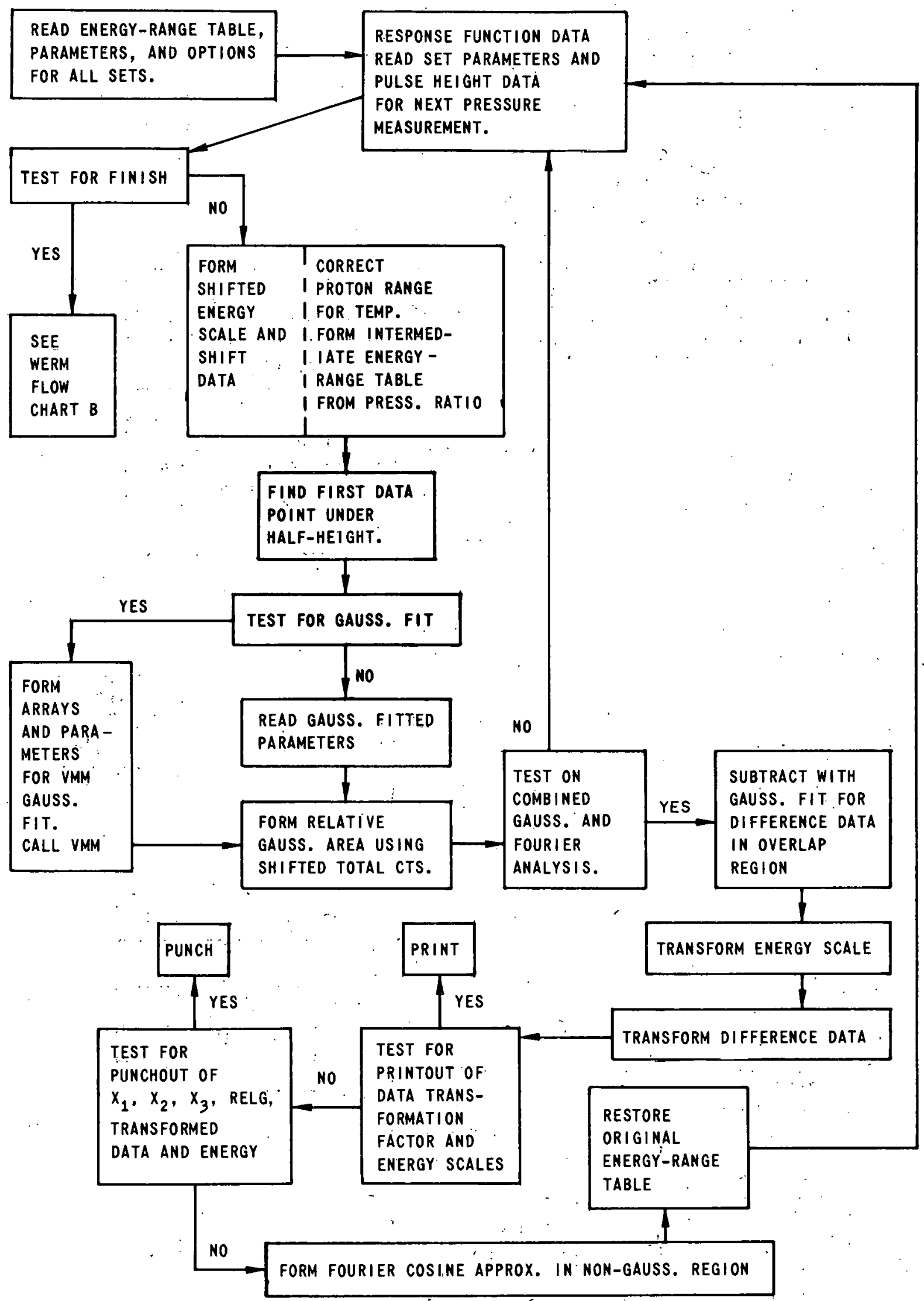

112-9124 Rev. 1

Fig. 30:: Flow Chart A for WERM Code, Showing Treatment of Pressure -dependent Response Data 


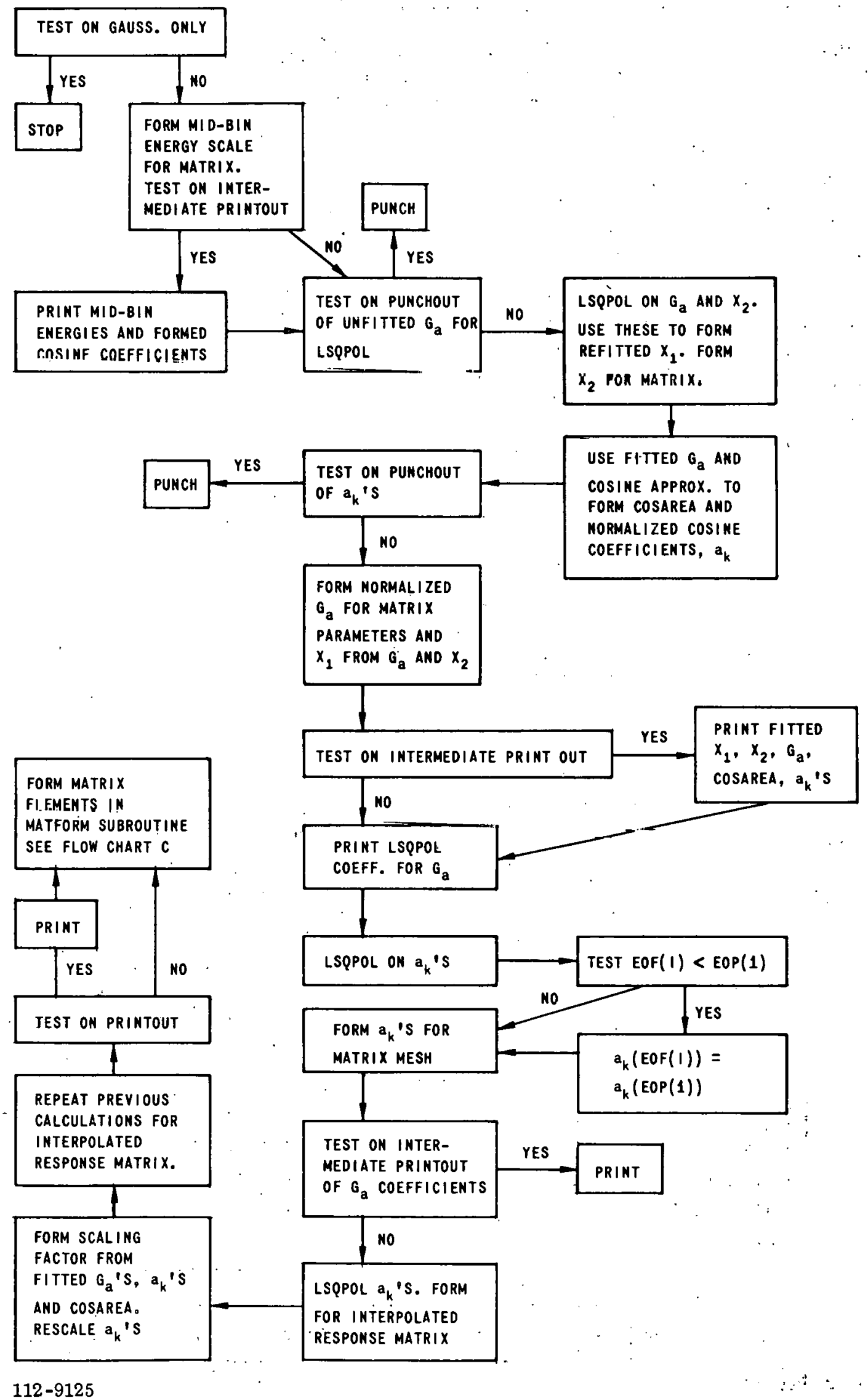

Fig. 31. Flow Chart B for WERM Code, Showing Computations Leading to Parametrization of: $G_{a}\left(E_{0}^{\prime}\right)$ and Fourier Coefficients $\left\{a_{k}\left(E_{0}^{\prime}\right)\right\}$ 


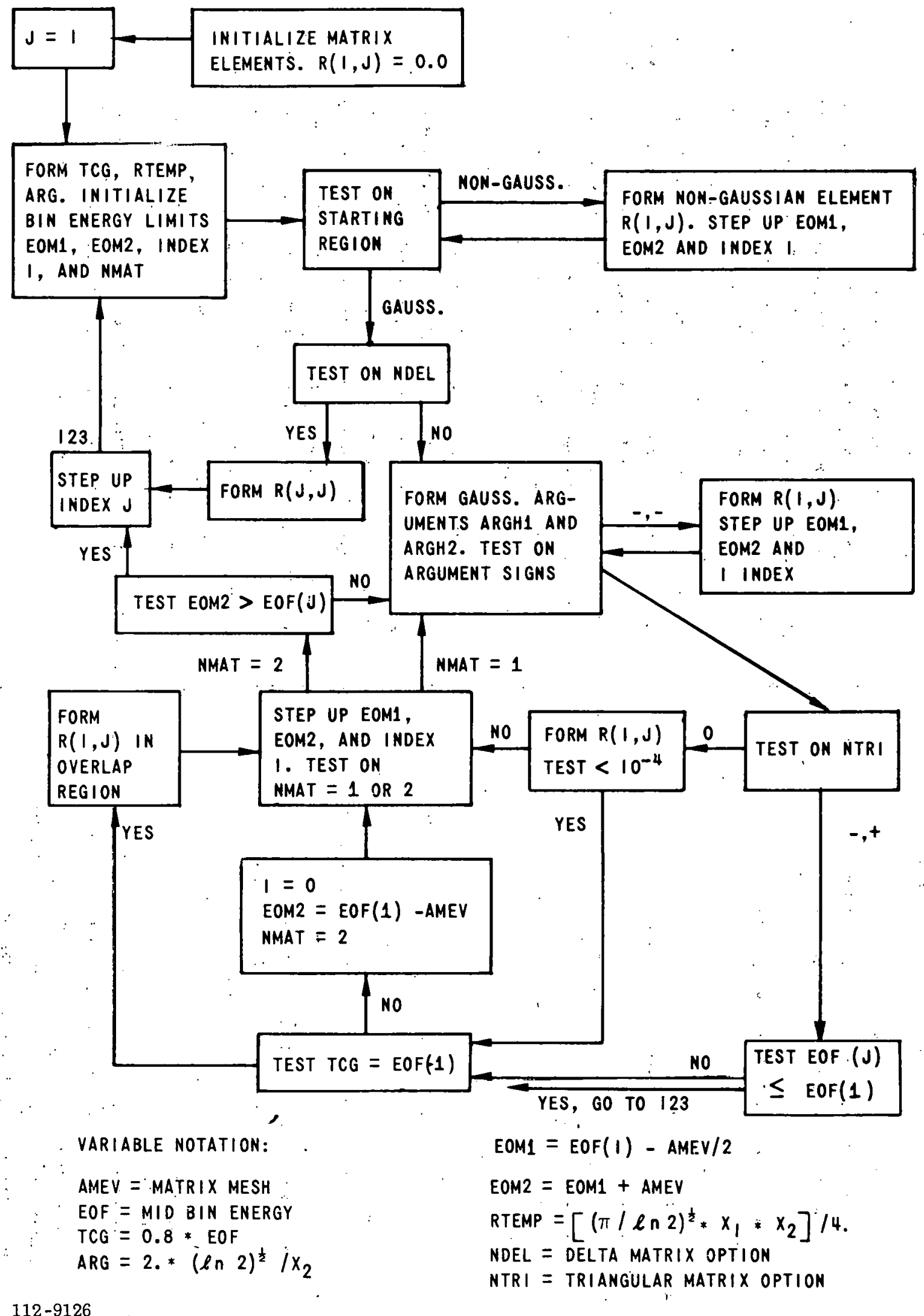

$12-9126$

Fig. 32. Flow Chart C (MATFORM Subroutine) for WERM Code, Showing Formation of Matrix Elements 
TABLE III. Output of Gaussian Fitting Routine (VMM Da:a)

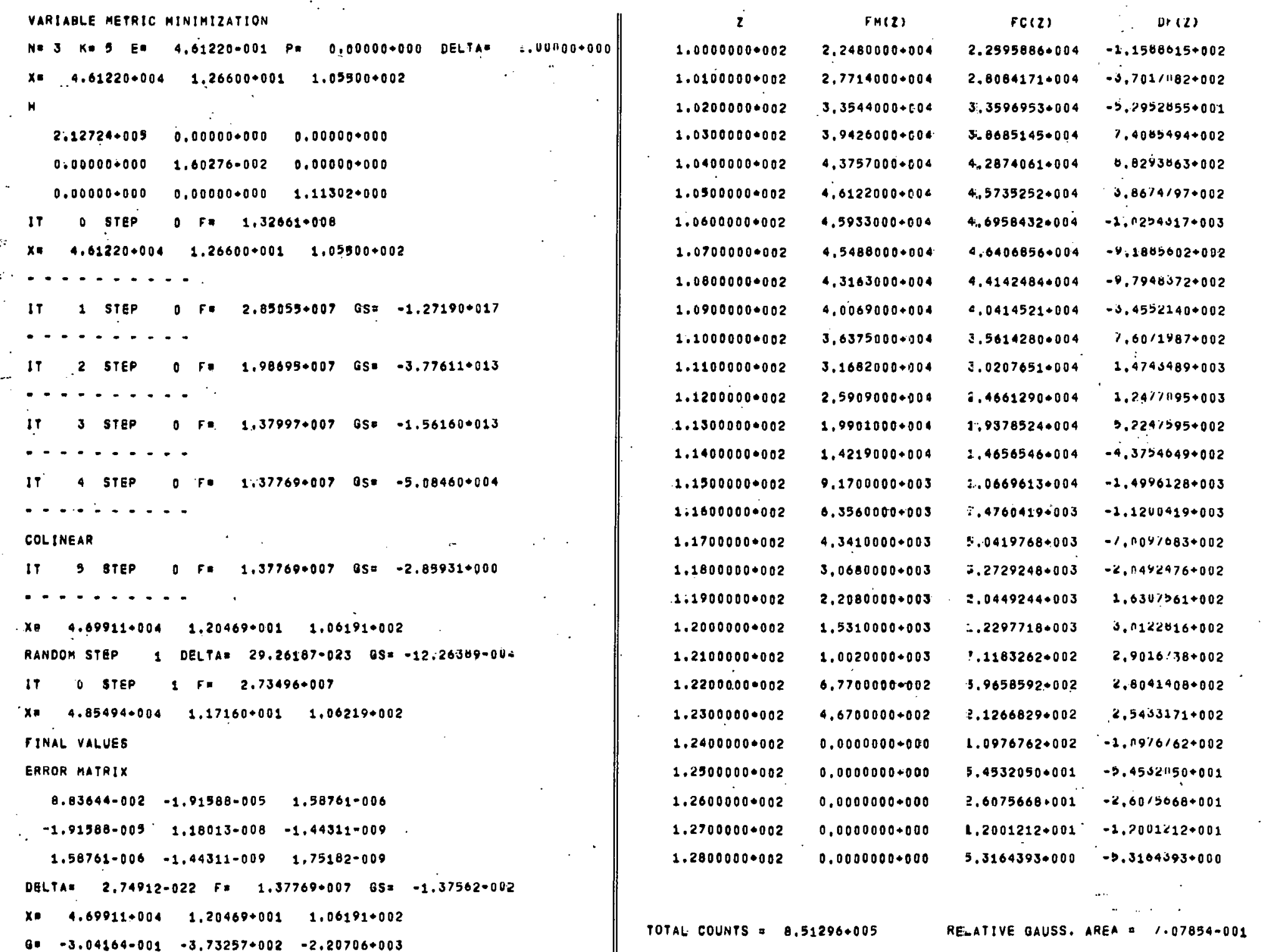


TABLE IV. Summary of Gaussian Data for Pressure Sequence

\begin{tabular}{cccc}
\hline Transformed Energy & $\begin{array}{c}\text { Total Counts } \\
\text { (Raw Data) }\end{array}$ & $\begin{array}{c}\text { Relative } \\
\text { Gauss. Area }\end{array}$ \\
\hline $\mathrm{N}$ & EOP(N) & $8.51296+005$ & 0.707853 \\
1 & 0.55645 & $8.21928+005$ & 0.653221 \\
3 & 0.58336 & $6.45134+005$ & 0.658141 \\
4 & 0.62573 & $7.44860+005$ & 0.592866 \\
5 & 0.66758 & $8.07871+005$ & 0.592001 \\
6 & 0.71944 & $1.15094+006$ & 0.586428 \\
7 & 0.78043 & $9.68506+005$ & 0.541008 \\
8 & 0.86492 & $6.48913+005$ & 0.525489 \\
9 & 0.96616 & $9.11205+005$ & 0.506667 \\
10 & 1.01705 & $7.78049+005$ & 0.490199 \\
11 & 1.08827 & $1.10599+006$ & 0.457028 \\
12 & 1.18490 & $7.24729+005$ & 0.431909 \\
13 & 1.33164 & $6.05536+005$ & 0.422719 \\
14 & 1.40678 & $6.51792+005$ & 0.390157 \\
15 & 1.52059 & $9.04207+005$ & 0.361804 \\
16 & 1.63459 & $8.34195+005$ & 0.307011 \\
17 & 1.78693 & $8.55170+005$ & 0.259141 \\
18 & 1.99294 & $7.87187+005$ & 0.219275 \\
19 & 2.11579 & $8.13380+005$ & 0.182609 \\
20 & 2.25737 & $7.46361+005$ & 0.129182 \\
21 & 2.43928 & $1.20477+006$ & 0.070559 \\
22 & 2.70749 & $9.96482+005$ & 0.040649 \\
\hline & 2.99581 & &
\end{tabular}

TABLE V. Summary of Gaussian Parameters for Pressure Sequence

\begin{tabular}{|c|c|c|c|c|c|c|c|}
\hline \multirow{2}{*}{\multicolumn{2}{|c|}{$\mathrm{N}$}} & \multicolumn{4}{|c|}{ Fitted Gaussian Parameters } & \multirow[b]{2}{*}{$\operatorname{FITG}(\mathrm{N})$} & \multirow{2}{*}{ Cosine Area } \\
\hline & & $\mathrm{x}(3)$ & $=\mathrm{EOP}, \mathrm{MeV}$ & $\mathrm{X}(1)$ & $x(2)$ & & \\
\hline 1 & & & 0.55645 & 10.53630 & 0.063025 & 0.70686 & $1.46749+003$ \\
\hline 2 & & & 0.58336 & $1 \mathrm{i} .45969$ & $0.0 \dot{5} 6523$ & 0.68949 & $1.54074+003$ \\
\hline 3 & & & 0.62573 & 12.75326 & 0.048903 & 0.66388 & $1.17834+003$ \\
\hline 4 & & & 0.66758 & 13.69430 & 0.043942 & 0.64054 & $1.52295+003$ \\
\hline 5 & & & 0.7 .1944 & 14.24327 & 0.040502 & 0.61408 & $1.62285+003$ \\
\hline 6 & & & 0.78043 & 14.03646 & 0.039226 & 0.58609 & $2.26009+003$ \\
\hline $7^{\prime}$ & & & 0.86492 & 12.78666 & 0.040566 & 0.55215 & $2.07335+003$ \\
\hline 8 & & & $.0: 96616$ & 10.91043 & 0.044562 & 0.51753 & $1.40471+003$ \\
\hline 9 & & & 1.01785 & 10.03106 & 0.047004 & 0.50189 & $2.07176+003$ \\
\hline 10 & & & 1.08827 & 8.98629 & 0.050424 & 0.48233 & $1.83011+003$ \\
\hline 11 & & & 1.18490 & 7.82119 & 0.055011 & 0.45799 & $2.69740+003$ \\
\hline 12 & - & & 1.33164 & 6.47516 & 0.061264 & 0.42434 & $1.96915+003$ \\
\hline 13 & & & 1.40678 & 5.91034 & 0.064815 & 0.40777 & $1.68240+003$ \\
\hline 14 & . & & 1.52059 & 5.15191 & 0.069761 & 0.3825 .7 & $2.00034+003$ \\
\hline 15 & & & 1.63459 & 4.47528 & 0.074814 & 0.35640 . & $2.92021+003$ \\
\hline 16 & & & 1.76693 & 3.67267 & 0.081579 & 0.31893 & $2.97256+003$ \\
\hline 17 & the & & $1.99294^{\circ}$ & 2.74492 & 0.089967 & 0.26287 & $3.38192+003$ \\
\hline 18 & & & 2.11579 & 2.26188 & 0.094232 & 0.22688 & $3.22596+003$ \\
\hline 19 & $\therefore$ & & 2.25737 & 1.75419 & 0.098646 & 0.18420 & $3.64164+003$ \\
\hline 20 & . & & 2.43928 & 1.17008 & 0.104740 & 0.13046 & $3.62261+003$ \\
\hline 21 & & & 2.70749 & 0.52098 & 0.117954 & 0.06541 & $6.45152+003$ \\
\hline 22 & & & 2.99581 & 0.31853 & 0.123515 & 0.04188 & $5.74316+003$ \\
\hline
\end{tabular}


TABLE VI. Least-squares Polynomial Coefficients for Fourier Coefficients and Gaussian Area

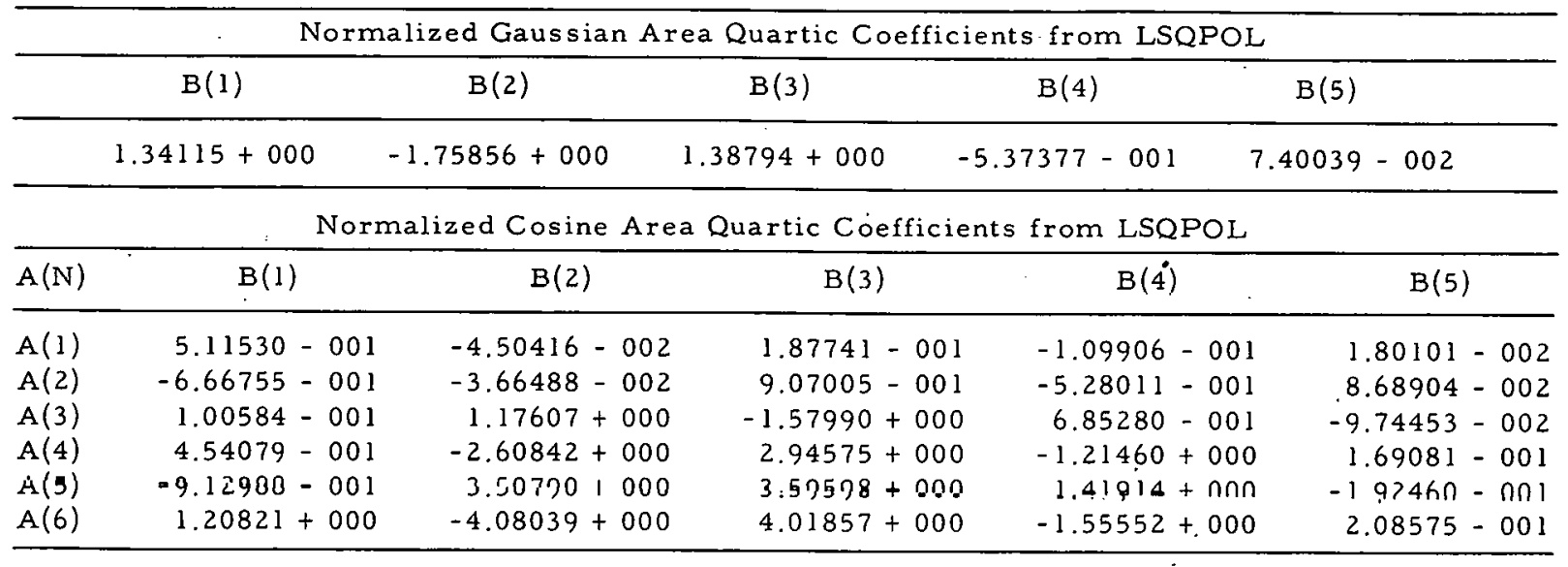

The process of generating a response matrix is described in Flow Chart C (Fig. 32), which considers the logical order of computations in subroutine MATFORM. The square matrix $R(I, J)$ is initialized as the null matrix and then is constructed column by column, working from Column 1 (LOWCH) up to Column $\mathrm{n}(\mathrm{MAXCH})$. In each column, the matrix elements are formed by working from Row 1 to Row $n$. Matrix elements for each column are computed by integration of the response function over fixed energy intervals. The parametrized response function associated with a given column is retrieved from computer storage, and the fixed energy interval of integration (viz. Eq. 20) is determined by the row location. The parametric representation of the response function changes with row location from the non-Gaussian or distorted region, to an overlap region, and then to the purely Gaussian region. The variable TGG $=0.8 \mathrm{EOF}(\mathrm{J})$ has been introduced to define the start of the overlap region for Column $\mathrm{J}$, i.e., that region in $E_{0}^{\prime}$-space where both Fourier and Gaussian representations must be simultaneously employed. (This overlap region ends at the energy $\operatorname{EOF}(\mathrm{J})$. )

The input parameters NDEL and NTRI allow different types of response matrices to be generated. For NDEL = NTRI = 0, the full-response matrix is constructed. The choice NDEL $\neq 0$, NTRI $=0$ leads to a deltaresponse matrix; and NTRI $\neq 0$, NDEL $=0$ yields a triangular-response matrix.

For most response matrices of interest, the order $\mathrm{n}$ is quite large. Hence provision has been made to store the matrix generated by subroutine MATFORM on magnetic tape. In this manner, the matrix is accessible and can be readily called by the program ITERATE. The entire matrix can be printed out. Tables VII and VIII present the upper and lower corners of the delta response matrix generated for the sample problem, where $\mathrm{A}=$ $0.0179 \mathrm{MeV}$ per channel and $\mathrm{n}=98$ (i.e., $\mathrm{L} \Theta W C H=31, \mathrm{MAXCH}=128$ ). 
TABLE VII. Printout of Upper Corner of Delta Response Matrix for Sample Problem

MID ENERGY (MEV)

\section{$1 / \mathrm{J} \perp 0.5549$}

7.06860001

0,00000000

0,00000000

0.00000000 .

0.00000000

0.00000000

0.0000 .000

0.00000000

0.00000000

0.00000000

0.00000000

$0,0000+000$

$8.0000+000$

$0,0,000000000$

$0.0000+000$

0.00000000

$0.0000 * 000$

$0.0000 \times 000$

0,00000000

$0,0000 * 000$

$0.00000 * 000$

$0.0000+000$

$0.0000 \times 000$

0.00000000

0,00000000

0,00000000

0.00000000

0.00000000

0.00000000

$0,0000 \times 000$

0,00000000

$0.0000 * 000$

$0.0000 \times 000$

0.00000000

0.00000000

0.000000000

0.00000000

0.00000000

0,00000000

0.00000000

$0.0000 \times 000$

0.00000000

0.0000000

0.00000000

0.00000000

$0.0000 \times 000$

0.00000000

$0.0000+000$

0.000000000

$0.0000 \times 000$

0.00000000

$0,0000 \times 000$

0.00000000

0.00000000

0.00000000

$0.0000 \times 000$

$0,0000<000$

0,00000000

0.00000000

0,00000000

0.0000 .000

0.00000000

0.00000000

0,00000000

0.00000000

0.000000000

0.00000000

0.00000000

$0.0000 \times 000$

0.00000000

0.00000000

0.00000000

0.00000000

0.00000000

0,00000000
RESPONSE HATRTX

\begin{tabular}{|c|c|}
\hline 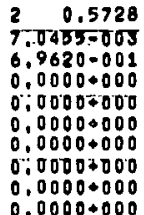 & 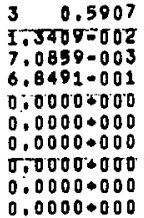 \\
\hline
\end{tabular}

$0,0,0000 \times 000$

$0.0000+000 \quad 0.0000+000$

$0.0000+0000000000000000000$

$0.0000+000 \quad 0.0000 \times 000$

$0.0000+000 \quad 0.0000+000$

$\begin{array}{ll}0,00007000 & 0,00000+000 \\ 0.0000000 u & 0,0000+000 \\ 0.0000+000 & 0,00000000\end{array}$

$0,0000+000$

$0.0000+000$

$0,0000 \div 000$

$0.0000+000$

$0,00000.000$

$0,0000+000$

$0,0000+000$

$0,0000+000$

$0,0000+000$

$0,00000 \times 000$

$0.0000+000$

$0 \div 0000+000$.

$0.0000+000$

$0,0000+000$

$\sigma .0000+000$

$0,0000 \times 000$

0,00000000

$0.0000+000$

0,00000000

$0.0000+000$

0,00002000

$0,0000+000$

$0,0000+000$

0.00000000

$0,0000+000$

$0.0000+000$

$0.0000+000$

$0,0000 \% 000$

$0.0000+000$

0.00000000

0.00000000

$0,00000+000$

$0.0000+000$

$8.0000+000$

$0.0000+000$

$0.0000 \times 000$

$0.0000+000$

$0,0000+000$

0.00007000

$0,0000+000$

$0: 0000 \cdot 000$

$0.0000+000$

0.00005000

$0.0000 \times 000$

0.000007000

$0.0000+0.00$

$0,0000 \times 000$

$0,0000 \times 000$

$0.0000+000$

0,00000000

0,00000000

0.00000000

$0.00000 \times 000$

0.00000000

$0.00000+000$

$0,00000+000$
$0.0000+000$

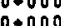

$0,0000+000$

$0.0000 \times 000$

$0.0000 \times 000$

$.0000 \cdot 000$

$0.0000 \times 000$

$0,0000 \times 000$

$.0000+000$

$0.0000+000$

$0,0000+000$

$0,0000+000$

$0,0000+000$

00004000

$0.0000+000$

$00000+000$

$0.0000 \times 000$

$0.0000+000$

$0.0000+000$

$0.0000+000$

$0.0000+000$

0.00000000

0.0000 .000

$0.0000+000$

0.00000000

$0.0000+000$

0.00000000

$0,00000 \times 000$

$0.0000+000$

0.00000000

0.00000000

$0,0000+000$

$0.0000+000$

$0.0000+000$

$0.0000+000$

$0.0000+000$

$0,0000+000$

$0.0000+000$

$0 \% 0000+000$

$0.0000+000$

$0,0000+000$

$0,0000 \times 000$

$0.0000+000$

$0.0000+000$

$0,0,000 \times 000$

$0,0000+000$

$0,0000+000$

$0,0000 * 000$

0,00000000

$0.0000 \times 000$

$0.0000+000$

$0,0000 \bullet 000$

$0.0000+000$

0.0000 .000

$0.0000+000$

$0 ; 0000+000$

$0.0000 \times 000$

$0.0000+000$

0.000000000

0.00000000

$0,0000+000$

0.00000000

\section{$4 \quad 0.6786$}

1308200102

$7 \div 094 \%-1102$

$6=7304-1101$

$00000+1100$

$0: 0 n 00+1100$

$0.0 n 04+1100$

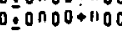

oronote+100

$0.0 n 00+1100$

grovatido

$0.0000+1100$

$0.0 n 00+1700$

oipnopenn

$0=0 n 00+100$

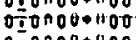

$0: 0 n 00+1100$

$00 r 00+f 00$

$0=0 n 00+1100$

$0.0000+100$

$0=0 n 00+1100$

$0 \leq 0 n 00+1100$

$0=0000+1100$

$0: 0 n 00+1100$

$0=0000+1100$

$0.0 n 0 u+1100$

$0: 0 n \theta-\theta+1100$

$0: 0 r 00+1100$

oronod+1100

$0.0 n 00+100$

o. onoutiod

$0=0 n 00+1100$

$0.0100+1100$

$0.0 n 00+1100$

$0.0 n 00+1100$

gonou+1100

0 .

$0.0 n 00+n 00$

0 DONOA $\rightarrow$ HOO

$0=0 n 00+11.00$

$0.0 n 00+n 00$

$0.0 n 00+1100$

$0.0 n 00+1100$
$0 \quad 0 \cos \theta+100$

$0.0000+1100$

$0=0100+1100$

$0 \div 0 n 00+t 100$

$0.0 n 00+1100$

$0: 0 n 00+1100$

$0=0 n 00+1100$

$0.0 n 00+1100$

?

$0=0 n 0 u+1100$

0 onoutiloo

$0.0 n 00+1100$

$0.0 n 00+1100$

$0.0 n 00+100$

$0.0500+1100$

oronogendo

$0: 0 n 00+1100$

:onoptila

$0=0 n 00+1100$

$0=0000+1100$

$0=0$ BOO $+1+00$

$0=0 n 00+1100$

$0.0100+1100$

OOnOO\&HOO

$0.0 n 00 \bullet 1100$

0 ionootil0o

$0=0$ 000+1100

$0.0100+1100$

$0=0100+1100$

ogonout1100

a

$0.0000+1100$

$0=0100+1100$

$0=0 r 0 \theta+1100$

$0.0 n 00+1100$

$0.0 n 0 \theta+1100$
$0.0 n 00+1100$ 
TABLE VIII. Printout of Lower Corner of Delta Response Matrix for Sample Problem

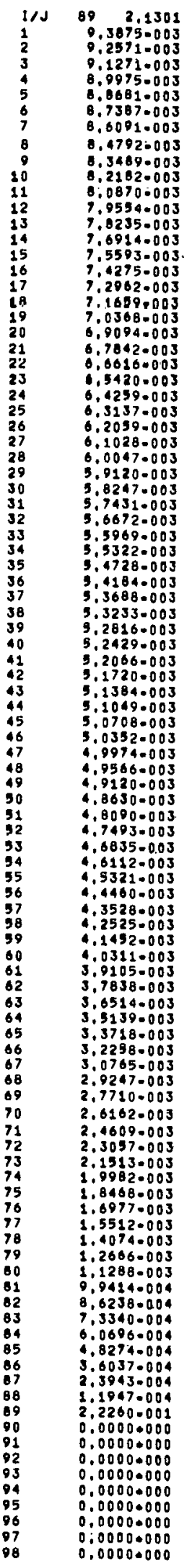

$90 \quad 2.1480$ $9.4020=003$ $9,2729.003$ $9.1456-003$ $9.0115-003$ $8,8819-003$ $8.7525=003$ 8.6232 .003
$8,4930.003$ 8. $3646-003$ $8.2351-003$
$8 ; 1039=003$ $7,9758-003$ 7.8460 .003
$7.762=003$ $7,5867.003$ 7.4578 .003
, $.3202=005$ $7,2016.003$ 7.0733 .003
6.9505 .003 6.8277 .003 6,58040003 6.4747 .003 6.3634 .003 $6.1526 .00 \mathrm{~J}$ 6.0537 .003 $5,9596-003$ $5.0702-003$ $5,7068.003$ $5.6328-003$ $5.5638-003$
$5.4998-003$ 5.4404 .003 $5,3348.003$ $5,2876-003$
$5,2437-003$ 5.2024 .003 5.1253 .003 $5,0512.003$ $5.0139-003$ 4.9740 .003 $4.9339-003$ 4.8428 .003 4.7910 .003
4.7363003 $4,7363.003$ $4.6758=003$ $4.6100-003$ $4,5384.003$ $4,3770-003$ 4.2869 .003 4.0870 .003 $3,9790-003$ $3,8644-003$ $3.7449-003$ $3.6187=003$ $3.3530-003$ 3.2155 .003 $3,0730.003$ 2.9206 .003 $2.7-734-003$ 2.6351 .003
$2.4801=003$ 2:3367.003 $2.1873-003$ 2.0304 .003 1.7437 .003 $1,5986-003$ $1.4553+003$ $1,3140.003$ $1: 0377-003$ $9.0265-004$ 7.6950 .004 $6.3828-004$ $5.0859-004$ $2,5290-004$ $1,2627-000$ $2,1724.001$ $0.0000+000$ $0.0000+000$ $0,0000+000$ $0.0000+000$ $0,0000+000$ $0.0000+000$
$0.0000+000$
$91 \quad 2,1659$ $9.4172-003$ .2854-003 $9.0236-003$ $8,8936=003$ $8,7640=003$ 8.8347 .003 $8,5058.003$ $0.3771-003$ $8,2486=00.3$ $7.9923-003$ 7.06450003 $7,7370=003$ 7.6100 .003
7.4836 .003 $7.4836=003$ .2334 .003 $7,1101=003$
$6,9882=003$ $0.8681-003$
6.75010003 6.5217 .003 6.4118 .003 $6.2024=0.03$ $6.1033-003$ $6.0083-003$ $5.8312=003$ $5,7494=003$ $5.6721-003$
5.5993 .003 $5,5310.003$ $5,4669=003$ $5,3500.003$ $5.2983=003$ 5.2022 .003 $5.1151-003$ $5,0737-003$ $4,9922-003$ 4:0509-003 $4.8086-003$ $4,8181=003$ $4.7689-003$ $4,7164=003$ $4.0600-003$ $4.5342-003$ $4.4640=003$ 4.3887 .003 $4.2219=003$ $4,1302-003$ 3.0350.003 $3,8226-003$ $3,7096-003$ $3,4696-003$ $3.3432-003$ $3,0706.003$ 2., $9432-003$ $2,8043-003$ $2.6634=003$ $2,3209.003$ $2.2329=003$ $2,0881-003$ $1,9798.003$ 1.6548 .003 $1.5116-003$ $1,3693-003$ 1.2280 .003 $1.0878-003$ 8.1085 .004 $6,7304=004$ $5,3795-004$ $4.0274=004$ $2.6815-000$ 2.1189-001 0,00000000 0,00000000 $0.0000+000$ $0.0000+000$ $0.0000+000$ $0,0000+000$ $92 \begin{array}{rr}2 \% 1038 & 93 \quad 2.2017 \\ 9.4307 \div 003 & 9,4436=003\end{array}$ $9=2079-1103$ P:163\%=1103 P.0343-1103 $890136-1103$ $8.6440=1103$ 0 -5151-1103 $823868-1103$ $8.2590-1103$ $8 \pm 1316-103$ $8.0052-1103$ $7.8792-1103$ $7: 753 y-1103$ $7 \div 6295-1103$ $7.5156-1103$ $7-2612-1103$ $7 \geq 24 n y=110.3$ $7=0721-1105$ $629151-1103$ $0.0770-1103$ $0.5664-1103$ $6.4585=1103$ $6.3534=1103$
$6.2515-1103$ $6: 1524-1103$ 6.057101103 5.9661-1103 $5: 7944-1103$ $5: 7144-1103$ $5: 6383=1103$ $5.5661-1103$ $5=4330-1103$ $5: 371 /-1103$ 5. $3131-1103$ $5: 2586-1103$ 5:2n65-1103 $5.118 / 1103$ $5.01700-1103$ $4.9723-103$ $4: 9774-1103$ $4 \div 8830=1103$ $4,7005-1103$ $4.7414-1103$ $4.0900-1103$ $4: 63722-1+03$ $4.5000-1103$ $4.5205-1103$ $43880-1103$ $3164-1103$ $42590-103$ $?: 1585-103$ $39982 \mathrm{~s}-1103$ $3.8851-103$ 3.6804-1103 $3.6801-103$ $3.5711 * 1103$ $33398-1103$ $3: 2184-1103$ $3: 0934-1103$ 2.9653-1103 $2 ! 7 n 0 x-1103$ 04070.1103 $2.2875-1103$ 2! $146 /-1103$

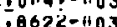
$1 ? 7190-1103$ $135754-1103$ 1: $4310-1103$ .2877-103 $12439-1103$ 8.5670.1104 7. $135 i=1104$ $5=7146-1104$ 2.850U-1104 17404701104 $2=064 b+1101$ 2.0nou+no $0 \pm 0 n 0 u+100$ $0.0 n 00+1100$ $0.0000+1100$ $0.0000+1100$
$0.000 \pi+1100$

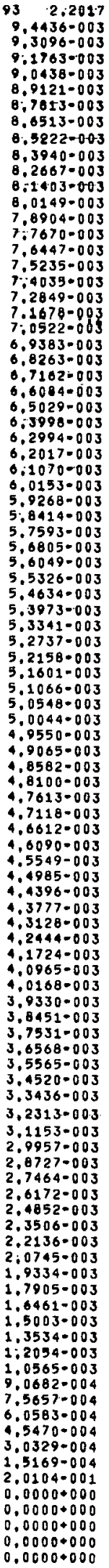

\begin{tabular}{|c|}
\hline 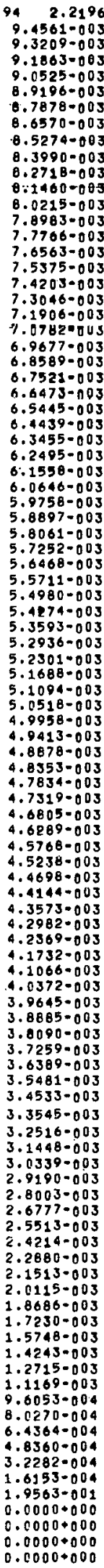 \\
\hline
\end{tabular}

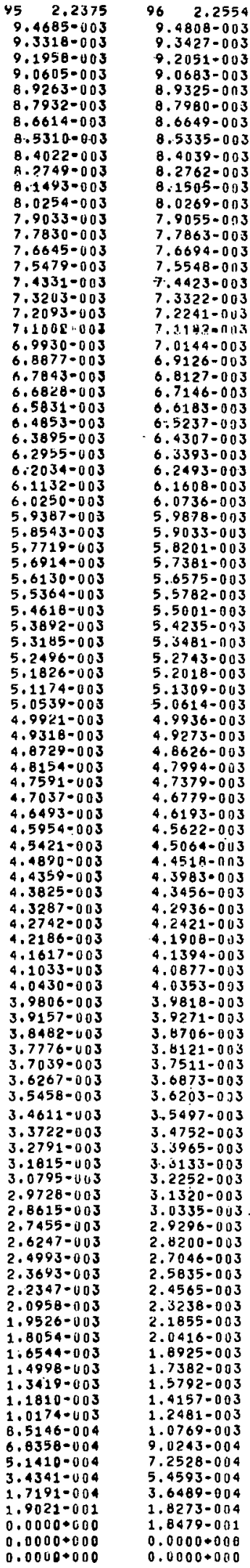




\section{The ITERATE Code}

The program ITERATE had been previously developed for the general treatment of unfolding problems. ${ }^{11}$ Some minor modifications have been introduced to make this program compatible with the WERM and PSNS codes, thereby facilitating operation of the overall system (see Fig. 29). The flow chart in Fig. 33 shows the logical sequence of computations in ITERATE that relate to this system.

The response matrix (that has been generated in WERM and stored on magnetic tape) is read from magnetic tape into ITERATE. An option for printout of this matrix from ITERATE is available and should be compared with the WERM printout (viz. Tables VII and VIII) to ensure proper transfer of the response matrix. The required input data consist of the matrix mesh A, LOWCH, MAXCH, and three vectors. The three input vectors are $\underline{W}$, the output of the detection system; $X 1$, the initial vector for the iteration process; and $\underline{E R}$, the error vector (used in forming the arresting condition). These input vectors and the associated set of midbin energies are listed in the ITERATE output as shown in Table IX. Here we find the $\underline{W}-$ vector (raw proton data) for the test sample problem (where $A=0.0179 \mathrm{MeV}$ per channel, $\mathrm{LOWCH}=31$, and $\mathrm{MAXCH}=128$ ). The choice of a null-error vector creates a zero-arresting bound, and the iteration process continues for a specified total number of iterations. The chosen initial vector for the test problem is the constant vector $X I(I)=1.0, I=1, \ldots, \mathrm{n}$ :

Frintout and punchout of given members of the iteration sequence can be predetermined by fixing input-data parameters. Table X contains, for example, the results of the second iteration with the test problem. Here the $X$ and. WPRIME vectors are just $\underline{X}^{(2)}$ and $\underline{W}^{(2)}$, respectively. The $R$ vector is the residual vector $\underline{R}(n)=\underline{W}-\underline{W}(n)$, where $n=2$ for this case. The norm column is a running sum of the square of the residual-vector components. (Hence, the final value is just the square of the norm of the residual vector.)

Table $X$ also contains the printout of the code for iteration number ten, the final iteration specified for the test problem. Since a null-error, vector was chosen for the test problem, the arresting condition will not stop' the iteration process. It will therefore continue until the maximum allowed number of iterations (specified by input data) has been reached. For this sample problem, the maximum allowed number of iterations was $\mathrm{n}=10$. This was determined by earlier investigations, which revealed rapid convergence and satisfactory solutions. This behavior can also be inferred from a comparison of the magnitudes of the components of the residual vector $R$ for, the second and tenth iterations given in Table $X$. Consequently, the vector $\mathrm{X}^{(10)}$ is: the desired infinite-medium proton spectrum for the sample.problem. 


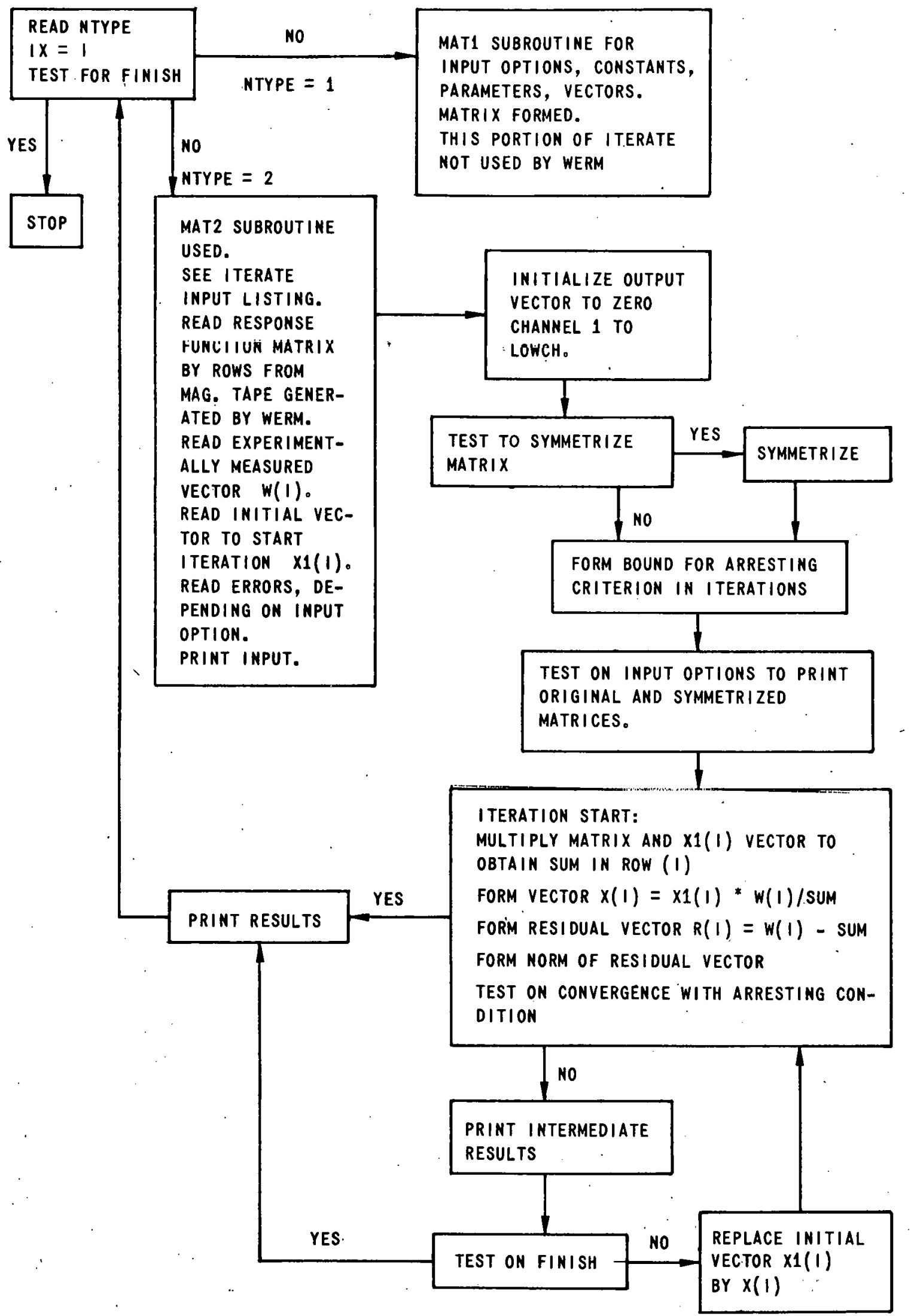

112-9123 Rev. 1

Fig. 33. Flow Chart for Program ITERATE 
TABLE IX. Input Data for Iterative Unfolding of Sample Problem

ITERATIVE GOLUTION OF DETECTION SYSIEM

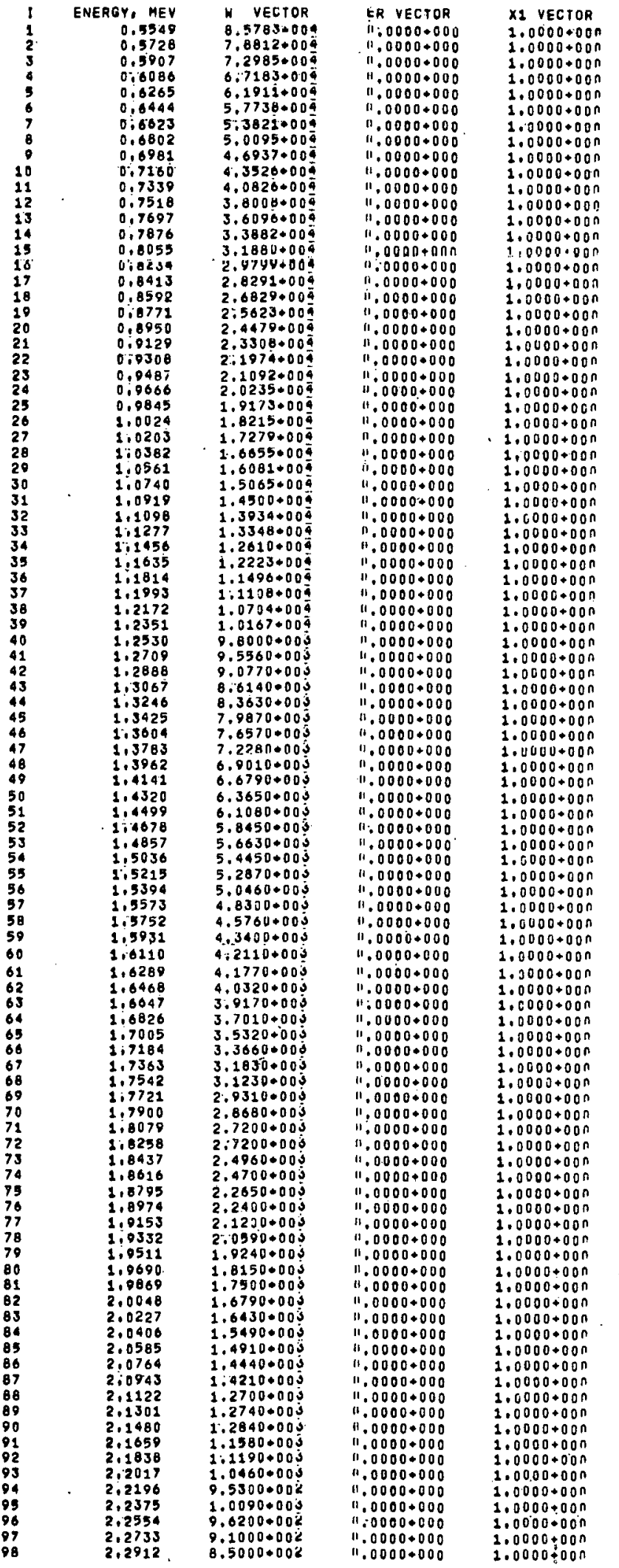




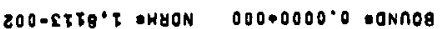

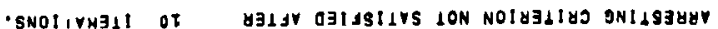

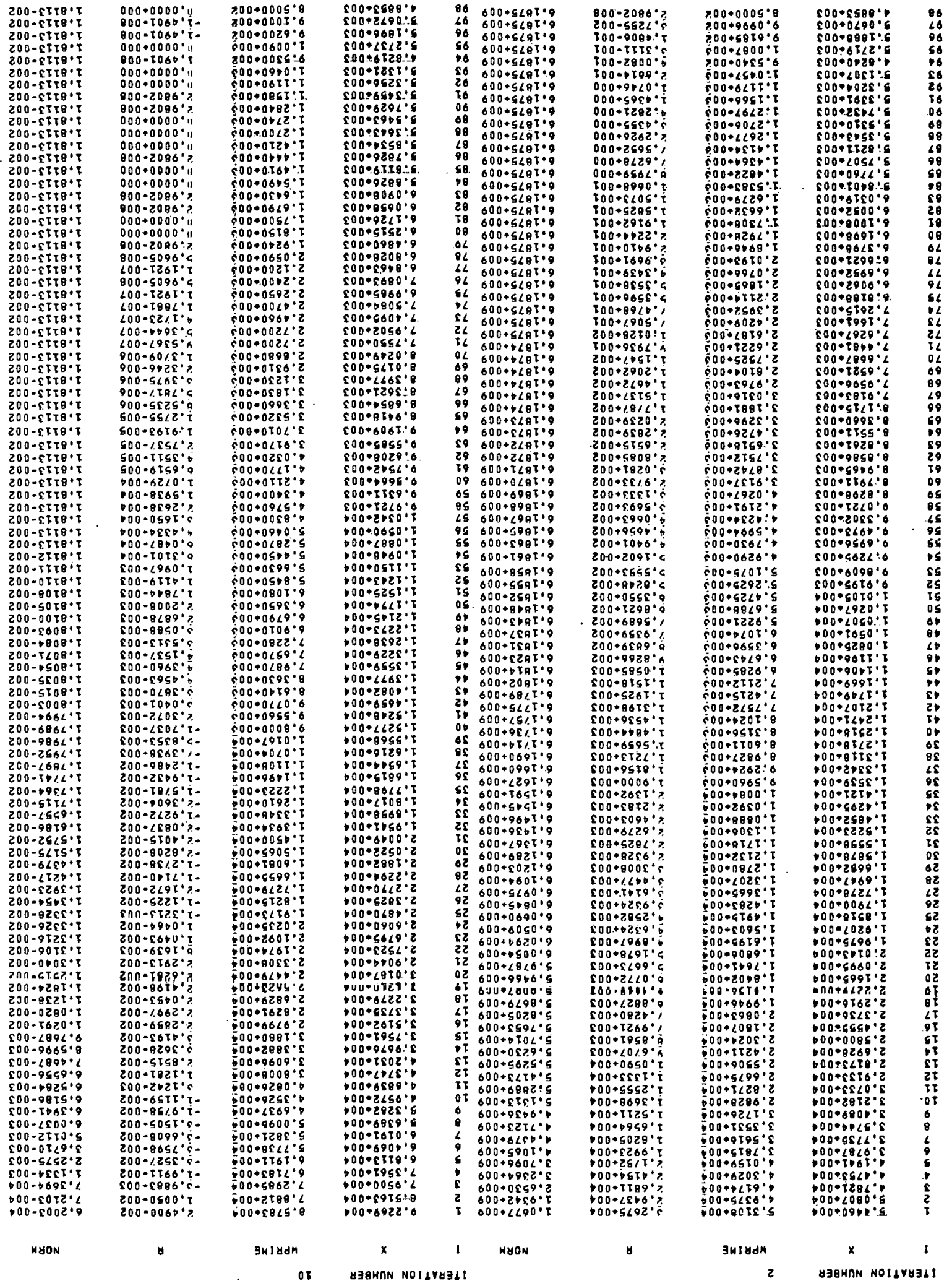

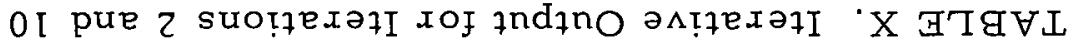


The final iteration vcctor, $x^{(n)}$, is automatically punched out by the ITERATE code for use in the PSNS code. This punchout is obtained whether or not the arresting condition has been satisfied. Consequently, the general applicability of this final vector for the PSNS code must be either predetermined or evaluated from the current ITERATE output. To this end, the code will print out any desired subsequence of results ( Table X) so that convergence properties can be examined. For the sample problem presented here, every second iteration was listed in the ITERATE output.

D. Discussion of PSNS Code and Test Problem

\section{Description of PSNS Code}

Appendix D contains the PSNS (proton spectrum to neutron spectrum) code (CDC-3600 FORTRAN) together with input format and flow diagrams (Figs. 35-38). The code accepts a set of pulse-height distributions measured at various voltages with two counter types and provides a neutron spectrum as output. The need for both a hydrogen and methane-igas filling is justified on grounds of an extended energy response with minimum corrections (see Part III above). A calibration must be available to permit a scale (keV per channel) factor to be assigned to each voltage, and the number of hydrogen atoms present in the effective counting volume of each tube must be known. A multiplicity of voltage settings cannot be avoided without a large reduction in preamplifier noise (a possibility that does not appear to be imminent), if we wish. to measure over the entire energy range. Changes in voltage bring successively overlapping regions of the spectrum within view of the electronics. The chief disadvantage of multiple data sets is an increased time requirement. If adequate neutron-flux levels exist (as will always be the case for reactor measurements) a full (8-hr) day may be necessary for a complete spectrum measurement with good statistics.

The code is straightforward, with the possible exception of the Legendre treatment of response functions; this point was discussed in Section I.D. The WERM code does not provide Legendre coefficients directly; it does provide punched data of the "transformed" response functions from the $\mathrm{N}^{14}(\mathrm{n}, \mathrm{p}) \mathrm{C}^{14}$ pressure-dependent'sequence of measurements. The transformed data are equivalent to the counter response to monoenergetic protons, and simple codes (not presented here) perform the Legendre analysis, and provide least-squares fitting of the Legendre coefficients to obtain a smooth, continuous dependence upon energy.

The Legendre analysis is applied to both the hydrogen and the methane counters, but energy dependence of the Legendre terms is only used for the methane counter. The break region, below which data from the hydrogen counter are used exclusively, is around $100 \mathrm{keV}$. For example, the hydrogen counter used in the test-problem measurement provided here contained 80-psi hydrogen and had a diameter of $1.6 \mathrm{~cm}$. 
It is not difficult to show that protons below $100 \mathrm{keV}$ are subject to negligible wall-and-end effect. It is also important, however, to investigate the effect of the region of the neutron spectrum above the $100-\mathrm{keV}$ limit. This region may contribute a significant number of events, through track truncation, to the spectrum below $100 \mathrm{keV}$. Low equivalent ionizing events can arise from the truncation of very energetic proton recoils, and if these events are significant, the response function for the hydrogen counter would have to be made energy-dependent in the same way as the code allows for the methane counter.

As a numerical test of an upper bound to this effect, an approximate energy-dependent response function for the hydrogen counter was constructed using data derived from the sequence of pressuredependent measurements with the $\mathrm{N}^{14}(\mathrm{n}, \mathrm{p}) \mathrm{C}^{14}$ reaction discussed in Part $\mathrm{I}$. The PSNS test problem (with modification) was run using both the energydependent and an energy-independent response for the hydrogen counter. At $100 \mathrm{keV}$ the difference induced in neutron spectra was $3 \%$, and this difference diminished rapidly as energy decreased. Because of the experimental method used, ${ }^{13}$ the true error below $100 \mathrm{keV}$ is actually less than this calculated bound. In particular, the number of wall-and-end events recorded will be reduced by using the same pulse-shape discrimination intended primarily for the elimination of background gamma radiation.

These observations will certainly not apply other than to neutron spectra comparable to the dilute fast-reactor variety and to counters at least as high in pressure and of comparable size to those considered here. If harder spectra are studied, or if counter stopping power to protons is reduced, these effects would have to be re-examined.

The main features of the PSNS code are discussed in Sections a-f below.

a. Computation of Proton Spectrum. Raw proton spectra (number of counts per kilovolt per.second per $10^{24}$ hydrogen atoms) are derived using the predetermined energy-scale calibration factors for each measurement and the experimental counting time. The hydrogen atom number will ordinarily not be the same for both counters.

b. Integral Response Unfolding. Raw proton spectra (RAPS) are first corrected for response-function effects by a numerical integration according to Eq. 26. Each set of data is corrected starting at the highest-energy set and working downward in energy. There may be an overlap of the energy region for different sets, and to simplify the integration, an energy (MNOLL) for each set is determined and the integration carried out only over the distribution in excess of this energy. The next higher-numbered set is then used to extend the integration to lower energies, terminating finally at the energy point to which the correction is being made. The response function appropriate to each energy in the integration is derived from input amplitudes of the first four Legendre polynomials, 
$\left\{\mathrm{P}_{1}-\mathrm{P}_{4}\right\}$. If the proton-spectrum point being corrected was taken with the methane counter, the Legendre amplitudes are energy-dependent (if energy exceeds ELIMIT). If the point under correction was taken with the hydrogen counter, the Legendre amplitudes are the fixed values CLHYD.

Generally, the response correction, as made here, fails near the upper energy extremity (see Section II.B). The fission-spectrum "tail" in fast reactors extends well beyond the region of measurement; failure of the correction near the limit of measurement will occur regardless of the manner in which a correction is made (iterative unfolding or the integral technique in PSNS).

Ordinarily, an iterative unfolding correction to at least the highest-energy. data set is applied. The integral-response correction will then be skipped in PSNS by setting NUC equal to the number of sets that have been precorrected.

c. Energy Spacing and Neutron-flux Format. Most calculations of neutron spectra involve energy groups chosen to have a fixed fractional, width. Flux per unit lethargy is then the same as group flux. For.this reason, PSNS provides an output flux per unit lethargy at a siequence of edit energies having a fixed fractional spacing; the spacing is input as DINC in the PSNS program.

termined by

d. Slopes and Errors. The slope-taking half interval is de-

$$
\text { Half Interval }=R A+\frac{\dot{R} \cdot B}{\sqrt{\text { energy }}} \text {, }
$$

where RA and RB must be provided as input data to the code. The resolution of a measurement can be related to the slope-taking interval as described in Ref. 14. As energy decreases, statistical effects broaden the intrinsic resolution of these detectors, and the slope-taking interval can be increased correspondingly by making RB nonzero. Each set of responsecorrected data (PRAPS) is tested against the list of preformed edit energies, and if data in the set exist within plus and minus the slope-taking halfinterval around an edit energy, the slope and slope error are determined.

The slope error is assigned on the basis of counting statistics in the data and is determined by using the uncorrected (RAPS) spectrum. If a response correction is made; the corrected proton spectrum (PRAPS) used in the determination of the corrected neutron spectrum will be different from the raw spectrum. The slope error is, consequently, scaled by the factor PRAPS/RAPS to allow for the correction. 
When data are precorrected for response-function effects by ITERATE, the error determined in PSNS will not be the true error; this difference is discussed in Appendix A.

Each set of data is scanned from high to low energy, and measured flux values are assigned to those edit energies contained within the set. Two (or more) sets may be able to contribute to a given edit energy, in which case the weighted average is stored.

If the spacing of edit energies is less than the slope-taking interval, data in common will be used to determine the values of flux and error for adjacent energies. Correlations will occur, and it is not permitted to average fluxes and errors over energy intervals by the simple procedure used for combining counts in a counts-versus-channel number histogram, for example. The mean value of flux is correctly given by the mean value of the individual datum points, over the interval, but the error must be increased (by the square root of the ratio of the slope-taking interval to the spacing interval) over the usual error estimate for the mean. More explicitly, let UE denote the usual rms error for combining quantities $\phi_{i}(i=1,2, \ldots, N)$. If the $\phi_{i}$ are uncorrelated, the mean is given by

$$
\langle\phi\rangle=\frac{1}{N} \sum_{i=1}^{N} \phi_{i},
$$

and the error in the mean becomes

$$
U E=\frac{1}{N} \sqrt{\sum_{\ell=1}^{I N}\left(\operatorname{err} \phi_{i}\right)^{2}},
$$

where $\operatorname{err} \phi_{i}$ is the error in each individual $\phi_{i}$. If the $\phi_{i}$ are neutron fluxes determined by á slope-taking interval $\Delta$ with an energy spacing $\delta$, the mean is the same as above. However, the error in the mean is greater than UE and is given by

$$
\text { True Mean Error }=\sqrt{\Delta / \delta} \cdot \mathrm{UE} \text {. }
$$

If $\Delta$ is less than $\delta$, the $\phi_{i}$ are not correlated and $U E$ is the true error.

These arguments involving errors are important, for example, in a comparison of a measured spectrum with a group calculation. The group-energy width is ordinarily many times the energy spacing $\delta$, and it is usually necessary to average a measurement in order to make a comparison with theory.

e. Heavy-particle Recoils. The correction for carbon and nitrogen recoils is made in PSNS as described in Part III. The fractional 
correction for heavy-particle recoils at each edit energy, HPCOR, is listed in the output. This correction is uncertain for reasons given in Section III.C, and it is convenient to see its magnitude along with the flux and error.

f. Form of Punched Data. Values of the various computed quantities were initialized to zero in the code. If none of the data sets overlap a given edit energy, all quantities will be zero at that energy at the termination of the calculation. This will misrepresent the spectrum, and it is less ambiguous if the code removes these blank locations before printing or punching results.

'L'he energy and the neutron flux and rms flux error are punched for subsequent use as input to special routines for plotting or for comparison with calculation.

\section{2. $\quad$ PSNS Test Problem}

To illustrate the various parameters used in the code, Table XI records a typical fast-reactor spectrum measurement and analysis. PSNS edits all the input material initially. The quantity $\mathrm{C}$, which normally consists of the product of live-time and $\mathrm{H}$-atom number, if absolute fluxes are desired, is given as a relative value for this test problem. The final computed fluxes are, consequently, not absolute.

TABLE XI. Raw Data for First Set of PSNS Test Problem

$$
\begin{aligned}
& \text { Initial Channel }=31 \\
& \text { Final Channel }=128
\end{aligned}
$$

\begin{tabular}{|c|c|c|c|c|c|}
\hline 85783 & 78812 & 72985 & 67183 & 61911 & 57738 \\
\hline 53821 & 50095 & 46937 & 43526 & 40826 & 38008 \\
\hline 36096 & 33882 & 31880 & 29799 & 28291 & 26829 \\
\hline 25623 & 24479 & 23308 & 21974 & 21092 & 20235 \\
\hline 19173 & 18215 & 17279 & 16655 & 16081 & 15065 \\
\hline 14500 & 13934 & 13348 & 12610 & 12223 & 11496 \\
\hline 11108 & 10704 & 10167 & 9800 & 9556 & 9077 \\
\hline 8614 & 8363 & 7987 & 7657. & 7228 & 6901 \\
\hline 6679 & 6365 & 6108 & 5845 & 5663 & 5445 \\
\hline 5287 & 5046 & 4830 & 4576 & 4340 & 4211 \\
\hline 4177 & 4032 & 3917 & 3701 & 3532 & 3366 \\
\hline 3183 & 3123 & 2931 & 2868 & 2720 & 2720 \\
\hline 2496 & 2470 & 2265 & 2240 & 2120 & 2059 \\
\hline 1924 & 1815 & 1750 & 1679 & 1643 & 1549 \\
\hline 1491 & 1444 & 1421 & 1270 & 1274 & 1284 \\
\hline 1158 & 1119 & 1046 & .953 & 1009 & 962 \\
\hline
\end{tabular}


The test spectrum consisted of a 64-channel pulse-height distribution for each set except the first: The first set covered proton energies above gamma background and did not use pulse-shape discrimination. All other sets used pulse-shape discrimination.

The range of usable data started well above channel one. When the lower-energy:regions were measured, pulse-pileup distortion due to saturated ionizing events prevented a clear separation between proton and gamma events as analyzed by the discrimination circuit, unless events at. small values of pulse height were excluded from the spectrum. In addition, pulse-amplitude overload caused some distortion in the last few channels (which were also excluded). These effects tend to worsen as the energy region analyzed diminishes. For example, the lowest-energy spectrum (4300 $\mathrm{V}$ on the hydrogen counter) extended up to only about $2.5 \mathrm{keV}$. Well over $90 \%$ of all the events registered were above this energy, and these events saturated the amplifiers, occasionally by as much as $10^{3}$. The amplifiers used were not especially well suited for counting under these circumstances, and considerably less distortion has been observed with a more recent design.

The input spectra consisted of measured pulse-height distributions, except for the first set, which was precorrected using ITERATE as described in Section II.B. The raw pulse-height distribution for the first set is included just before the results for the test problem (see Table XI). Statistical errors that apply to the ITERATE-corrected distribution are not those supplied by the PSNS code, and an appropriate factor (near unity), described in Appendix $A$, has to be applied. Neutron spectra derived from the ITERATE-corrected first set agree well with spectra derived using the raw pulse-height distribution for the first set in the PSNS code, except near the maximum energy (Fig. 23). The neglect of neutron flux in excess of the upper limit of measurement will introduce a serious systematic error in spectra computed near the limit, regardless of the method used for correcting spectra; this point was discussed in Part II.

The test-problem analysis leads to the same conclusions as those given in Part II. The inclusion of an energy-dependent response function has surprisingly little effect upon the derived neutron spectrum in the region (above $0.5 \mathrm{MeV}$ ) where a considerable distortion of the proton-recoil distribution occurs. This fact is undoubtedly due, in part, to the rapidly falling flux amplitude with increasing energy.

The effect of the non-Gaussian response upon the measurement can be observed by running the test problem (with the raw proton spectrum used in the first set) and then successively omitting orders in Legendre polynomials. The higher-order polynomial terms significantly change the shape of the non-Gaussian response distribution without altering the area under the distribution, which is given by the amplitude of the first polynomial term. The test problem was run with all terms set to zero (ideal counter response), retention of the leading Legendre term, retention of the 


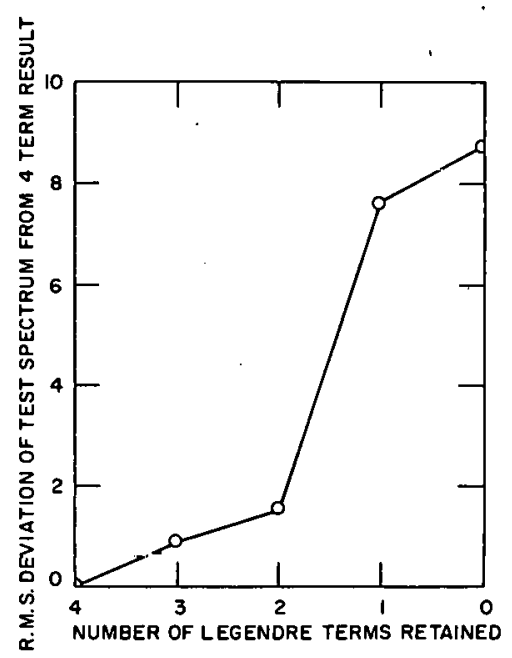

$112-9012$

Fig. 34. Effect of Retention of Legendre Polynomial Terms on Test Spectrum first two terms, retention of the first three terms, and finally with all four terms. The root-meansquare deviation (each spectrum consisted of 147 energy points) of each spectrum from the spectrum retaining all four terms was calculated, and the result is shown in Fig. 34. Retention of the first two terms is sufficient, for this problem, to correct the distribution to one extremely close to the four-term result. Because of the integral nature of the response correction to proton distributions, the perturbation upon neutron spectra caused by the inclusion of the third and fourth terms is quite small.

The code edits the fractional correction that has been made to the neutron flux at each energy due to recoils from carbon and nitrogen $[\operatorname{HPCOR}(\mathrm{K})]$. This correction is zero until an energy $20 \%$ of the maximum measured (see Sec-

tion III.C) has been reached, since the code assumes that the neutron flux vanishes above the measurement region. Below about $500 \mathrm{keV}$, the correction becomes finite (approximately $2 \%$ ). It increases to a maximum of about $6 \%$ near the break region between the methane and hydrogen counters and then drops to less than $2 \%$ as data used in determining the neutron spectrum are derived exclusively from the hydrogen counter. The correction increases with decreasing energy up to a maximum of $10 \%$ at a few keV. (This relatively large (percentage-wise) correction is due to the rapid reduction in neutron flux as energy decreases for the spectrum used as an example.) The significance of this correction made in the code is uncertain at low energies, because of lack of detailed knowledge of $W$ for heavy nuclei. This correction can be reduced by at least half by removing nitrogen from the counter and replacing it by a considerably smaller amount of $\mathrm{He}^{3}$, which can also be used as the calibrating agent. For the test spectrum, a residual 5\% maximum uncertainty in low-energy flux would still remain from carbon recoils. This error is comparable to statistics and quite possibly to other systematic errors existing in the spectrum at low energy. 


\section{APPENDIX A}

Error Estimates for Iterative Unfolding

The elements of the output vector of a given detection system (the measured proton-recoil spectrum for the present system) will possess random statistical error. This fact implies the existence of random error in the iterative solution (i.e., the input vector). Expressions and estimates for this error are desirable. The present treatment will be applicabie only within the context of two-important assumptions. First, we must assume that the iterative unfolding method furnishes appropriate solutions for the given detection system of interest. ${ }^{11}$ If appropriate solutions cannot be found, the method of iterative unfolding tails to delermine the de gircd physical solutions, and for such cases error estimates cannot be regarded as meaningful. Second, it will be assurred that the rcsponse matrix is an exact representation of the systematic behavior of the detection system and therefore does not possess random statistical error. In many instances, this latter assumption is justified since the actual random error in the response matrix is negligible compared with that of the output vector.

Let the matrix representation of the detection system take the form ${ }^{11}$

$$
\underline{Y}=\underline{A} \underline{X},
$$

where $A$ is the response matrix (of order $n$ ) and. $\underline{Y}$ is the output vector. In addition, let $\underline{E}$ represent the random error of the output vector $\underline{Y}$. Hence, we need generally consider systems of the form

$$
\underline{Y}+\underline{E}=\underline{Y}^{\prime}=A \underline{X} .
$$

Under rather broad physical conditions, ${ }^{11}$ application of iterative unfolding for Eqs. A. 1 and A. 2 will lead to the convergent sequences $\{\underline{Y} .(\mathrm{m})\}$ and $\left\{\underline{Y}(m)^{i}\right\}$, respectively. Namely,

$$
\begin{aligned}
& \lim _{m \rightarrow \infty} Y^{(m)}=\underline{Y}, \\
& \lim _{m \rightarrow \infty} \underline{Y}^{(m)}=\underline{Y}^{\prime}=\underline{Y}+\underline{E} .
\end{aligned}
$$

and

It follows from Eqs. A. 3a and A.3b that, for sufficiently large $m, E$ also represents the random error in $\underline{Y}(\mathrm{~m})$. In other words, any given iterate $\underline{Y}(\mathrm{~m})$, for sufficiently large $\mathrm{m}$, possesses the same random statistical behavior attributable to the output vector $\underline{Y}$. As: an example, consider the case in which the output vector $\underline{Y}$. is governed by Poisson statistics. This assumption is commonly used for many particle-detection systems 
and counting experiments that arise in nuclear measurements. For this case, the variance $S_{y_{i}}^{2}=y_{i}, i=1,2, \ldots, n$. In this event, the variance $\mathrm{S}_{\mathrm{y}_{i}}^{2}(\mathrm{~m})$ of $\mathrm{y}_{\mathrm{i}}^{(\mathrm{m})}$ is given by $\mathrm{S}_{\mathrm{y}_{i}}^{2}(\mathrm{~m})=\mathrm{y}_{i}^{(\mathrm{m})}=\mathrm{S}_{\mathrm{y}_{\mathrm{i}}}^{2}=\mathrm{y}_{\mathrm{i}}, \mathrm{i}=1,2, \ldots, \mathrm{n}$, for sufficiently large $\mathrm{m}$.

The variance $S_{x_{i}}^{2}(m)$ of any given element $x_{i}^{(m)}$, in the iterative solution $\underline{x}^{(m)}$, can be calculated directly from the matrix relation

$$
\underline{Y}^{(\mathrm{m})}=\underline{A X}^{(\mathrm{m})} \text {. }
$$

We find

$$
S_{x_{i}}^{2}(m)=\sum_{j=1}^{n}\left[\left(a^{-1}\right)_{i j}\right]^{2} \cdot S_{y_{j}}^{2}(m), \quad i=1,2, \ldots, n
$$

and therefore, for sufficiently large $\mathrm{m}$,

$$
s_{x_{i}}^{2}(m)=\sum_{j=1}^{n} \cdot\left[\left(a^{-1}\right)_{i j}\right]^{2} S_{y_{j}}^{2}, \quad i=1,2, \ldots, n,
$$

where $\left(a^{-1}\right)_{i j}$ are the elements of the inverse matrix, $A^{-1}$. For Poisson statistics, Eqs. A. 5 a and A. 5b become

$$
S_{x_{i}}^{2}(m)=\sum_{j=1}^{n}\left[\left(a^{-1}\right)_{i j}\right]^{2} y_{j}^{(m)}, \quad i=1,2, \ldots, n
$$

and

$$
S_{x_{i}}^{2}(m)=\sum_{j=1}^{n}\left[\left(a^{-i}\right)_{i j}\right]^{2} y_{j}, \quad i=1,2, \ldots, n .
$$

These expressions are cumbersome and difficult to use since they involve elements of the inverse matrix, $\mathrm{A}^{-1}$. Consequently, a less precise but more useful result, not involving $\mathrm{A}^{-1}$, will be developed below. This analysis is based on the observation that most response matrices possess dominant diagonal elements and relatively small off-diagonal elements. Moreover, for diagonal response matrices, it follows from Eqs. A.4 and A.5a that the relative error in $\underline{X}^{(m)}$ is identical with that of $\underline{Y}^{(m)}$; hence it is identical with that of $\underline{Y}$. Therefore, we may qualitatively infer for dominantly diagonal response matrices that the relative error in $\underline{X}(m)$ is approximately that of $\underline{Y}$. 
To establish this conjecture rigorously, let the matrix A be partitioned in the form

$$
A=A_{d}+A_{0}
$$

where the matrices $A_{d}$ and $A_{0}$ are defined as

$$
\left.\begin{array}{ll}
\left(A_{d}\right)_{i i}=a_{i j}, & i=1,2, \ldots, n, \\
\left(A_{0}\right)_{i i}=\left(a_{0}\right)_{i i}=0, & i=1,2, \ldots, n, \\
\left(A_{0}\right)_{i j}=\left(a_{0}\right)_{i j}=a_{i j}, \quad i \neq j, \quad 1, j=1,2, \ldots, i i .
\end{array}\right\}
$$

Consequently, $A_{d}$ is simply a diagonal matrix whose elements coincide with the diagonal elements of $A$, and the matrix $A_{0}$ contains the remaining off-diagonal elements of A.

Taking differentials in Eq. A.4 and using Eq. A.7, we obtain

$$
\underline{\underline{Y}}=\left(\mathrm{A}_{\mathrm{d}}+\mathrm{A}_{0}\right) \underline{\delta X} .
$$

Since it has been assumed that $m$ is sufficiently large to have established convergent results, the superscript $\mathrm{m}$ of the vectors $\underline{X}^{(\mathrm{m})}$ ) and $\underline{Y}(\mathrm{~m})$ has been omitted. In Eq. A.9, the differential vector $\delta Y$ can be identified with the random error vector $E$; i.e., $\delta y_{i}=e_{i}, i=1, \overline{2}, \ldots, n$. Let us form the ensemble average $\left\langle\delta y_{i}: \delta y_{i+1}\right\rangle$, using the representation given in Eq. A. 9 . The quantities $\delta y_{i}$ and $\delta y_{i+1}$ are independent random variables. We there fore have

$$
\begin{aligned}
& \left\langle\delta y_{i} \cdot \delta y_{i+1}\right\rangle=\left\langle[ a _ { i i } \delta x _ { i } + \sum _ { j = 1 } ^ { n } ( a _ { 0 } ) _ { i j } \delta x _ { j } ] \left[ a_{i+1} i+1=x_{i+1}\right.\right. \\
& \left.+\sum_{k=1}^{n}\left(a_{0}\right)_{i+1 k} \delta x_{k}\right] j=0
\end{aligned}
$$

which leads to

$$
\left\langle\delta x_{i} \cdot \delta x_{i+1}\right\rangle=\frac{-i}{a_{i i} a_{i+1} i+1} \sum_{j, k=1}^{n}\left(a_{0}\right)_{i j}\left(a_{0}\right)_{i+1 k}\left\langle\delta x_{j} \delta x_{k}\right\rangle . \quad(A * 10 b)
$$

Since the off-diagonal elements are no larger than $\mathrm{n}^{-1}$, the righthand side of Eq. A. $10 \mathrm{~b}$ will become negligible for sufficiently large. n. 
This conclusion is based on physical conditions implied by the matrix representation of the detection system. Namely, the physical description implies that the correlation between any two elements $x_{i}$ and $x_{\ell}$ decreases as $|i-l|$ increases. It follows that the correlation between $\delta x_{i}$ and $\delta x_{l}$ is an even more rapidly decreasing function of $|i-l|$. We may therefore assume that, for sufficiently large $n$, the quantities $\left\{\delta x_{i}\right\}$ are independent random variables.

The validity of this assumption can also be demonstrated by using the relation

$$
\delta x_{i}=\sum_{i=j}^{n}\left(a^{-1}\right)_{i j} \delta y_{j}
$$

to obtain a direct comparison of $\left\langle\left(\delta x_{i}\right)^{2}\right\rangle$ and $\left\langle\delta x_{i} \cdot \delta x_{l}\right\rangle$. We find

$$
\left\langle\left(\delta x_{i}\right)^{2}\right\rangle=\sum_{i=j}^{n}\left[\left(a^{-1}\right)_{i j}\right]_{;-\infty}^{2}\left\langle\left(\delta y_{. j}\right)^{2}\right\rangle
$$

and

$$
\left\langle\delta x_{i} \cdot \delta \dot{x}_{l}\right\rangle=\sum_{j=1}^{n}\left(a^{-i}\right)_{i j}\left(a^{-1}\right)_{\ell, j}\left\langle\left(\delta y_{j}\right)^{2}\right\rangle
$$

Introducing appropriate mean values, $\mathrm{m}_{\mathrm{d}}^{2}$ and $\mathrm{m}_{0}^{2}$, for the set of variances $\left\langle\left(\delta \mathrm{y}_{\mathrm{i}}\right)^{2}\right\rangle$ that appear in these expressions, we have

$$
\left\langle\left(\delta \mathrm{x}_{\mathrm{i}}\right)^{2}\right\rangle=\mathrm{m}_{\mathrm{d}}^{2} \sum_{\mathrm{j}=1}^{\mathrm{n}}\left[\left(\mathrm{a}^{-1}\right)_{\mathrm{ij}}\right]^{2}
$$

and

$$
\left\langle\delta x_{i} \cdot \delta x_{\ell}\right\rangle=m_{0}^{2} \sum_{j=1}^{n}\left(a^{-1}\right)_{i j}\left(a^{-1}\right)_{\ell j}
$$

The resulting summations, which appear in Eqs. A.13a and A. 13b, now have a simple interpretation. In particular, these sums represent elements of the matrix $A^{-1} \cdot \tilde{A}^{-1}=(\tilde{A} \dot{A})^{-1}$. Consequently we can write

$$
\left\langle\left(\delta \mathrm{x}_{\mathrm{i}}\right)^{2}\right\rangle=\mathrm{m}_{\mathrm{d}}^{2}\left[(\tilde{\mathrm{A}} \mathrm{A})^{-1}\right]_{\mathrm{ii}}
$$


and

$$
\left\langle\delta x_{i} \cdot \delta x_{\ell}\right\rangle=m_{0}^{2}\left[(\tilde{A} A)^{-1}\right]_{i \ell}
$$

It follows from Eqs. A. $14 a$ and A. 14b that $\left.\left\langle\left(\delta x_{i}\right)^{2}\right\rangle\right\rangle\left|\left\langle\delta x_{i} \cdot x_{\ell}\right\rangle\right|$ for dominantly diagonal response matrices. Moreover, Eq. A. 14b establishes the nature of the rapid decrease in $\left\langle\delta x_{i} \cdot \delta x_{\ell}\right\rangle$ with increasing $|i-l|$. Thus, when $A$ is dominantly diagonal, so is $A^{-1}$.. Furthermore, for A dominantly diagonal, ÂA will be even more dominantly diagonal. Consequently, as we move away from the dominant diagonal, the off-diagonal elements of $(\mathrm{AA})^{-1}$ can be expected to decrease rapidly:

Calculation of the variance of $y_{i}$ can be significantly simplified by using the approximate independence of the $\left\{\delta x_{i}\right\}$. Manipulation of Eq. A. 9 yields

$$
\left\langle\left(\delta y_{i}\right)^{2}\right\rangle=\left(a_{i i}\right)^{2}\left\langle\left(\delta x_{i}\right)^{2}\right\rangle+\sum_{j=1}^{n}\left[\left(a_{0}\right)_{i j}\right]^{2}\left\langle\left(\delta x_{j}\right)^{2}\right\rangle
$$

Using once more the fact that the off-diagonal matrix elements, $\left(a_{0}\right)_{i j}$, vanish at least as rapidly as $\mathrm{n}^{-1}$, Eq. A. 15 reduces to the approximate condition

$$
\left\langle\left(\delta y_{i}\right)^{2}\right\rangle \stackrel{\cong}{\cong} a_{i i}^{2}\left\langle\left(\delta x_{i}\right)^{2}\right\rangle
$$

for sufficiently large $n$. We can therefore wite.

$$
\frac{\left\langle\left(\delta x_{i}\right)^{2}\right\rangle^{1 / 2}}{x_{i}} \cong \frac{y_{i}}{a_{i i} x_{i}} \cdot \frac{\left\langle\left(\delta y_{i}\right)^{2}\right\rangle^{1 / 2}}{y_{i}}
$$

Hence, for dominantly diagonal response matrices of sufficiently large order, the relative error in an element $x_{i} \in \underline{X}$ is approximately equal to the relative error in the corresponding element $y_{i} \in \underline{Y}$. For Poisson statistics, Eq. A. 17 reduces to

$$
\left\langle\left(\delta \mathrm{x}_{\mathrm{i}}\right)^{2}\right\rangle^{1 / 2} \cong \frac{\left(\mathrm{y}_{\mathrm{i}}\right)^{1 / 2}}{\mathrm{a}_{\mathrm{i} i}}
$$

Some mention can be made of relaxing the condition that the response matrix be dominantly diagonal. To establish the (approximate) independence of the random variables $\left\{\delta x_{i}\right\}$, this condition may be too conservative for many applications. Since the inverse matrix elements, $\left(a^{-1}\right)_{i j}$, can be positive or negative, we can generally anticipate a great deal of cancellation in the sum of Eq. A. $13 \mathrm{~b}$ for sufficiently large n. Consequently, the condition 


$$
\left\langle\left(\delta \mathrm{x}_{\mathrm{i}}\right)^{2}\right\rangle>>\left|\left\langle\delta \mathrm{x}_{\mathrm{i}} \delta \mathrm{x}_{\ell}\right\rangle\right|
$$

may actually hold in many applications under less restrictive assumptions. In this event, Eq. A. 15 provides the basis for a matrix representation of an unfolding problem for the error estimates. Namely

$$
\underline{S}_{y}=B \underline{S}_{x},
$$

where the elements of $\underline{S}_{y}$ and $\underline{S}_{\mathbf{x}}$ are the variances

$$
\left\{\left\langle\left(\delta y_{i}\right)^{2}\right)\right\} \text {, and }\left\{\left\langle\left(\delta x_{i}\right)^{2}\right\rangle\right\} \text {, }
$$

respectively. The matrix elements $b_{i j}$ of $B$ are defined by

$$
b_{i j}=\left(a_{i j}\right)^{2}, \quad i, j=1,2, \ldots, n \text {. }
$$

This new unfolding problem is an analog of the original unfolding problem. Moreover, iterative unfolding is directly applicable since all pertinent physical conditions are obviously met. ${ }^{11}$ The essential feature of this error-estimate unfolding problem is that the new response matrix elements are merely the square of the elements in the original response matrix (cf. Eq. A.20).* For such cases, iterative unfolding can be used, in principle, to determine error estimates, and thereby we may again avoid the problems associated with both calculating and using inverse matrix elements.

\footnotetext{
* Here, the applicability of Poisson statistics would imply an even closer analogy, since it follows that $\underline{S}_{\mathrm{y}}=\underline{\mathrm{Y}}$ for Poisson statistics.
} 
APPENDIX B

WERM Code Listing

TABLE B.I. WERM Input

\begin{tabular}{|c|c|c|c|}
\hline Card & Variable & Format & Description \\
\hline $1-4$ & $E$ & $12 \mathrm{~F} 6.4$ & Energy table. \\
\hline $5-8$ & RGE & $12 \mathrm{~F} 6.4$ & Corresponding proton range table. \\
\hline \multirow[t]{12}{*}{9} & AMEV & E1.2.4 & MeV per channel (matrix mesh). \\
\hline & PN & E12.4 & Molecular density for spectral measurement. \\
\hline & TFACT & El2.4 & Temperature corrected to $25.5^{\circ} \mathrm{C}$. \\
\hline & LOWCH & I6 & Starting channel for matrix mesh. \\
\hline & MAXSH & 16 & Ending channel for matrix mesh. \\
\hline & NTRI & 13 & $\begin{array}{l}0=\text { no triangularization of matrix. } \\
\frac{t}{=} \text { triangularize. }\end{array}$ \\
\hline & NDEL & I3 & \pm defines $R(J, J)$ as normalized Gaussian area. \\
\hline & NPNP & 13 & $\begin{aligned}-= & \text { magnetic-tape write of matrix. } \\
0= & \text { matrix print and test on NTEST to punch. } \\
& (\text { See NTEST.) } \\
+= & \text { Test on NTEST. }\end{aligned}$ \\
\hline & NCOMB & I3 & $\begin{aligned} 0= & \text { combined fit of Gaussian and non-Gaussian. } \\
-,+= & \text { Gaussian only. No more than } 60 \text { data } \\
& \text { channels. }\end{aligned}$ \\
\hline & NPUNCH & 13 & $\begin{aligned} t_{1}- & =\text { no punch of intermediate results. } \\
0 & =\text { punch of intermediate results. }\end{aligned}$ \\
\hline & NTRAN & I3 &,$-+=$ do not print data transformation factors. \\
\hline & NTEST & 13 & $\begin{aligned}+ & =\text { punch parameters for interpolated re } \\
& \text { sponse function (NF modulo NTEST) } \\
-, 0= & \text { no punch. }\end{aligned}$ \\
\hline & NVMM & 13 & $-,+=X_{1}, X 2, X 3$ are read in and VMM is bypassed. \\
\hline & NP & 13 & $t_{,-}=$no printout of coefficients and parameters. \\
\hline & NPOT & . I3 & $\begin{aligned} t_{,-}= & \text {no punch of relative Gaussian area and } \\
& \text { cosine coefficient for LSQPOL on DDP }-24 \\
& \text { computer. } \\
0= & \text { punch. }\end{aligned}$ \\
\hline 10 & Run Identification & $20 \mathrm{~A} 4$ & \\
\hline \multirow[t]{4}{*}{11} & PNPRYM & E12.4 & $\begin{array}{l}\text { Molecular density for each pressure-response } \\
\text { measurement. }\end{array}$ \\
\hline & $\operatorname{EOP}(\mathrm{NC})$ & F6.0 & Estimate of Peak channel. \\
\hline & CRES & F6.0 & Resolution of Gaussian. \\
\hline & IV & 16 & $\begin{array}{l}\text { Number of channels down from peak to start of } \\
\text { test for half-height. }\end{array}$ \\
\hline $12-24$ & Data for first run & $\mathrm{I} 0 \mathrm{~F} 8.0$ & Highest pressure is input first. \\
\hline $25 \ldots$ & Repeat of Cards $10-24$ & & No more than 30 pressure runs may be used. \\
\hline
\end{tabular}


TABLE B.JT. Other Variables Used in WERM

\begin{tabular}{|c|c|}
\hline Variable & Description \\
\hline NC & Number of individual pressure-response measurements, $\leq 30$ \\
\hline EOCO & $\begin{array}{l}\text { (0.615 MeV/estimate of Gaussian peak channel.) Used to generate } \\
\text { ionization equivalent energy scale. }\end{array}$ \\
\hline $\operatorname{ERG}(\mathrm{I})$ & $\begin{array}{l}\text { Energy (MeV) scale formed by EOCO * I - } 0.03075, I=1,128 \text {. } \\
\text { Subtraction of } \mathrm{C}^{14} \text { recoil energy. }\end{array}$ \\
\hline RE:LG(NC) & $\begin{array}{l}\text { Relative Gaussian area formed from peak and width at half-height } \\
\text { fitted by VMM subroutine. }\end{array}$ \\
\hline $\operatorname{DIF}(\mathrm{I})$ & $\begin{array}{l}\text { Differences formed by subtracting fitted and extrapolated Gaussian } \\
\text { from data (I). }\end{array}$ \\
\hline $\operatorname{ERGP}(\mathrm{I})$ & Transformed energy. \\
\hline $\mathrm{EOP}(\mathrm{NC})$ & $\begin{array}{l}\text { Center position of Gaussian peak in transformed energy for } \\
\text { individual response measurement. }\end{array}$ \\
\hline $\mathrm{X} 1(2, \mathrm{NC})$ & $\begin{array}{l}\text { Width at half-height of Gaússian in transformed energy for } \\
\text { individual response measurement. }\end{array}$ \\
\hline $\operatorname{EPl}(\mathrm{Gl}$ or $\mathrm{G} 2)$ & $\begin{array}{l}\text { Polynomial representation of } \epsilon \text { for data transformation with } \\
\text { energy } \leq 0.4 \mathrm{MeV} \text {. }\end{array}$ \\
\hline $\operatorname{EP} 2(G 1$ or $G 2)$ & $\epsilon$-function used in data transformation for energies $>0.4 \mathrm{MeV}$. \\
\hline TRANS(I) & Ratio of transformed data to original data. (Transformation factor.) \\
\hline $\mathrm{AN}(\mathrm{NC}, \mathrm{I})$ & $\begin{array}{l}\text { Fourier constants formed in non-Gaussian region for analytical } \\
\text { function evaluation. }\end{array}$ \\
\hline $\operatorname{EOF}(\mathrm{I})$ & $\begin{array}{l}\text { Response function matrix energy scale formed from LOWCH to } \\
\text { MAXCH times AMEV (midbin energies). }\end{array}$ \\
\hline $\operatorname{XIM}(2, I)$ & Width of Gaussian at half-height for matrix formulation. \\
\hline $\operatorname{FITG}(\mathrm{NC})$ & Least-squares polynomial-fitted RELG(I). \\
\hline $\mathrm{XI}(1, \mathrm{NC})$ & Peak of Gaussian formed from FITG(I) and fitted XI(2,NC). \\
\hline COSAREA(NC) & Integral of non-Gaussian area. \\
\hline $\mathrm{RN}(\mathrm{NC})$ & Normalization factor formed from COSAREA and FITG for AN's. \\
\hline $\operatorname{FITGM}(\mathrm{I})$ & Fitted relative Gaussian area for matrix formulation. \\
\hline $\operatorname{XIM}(1, I)$ & Corresponding Gaussian peak for matrix usage. \\
\hline $\operatorname{ANM}(I, J)$ & Fourier constants for matrix. \\
\hline $\mathrm{SC}(\mathrm{I})$ & Scale factor formed from fitted normalized AN's to renormalize same. \\
\hline$R(I, J)$ & Response matrix elements. \\
\hline
\end{tabular}


1. Printout of Program WERM

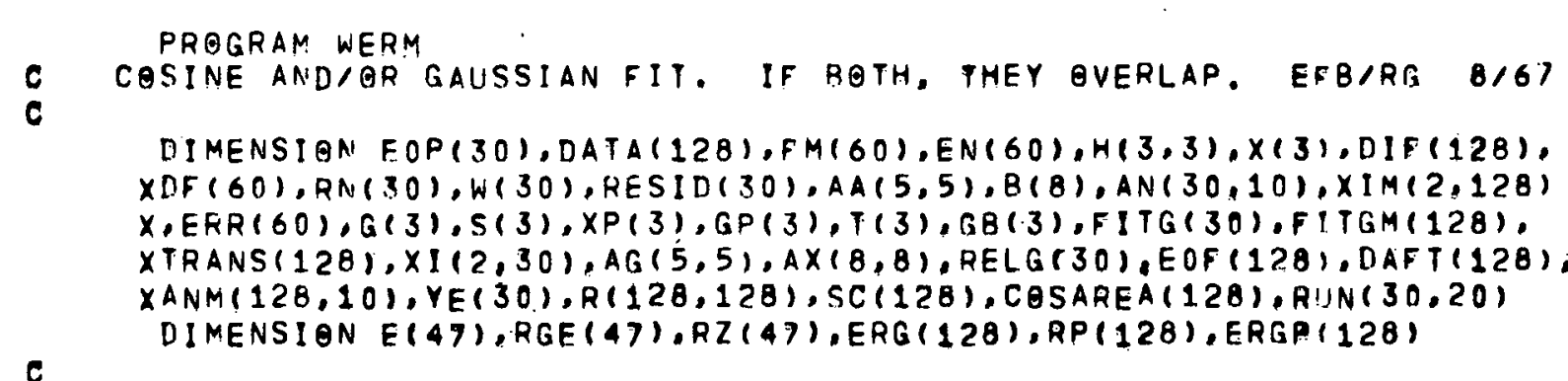

$c$

COMMON H,X,G,S,XP,GP,T,GB,M,N,L,LS,MI,MS,NS, IT,GS,GSP,GTP,GSS,GTT, $X G S B, F, F P, F A, E P, K, P, F O, R S, S L, Z, O, A, E L, D E L T A, F B$

6

COMMON/XYZ/EN,FM,ERR,NG, DF

c

COMMON/12345/EOF, R, ANM, NF, XIM,AN,XI,EOP, AMEV,HALFA,LC,NTRI, NP, NC, XFITG, COSAREA, SC, NDEL, SUM, RELG, ERG,ERGP, TRANS, NTRAN,B, JA, NVMM

c

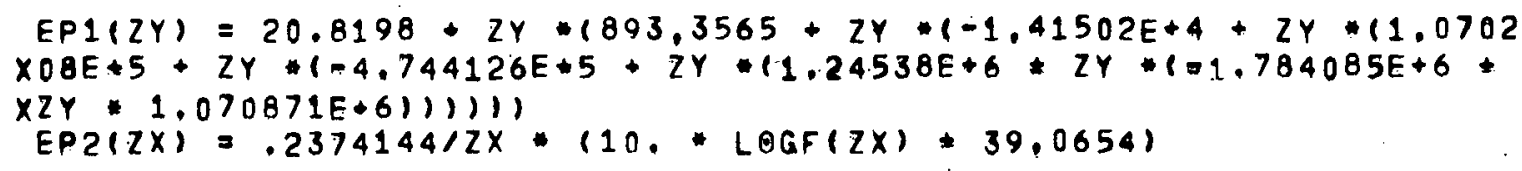




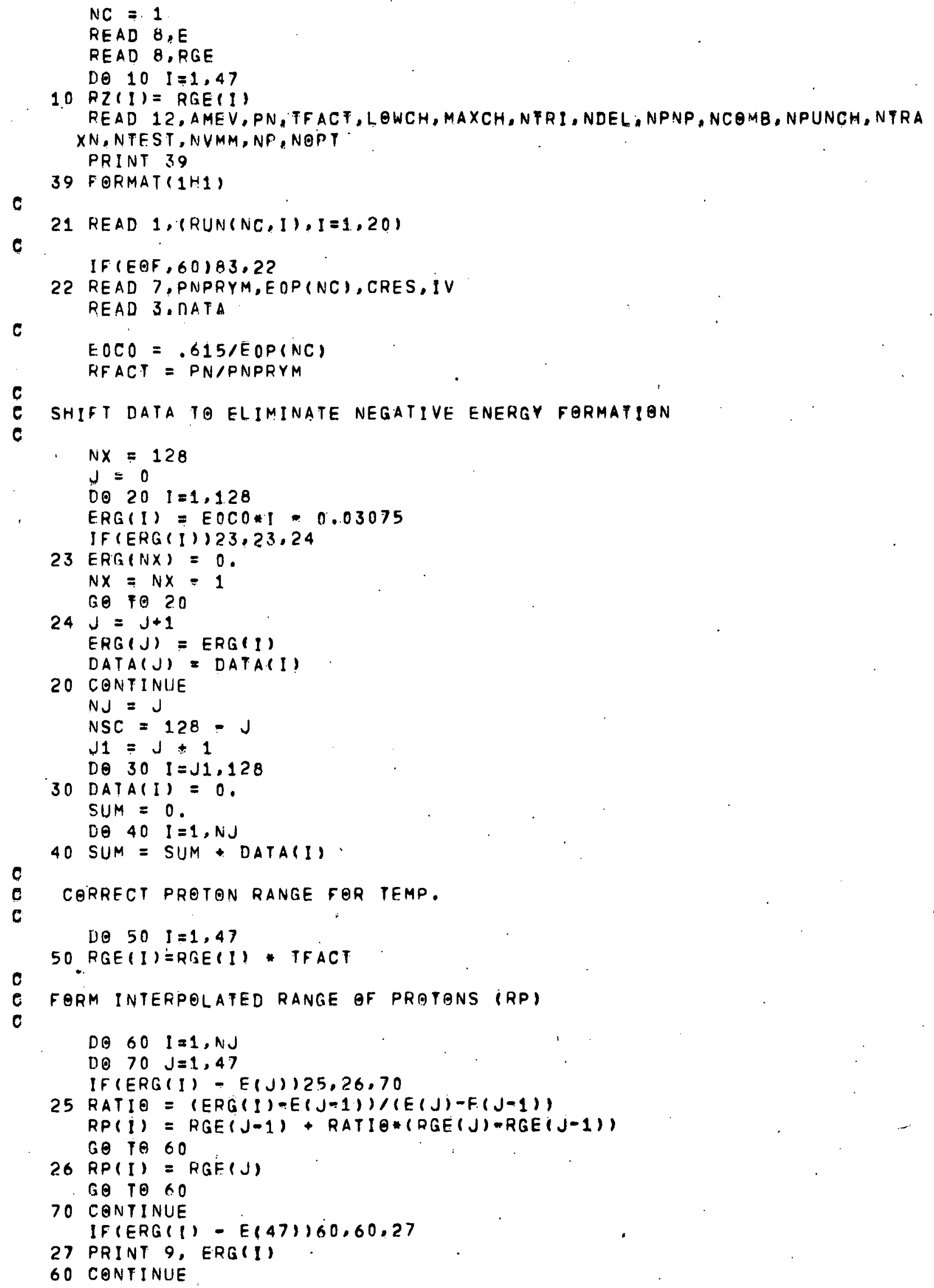




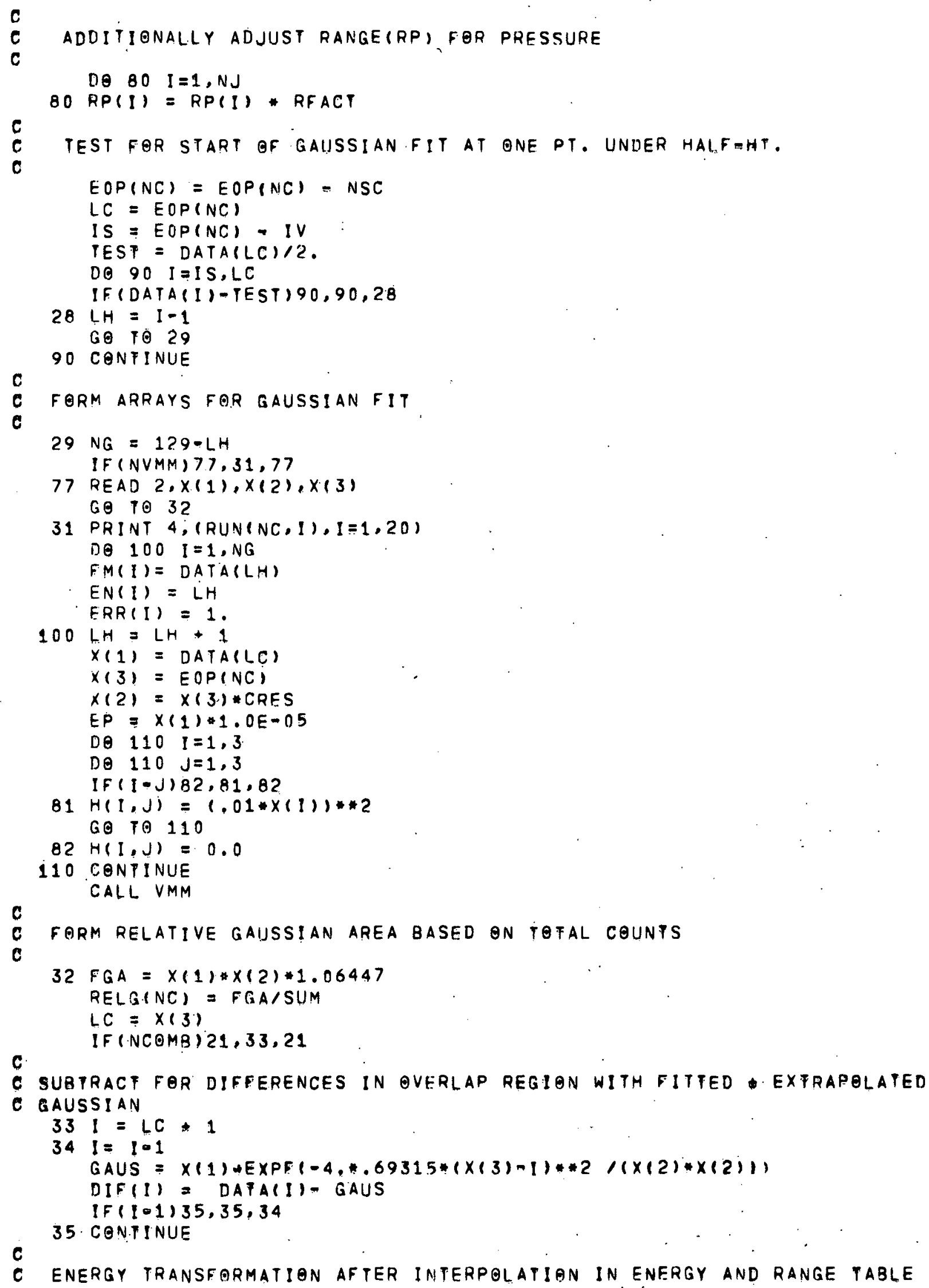




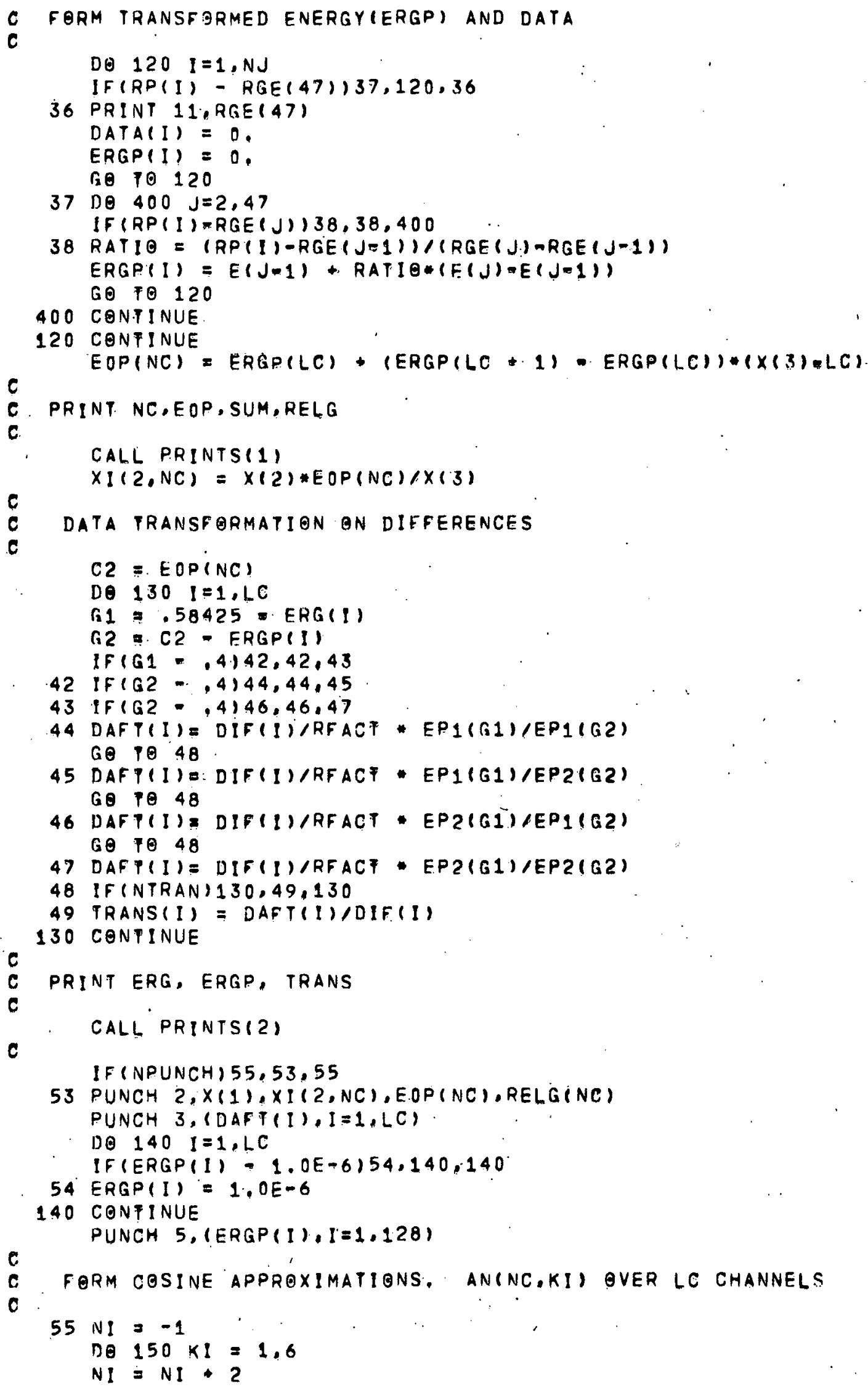




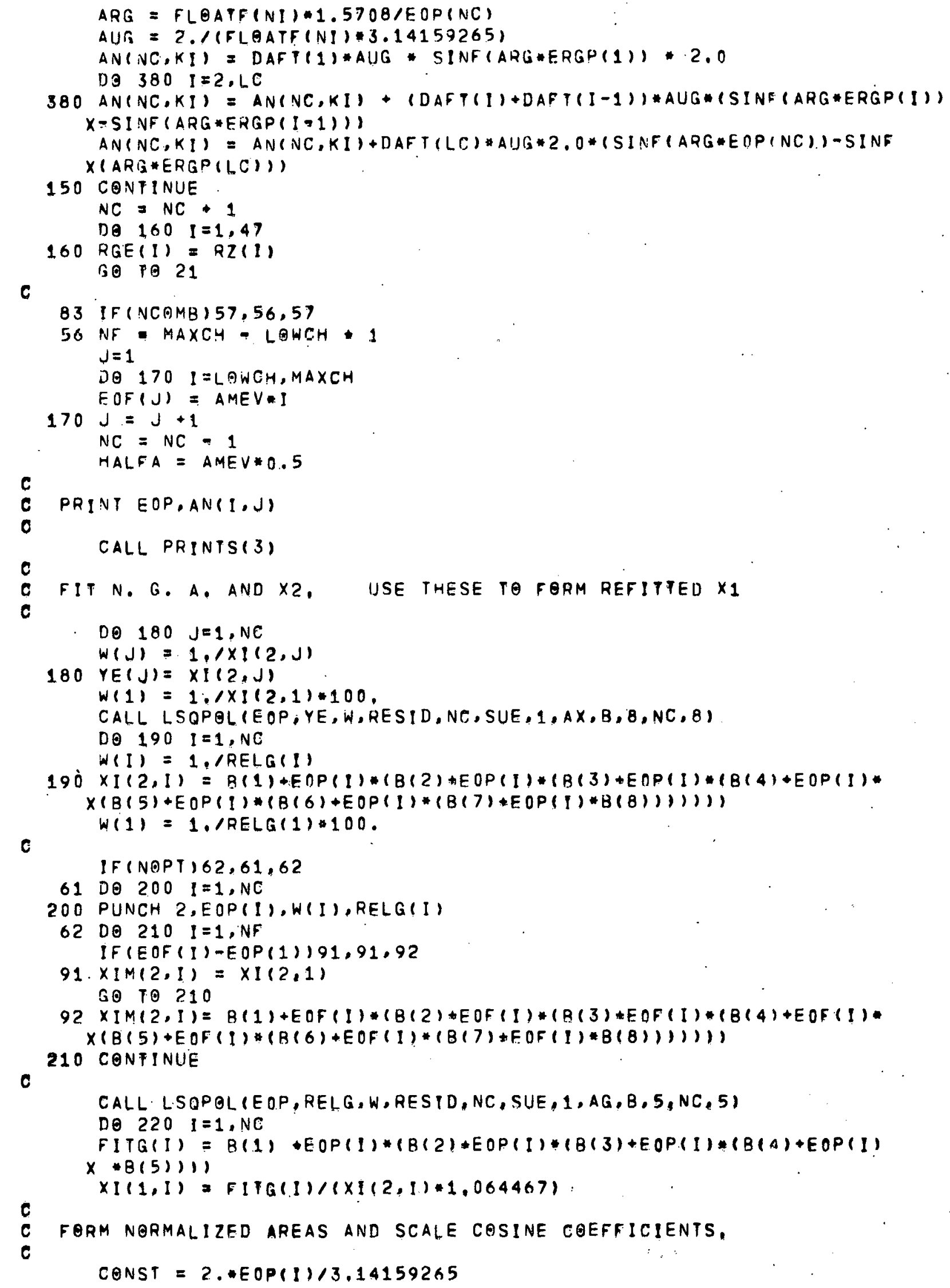




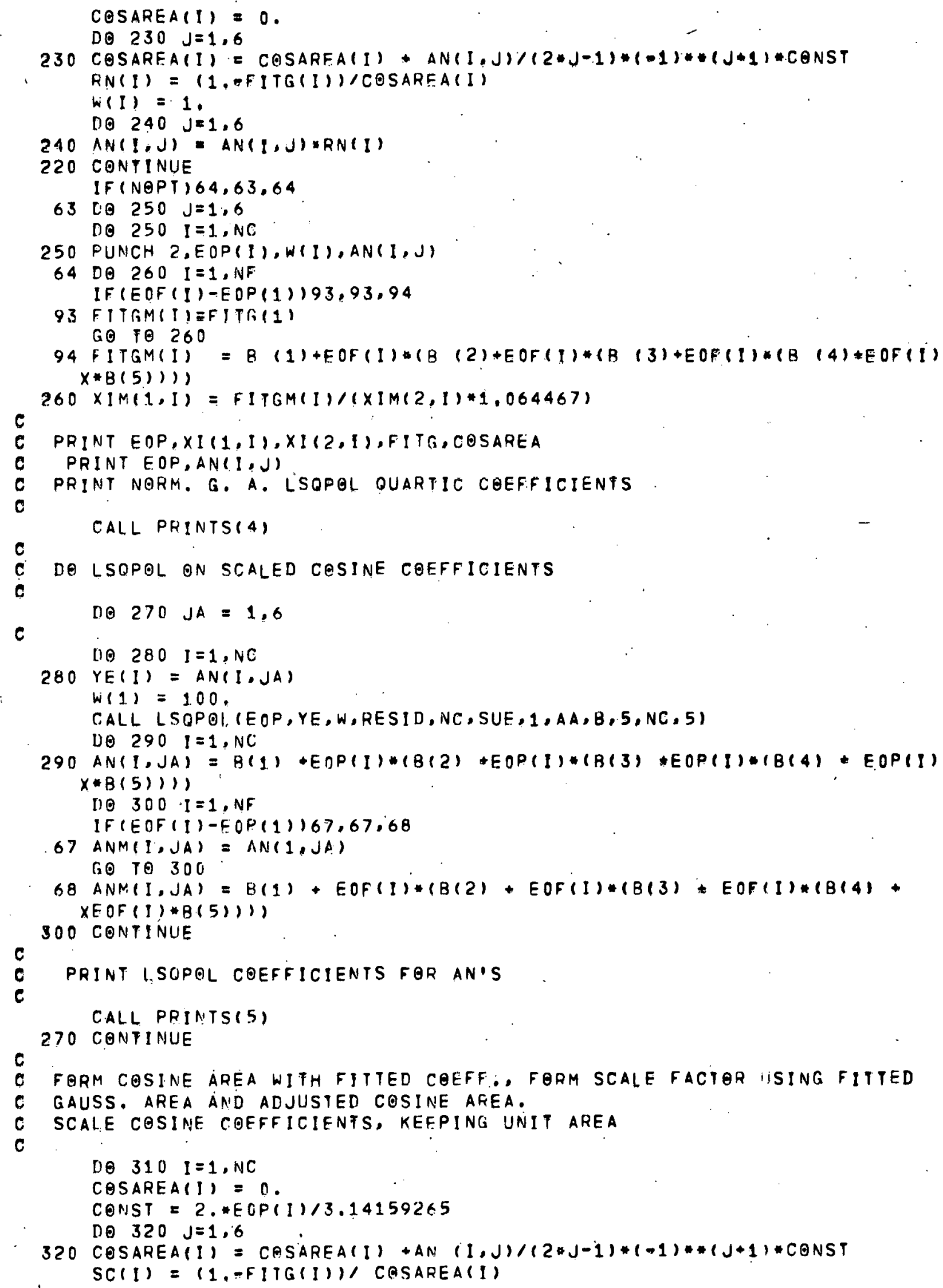




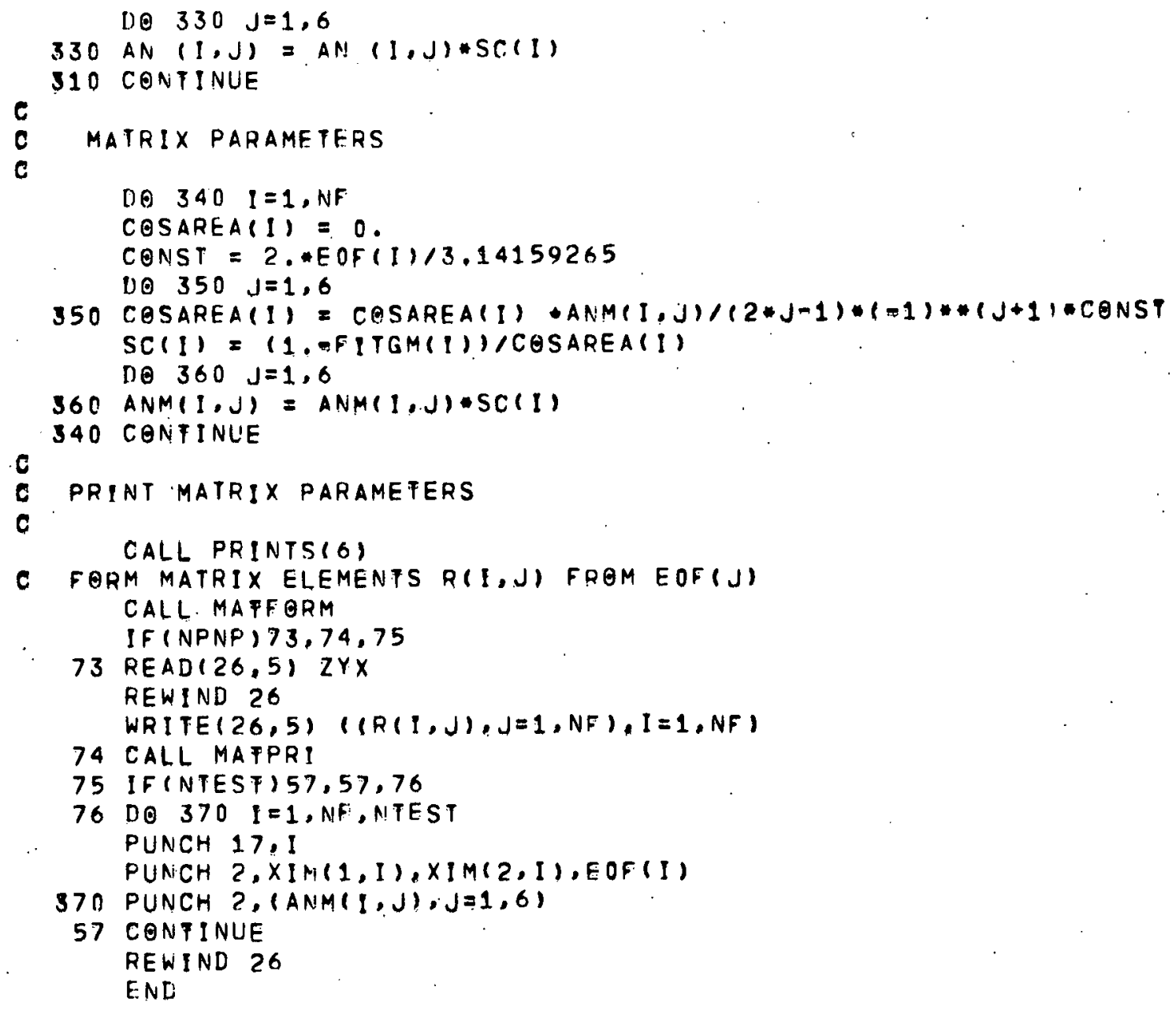

2. Printout of Subroutine MATFORM

SUBROUTINE MATFORM.

DIMENSION! R(128,128),EOF(128), ANM(12,,10),XIM(2,128),AN(30,10). $X X I(2,30), E O P(30), F I P G(30), \operatorname{COSAREA}(12 B), S C(128)$

c

DIMENSION RELG(30), ERG(128), TRANS $(128), B(8), E R G P(128)$

COMMON/12345/EOF,R, ANM, NF, XIM, AN, XI, EOP, AMEV,HALFA,LC,NFRI, NP, NC, XFITG, COSAREA, SC, NDEL, SUM, RELG, ERG,ERGP, TRANS, NTRAN, B, JA

c

DQ $31=1, \mathrm{NF}$

DO $3 J=1$, NF

$c$

$3 H(J, J)=0.0$

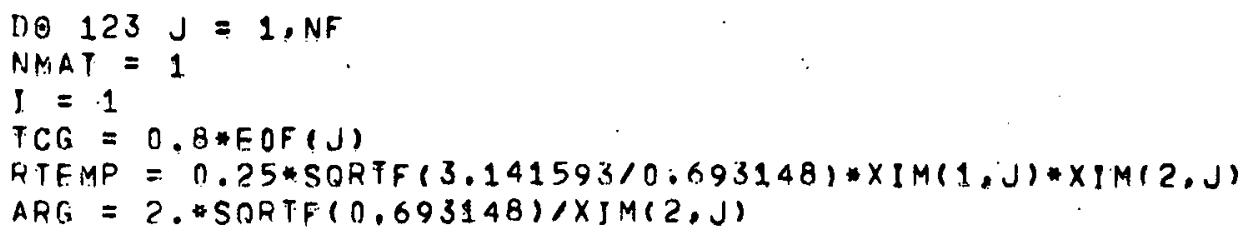

C 


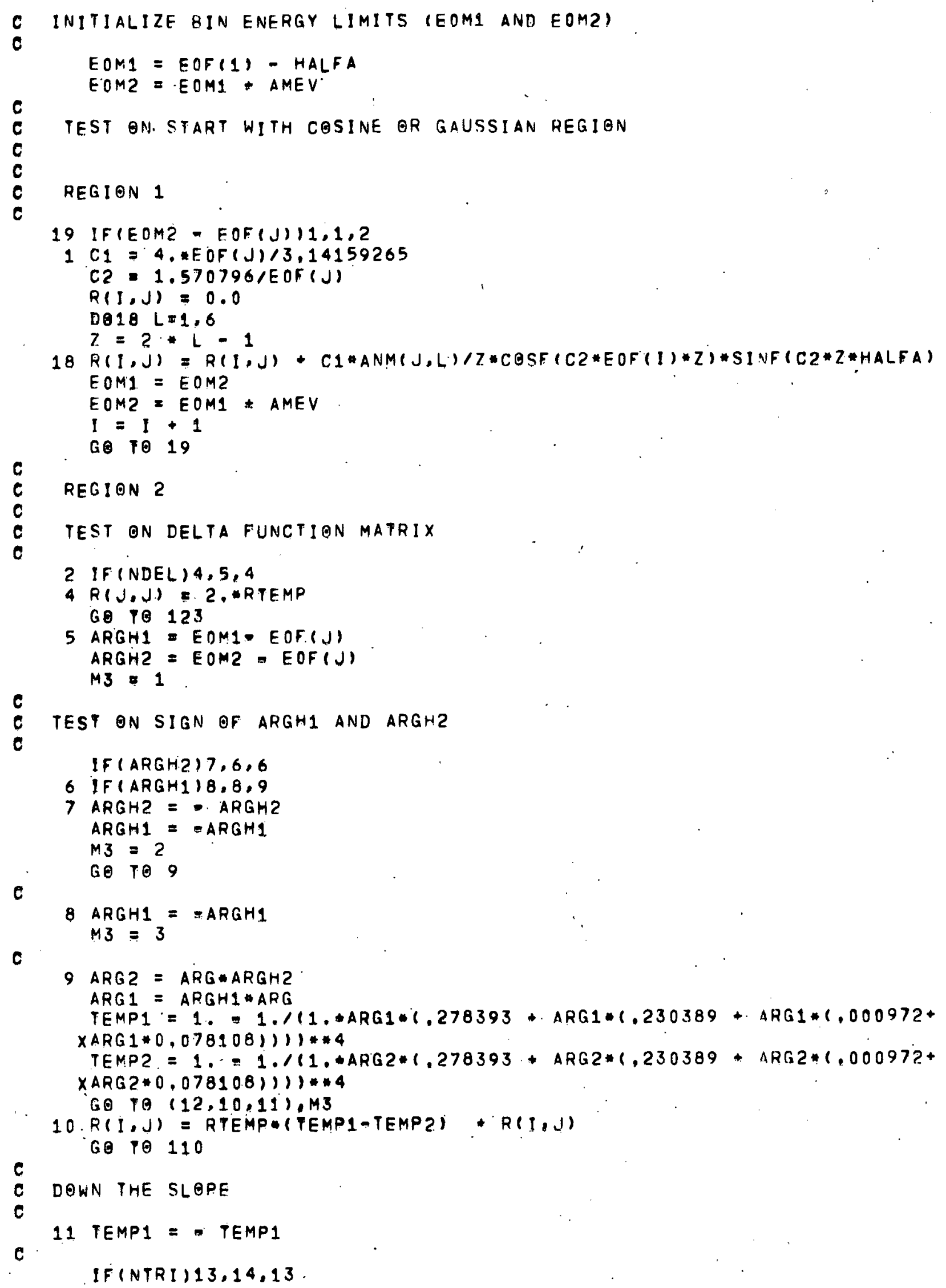


C

13 TEMP2 $=1$. GO TO 14

c

C

12 IF (NTRI) $15,14,15$

$14 R(J, J)=R T E M P *(T E M P 2-T E M P q) * R(1, J)$

IF $(R(I, J)-1, O E-4) 15,110,110$

110 EOM1 = EOM2

EOM2 = EOM2 $\triangle A M E V$ $I=1+1$

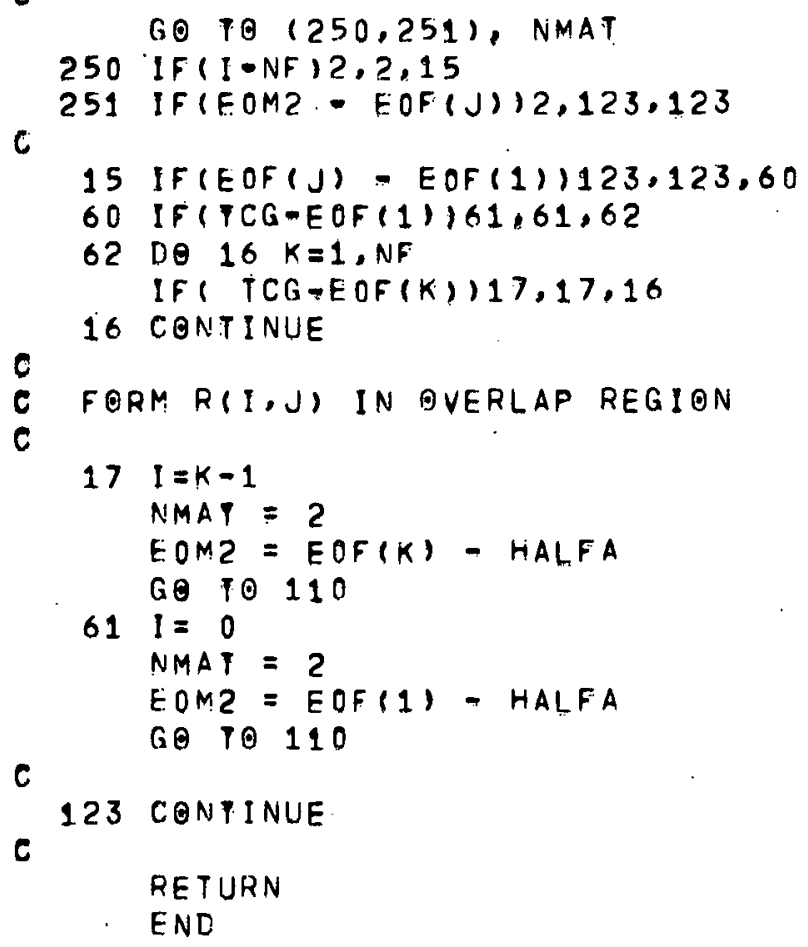

3. Printout of Subroutine PRINTS(NUM)

c

SURROUTINE PRINTS(NUM)

DIMENSION R(128,128), EOF (128), ANM(128,10),XIM(2,128),AN(30,10), . $X \times I(2,30), E O P(30), F I T G(30), C O S A R E A(128), S C(128)$

DIMENSION RELG(30), ERG(128), TRANS(128), B(8), ERGP(128)

C COMMON/12345/EOF,R, ANM, NF, XIM, AN,XI,EOP, AMEV,HALFA,LG,NTRI, NP, NC, XFITG, CESAREA, SC, NDEL, SUM, RELG,ERG, ERGP, TRANS, NTRAN, B, JA, NVMM

c

1 FORMAT(10HO EOP( $12,3 H)=F 10.5,5 \times 14 H T O T A L$ COIJNTS $=E 13.5,3$ $\times X 22$ HRELATIVE GAUSS. AREA $=F 10,5$,

2 FORMAT(1H1 3X1HI 8X6HERG(I) $7 \times$ 7HERGP(I) $4 \times 23 H(1 / R F A C T)$ (EP(G)/EP(G X) /(1H I4,2F14.5.E2S.5)

3 FORMAT(1H1 $26 \times 19$ HCOSINE CEEFFICIENFS /3X1HN 2X11HENFRGY,.MEV $7 X$ $\times 4 H A(1) 9 \times 4 H A(2) 9 \times 4 H A(3) 9 \times 4 H A(4) 9 \times 4 H A(5) 9 \times 4 H A(6)$, $X(1 H O[3, F 13,5,6 E 13,51)$

4 FORMAT 1 H $17 X$ 26HFITTED GAUSSIAN PARAMETERS $26 \times 11 H C O S I N E$ AREA. X6HO N $2 \times 15 H \times(3)=E O P$, MEV $10 \times 4 H \times(1) 10 \times 4 H \times(2) B \times 7 H F I T G(N) /$ $X(1 H 015, F 17,5, F 14,5, F 14,6, F 15,5, E 15,5))$ 


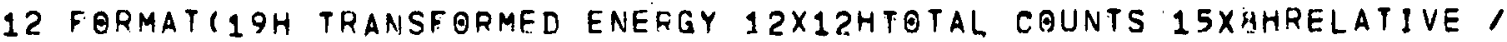
X6X1HN 5X6HEOP(N) 15X1OH(RAW.DATA) $12 \times 11$ HGAUSS. AREA 11

14 FQRMAT(1HOI6,F11,5,E25,5,F23,6)

16 FORMATI44HI UNFITTED NORMALIZED $\times 1 H 02 \times 1 H N 1 \times 1$ IHENERGY, MEV $9 \times 4 H A(1) 9 \times 4 H A(2)$ 9X4HA(3) $9 \times 4 H A(4)$ 9X $\times 4 H A(5)$ 9X4HA(6), (1HO $13, F 13,5,6 E 13.5))$

13 FORMATIIHI $10 X$ 58H NORMALIZED GAUSSIAN AREA OUARTIC COEFFICIENTS XFROM LSQPOL, 1 HO $10 \times 4 H B(1) 14 \times 4 H B(2) 14 \times 4 H B(3) 14 \times 4 H B(4)$. $X 4 H B(5) \quad 1 / 5 E 18.5,5(1), 10 \times$ 58H NORMALIZED COSINE AREA OUARTIC COEFF XICIENTS FROM LSOPOL /1HO $10 X$ 4HAPN) $14 X$ 4HB(1) $14 X 4 \mathrm{HB}(2) 14 \times 4 \mathrm{HB}$ $X(3) 14 X \quad 4 H B(4) 14 X 4 H B(5), 1$

5 FORMAT(1HO 1OX 2HAI (1, 1H) E20,5,4E18.5)

6 FORMAT (1.H14OX.MAFRIX COEFFICIENTS, SCALING FACTORS, AND PARAMETERS $X$ /1H02X1HN2X11HENERGY, MEV 9X4HA(1) $9 \times 4 H A(2)$ 9X4HA(3) $9 \times 4 H A(4) 9 X$ $\times 4 H A(5)$ 9X4HA(6) $6 \times 5 H S C(N)$ 9X4HX(1) $9 \times 4 H \times(2)$,

E $x$ $(1 H 013, F 13,5,6 F 13,8,3 F 13,6))$

Go $10(10,20,30,40,50,60), N U M$

10 IF (NP) $72,39,72$

3.9 IF (NVMM) 41, 42, 41

41 IF(NC-1) $43,43,44$

43 PRINT 12

44 PRINT $14, N C, E O P(N C), S U M, R E L G(N C)$ RETURN

42 PRINT 1,NC,EOP(NC), SUM,RELG (NC) RETURN

20 IF (NTRAN)72,51,72

51 PRINT 2, (1,ERG (I),ERGP(I), TRANS $(1), I=1, L C)$ RETURN

30 IF (NP) 72,58,72

58 PRINT $3,(I, E O P(I),(A N(I, J), J=1,6), 1 \geq 1, N C)$ RETURN

40 IF (NP) 72,65,72

65 PRINT $4,(1, E O P(I), \times I(1, I), \times I(2, I), F I T G(I), C O S A R E A(I), I=1, N C)$ PRINT $16,(I, E O P(1),(A N(I, J), J=1,6), I=1, N C)$

PRINT.13, (B(I), I =1,5) RETUR.N

50 IF (NP) 72,8B,72

88 PRINT $5, J A,(R(I), I=1,5)$ RETURN

60 IF (NP) 72,71,72

71 PRINT6, (I, EOF (I), (ANM(I,J),J=1,6),SC(I),XIM(1,I),XIM(2,I),I =1,NF)

72 RETURN END

4. Printout of Subroutine MATPRI

SUBROUTINE MATPRI

DIMENSI BN R(128,128), EOF (128), ANM(128,10), XIM(2,128),AN(30,10),

$X X I(2.30), E O P(30), F I T G(30)$, COSAHEA $(128), S C(128)$

UIMENSION RELG(30), ERG(128), TRANS(128), B(8), ERGP(128)

COMMON/12345/EOF, R, ANM, NF, XIM, AN, XI, FOP, AMEV,HALFA,LC,NTRI, NP, NC, XFITG, COSAREA, SC, NDEL, SUM, RELG, ERG,ERGP, TRANS, NTRAN, B, JA

PRINT 1.6

16 FORMATIIHI $50 X$ RESPONSE MATRIX * MID ENERGY (MEV)* //)

$N 1=1$

$N 2=8$

IF (N2-NF) 2,2,1

$1 N 2=N F$ 


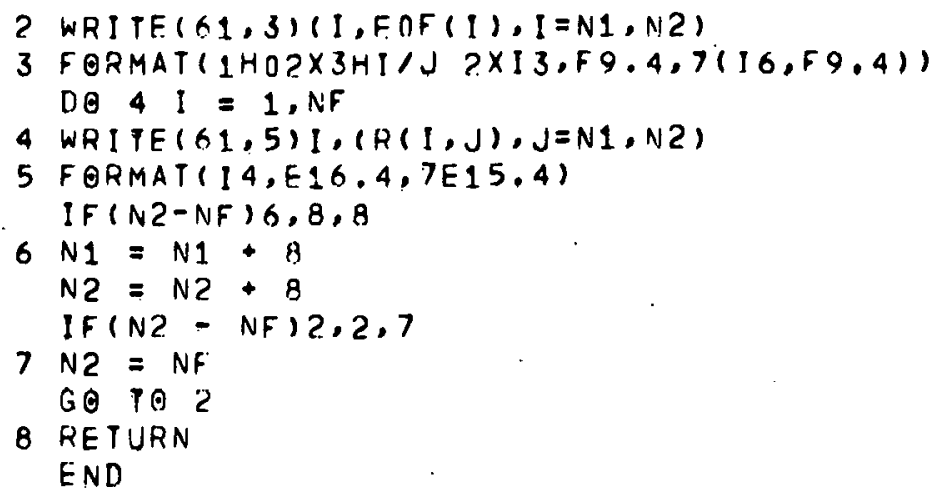

5. Printout of Subroutine VMM*

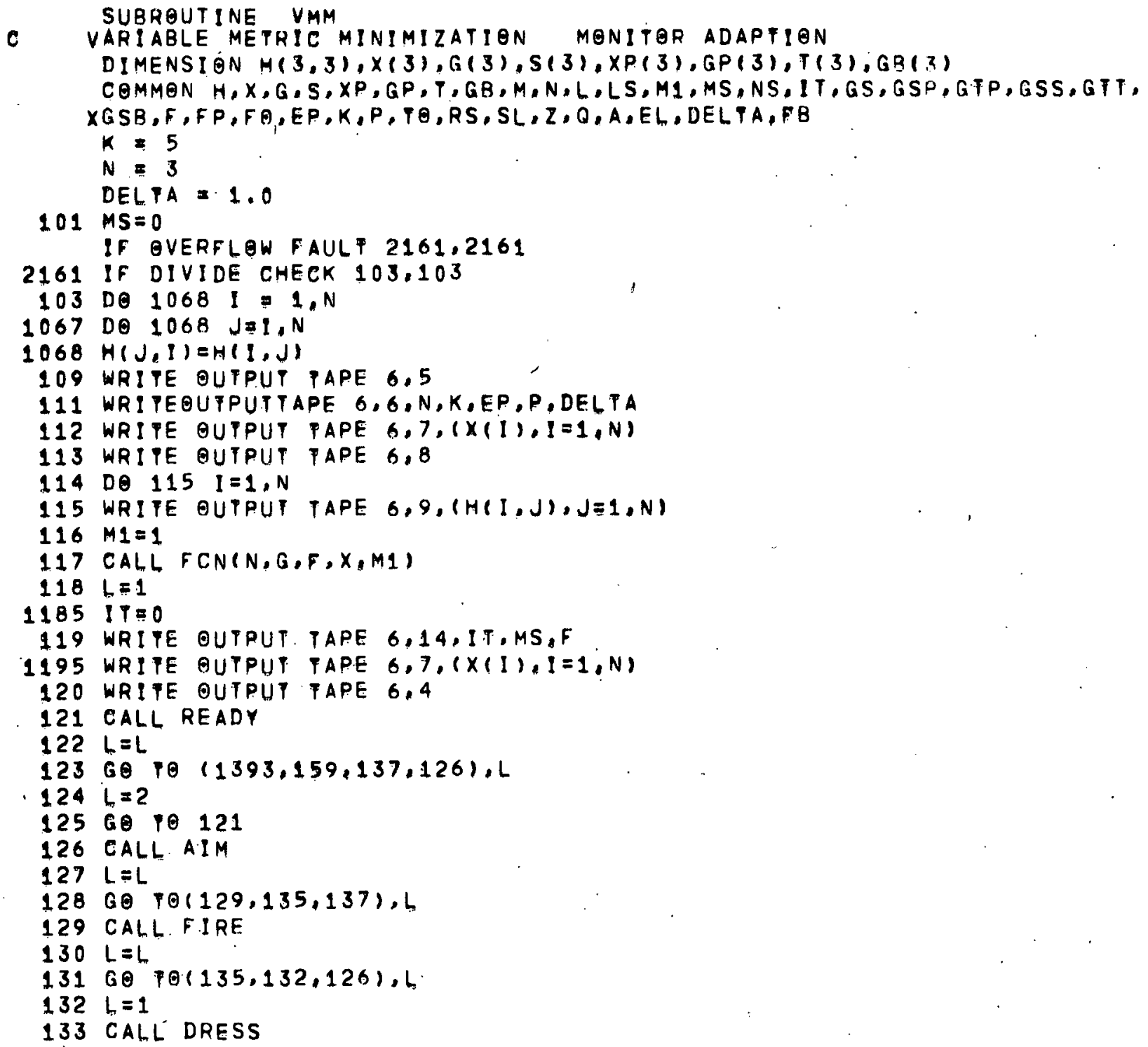

\footnotetext{
*Special "equip" cards are needed to run this IBM 704 subroutine on CDC 3600.
} 


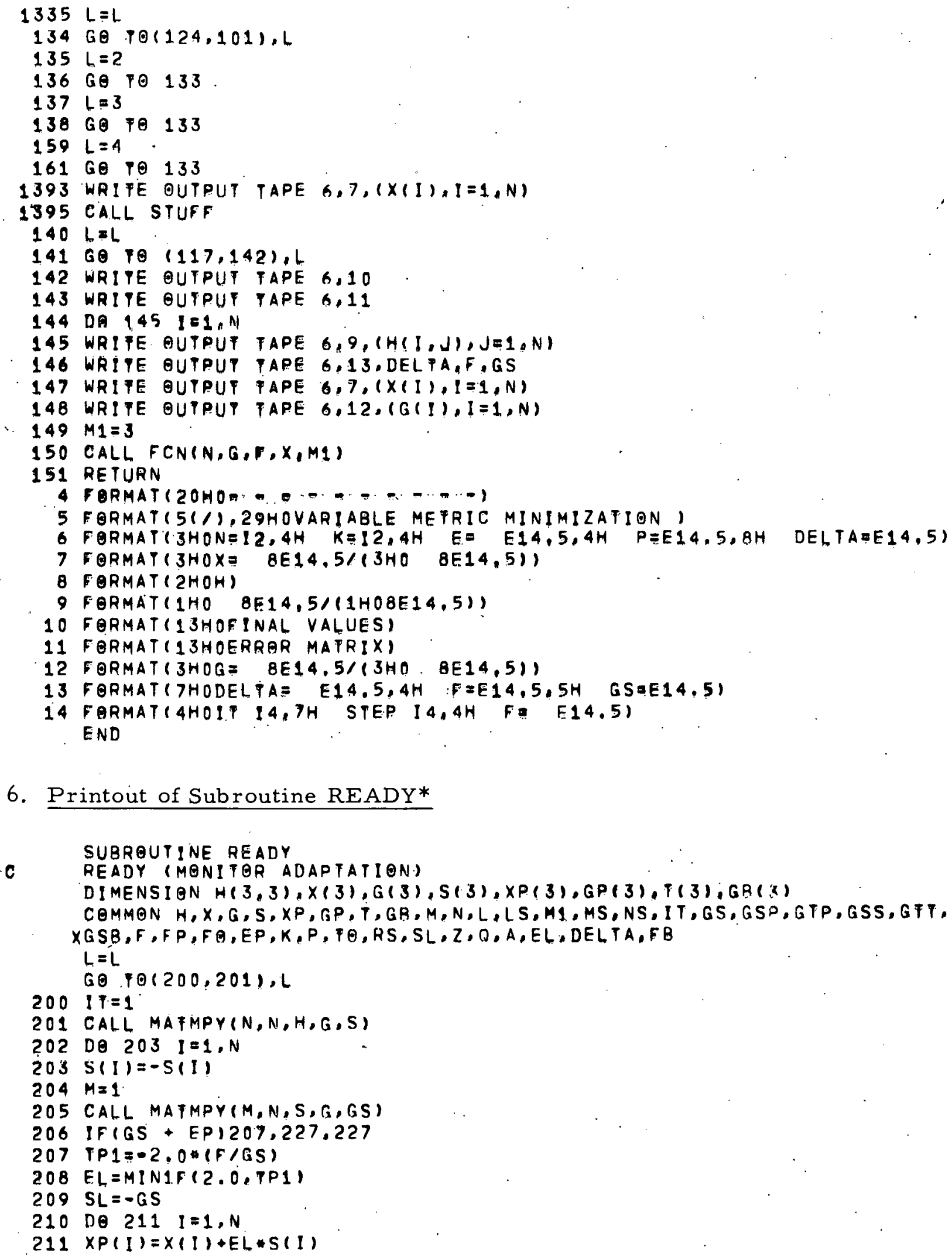

* Ibid. page 86 


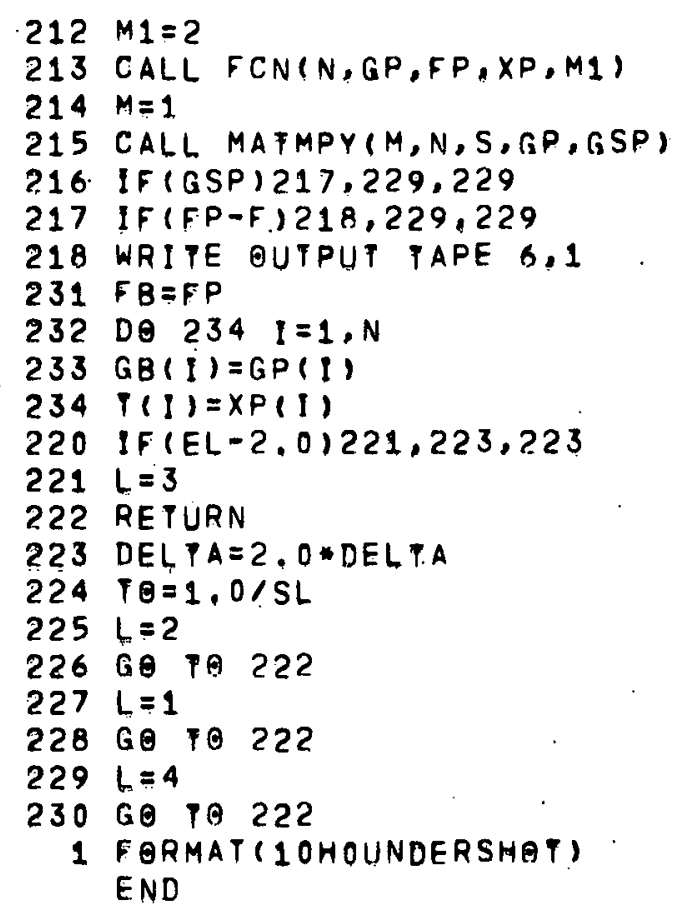

7. Printout of Subroutine AIM*

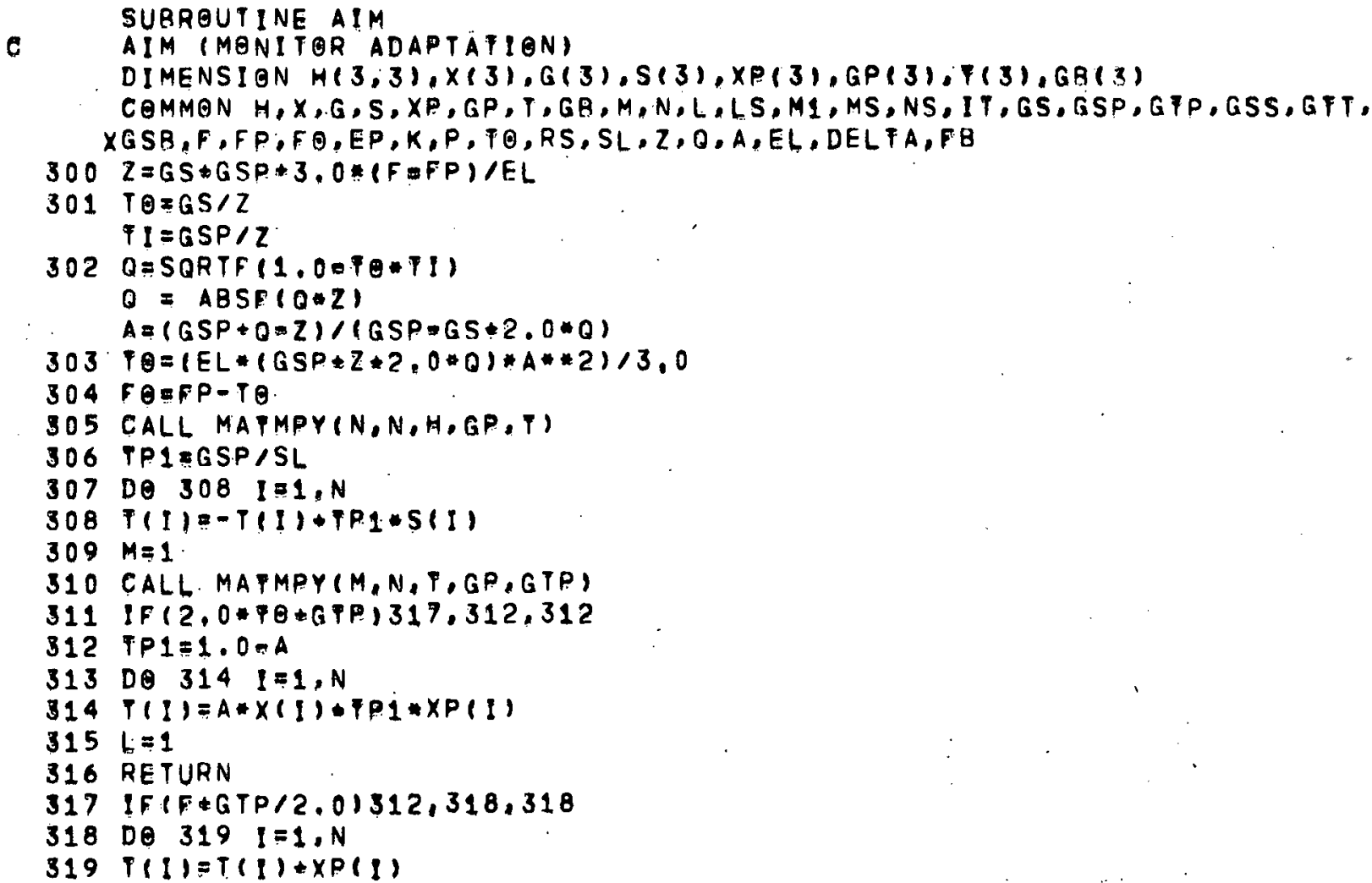

*Ibid. page 86 . 


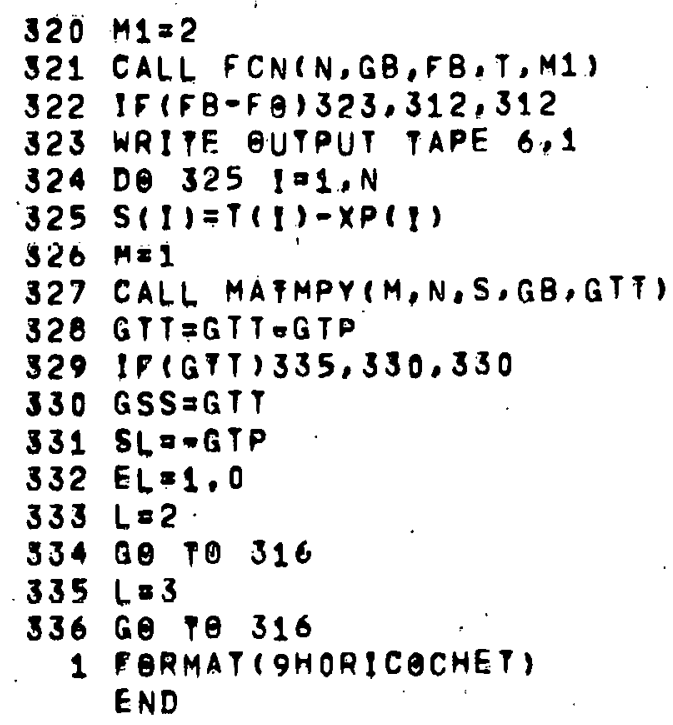

8. Printout of Subroutine FIRE*

c

SUBRAUTINE FIRE

C TIRE (MONITOR ADAPTAFION)

DIMENSION H(3, 3),X(3),G(3),S(3),XP(3),GP(3), $(3), G B(3)$

COMMON $H, X, G, S, X P, G P, T, G B, M, N, L, L S, M I, M S, N S, I T, G S, G S P, G F P, G S S, G T T$, $X G S B, F, F P, F \theta, E P, K, P, F \theta, R S, S L, Z, O, A, E L, D E L T A, F B$

$400 \quad M 1=2$

401 CALL FCN(N,GB,FB,T,M1)

$402 M=1$

403 CALL. MATMPYPM,N,S,GB,GSB\}

404 TPI MMINIF (F,FP)

405 IF (PPI-FB+EP) 418,406,406

$406 P P 1=A /(1,0=A)$

$407 P P 2=(1.0=A) / A$

$408 Y Q=Q S B *(T P 1=P P 2)$

409 IF (ABSF $(T \theta)=01413,410,410$

110 GSS $=2.0 * 0$

$111 L \equiv 1$

412 RETURN

413 GSSE.TO+2,0.0

414 DO 415 I:1,N

415 Q(I)=(GB(I)-Q(1) $+P P 1+(G P(1)-G B(1))+7 P 2$

416. $L a 2$

41760 PO 412

418 IF (F)FP $1419,428,428$

119 WRIPE OUTPUT PAPE 6.1

120 EL $11.0 . A)$ EL

$421 T P=F B$

422 CSPEGSB

423 DO $425 \quad I \equiv 1, N$

$.424 \times P(1)=T(1)$

$425 G P(I)=G B(1)$

$426 \quad h=3$

42760.10412

* Tbid. page 86. 


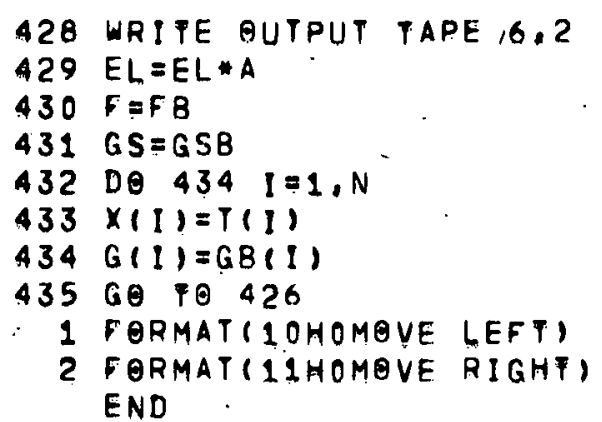

9. Printout of Subroutine DRESS*

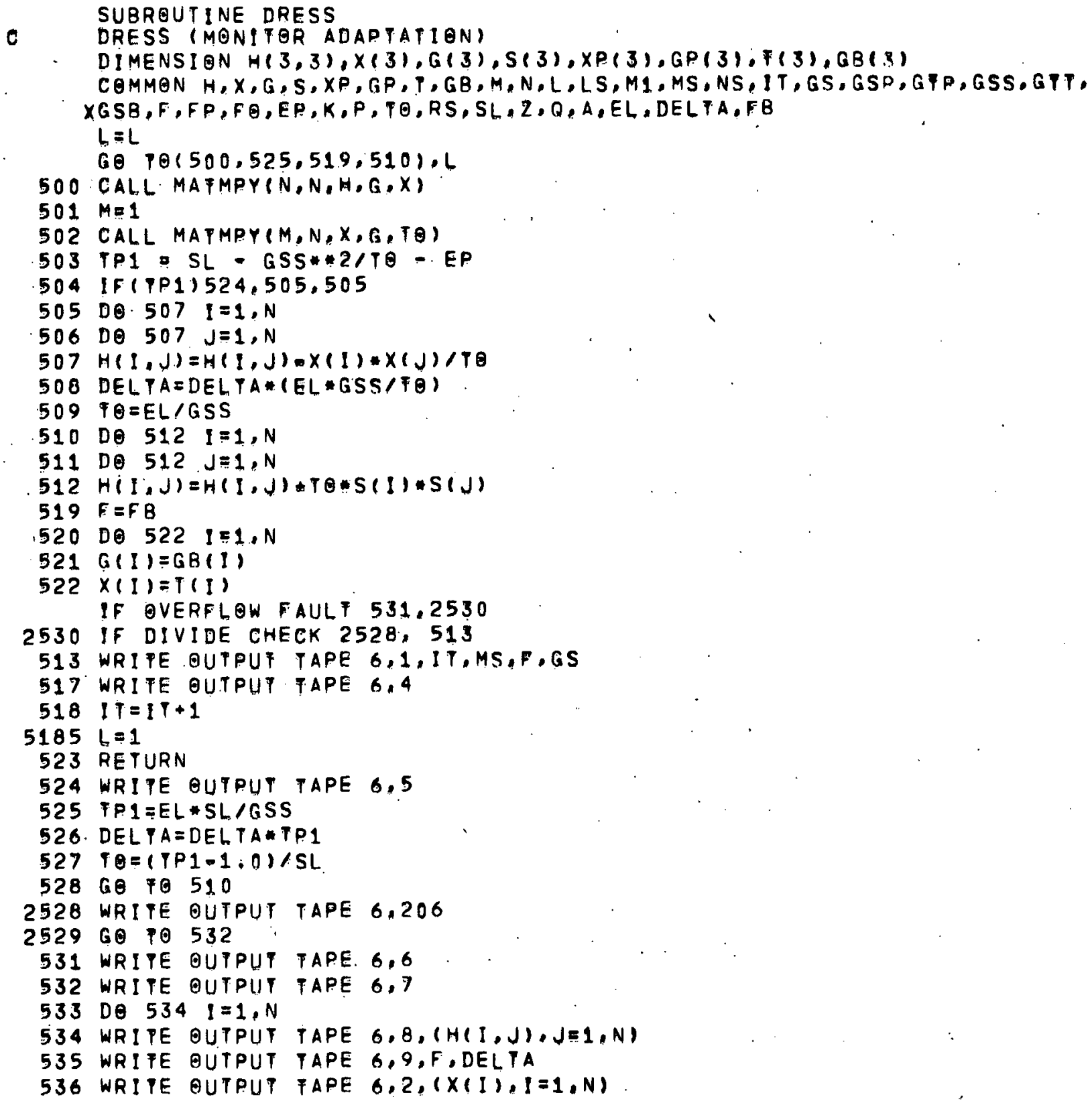

* Ibid. page 86 . 


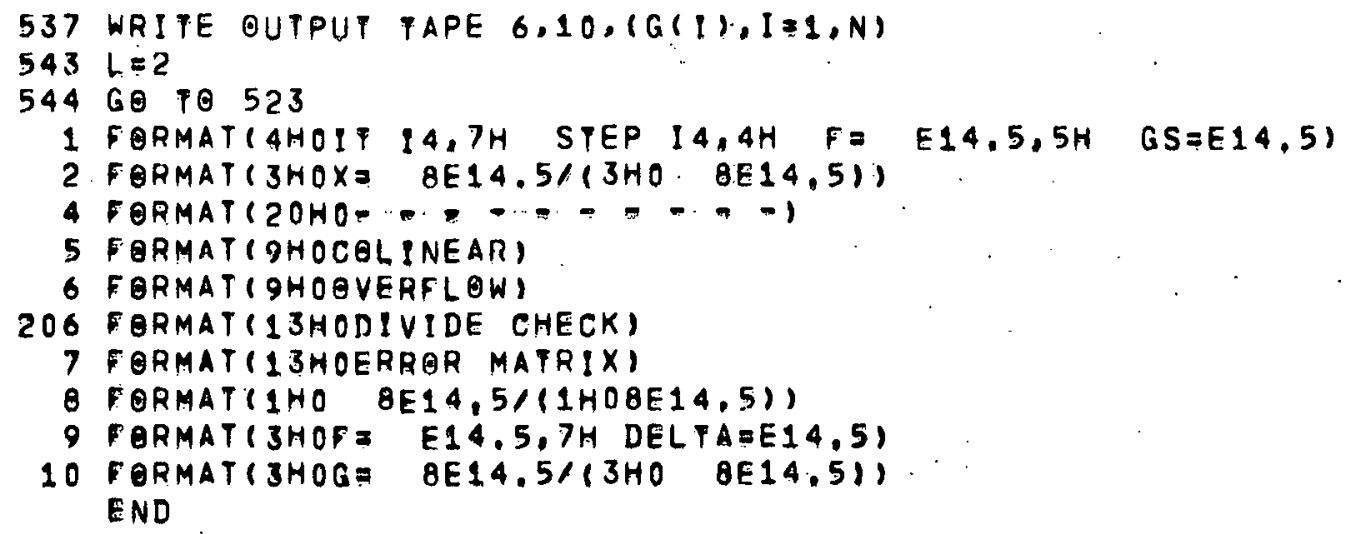

10. Printout of Subroutine STUFF*

SUBRQUTINE SFUFF
C STUFF (MONITER. ADAPTATION)

DIMENSION H(3,3),X(3),G(3),S(3),XP(3),GP(3),P(3),GB(3)

COMMON H,X,G,S,XP,GP, T,GB,M,N,L,LS,MI,MS,NS,IT,GS,GSP,GTP,GSS,GTY,

$X G S B, F, F P, F O, E P, K, P, T O, R S, S L, Z, Q, A, E L, D E L P A, F B$

$600 \quad K=K-1$

$601 \quad F(k) 617,602,602$

$602 M S=M S+1$

$P=\operatorname{SORFF}(2, * F)$

620 WRITE OUTPUT TAPE 6,1,MS, DELTA,GS

$2603 T(1) \equiv R A N F \quad(=1)-0,5$

603 DO $604 \quad 1=2, N$

604 F I I $=$ RANF $(-1)-0,5$

605 CALL MATMPY $(N, N, H, T, S)$

$606 \quad M=1$

607 CALL MATMPY (M,N,S,T,FP1)

608 PPI $=S O R F F(T P 1)$

$609 E L=P / T P 1$

$610 D \theta 6111=1, N$

$611 \times(1)=X(1)+E L \# S(I)$

$612 M 1=2$

$614 L=1$

616 RETURN

$617 \mathrm{~L}=2$

$618 M S=0$

61960.70616

1 FORMATI13HORANDOM SPEP 84, AH DELTA=1PE14.5,5H GS=E1.4.5) END

11. Printout of Subroutine MATMPY $(M, N, H, G, S)^{*}$

SUBRQUTINE MATMPY $(M, N, H, G, S)$

C MATRIX MULTIPLICATION (MONIFOR ADAPYATION)

DIMENSION H(3, 3), G(3),S(3)

702 DO $703 \quad I=1, M$

$700 \mathrm{~S}(1)=0.0$

DO $703 \mathrm{~J}=1, \mathrm{~N}$

$703 S(I)=H(J, I) * G(J) * S(I)$

704 RËTURN

END

*Ibid. page 86. 
12. Printout of Subroutine $\operatorname{FCN}(M, G, F, B, M l)^{*}$

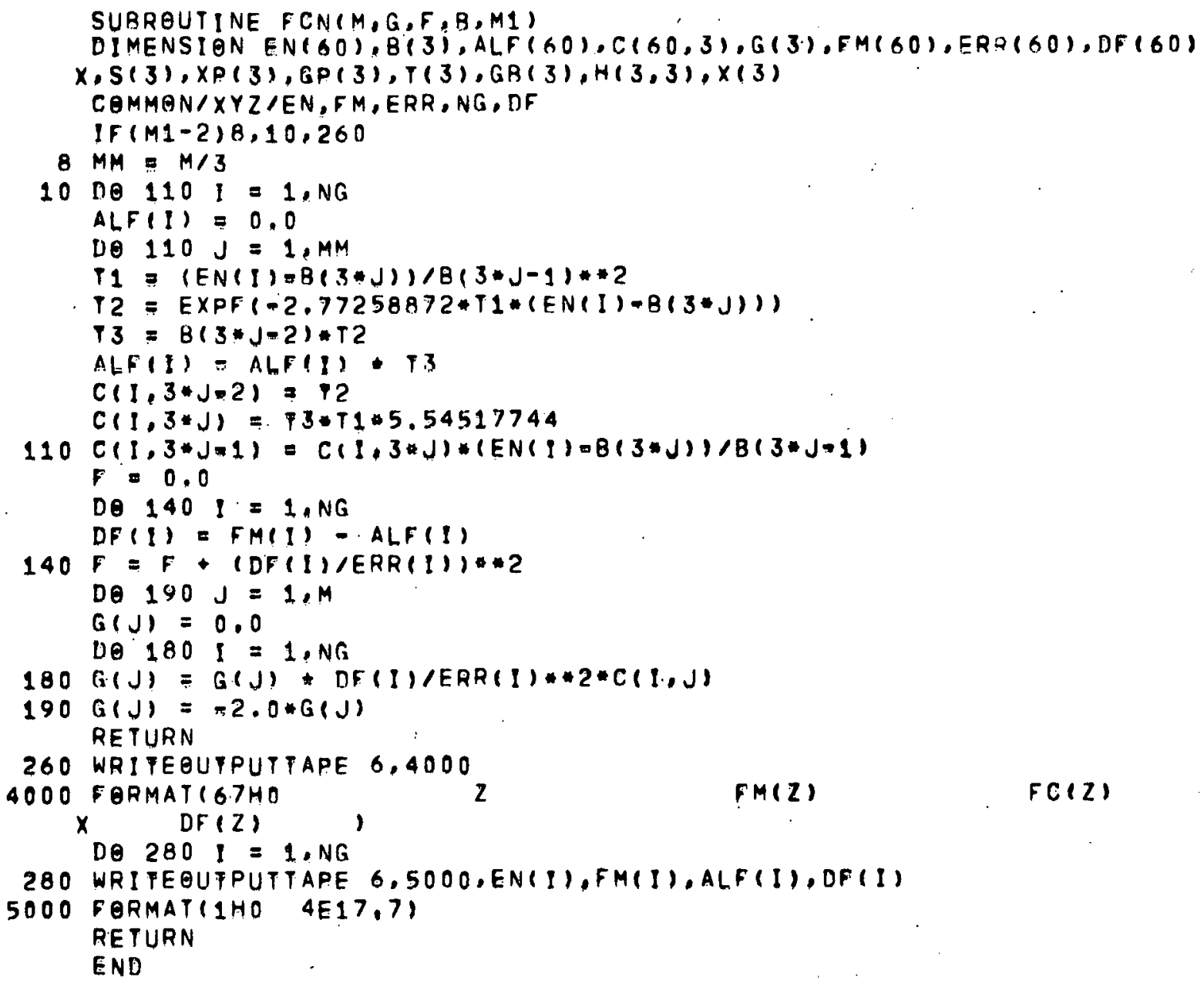

TAB LE B.III. WERM Sample Input**

\begin{tabular}{|c|c|c|c|c|c|c|c|c|c|c|c|}
\hline \multirow[t]{2}{*}{$\begin{array}{l}0.0 \\
.060 \\
.300 \\
.900 \\
.0014 \\
.0619 \\
.376 \\
2.10\end{array}$} & $\begin{array}{l}.005 \\
.065 \\
.350 \\
.950 \\
.0064 \\
.0667 \\
.470 \\
2.30\end{array}$ & $\begin{array}{l}.010 \\
.070 \\
.400 \\
1.0 \\
.0114 \\
.0715 \\
.574 \\
2.51\end{array}$ & $\begin{array}{l}.015 \\
.075 \\
.450 \\
1.5 \\
.0163 \\
.0763 \\
.087 \\
5.02\end{array}$ & $\begin{array}{l}.020 \\
.080 \\
.500 \\
2.00 \\
.0213 \\
.0811 \\
.809 \\
8.24\end{array}$ & $\begin{array}{l}.025 \\
.085 \\
.550 \\
2.5 \\
.0263 \\
.0859 \\
.940 \\
12.2\end{array}$ & $\begin{array}{l}.030 \\
.090 \\
.600 \\
3.0 \\
.0313 \\
.0908 \\
1.08 \\
16.7\end{array}$ & $\begin{array}{l}.035 \\
.095 \\
.050 \\
3,5 \\
.0363 \\
.0957 \\
1,23 \\
22.0\end{array}$ & $\begin{array}{l}.040 \\
.100 \\
.700 \\
4.0 \\
.0424 \\
.101 \\
1.38 \\
27.9\end{array}$ & $\begin{array}{l}.045 \\
.150 \\
.750 \\
4.5 \\
.0474 \\
1154 \\
1.55 \\
34.5\end{array}$ & $\begin{array}{l}.050 \\
.200 \\
.800 \\
5.0 \\
.0523 \\
.218 \\
1.73 \\
41.6\end{array}$ & $\begin{array}{l}.055 \\
.250 \\
.850 \\
.0571 \\
.292 \\
1.91\end{array}$ \\
\hline & .0179 & .1356 & $698 \div 21$ & 0 & $\begin{array}{l}188332 \\
1\end{array}$ & 31 & 128 & 0 & $1=1$ & 1. & 0 \\
\hline $\begin{array}{l}9 / 67 \\
0,148\end{array}$ & $\begin{array}{r}16-1 \\
216 * 2\end{array}$ & $\begin{array}{r}87 \\
110.5\end{array}$ & $S^{S 1} .12$ & & 7 & & & & & & \\
\hline 10 & & 1014 & 1028 & 87 & $\begin{array}{l}042 \\
126\end{array}$ & 1056 & 10.70 & & 084 & 1098 & 111 \\
\hline & & 1154 & 1168 & & 182 & 1196 & 1210 & & 224 & 1238 & 125 \\
\hline
\end{tabular}

*Ibid. page 86.

$* *$ Overflow ( $>72$ columns) of input data printed on following line. 
TABLE B.III. (Contd.)

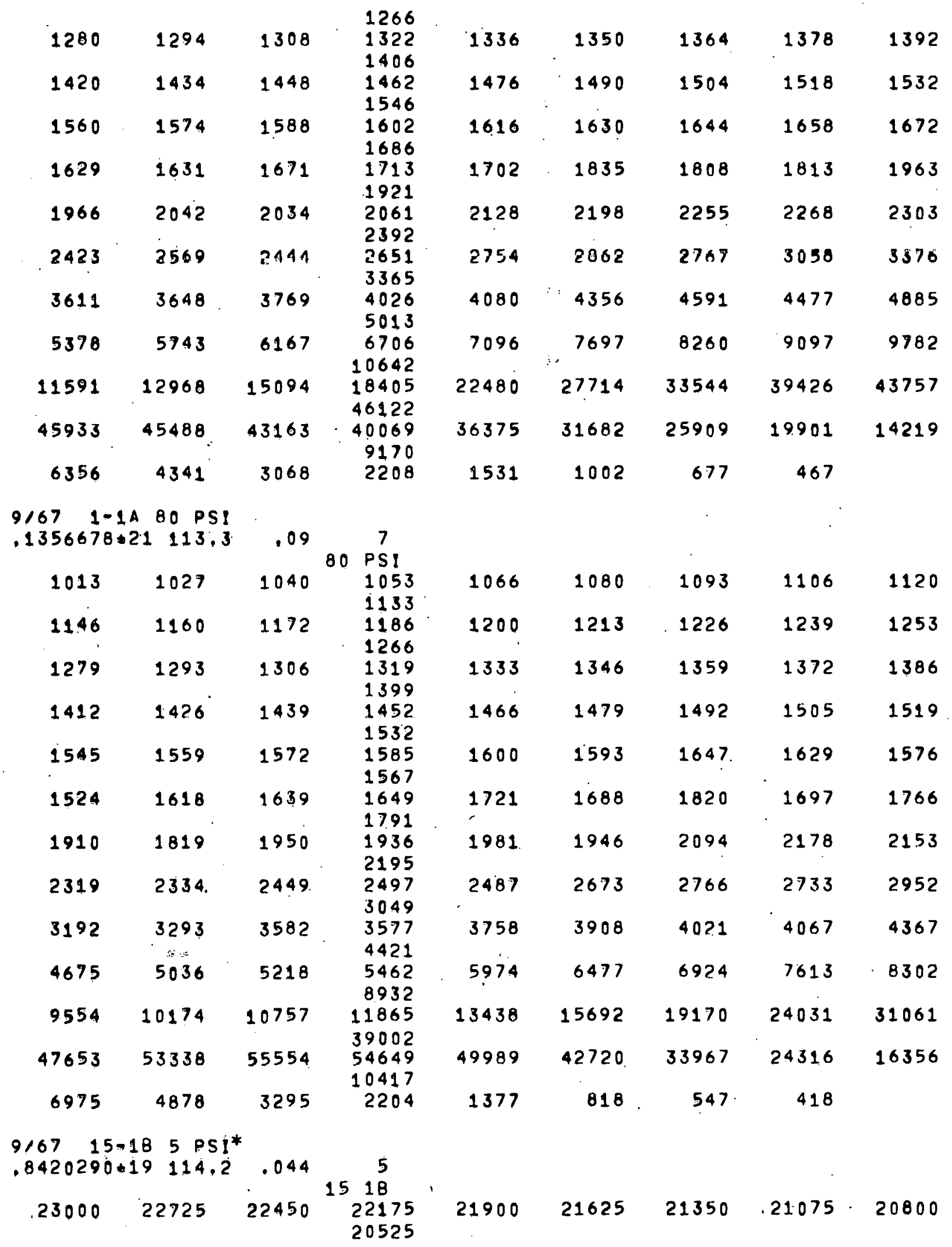

* The above is only a sample of the total response function input data required. 


\begin{tabular}{|c|c|c|c|c|c|c|c|c|}
\hline 20250 & 19975 & 19700 & 19425 & 19150 & 18875 & 18600 & 18325 & 17793 \\
\hline 17145 & 16885 & 16728 & $\begin{array}{l}16333 \\
14533\end{array}$ & 15987 & 15831 & 15440 & 15052 & 14814 \\
\hline 14439 & 14236 & 13478 & $\begin{array}{l}13734 \\
12005\end{array}$ & 13351 & 13035 & 12845 & 12591 & 12196 \\
\hline 11703 & 11531 & 11067 & $\begin{array}{r}10940 \\
9178\end{array}$ & 10604 & 10156 & 9949 & 9661 & 9295 \\
\hline 9037 & 8759 & 8665 & $\begin{array}{l}8387 \\
7637\end{array}$ & 8357 & 8100 & 7833 & 7588 & 7674 \\
\hline 7448 & 7205 & 7036 & $\begin{array}{l}6776 \\
5851\end{array}$ & 6818 & 6581 & 6365 & 6252 & 6087 \\
\hline 5594 & 5450 & 5451 & $\begin{array}{l}5264 \\
3900\end{array}$ & 5019 & 4816 & 4581 & 4380 & 4223 \\
\hline 3970 & 3591 & 3284 & 2445 & 3031 & 2785 & 2718 & $\cos 1$ & 2300 \\
\hline 2360 & 2220 & 2197 & $\begin{array}{l}2230 \\
1967\end{array}$ & 2067 & 2025 & 1981 & 1973 & 1880 \\
\hline 1796 & 1777 & 175 & $\begin{array}{l}1093 \\
2608\end{array}$ & 1852 & 17.95 & 1012 & 1459 & 2192 \\
\hline 3240 & 4477 & 6502 & $\begin{array}{r}8458 \\
62\end{array}$ & 8066 & 5401 & 2176 & 569 & 145 \\
\hline 34. & 44 & 31 & 32 & 31 & 30 & 10 & 34 & \\
\hline
\end{tabular}


APPENDIX C

ITERATE Code Listing

TABLE C.I. ITERATE Input

\begin{tabular}{|c|c|c|c|}
\hline Card & Variable & Format & Description \\
\hline 1 & NTYPE $=2$ & 16 & $\begin{array}{l}\text { Generate SME matrix via MAT } 2 \text { from } \\
\text { magnetic tape. }{ }^{2}\end{array}$ \\
\hline \multirow[t]{2}{*}{2} & $N \leq 120$ & I6 & Matrix size, $\mathrm{N} \times \mathrm{N}$. \\
\hline & NPRINT & I6 & $\begin{aligned} l= & \text { do not print } S M E \text { matrix or sym- } \\
& \text { metric } S M E \text { matrix. } \\
2= & \text { do print } S M E \text { matrix or symmet }- \\
& \text { ric SME matrix. }\end{aligned}$ \\
\hline \multirow[t]{10}{*}{$\cdot$} & NGEN & I6 & $\begin{array}{l}1=\text { SME matrix input by rows. } \\
2=\text { first row and first column input. }\end{array}$ \\
\hline & MSYM & I6 & $\begin{array}{l}1=\text { do not symmetrize SME matrix. } \\
2=\text { symmetrize. }\end{array}$ \\
\hline & ITMAX & I6 & Output control parameter. Results \\
\hline & NOUT & I6 & $\begin{array}{l}\text { printed IT modulo NOUT, where IT is } \\
\text { the iteration number. }\end{array}$ \\
\hline & NERR & I6 & $\begin{aligned} I= & \text { input relative errors of } W(I) \\
& \text { Under this option, } \operatorname{ER}(\mathrm{I}) \text { is read } \\
& \text { in after } \mathrm{Xl}(\mathrm{I}) .\end{aligned}$ \\
\hline & $\cdot$ & . & $\begin{aligned} 2= & \text { same relative errors for all } \\
& W(I)\end{aligned}$ \\
\hline & . & & $\begin{aligned} 3= & \text { error formed in program as } \\
& 1 / \sqrt{W(I)} .\end{aligned}$ \\
\hline & NU & I6 & $\begin{array}{l}\text { Number of iteration sets to be } \\
\text { punched. }\end{array}$ \\
\hline & INCH & I6 & Initial channel for energy formation. \\
\hline & A & $F 12.0$ & $\mathrm{MeV} /$ channel (for energy printout). \\
\hline 3 & $\begin{array}{l}{[\mathrm{Nl}(\mathrm{I}), \mathrm{I}=1, \mathrm{NU}]} \\
\mathrm{NU} \leq 9\end{array}$ & 16 & $\begin{array}{l}\text { Which iteration numbers to punch for } \\
\text { input to PSNS. }\end{array}$ \\
\hline 4,5 & $W(I), I=1, N$ & $6 \mathrm{~F} 12.0$ & $\begin{array}{l}\text { Components of output vector of } \\
\text { detection system. }\end{array}$ \\
\hline$\cdots$ & $\mathrm{Xl}(\mathrm{I}), \mathrm{I}=1, \mathrm{~N}$ & $6 \mathrm{~F} 12.0$ & $\begin{array}{l}\text { Components of initial vector used to } \\
\text { initiate iteration procedure. }\end{array}$ \\
\hline
\end{tabular}

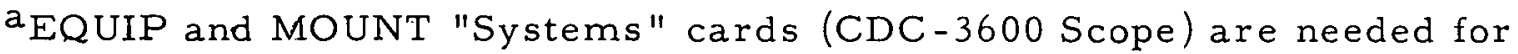
magnetic-tape input of SME matrix via MAT2. 
1. Printout of Program ITERATE

PROGRAM ITERATE

DIMENSION R(120), SUMN(120), XX(128)

COMMON /1/ SME(120,120)/2/SS(120,120),WNU(120),W(120),ER(120),

$X X(120), N, N P R I N T, M S Y M, I T M A X, N A U T, X 1(120), N 1(10), N U, I N C H$

C BANK, $(0), 11 /, 1$ I TERATE), /2/

c

1 READ 2, NTYPE

2 FORMAT (1216)

$I X=1$

IF (EOF, 60)200,201

200 CALL EXIT

2ก1. CANPINUE

GO TO $(3,4) N T Y P E$

3 CALL MAF1

GO TO 5

4 CALL MAF?

5 CONFINUE

$N N X=N+I N C H=1$

$N X=I N C H=1$

$00300 I=1, \mathrm{NX}$

$300 \times \times(1)=0$.

- GO TO 7,6$) \mathrm{MSYM}$

6 CALL MAFSYM

GO TO 10

c

S MATRIX IS SYMMETRICAL

c

7 DO $B \quad I=1, N$

$8 W N U(1)=W(1)$

DO. $9 I=1, N$

DO $9 J=1, N$

$9 \operatorname{SS}(1, J)=\operatorname{SME}(1 ; J)$

c

C

FORM BOUND FOR ARRESTING CRITERION

$10 B N D=0$

DO $100 \quad I=1, N$

100 BNDaBND*(ER(I)*WNU(1))* *2

GO FO(13,11)NPRINT

11 PRINT 12 .

12 FORMAT(1H117XI7HORIGINAL S MATRIX//)

CALL MPRINT (SME N)

13 GO TO(17,14IMSYM

14 GO PO(17,15) NPRINT

15 PRINT 16.

16 FERMAT(1H117X19HSYMMETRIC SS MATRIX//)

CALL MPRINT $(S S, N)$

C

17 CONTINUE

BEGIN ITERATI ON PROCEEDURE

PRINT 18,

18 FORMAT $(1 H 1)$

$I T=1$

$N C Q N V=2$ 
C

I9 CONTINUE

C

C

2? SUM1 $=0$.

i) $024 \quad I=1, N$.

SUM $=0$.

Do $23 \quad J=1, N$

23. SUM $=S U M+S S(I, J) * X 1(J)$

$X(I)=X I(I) * W N U(I) / S U M$

$W(I)=S \cup M$

$H(I)=W N U(I)-W(1)$

SUM1 $=$ SUM1+R(1)*2

24 SUMN(I) $=$ SUMI

IF (SUMN(N) - SND) 25,25,27

25 PRINT 26, IT, BND, SUIMN(N)

26 FORMATIIHO//9X5OHARRESTING CRITERION SATISFIED ON ITFRATIQN NUMBER

1 I $6.1 / 9 \times 6$ HHOUND $=F 11.4,3 \times 5 H N O R M=E 11.4$,

2.1 $1 \times 5 H W P R I M E 12 \times 1$ HR $13 \times 4 H N O R M / / 1$

$N X=I N C H$

$00301 \cdot I=1, N$

$X X(N X)=X 1(1)$

$301 N X=N X+1$

PUNCH $280,(X X(1), I=1, N N X)$

REWIND 26

NCONV $=1$

Go TO 30

27 IF IX - NU) $278,278,277$

278 IF(IT - NIIX) I 277,279,277

$279 \mathrm{NX}=I \mathrm{NCH}$

D) $302 \quad I=1, N$

$X X(N X)=\times 1(1)$

$302 N X=N X+1$.

PUNCH $280,(X X(I), I=1, N N X)$

280 FORMAT (6F 12,0$)$

$I X=I X+1$

c

277 IF(XMODF(IT, NOUT) $34,28,34$

28 PRINT 29, IT

29 FQRMAT (1HO//9X17HITERATION NUMBER I6,//26X9HI9X1HX11X6HWPRIME12X1H $1 R 13 \times 4 H N O R M / /)$

C

$30 \quad D 031 \quad I=1, N$

31 PRINT $32, I, \times 1(1), W(I), R(1), \operatorname{SUMN}(1)$

32 FORMAT(I2], 4E 15.4)

C

$3360 T 0(1,34) N C O N V$

$34 I T=I T+1$

IF $(I T-I T M A X) 35,35,37$

35 DO $36 \quad 1=1, N$

$36 \times 1(1)=\times(I)$

lio TO 19

37 PRINT 38; ITMAX,BND, SUMN(N)

38 FORMAT 1 HO//9X4OHARRESTING CRITERION. NOT SATISFIED AFTER, I6,2X11HI

1TERATIONS. $/ 19 \times 6$ TROUND $=E 11.4,3 \times 5 H N O R M=E 11.4$ I

REWIND 26

GO $F \circ 1$

END 
2. Printout of Subroutine MATSYM

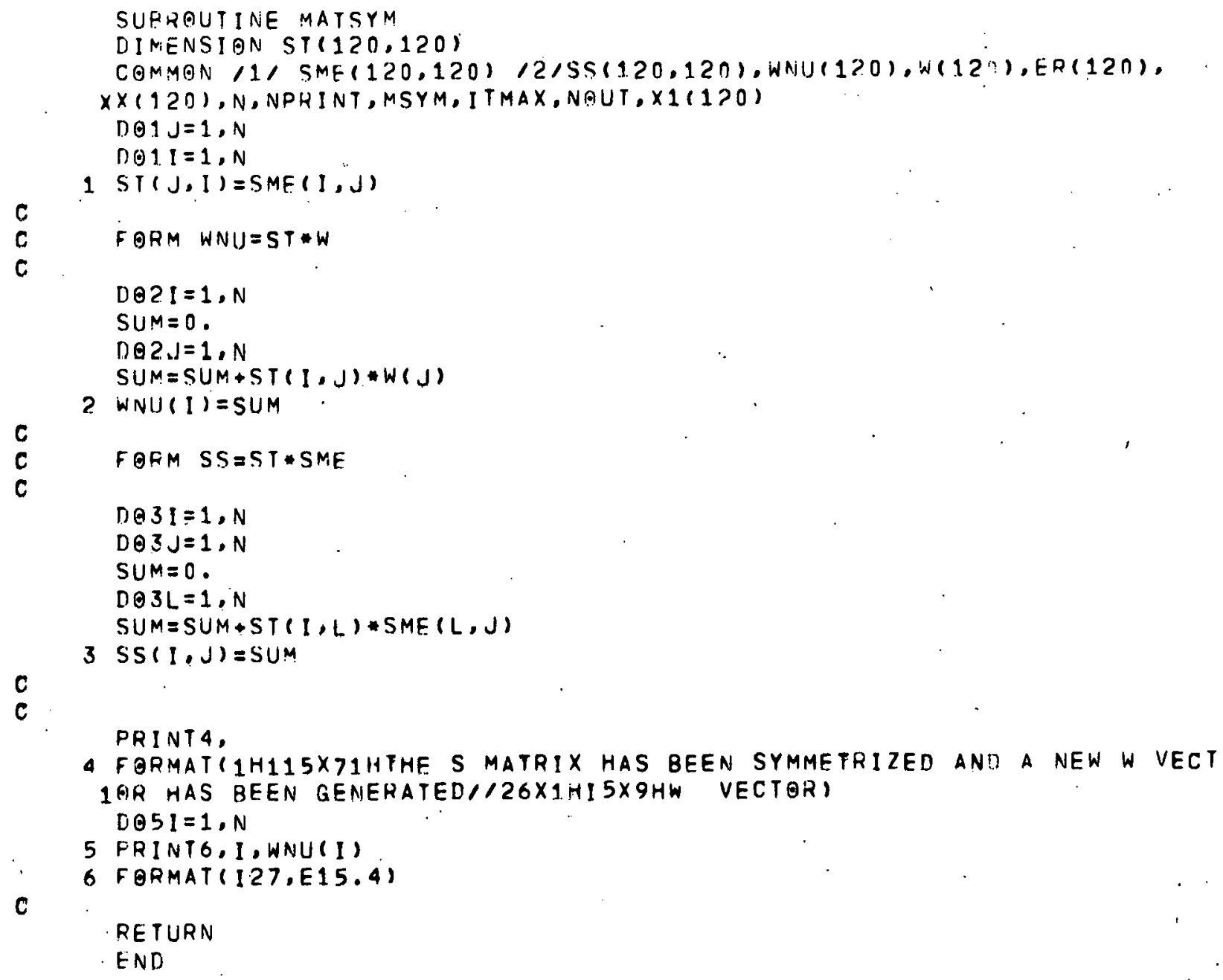

3. Printout of Subroutine MATl

SUBRQUTINE MATI

DIMENSION Y $(120), S(120)$

COMMON /1/ SME(120,120) /2/SS(120,120).WNU(120),W(12).,ER(120),

$X X(120), N, N P R I N T, M S Y M, I T M A X, N \theta U T, X 1(120)$

BANK, (MAT1), 121

c

READ1,N, M1, I SCALE, NPRINT, MSYM, MTYPE, ITMAX, NOUT, NERP

1 FORMAT 1216$)$

READ2, (Y $(1), 1=1, N)$

READ2, $(S(1), 1=1, N)$

READ2, $(\times 1(1), I=1, N)$

2 FORMAT (6F12.4)

GO IO $(20,30)$ NERR

20 READ 2, (ER $(1), 1=1, N)$

GO FO 50

30 READ 2, ERROR

DO $40 \quad I=1, N$

$40 E R(1)=E R R Q R$

50. CONPINUE 
$c$

c

PRINT3;

3 FORMATI IHI15X38HITERATIVE SOLUTION OF DETECTION SYSFEM//26X1HI7X5H 1S SET8X9HY VECTOR6X9HW VECFOR6X9HER VECTOR6X9HX1 VECTOR)

C

c

FORM W VECTORS

$D Q 4 I=1, N$

$4 W(I)=E X P F(S(I)) * S(1) * *(1-M 1) * Y(I)$

$D Q 5 I=1, N$

5 PRINT6,1,S(1),Y(1),W(1),ER(1):X1(1)

$c$

6 FORMAT $(127,5 E 15,4)$

C

REAU2,. $\quad A C F E R, X$

GOTOI11,7IISCALE

7 D०8!=1.N

$8 S(1)=S(I) * F A C T O R$

PRINT9,FACTOA

9 FORMAT 143 HITHE S MATRIX ELEMENTS HAVE BEEN SCALED BY F11,6,1/26X1H 1 I XX5HS SETBX9HY VECTOR6X9HW VECTORGX9HXI VECTORI

$D \theta 10 I=1, N$

$W(I)=E X P F(S(I)) * S(1) *(1-M I) * Y(I)$

10 PRINF6, $1, S(1), Y(1), W(1), X 1(1)$

11 CONPINUE

C

c

FORM S MATRIX

$D .013 !=1, N$

$D \theta 13 J=1, N$

$A=J$.

$A=A * X$

$B=A-1$.

$\operatorname{SME}(I, J)=S(1) * B$

GQTO\{12,13)MTY.PE

$12 Q=G A M M A(A)$

$\operatorname{SME}(I, J)=\operatorname{SME}(1, J) / Q$

13 CONTINUE

RETURN

END

4. Printout of Subroutine $\operatorname{MPRINT}(\mathrm{X}, \mathrm{N})$

SURROUTINEMPR INT $(X, N)$

c

DIMENSION X(120.120)

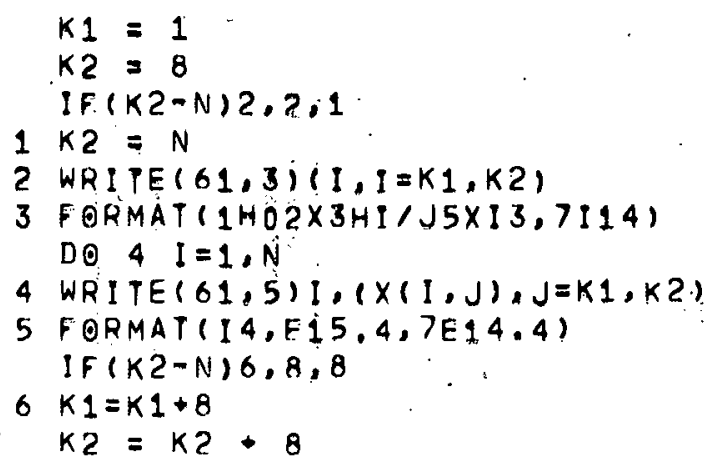




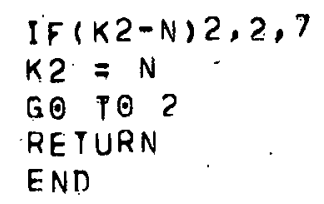

5. Printout of Subroutine MAT2

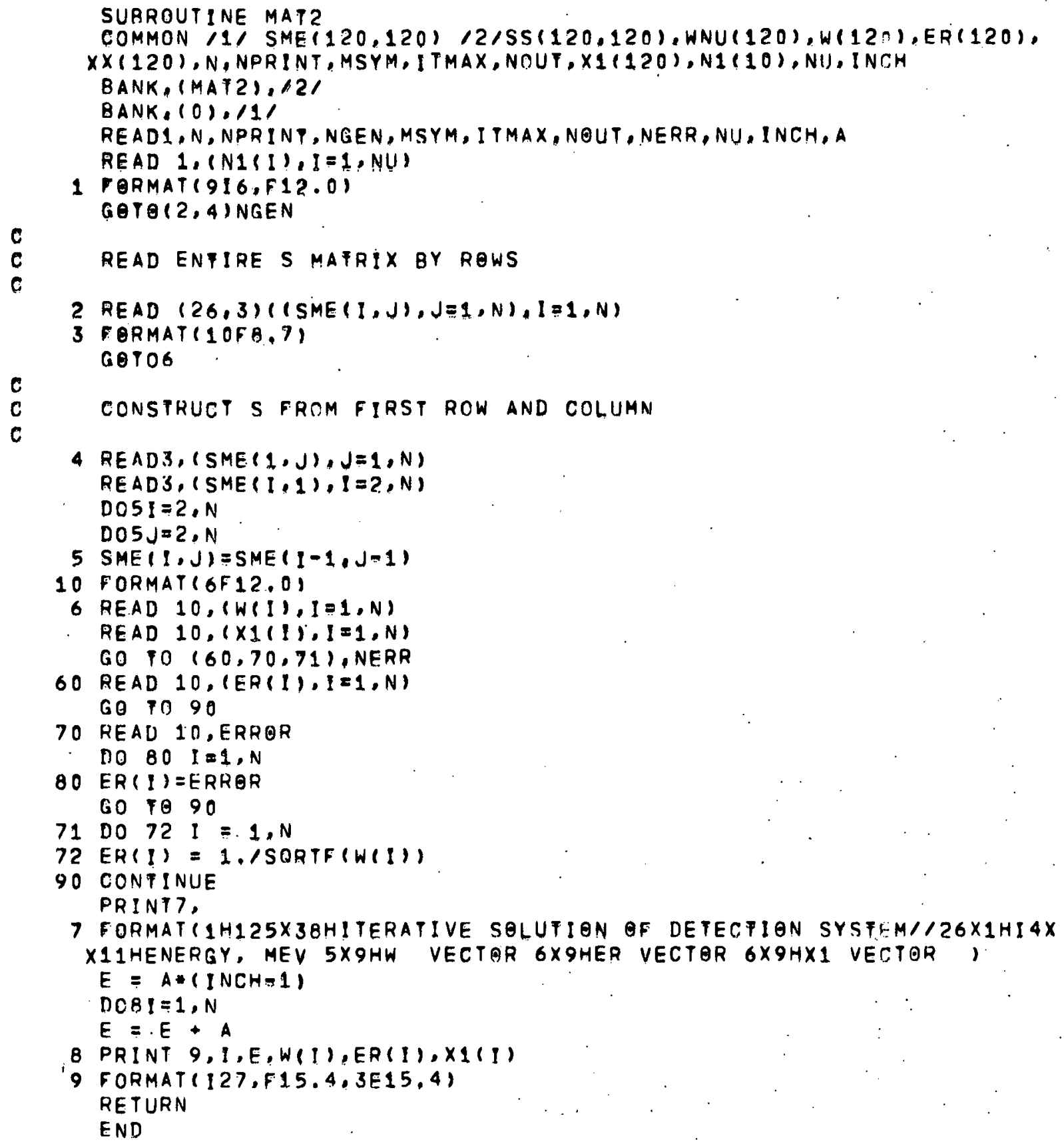


TABLE C.II. ITERATE Input

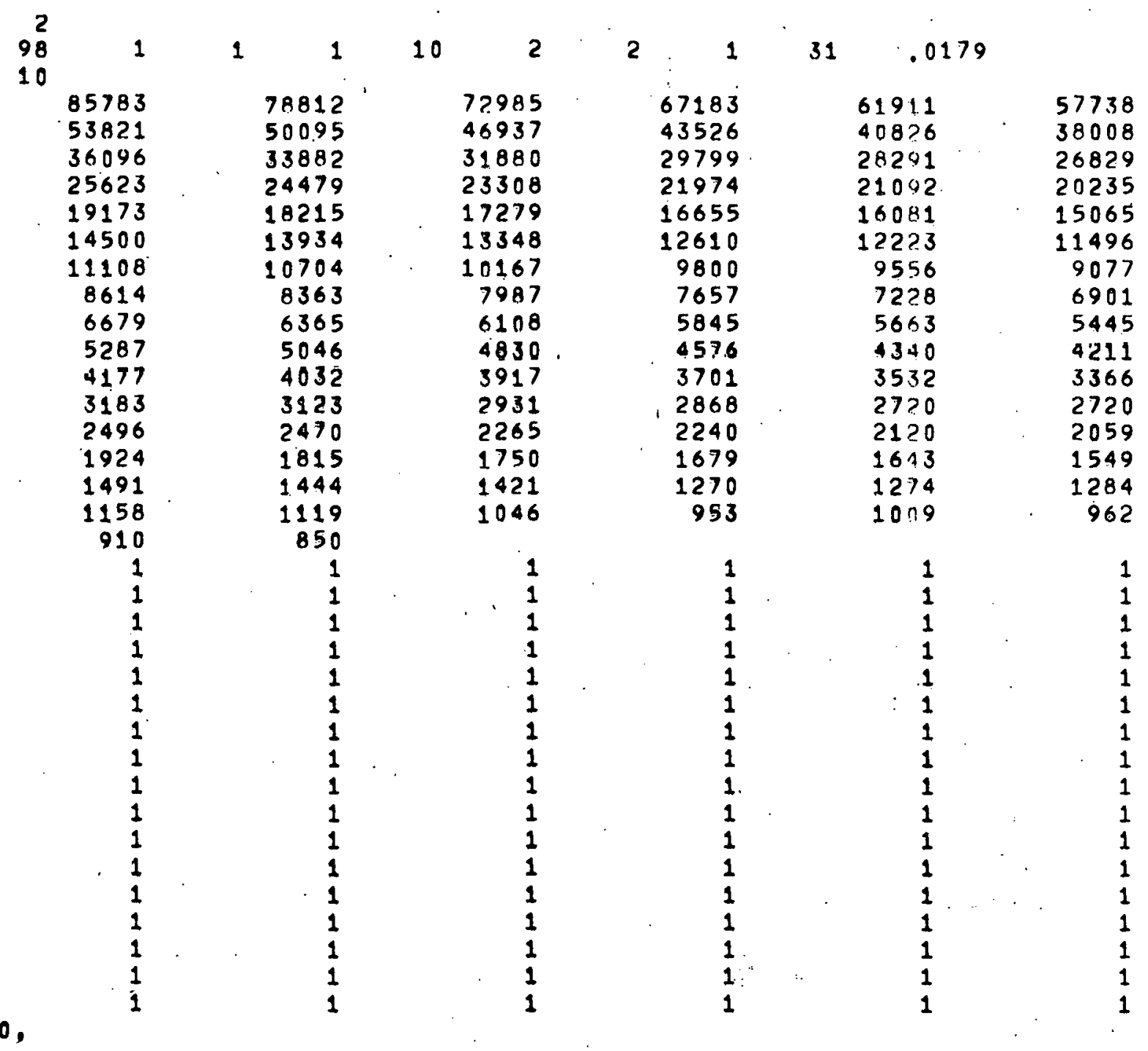




\section{APPENDIX D}

\section{PSNS Code and Flow Diagrams}

TAB LE D.I. PSNS Input

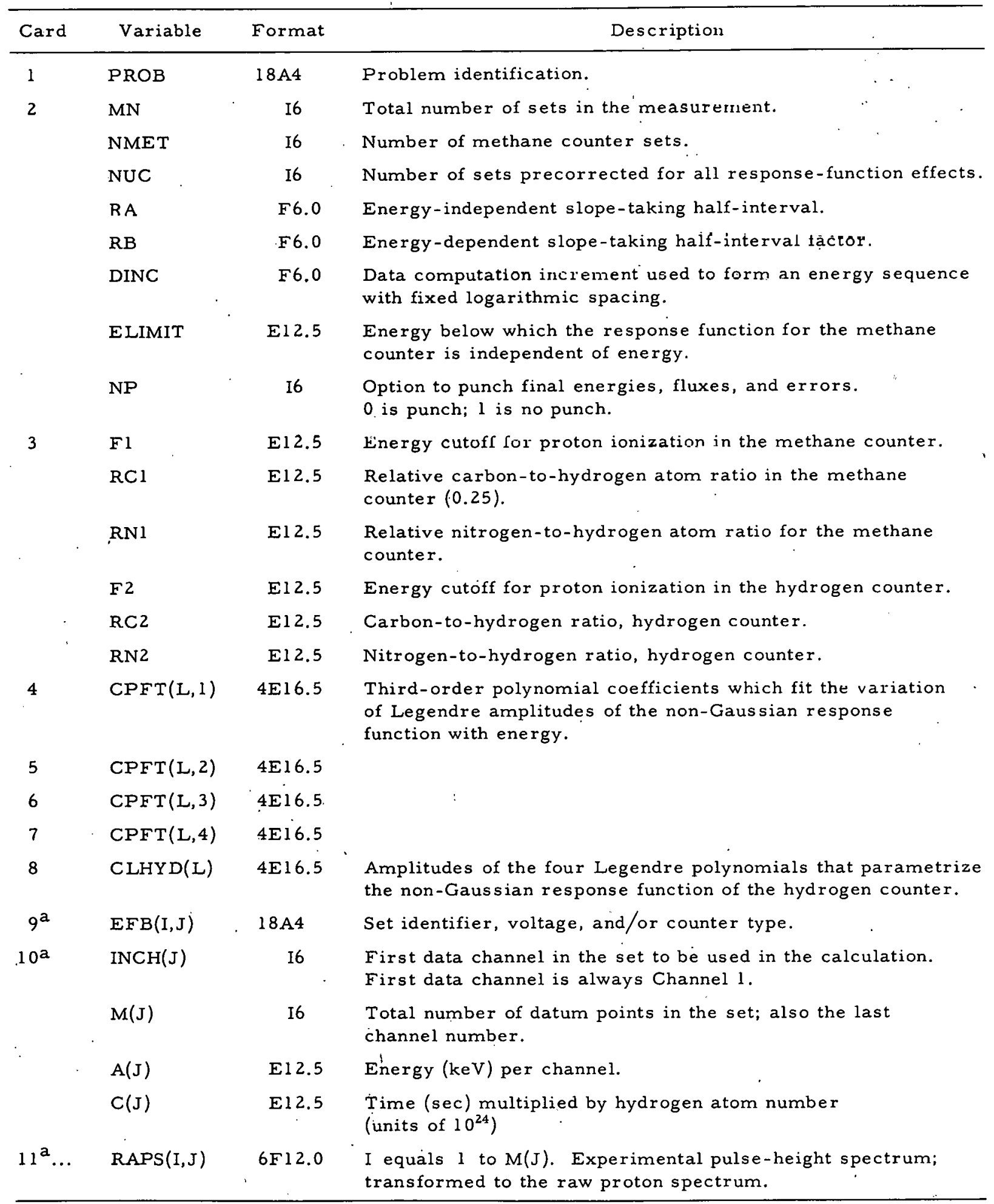

${ }^{a}$ Cards from 9 and on are used for additional sets up to MN. MN may be as high as 20 and the maximum $M(J)$ is 128 . 
TABLE D.II. Other Variables Used in PSNS

\begin{tabular}{|c|c|}
\hline Variable & Description \\
\hline $\operatorname{CHEN}(I, J)$ & $\begin{array}{l}A(J) \text { times the channel number plus the cutoff energy. CHEN is the energy (keV) } \\
\text { of each datum point. }\end{array}$ \\
\hline MNOLL(J) & $\begin{array}{l}\text { Minimum uonuverlapping limits for each set; used in establishing limits for } \\
\text { response-function integration. }\end{array}$ \\
\hline $\operatorname{PRAPS}(I, J)$ & Proton spectrum corrected for response-function effects. \\
\hline $\operatorname{EDEN}(I)$ & Energy points with-fixed logarithmic spacing within energy limits of the data. \\
\hline ERFL(I). & Absolute flux error (rms). \\
\hline $\operatorname{FLXL}(\mathrm{I}, \mathrm{I})$ & Corrected flux per lethargy using PRAPS(I,J). \\
\hline$F L X L(2 . I)$ & Raw flux per lethargy uoing $R \wedge \Gamma G(I, J)$. \\
\hline $\operatorname{HPCOR}(1)$ & Fractional correction to $F L X L(1, I)$ from recoil events involving carbon and nitrogen. \\
\hline CLEG(I) & $\begin{array}{l}\text { Amplitudes of corresponding Legendre polynomials. These amplitudes are energy- } \\
\text { dependent for the methane counter but not for the hydrogen counter. }\end{array}$ \\
\hline PLEG(I) & Legendre polynomials defined in the interval 0 to 1 . Only the first four are used. \\
\hline
\end{tabular}

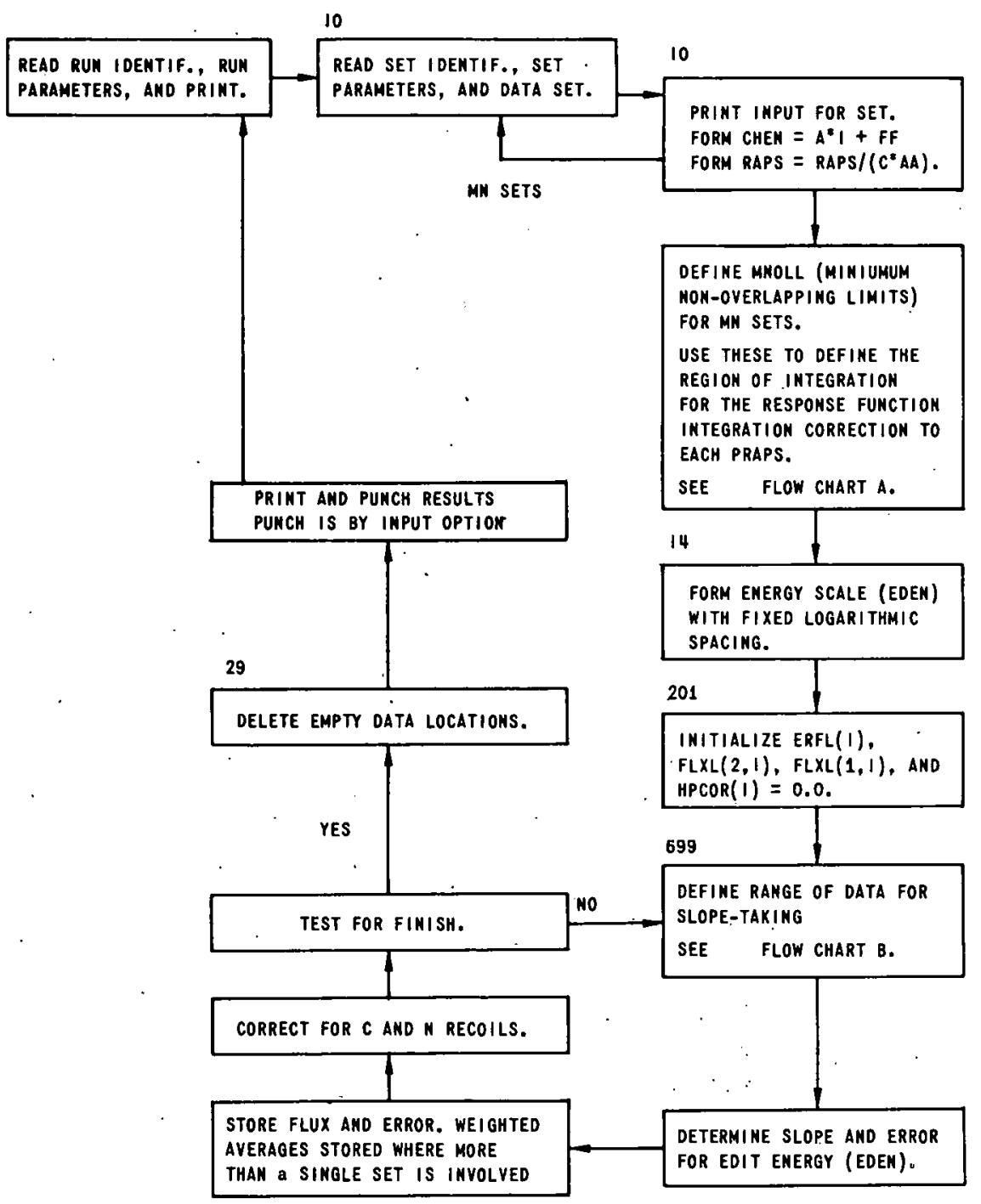




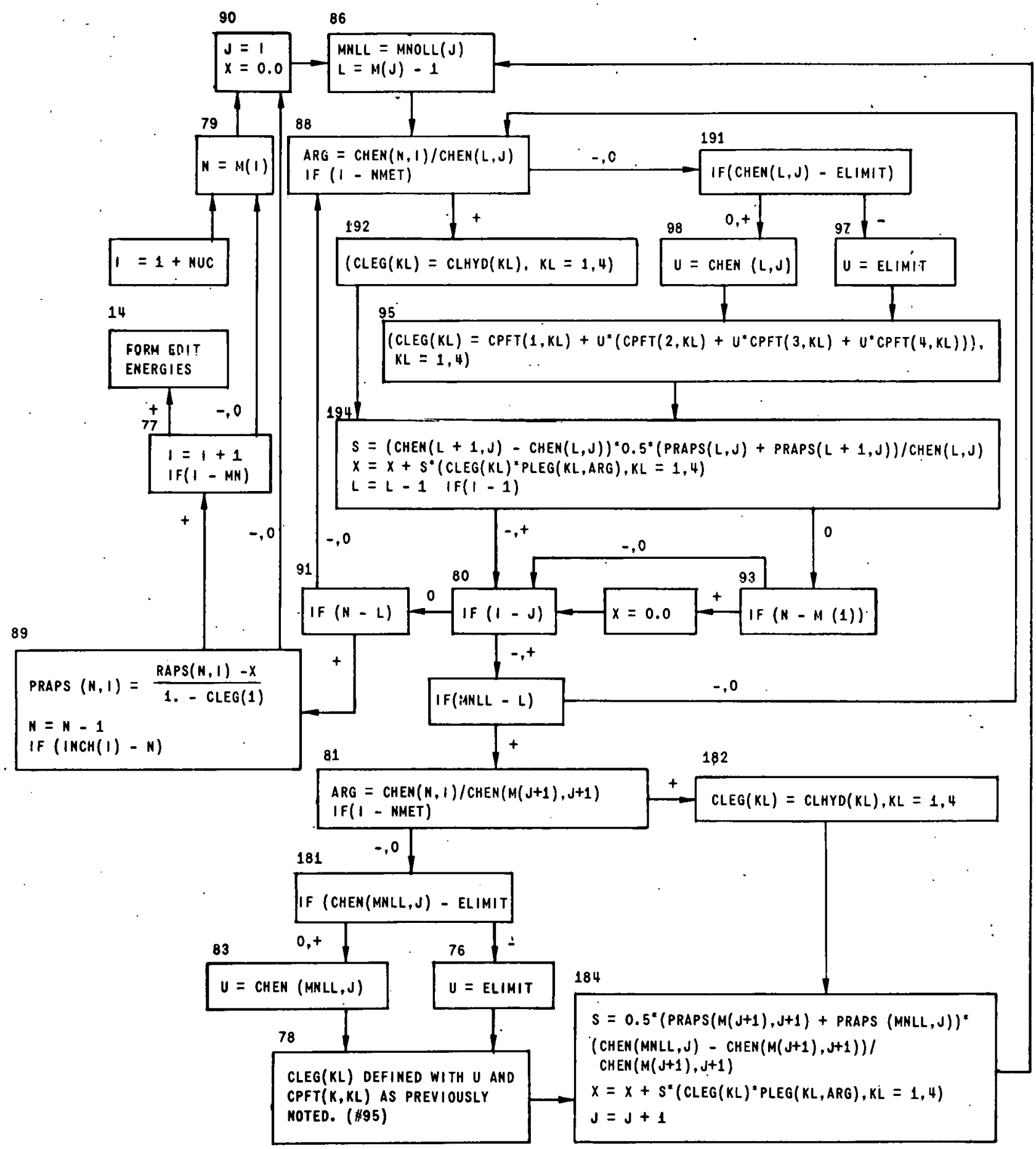

112-9121 Rev. 1

Fig. 36. PSNS. Flow Chart A for Response-function Integration 


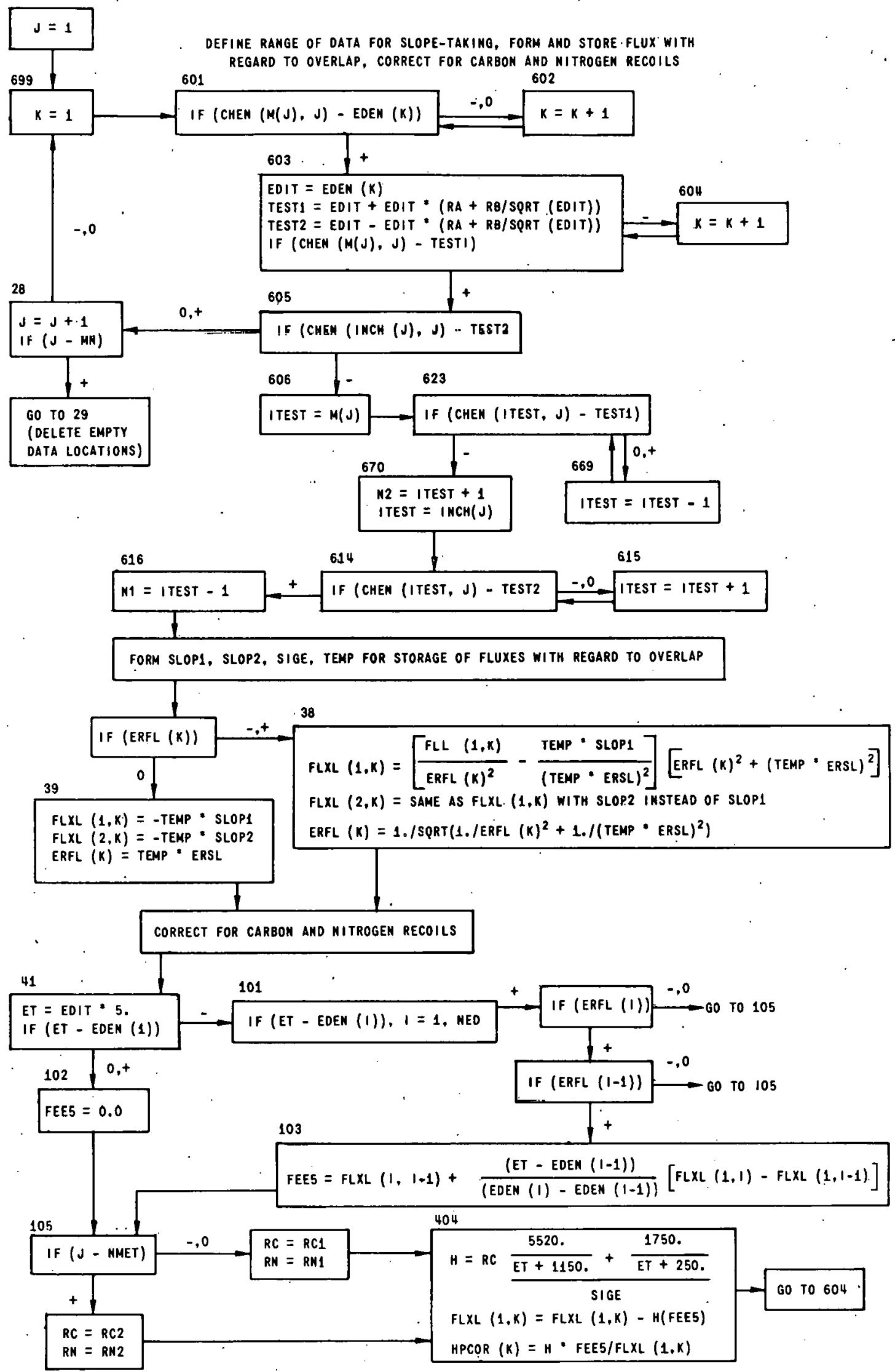

Fig. 37. PSNS Flow Chart B for Slope-forming Routine 
1. Printout for Program PSNS

PREGRAM PSNS

REVISION OF AUGUST 1967

PROTON SPECTRUM TO NEUTRON SPECTRUM

DIMENSION RAPS $(128,20)$, PRAPS $(128,20)$, CHEN $(128,20), E F R(20,18)$, PROB

$X(18), I N C H(20), M(20), A(20), E(20), F(20), R C(20), R N(20), K P C Q R(500)$, $X F L X L(2,500)$, ERFL $(500)$, EDEN $(500), M N Q L L(20), C L H Y D(6), C P F T(6,6)$

COMMON FLXL,ERFL

$X$, CLEG(6)

4 FORMAT $(216,2 E 12,5)$

300 FORMAT ( $4 E 16.5)$

733 FORMAT (6512.4)

2 FORMAT (6E12,5)

55 FORMAT (N/16A4)

200 FORMATIIH $16, E 17,5, E 18,5, E 20,5)$

58 FORMAT $(316,376,0, E 12,5,16)$

5 FORMAT (18A4)

100 FORMAT(G//),3X3IHPROGRAM OUFPUT. IS LISTED RELOW. 4( 1$), 3 \times 8 H C H A N$,

299 FORMAT $(31 / 1,3 \times 46$ HTHE SPECTRUM COMPUTATION INCREMENT WAS CHOSEN

59 FORMAT16F12.01

$X / 3 \times 38$ BHHE SLOPE =TAKING HALF INTERVAL USED IS F6,3,2X 4HPLUS X5BHCHANNEL ENERGY RAW PRETON DIST, CORRECTED PROTON DIST, 11 $X 4(1), 3 \times 23 H A$ W CUT-OFF ENERGY OF F6,3, $2 \times 20 H K E V$ FOR THE METHANE $X / 3 X$ GHTO BE F6,3,2X15HLETHARGY UNITS. $1 / 1$

$X, 3 \times 14$ HBEEN ASSUMED.

XF8.3,2X53HTIMES FHE RECIPROCAL SOUARE ROBT OF ENERGY (IN KEV), $x, 3 X$ 5HWAS $6.4,2 X 41$ HAND PHE RAYIO OF NITROGEN TO HYDROGEN WAS $X 3(1), 3 \times 54 H T H E$ RATIO OF CARBON TO HYDROGEN IN THE METHANE COUNTER $X / 3 \times F 6,4,1 \times 45 \mathrm{H}$. THESE RATIOS FOR THE HYDROGEN COUNTER WERE F6,4, 400 EORMAT (3/ $/ 1,2 X 1$ IHN2X13HRAW FLUX/LTY.2X11HEDIF ENERGY $3 X$ 9HFLUX/LTY. $X, 3 \times$ 5HAND $6.4,2 \times 13$ HRESPECTIVELY,

500 FORMAT $18 \mathrm{H}$. INITIAL CHANNEL = $14,10 \times 15 \mathrm{HFINAL}$ CHANNEL $=14.01(10 \mathrm{~F} 7.0) 1$ $\times 3 \times 1$ OHFLUX ERRQR $2 \times 12$ HHEAVY RECOIL// (14,5E13,4)

501 FORMAT ( $1 / / 1,1$ H $11 \times 28$ HAIENERGY(KEV) PER CHANNEL) = 18XE12.3.11H $11 \times$ X4GHC(TIME(SEC) X HOATOM NO, (10 TO PHE 24)) =E12.3.1,

21 FORMAT(1H1 $10 \times 2 B H A N L=R P 393 \times$ (PSNS) AUG, 1967 ) $X, 3 \times 13$ HCOUNTER AND F6,3, $2 \times 33$ HKEV FOR THE HYDROGEN COUNTER HAS $X$ I 3 X56HPROPOR IONAL COUNTERS, VARIOUS CORRECTIONS ARE APPLIED

22 FORMATI3(/),3X 48HPROGRAM TO PROCESS PROFON RECOIL DATA FROM

$X, 3 X 46 H I N C I D E N T$ NEUTRON FLUX. SPECTRUM IS DERIVED. $X$; $X 56$ HTO REMOVE NON-IDEAL COUNTER RESPONSE EFFECTS AND THE

23 FORMAT $(3(1), 3 \times 2 O H T H I S$ PROBLEM IS FOR,

24 FORMATI3 $1 / 1,3 \times 11$ HA TOTAL OF $14,2 \times 29$ HSETS OF DATA (INCLUDING BOTH $X>3 \times 58 H V O L$ Q $A G E$. AND COUNTER CHANGES) WERE ACCUMULATED. FAR THIS $X 13 \times 22$ HEXPERIMENT. THE FIRST $14,2 \times 28$ OHSETS REFER FO FHE PREDOMINAT $X / 3 X 52 H N T L Y$ METHANE COUNTER COVERING THE HIGHER ENERGIES, 25 FORMAT (3 $111,3 \times$ 14,2X41HSETS IIN ORDER OF DECREASING ENERGY) HAVE $X \mid 3 \times 55$ HBEEN PRE GCORRECTED FOR ALL NON-IDEAL RESPONSE EFEEC IS, ?

26 FORMAT(3/1),3X47HTHE ENERGY LIMIT FOR THE METHANE COIJNTER BELOW $X / 3 \times 57 \mathrm{HWHICH}$ THE RESPONSE FUNCPION IS NOT DEPENDENT UPON ENERGY $X / 3 X$ 4HIS E12,5,2X42HKEV. THE PREDQMINANTLY HYDROGEN COUNTER IS $X / 3 X \bar{X} 58 H N O T$ USED AT ENERGIES SUFFICIENTLY HIGH TO PERMIT THE. $X / 3 X 3$ THRESPONSE FO BECOME ENERGY DEPENDEN $T, 1$

27 FORMAT $131 \%, 3 \times 47$ THHE FOUR POLYNOMIAL TERMS USED TO EXPRESS THE $X / 3 \times 57$ HENERGY DEPENDENCE OF THE AMPLITUDE OF THE FOUR LEGENDRE $X 13 \times 57 H F U N C P$ IONS WHICH PARAMETRIZE PHE ENERGY DEPENDENT RESPONSE

$X / 3 \times 3$ THFUNCTION FOR THE METHANE COUNTER ARE, 1 
16 FORMAT(3//),3X47HTHE FOUR LEGENDRE FUNCTION AMPLITUDFS USED TO $X / 3 \times 57 H P A R A M E T R I Z E$ THE RESPONSE FUNCTION FOR THE PREDOMINANTLY $X / 3 \times 27$ HHYDROGEN FILLED CQUNPER ARE ',

17 FORMAT $(6(1), 3 \times 33$ HA SUMMARY OF INPUT DATA FQLLOWS. 1 ,

35 READ $5,(P R O B(1), 1=1,16)$

IF IEOF, 60136,37

37 READ 5B, MN, NMET, NUC, RA, RB, DINC,ELIMI F, NP

READ 2,F1,RC1,RN1,F2,RC2,RN2

READ $300,((C P F Y(L, K), L=1,4), K=1,4)$

READ $300,(C L H Y D(L), L=1,4)$

PRINT 21

PRINT 22

PRINT 23

PRINT 5, (PROB (I), $(=1,18)$

PRINT 24, MN.NMET

PRINT 25, NUC

HKINT 299, DINC,RA, RB, F1,F2,RC1,RN1, RC2,RN2

PRINT 26,ELIMIT

PRINT 27

PRINF $300,((C P F T(L, K), L=1,4), K=1,4)$

PRINT 16

PRINT $300, C$ CLHYD $(L), L=1,4)$

PRINT 17

DO $10 \quad J=1, M N$

READ $5,(E F B(J, K), K=1,18)$

PRINT $55,(E F B(J, K), K=1,18)$

READ 4,INCH(J),M(J),A(J),C(J)

PRINT 501,A(J),C(J)

MAXCHAN = M(J)

INCHAN = INCH(J)

READ 59, (RAPS $(1, J), 1=1$, MAXCHAN)

PRINT 500, INCH(J),M(J), IRAPS (I,J), I INCHAN,MAXCHAN)

c

c

c

FORM CHANNEL ENERGY AND PROTON ENERGY DISTRIBUFION

$A A=A(J)$

IF(J-NMEF) 409,409,411

409 FFIF 1

$G \theta$ TO 412

$411 F F=F 2$

412 DQ 10 I I, MAXCHAN

CHEN $(1, J)=A A+!$ \&F

10 RAPS $(1, J)=1.0 /(C(J)-A A) \cdot \operatorname{RAPS}(1,4)$

C DEFINE MINIMUM NON-OVERLAPPING LIMITS (MNOLL(J)) FOR EACH SET

0

$J=1$

$63 \mathrm{~K}=1 \mathrm{NCH}(\mathrm{J})$

IF (J०I-MN) 40,40.62

40 IF (CHEN(K, J)=CHEN(M(J+1),J $\$ 1) 167,68,68$

$67 K=K+1$ IF(K-M(J)/40.68.68

68 MNOLL $(J)=K$

$J=J+1$

GO $P \theta \quad 63$

$c$

62 MNOLL(MN) INCH(MN)

C RESPONSE FUNCTION INTEGRATION FOR BOTH COUNTERS ALLOWING ENERGY

C DEPENDENCE ON PHE METHANE CQUNTER AT HIGHER ENERGIES 


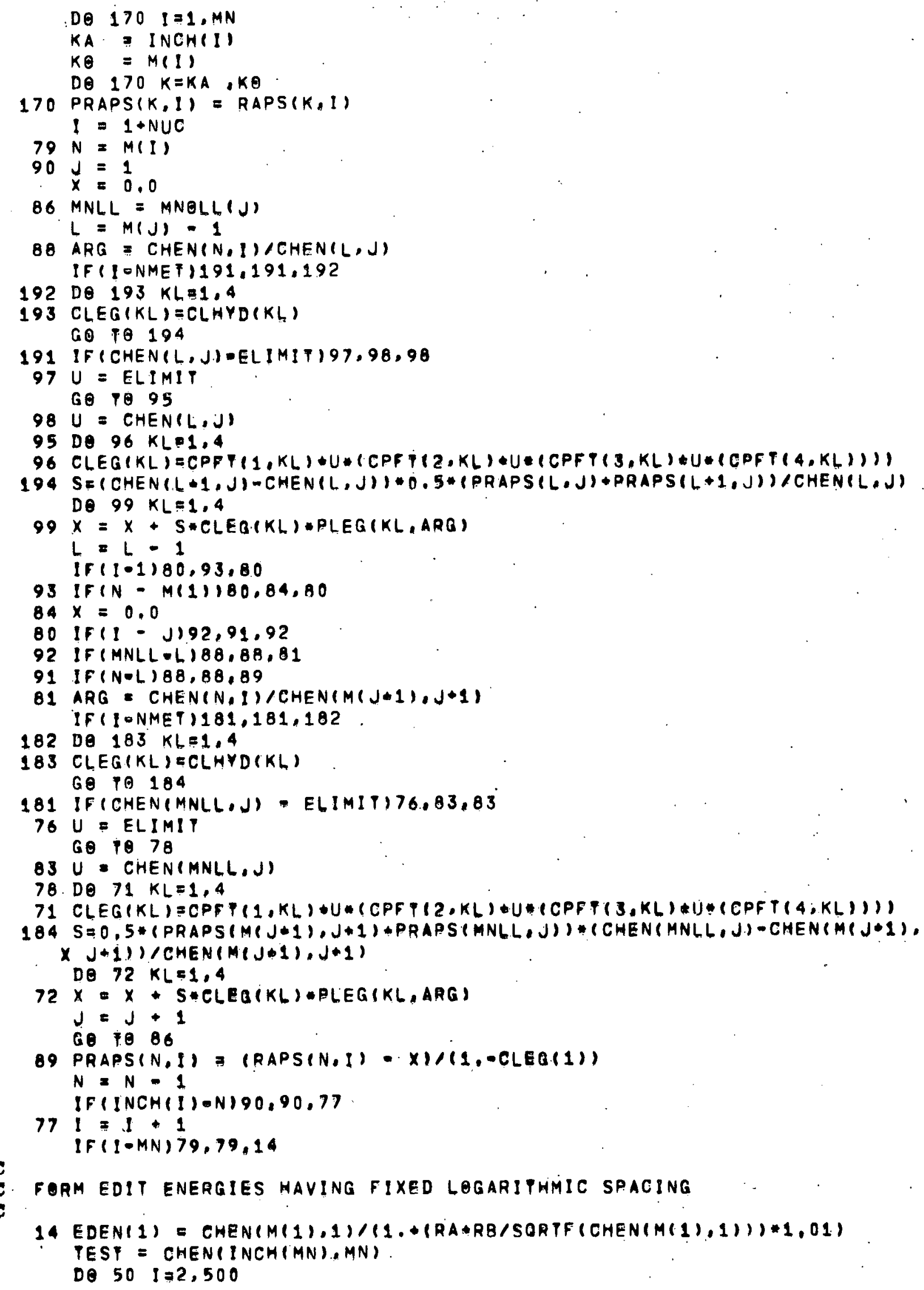




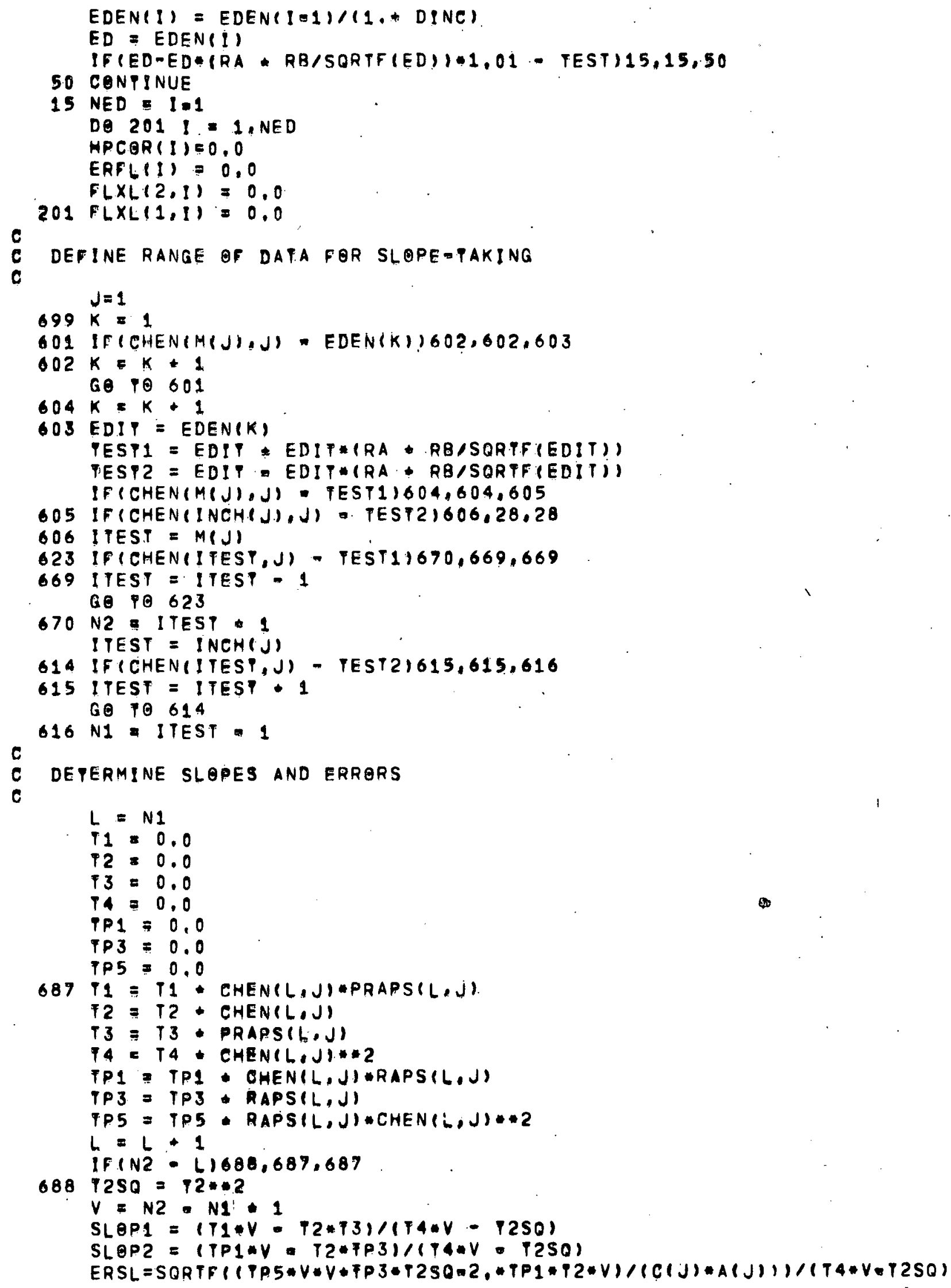




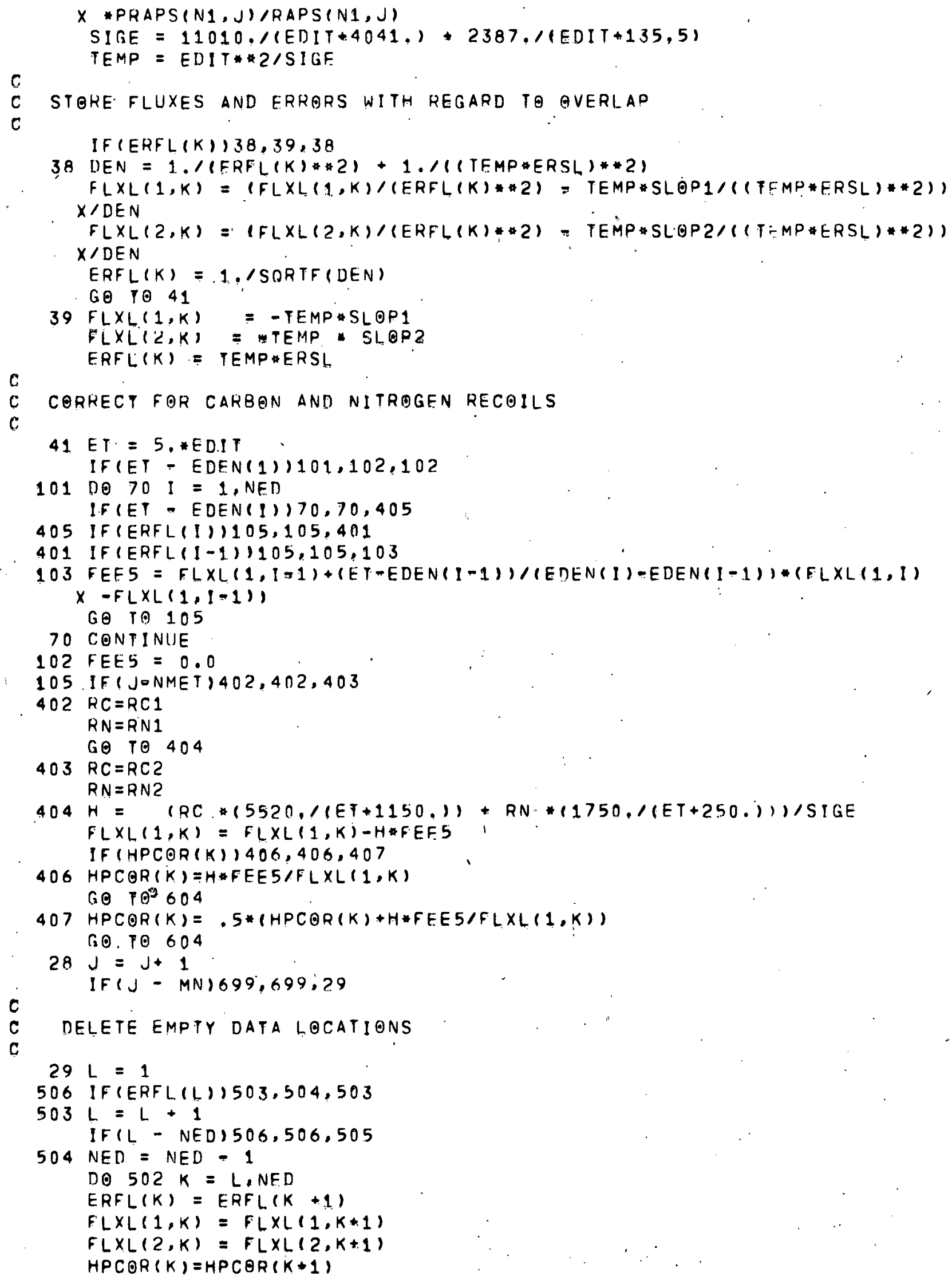




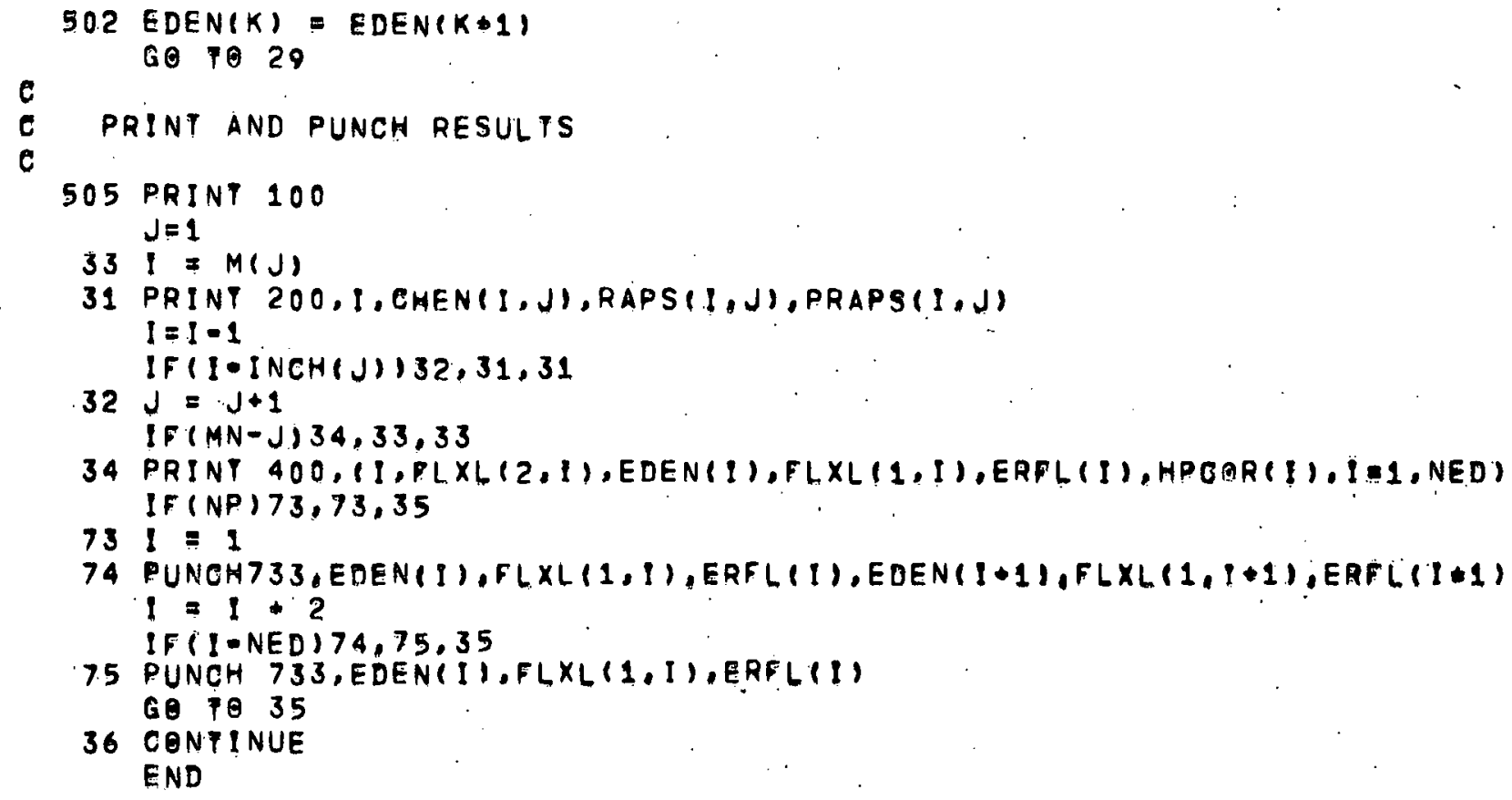

2. Printout for Function PLEG(N,ARG)

FUNCTION PLEQ(N,ARG)

$G O F \theta(1,2,3,4), N$

1 PLEQ $=1$. RETURN

2 PLEQ=12.AARG - 1.1.1.73205

RETURN

3 PLEQD16.*ARG:1ARG-1.1*1.1*2.23607 RETURN

4 PLEG $=(-1,+A R G *(12,-A R G *(30,-A R G * 20.1)) * 2.64575$

RETURN

END

3. Printout of PSNS Output

$$
A N L=R P 393 \times \text { (PSNS) AUG, 1967 }
$$

PROGRAM TO PROCESS PROTON RECOIL DATA FROM

PROPORTIONAL COUNTERS. VARIOUS CORRECTIONS ARE APPLIED

FO REMOVE NON-IDEAL COUNTER RESPONSE EFFECTS AND THE

INCIDEN NEUTRON FLUX SPECTRUM IS DERIVED.

PHIS PRORLEM IS FOR

PSNS TEST PROBLEM: TYPICAL FAST REACTER SPECTRUM 
A TOTAL OF 11 SETS OF DATA IINCLUDING BOTH VOLTAGE AND COUNTER CHANGESI WERE ACCUMULATED FOR THIS EXPERIMENT. THE FIRSF 3 SETS REFER TO THE PREDOMINA: NTLY METHANE COUNTER CQVERING THE HIGHER ENERGIES.

1 SETS I IN ORDER OF DECREASING ENERGYI HAVE BEEN PREOCORRECTED FAR ALL NON-IDEAL RESPONSE EFFECTS.

THE SPECTRUM COMPUTAFION INCREMENT WAS CHOSEN TO BE 0.050 LETHARGY UNITS.

FHE SLQPE-TAKING HALE INTERVAL USED IS 0.050 PLUS

0.150 TIMES THE RECIPROCAL SOUARE ROQT OF ENERGY (IN KEV),

A W CUT-OFF ENERGY OF 4,000. KEV FOR PHE MEFHANE COUNTER AND O.290 KEV FOF THE HYDROGEN COUNTER HAS BEEN ASSUMED.

PHE RATIO AF CARBON PO HYDRQGEN IN THE METHANE COUNTER WAS 0.?5.00 AND THE RAPIO OF NITROGEN FO HYDROGEN WAS 0.0100 . THESE RAPIOS FOR THE HYDROGEN COUNTER HERE 0.0250 AND U.0250 RESPECFIVELY,

THE ENERGY LIMIP FOK THE METHANE COUNTER BELOW

WHICH THE RESPONSE FUNETION IS NOT DEPENDENT UPON ENERGY IS 5.52800E O? KEV, THE PREDOMINANTLY HYDROGEN COUNTER IS NOT USED AT ENERGIES SUFFICIENTLY HIGH FG PERMIP THE RESPONSE. TO BECGME ENERGY DEPENDENT.

FHE FOUR PALYNOMIAL FERMS USED TO EXPRESS THE

ENERGY DEPENDENCE OF THE AMPLITUDE OF THE FOUR LEGENDRE

FUNCTIONS WHICH PARAMETRIZE THE ENERGY DEPENDEN RESPONSE FUNCTION FOR THE MEPHANE COUNTER ARE

\begin{tabular}{|c|c|c|c|c|}
\hline $\begin{array}{r}-0.25384 E- \\
0.29185 E \\
-0.20740 E \\
-0.23164 E\end{array}$ & $\begin{array}{ll}01 \\
0 & 0 \\
0 & 0 \\
0 & 0\end{array}$ & $\begin{array}{r}0.58488 E-03 \\
-0.14058 E-03 \\
0.59950 E=03 \\
0.42971 E-03\end{array}$ & $\begin{array}{l}-0.18894 E-06 \\
=0.11469 E-06 \\
=0.38492 E-06 \\
=0.25025 E-06\end{array}$ & $466 E-10$ \\
\hline
\end{tabular}

THE FEUR LEGENDRE FUNCTION AMPLITUDES USED TO

PARAMETRIZE THE RESPONSE FUNCTION FQR THE PREDOMINANTLY. HYDROGEN FILLED COUNTER ARE

$0.87000 F-01 \quad-0.12900 E-01 \quad-0.69100 E=01$ 
TABLE D.III. PSNS Input Data

CH4 COUNTER AT $3200 \mathrm{~V}$, FEST PRQBLEM

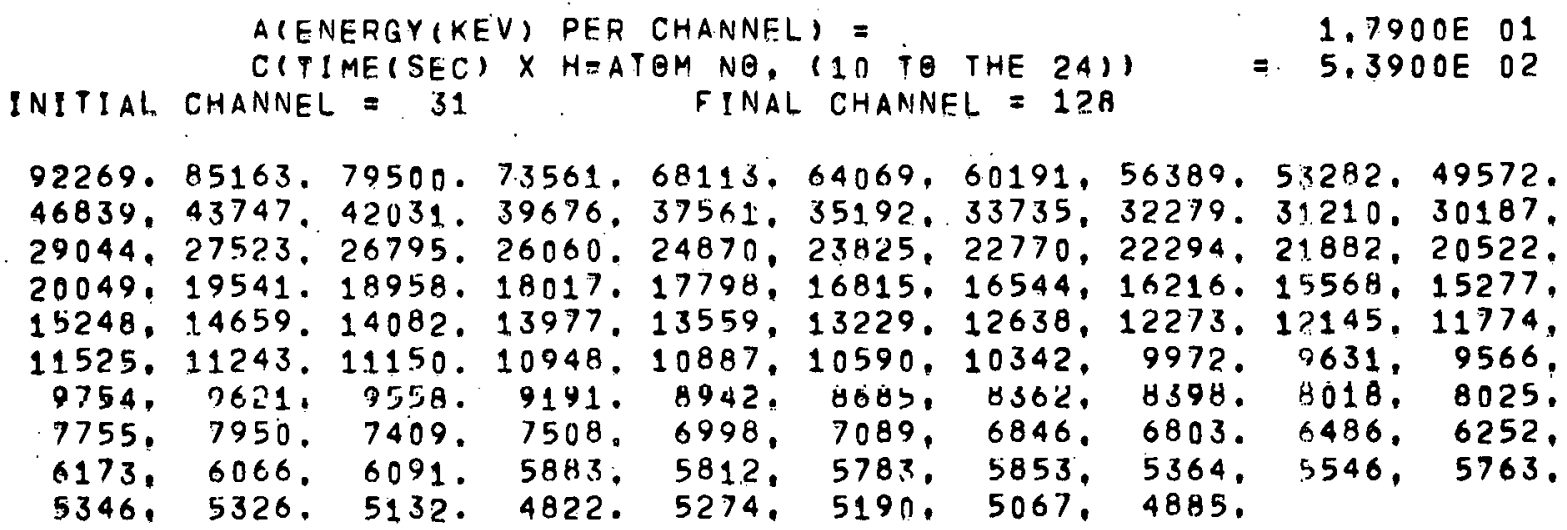

CHA COUNTER AT $3500 \mathrm{~V}$, FEST PROBLEM

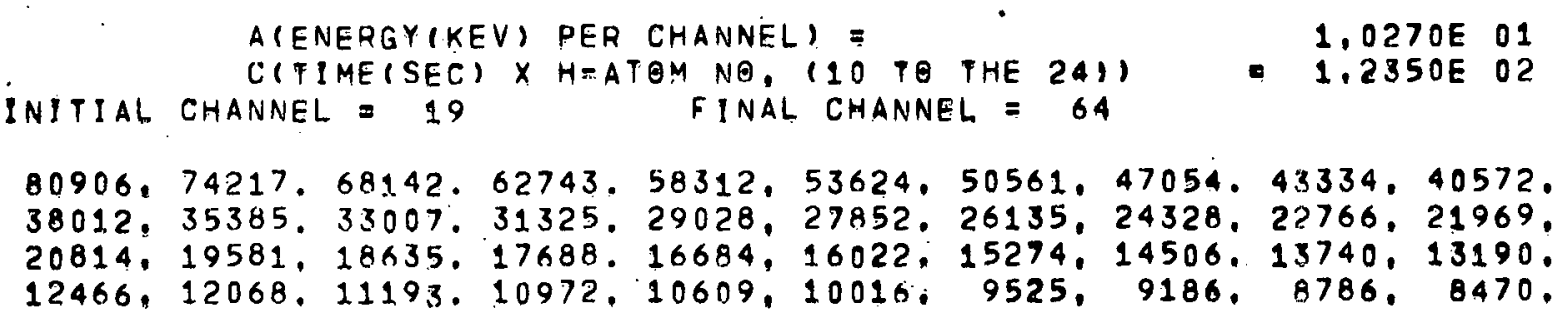
$8044,7704,7410,7192,6772,6485$,

CHA COUNTER AT. $3750 \mathrm{~V}$, FEST PROBLEM

A (ENERGY (KEV) PER CHANNEL) =

CIFIME(SEC) X HEATOM NO, (1O TO THE 24$)$ )
INITIAL CHANNEL $=19$
FINAL CHANNEL $=64$

$5.3500 E \cdot 00$

- $1,3300 E$ O2

\begin{abstract}
$118315,110072.101906,94413,87902,81725,76573,72758,68772,64953$, $61714,58923,55367,52384,49885,47327,45636,43408,41651,39930$. $38487,36862,35214,33892,32520,31105,29951,29038,27811.26999$. $25555,24802,23846,22970,22149,21769,20873,20126,19439,18888$, 18092 . 17547. 16911. 16639.15948,15280.
\end{abstract}

H2 COUNPER AT $3250 \mathrm{~V}$, TEST PROBLEM

$$
\begin{aligned}
& \text { A. (ENER(SY (KFV) PER CHANNEL) = } \\
& \text { C(TIME(SEC) X HEATOM NO, (10 TO THE 24)) = } 8,7400 E \text { O }
\end{aligned}
$$

INITIAL CHANNEL $=19$ FINAL CHANNEL $=60$

$94804,90134,85301,81014,76850,73415,70615,68100,64561,62370$. 59590 . 56992. 5521\%, 52509, 50589, 48866,46669,44850,43409,41784. $40334,38914,37687.36856 .34984,34054,33057,32154,31595,30578$. $29428.28676 .27741 .26371 .26022,25196,24324,23886.23130 .22080$. 22019.21296. 
TABLE D.III. (Contd.)

H2 COUNTER AP $3400 \mathrm{~V}$, TEST PROBLEM

A (ENERGY (KFV) PER CHANNEL) =

C(TIME (SEC) X HEATOM NO. (IO TO THE 241 )

$1,2900 E 00$

INITIAL CHANNEL $=19$

FINAL CHANNEL $=60$

$=8,6800 \mathrm{E} 01$

\begin{abstract}
$88423,84817,79706,75763,73483,71452,69845,68220,65525,63663$. $61611,59939,58159,56028,54473,53373,51031,50120,47898,46861$, $45041,44294,43051.42454,41000,40475,39477,38365,37236,36070$. 34956,34609 , 33384, 32731. 31953, 30418, 29704, 29368, 29230, 27632 . 26896,26438 .
\end{abstract}

H2 COUNFER AT 3550V. TEST PROBLEM

A (ENERGY (KEV) PER CHANNEL) = $7.4800 E-01$

C(FIME(SEC) X HEATOM NO. (10 TO THE 24)) $=9.6200 E 01$

INITIAL CHANNEL $=19$

FINAL CHANNEL $=60$

$88190,85373,81798,79652,77223,74833,72873,70799,69622,66736$, $64474,62818,61157.59283,57493,55614,53511,51916,50270,49118$, $484 \%, 47436,46592,46288,45882,44822,44656,43172,42436,42013$, $40810,40358,39164,38237,37655,36983,35633,34952,34416,33591$. 32810,32337 ,

H2 COUNTER AT $370 O V$, TEST PROBLEM

A(ENERGY (KEV) PER CHANNEL) =

C(PIME (SEC) X H=ATOM NO, (10 TO THE 24)1 = $8,5500 E 01$

INITIAL CHANNEL $=25$ FINAL CHANNEL $=60$

$57074,54944,53481,52084,50663,49361,48384,47248.46491,45385$. $43706,43329,47449,41648,41007,40391,39544,39186,38183,38120$. $36786.36595,35496.34852 .34389,34039,33216,32662.31997,31335$. 30.735 .29837 .29760 .28862 .27989 .27451$.

H2 COIJNTER AT 385OV. TEST PROBLEM

AlENERGY(KEV) PER CHANNEL) =

C(FIME(SEC) X HOATOM NO, (1D TO THE 24))

$2.4000 E=01$
$=\quad 1.1240 E 02$

INITIAL CHANNEL $=25$

FINAL CHANNEL $=60$

$58108,57186,56082,54853,53600,52749,51596,51319,49950,49826$. $49038,48183,47444,47144,46343,45404,44886,44331,43245,42985$, $42138,41327,40738,40291,39213,38735,38166,37665,37413,36133$, $35631,34982.34419 .33929,33697,32893$. 
TABLE D.III. (Contd.)

H2 COUNTER AT $4000 \mathrm{~V}$. TEST PRORLEM

A(ENERGY (KEV) PER CHANNEL) =

C(TIMF(SEC) X HIATOM NO. (10 TO THE 241$)$

$=\begin{aligned} & 1.3080 E-01 \\ & 1.1110 E-02\end{aligned}$

INITIAL CHANNEL $=25$

FINAL CHANNEL $=60$

$41082,40631,40145,39609,38862,38441,38217,37831,36851,36923$, $36300,35480,35395.35243,34775,34241,33855,33588,33497.33026$. $32378,32054,32339,31468,30762,30952,30488,30321.29906,29335$, $29001.28652 .28368 .2791 \hat{1} .27499,27158$.

H2 COUNTER AT $4150 \mathrm{~V}$. TEST PROBLEM

A(ENERGY (KEV) PER CHANNEL) =

CITIME (SEC) X H=ATOM NO. (10 TO THE 24) = $=1.0630 E=02$

INITIAL CHANNEL $=25$

FINAL CHANNEL $=60$

25821, 25824. 25244. 25266, 25017, 24657, 24659, 24382, 23789, 23865. $23701,23239,22964,23073,22476,22444,22174,22076,27003,22164$. 21674, 21576, ?1437. 21145.21483, 20890,20874,20659.20414,20233, 20275. 19986:19785.19602. 19291,1.9157.

H2 COUNTER AT $4300 \mathrm{~V}$, TEST PRORLEM

A(ENERGY (KEV) PER CHANNEL) = C (FIME (SEC) X H-ATOM NO, (10 TO THE 24))

$3,6900 E=02$

INITIAL CHANNEL $=25$ FINAL CHANNEL $=$. 60

18169, 17904, 17357. 17122. 17128, 17022, 16505, 16457.16129, 16275. $16205,16139.15847,16003,15593,15452,15267,15166,14943,14601$, $14943,14826,14614,14695,14427,14532,14028,14060,14075,14060$. $14068,13725,13775,13774.13272,13716$. 
TABLE D.IV. PSNS Output Data

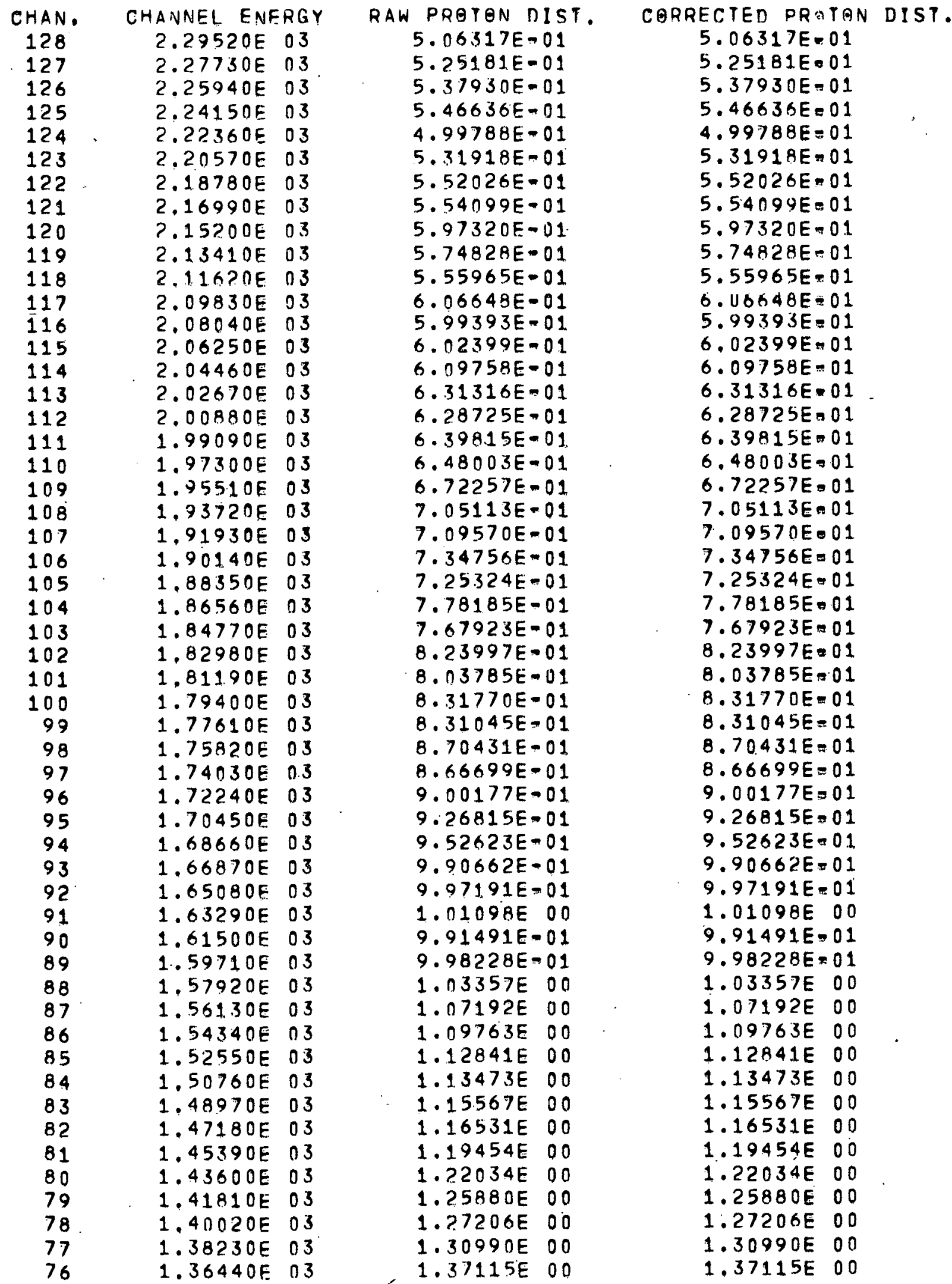


TABLE D.IV. (Contd.)

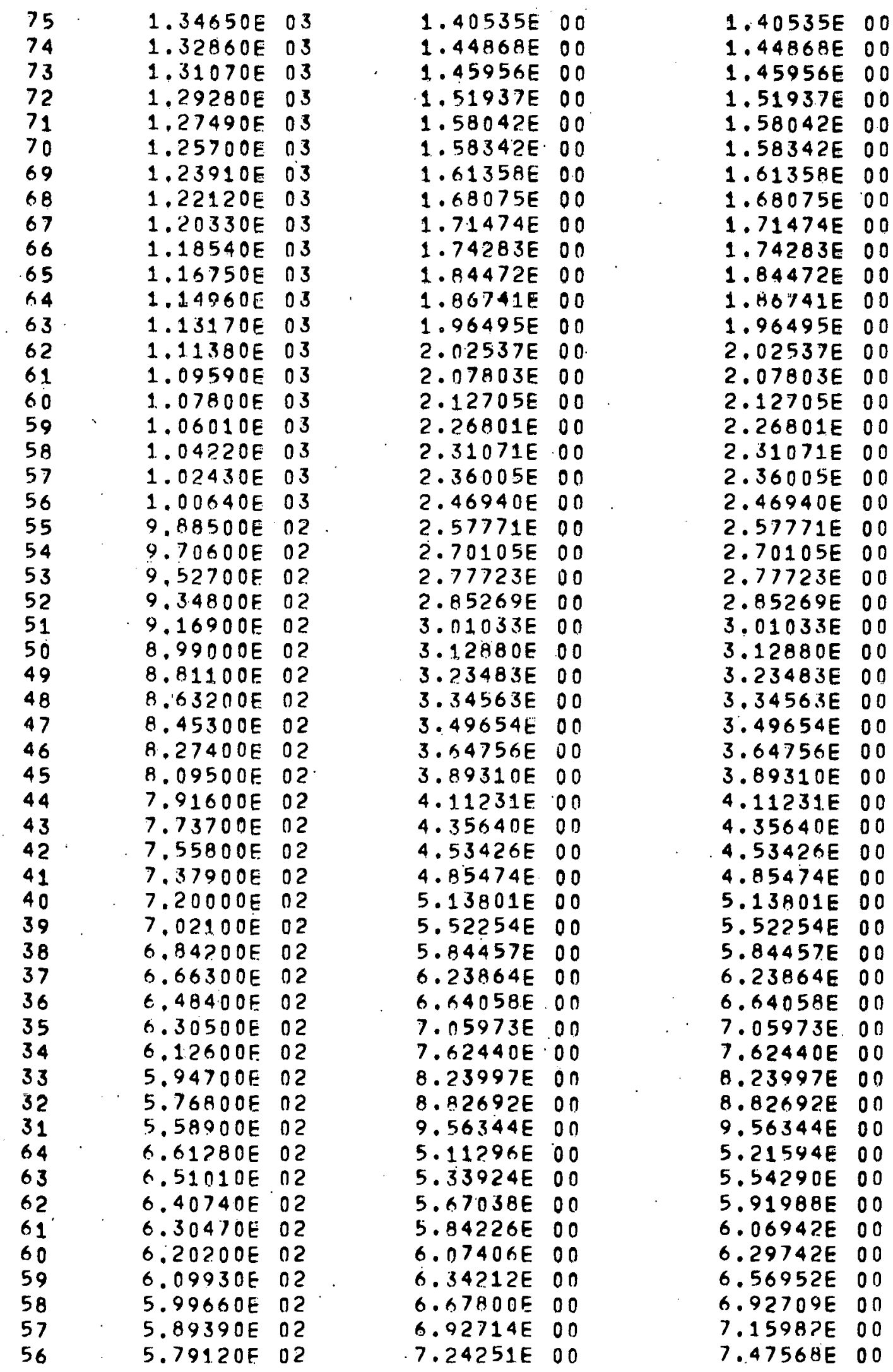


TABLE D.IV. (Contd.)

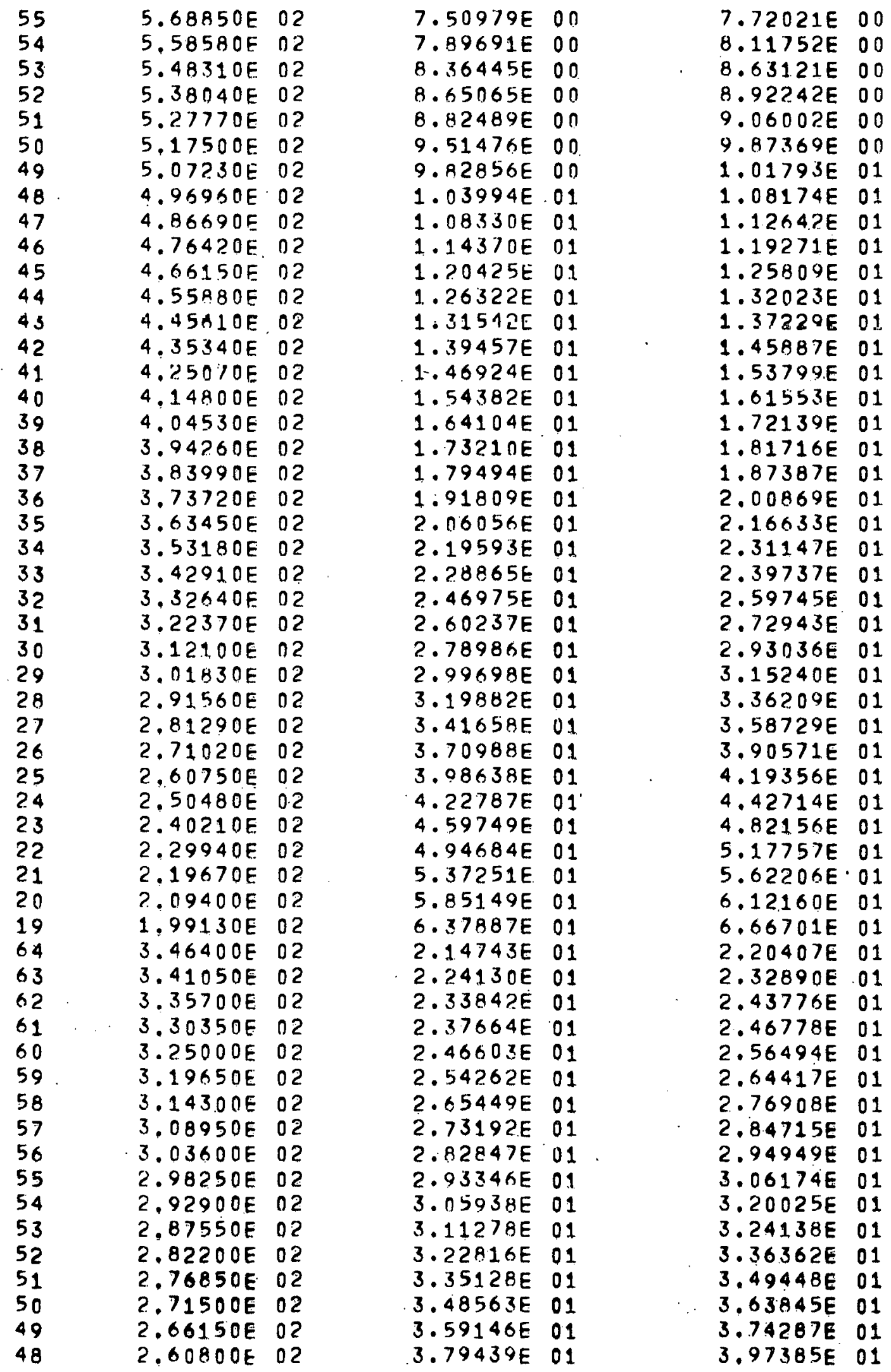


TABLE D.IV. (Contd.)

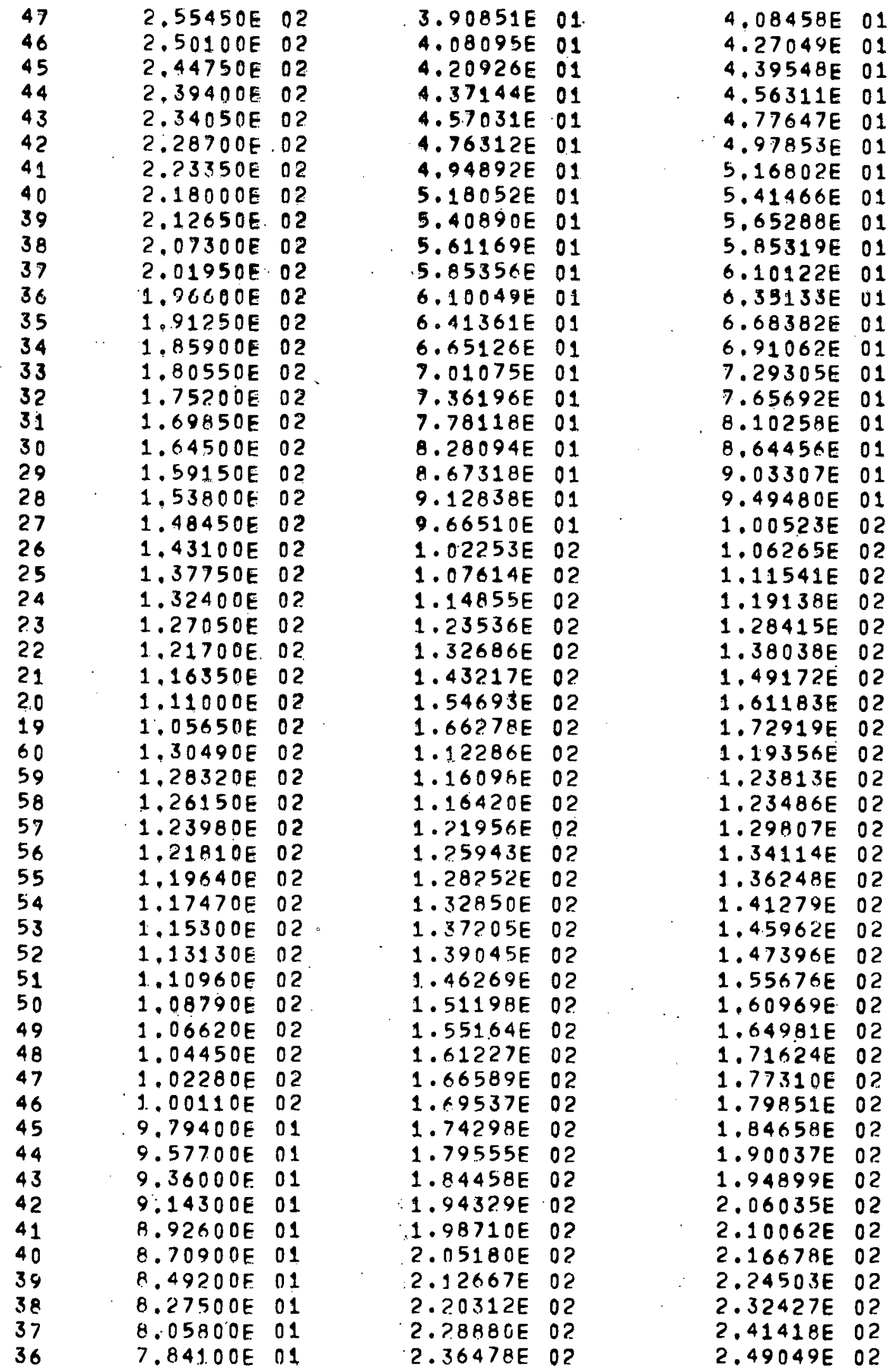


TABLE D.IV. (Contd.)

$\begin{array}{lll}35 & 7.62400 E & 01 \\ 34 & 7.40700 E & 01 \\ 33 & 7.19000 E & 01 \\ 32 & 6.97300 E & 01 \\ 31 & 6.75600 E & 01 \\ 30 & 6.53900 E & 01 \\ 29 & 6.32200 E & 01 \\ 28 & 6.10500 E & 01 \\ 27 & 5.8800 E & 01 \\ 26 & 5.67100 E & 01 \\ 25 & 5.45400 E & 01 \\ 24 & 5.23700 E & 01 \\ 23 & 5.02000 E & 01 \\ 22 & 4.80300 E & 01 \\ 21 & 4.58000 E & 01 \\ 20 & 4.36900 E & 01 \\ 19 & 4.15200 E & 01 \\ 60 & 7.76900 E & 01 \\ 59 & 7.64000 E & 01 \\ 58 & 7.51100 E & 01 \\ 57 & 7.38200 E & 01 \\ 56 & 7.25300 E & 01 \\ 55 & 7.12400 E & 01 \\ 54 & 6.99500 E & 01 \\ 53 & 6.86600 E & 01 \\ 52 & 6.73700 E & 01 \\ 51 & 6.60100 E & 01 \\ 50 & 6.47900 E & 01 \\ 49 & 6.35000 E & 01 \\ 48 & 6.22100 E & 01 \\ 47 & 6.09200 E & 01 \\ 46 & 5.96300 E & 01 \\ 45 & 5.83400 E & 01 \\ 44 & 5.70500 E & 01 \\ 43 & 5.57600 E & 01 \\ 42 & 5.44700 E & 01 \\ 41 & 5.31400 E & 01 \\ 40 & 5.18900 E & 01 \\ 39 & 5.06000 E & 01 \\ 38 & 4.93100 E & 01 \\ 37 & 4.80200 E & 01 \\ 36 & 4.67300 E & 01 \\ 35 & 4.54400 E & 01 \\ 34 & 4.41500 E & 01 \\ 33 & 4.28600 E & 01 \\ 32 & 4.15700 E & 01 \\ 31 & 4.02800 E & 01 \\ 30 & 3.89900 E & 01 \\ 29 & 3.77000 E & 01 \\ 28 & 3.64100 E & 01 \\ 27 & 3.51200 E & 01 \\ 26 & 3.38300 E & 01 \\ 25 & 3.25400 E & 01 \\ 24 & 3.12500 E & 01 \\ & & \end{array}$

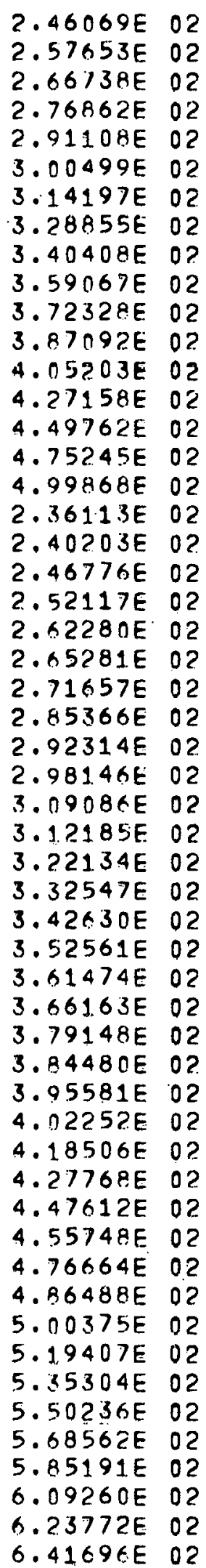

2.59098E 0?

2.71545E O2

2. $80630 E$ O2

$2.90874 E$ O2

$3.06195 E \quad 02$

3.15090 E 02

$3.29274 E$ O2

$3.44428 E$ O2

$3.55337 E$ O2

$3.75038 E \quad 02$

$3.87495 E$ O2

$4.01535 E$ O2

$4.19403 E$ O?

$4.41911 E \quad 02$

$4.64653 E$ O2

$4.90488 E$ O2

$5.14549 E$. 02

$2.47168 E \quad 02$

2.51668E 0 ?

$2.58672 E$ O2

2.64054E 02

$2.75549 E \quad 02$.

$2.77825 E \quad 02$

$2.84369 E \quad 02$

3.00216E 02

$3.07329 E \quad 02$

$3.12941 E \quad 02$

$3.25003 E \quad 02$

$3.26950 E$ O2

$3.37579 E$ O2

$3.48698 E$ O?

3.59283 E 02

$3.69557 E \quad 02$

$3.78409 E \quad 02$

$3.81749 E$ O2

$3.95583 E$ O2

3.99500 E 02

$4.10676 E \quad 02$

$4.16055 E$ 0?

$4.33548 E$ O2

4.41929 E 02

4.63662 E 02

$4.70213 E \quad 02$

$4.92901 E 02$

$5.01158 E$ O?

$5.14390 E$ O2

$5.33942 E \quad 02$

$5.49192 \mathrm{E} \quad 02$

$5.62914 E \quad 02$

5.80666 E 02

$5.95910 E$ O?

6.20294 E 02

$6.32049 E$ O2

$6.47782 E$ O? 
TABLE D.IV. (Contd.)

$\begin{array}{lll}23 & 2.9960 O E & 01 \\ 22 & 2.86700 E & 01 \\ 21 & 2.73800 E & 01 \\ 20 & 2.60900 E & 01 \\ 19 & 2.48000 E & 01 \\ 60 & 4.51700 E & 01 \\ 59 & 4.44220 E & 01 \\ 58 & 4.36740 E & 01 \\ 57 & 4.29260 E & 01 \\ 56 & 4.21780 E & 01 \\ 55 & 4.14300 E & 01 \\ 54 & 4.06820 E & 01 \\ 53 & 3.99340 E & 01 \\ 52 & 3.91860 E & 01 \\ 51 & 3.84380 E & 01 \\ 50 & 3.76900 E & 01 \\ 49 & 3.09420 E & 01 \\ 48 & 3.61940 E & 01 \\ 47 & 3.54460 E & 01 \\ 46 & 3.46980 E & 01 \\ 45 & 3.39500 E & 01 \\ 44 & 3.32020 E & 01 \\ 43 & 3.24540 E & 01 \\ 42 & 3.17060 E & 01 \\ 41 & 3.09580 E & 01 \\ 40 & 3.02100 E & 01 \\ 39 & 2.94620 E & 01 \\ 38 & 2.87140 E & 01 \\ 37 & 2.79660 E & 01 \\ 36 & 2.72180 E & 01 \\ 35 & 2.64700 E & 01 \\ 34 & 2.57220 E & 01 \\ 33 & 2.49740 E & 01 \\ 32 & 2.42260 E & 01 \\ 31 & 2.34780 E & 01 \\ 30 & 2.27300 E & 01 \\ 29 & 2.19820 E & 01 \\ 28 & 2.12340 E & 01 \\ 27 & 2.04860 E & 01 \\ 26 & 1.97380 E & 01 \\ 25 & 1.89900 E & 01 \\ 24 & 1.82470 E & 01 \\ 23 & 1.74940 E & 01 \\ 22 & 1.67460 E & 01 \\ 21 & 1.59980 E & 01 \\ 20 & 1.52500 E & 01 \\ 19 & 1.45020 E & 01 \\ 60 & 2.64500 E & 01 \\ 59 & 2.60140 E & 01 \\ 58 & 2.55780 E & 01 \\ 57 & 2.51420 E & 01 \\ 56 & 2.47060 E & 01 \\ 55 & 2.42700 E & 01 \\ 54 & 2.38340 E & 01\end{array}$

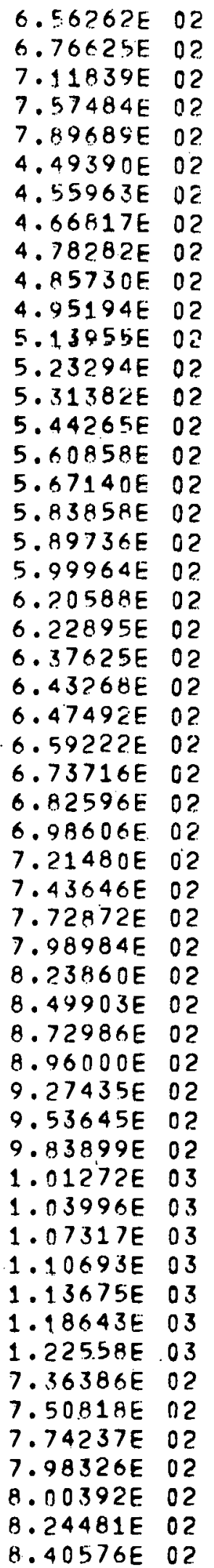

$6.58829 E \quad 02$

$6.76894 \mathrm{E} \quad 02$

$7.13438 E \quad 02$

7.62560 E 02

$7.93540 E \quad 02$

$4.59972 E$ O2

4.67164 E 0 ?

$4.78586 E$ O2

$4.90719 E \quad 02$

$4.97646 E \quad 02$

$5.07082 E \quad 02$

$5.20314 E$ O2

$5.37385 E$ O?

$5.44764 E$ O2

5.58176E 02

5.76208E 02

$5.80926 E \quad 02$

$5.98871 E \quad 02$

$6.02819 E \quad 02$

$6.12216 E \quad 02$

$6.34757 E$ D2

$6.33721 E \quad 02$

$6.48449 E \quad 02$

$6.51412 E \quad 02$

$6.52456 E \quad 02$

$6.62987 E$ O?

$6.76912 E \quad 02$

$6.83510 E \quad 02$

$6.99079 E \quad 02$

$7.23223 E \quad 02$

$7.46206 E \quad 02$

$7.77912 E \quad 02$

$8.05268 E \quad 02$

$8.30648 E \quad 02$

8.57092 E 02

$8.79293 \mathrm{E} 02$

$9.00937 \mathrm{E} \mathrm{O2}$

$9.32834 \mathrm{E} \quad 02$

$9.57469 E \quad 02$

$9.86672 E$ O2

$1.01339 E \quad 03$

$1.03740 E$ O3

$1.06834 E \quad 03$

$1.09919 \mathrm{E} \mathrm{O3}$

.1.12417E 03

$1.17361 E$ OS

$1.20844 E \quad 03$

$7.34824 E \quad 02$

$7.51801 E \quad 02$

$7.78239 E \quad 02$

8. $05392 E 02$

$8.04260 E \quad 02$

$8.31187 E$ O2

$8.47732 E \quad 02$ 
TABLE D.IV. (Contd.)

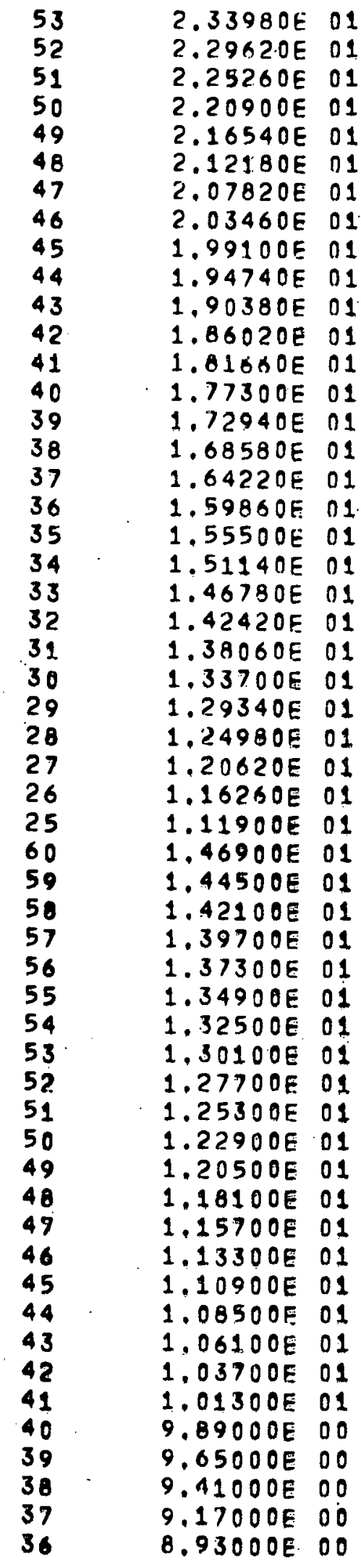

\begin{tabular}{|c|c|}
\hline $\begin{array}{l}8.58335 E \\
8.76174 E \\
8.91035 E \\
9.13112 E \\
9.22501 E \\
9.34921 E \\
9.52197 E \\
9.81678 E \\
9.86802 E \\
1.02259 E \\
1.02428 E \\
1.05118 E \\
1.06079 E \\
1.08351 E \\
1.10003 E \\
1.11723 E \\
1.13871 E \\
1.16232 E \\
1.17243 E \\
1.21747 E \\
1.24714 E \\
1.26745 E \\
1.29792 E \\
1.32413 E \\
1.35906 E \\
1.39718 E \\
1.43465 E \\
1.47390 E \\
1.53104 E \\
1.21934 E \\
1.24915 E \\
1.25775 E \\
1.27591 E \\
1.29678 E \\
1.32084 E \\
1.33945 E \\
1.38690 E \\
1.39624 E \\
1.41481 E \\
1.43591 E \\
1.45363 E \\
1.49359 E \\
1.51016 E \\
1.53199 E \\
1.5620 .6 E \\
1.59345 E \\
1.60309 E \\
1.64335 E \\
1.06392 E \\
1.68313 E \\
1.71793 E \\
1.74763 E \\
1.75875 E \\
1.78614 E\end{array}$ & $\begin{array}{l}02 \\
02 \\
02 \\
02 \\
02 \\
02 \\
02 \\
02 \\
02 \\
03 \\
03 \\
03 \\
03 \\
03 \\
03 \\
03 \\
03 \\
03 \\
03 \\
03 \\
03 \\
03 \\
03 \\
03 \\
03 \\
03 \\
03 \\
03 \\
03 \\
03 \\
03 \\
03 \\
03 \\
03 \\
03 \\
03 \\
03 \\
03 \\
03 \\
03 \\
03 \\
03 \\
03 \\
03 \\
03 \\
03 \\
03 \\
03 \\
03 \\
03 \\
03 \\
03 \\
03 \\
03\end{array}$ \\
\hline
\end{tabular}

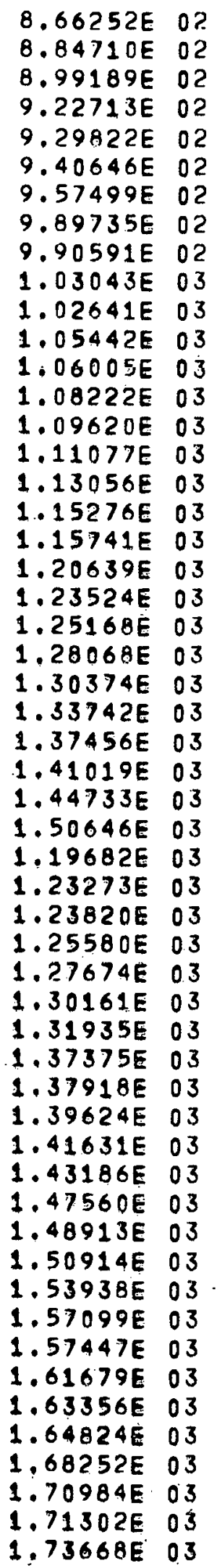


TABLE D.IV. (Contd.)

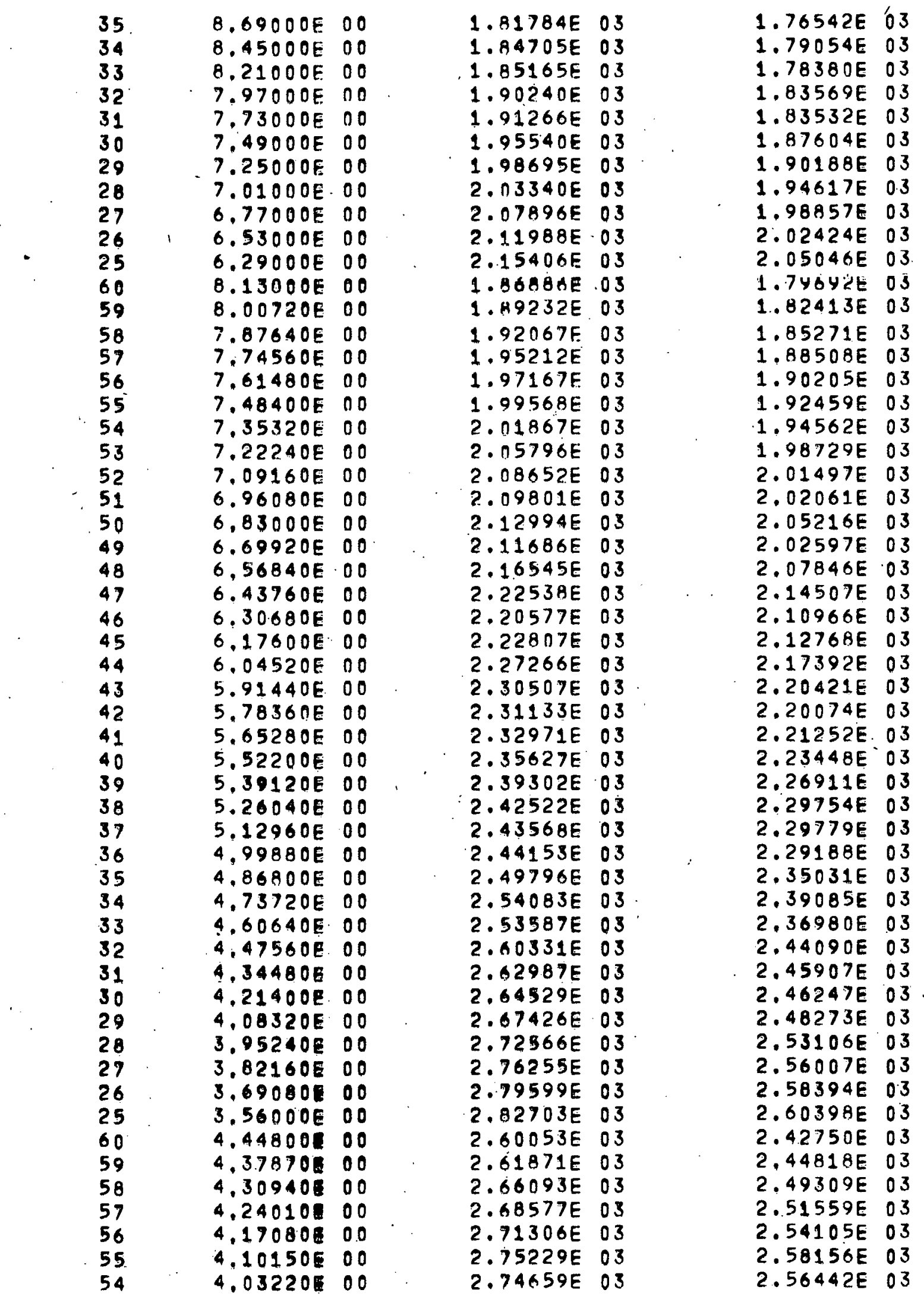


TABLE D.IV. (Contd.)

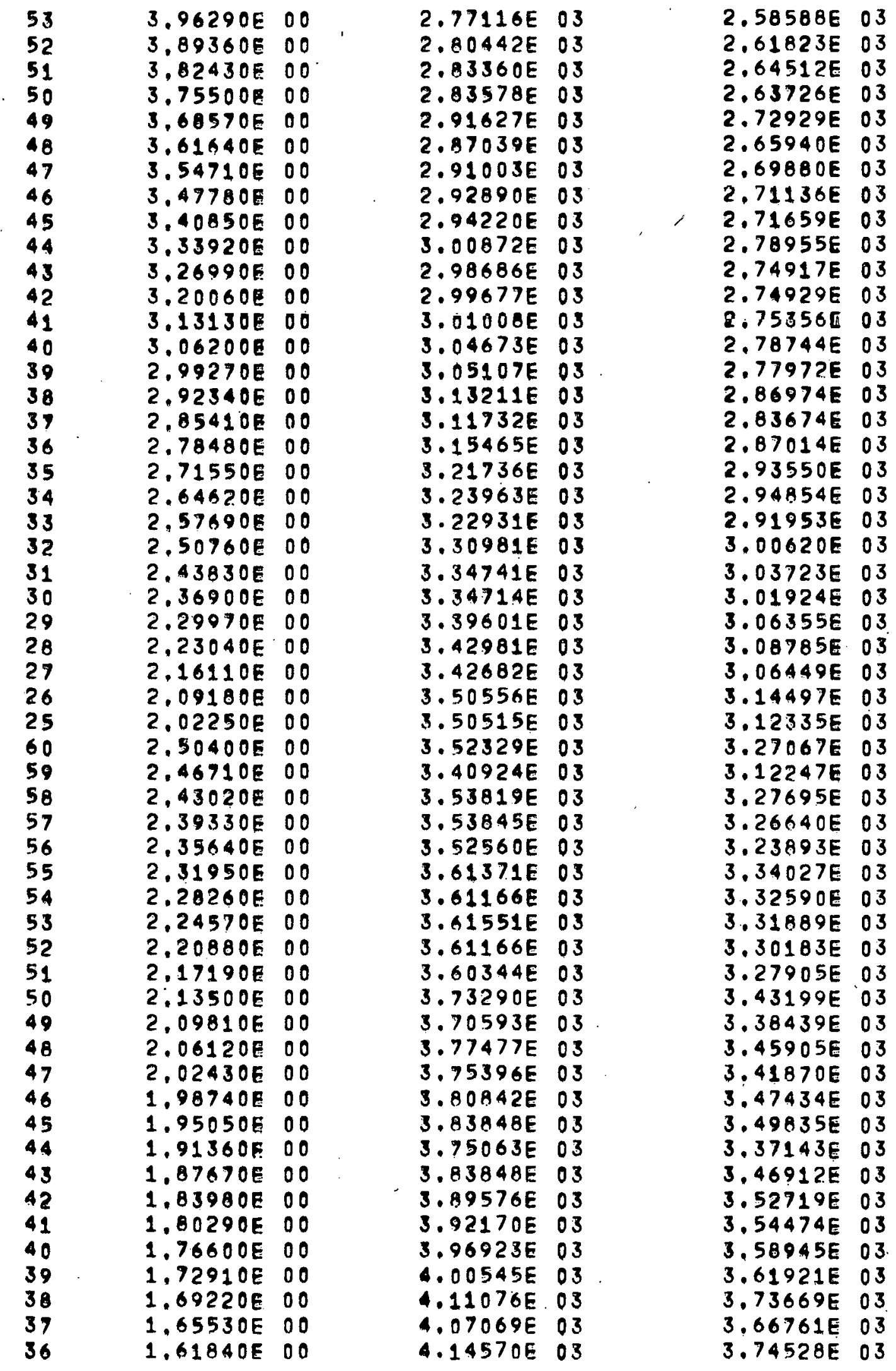


TABLE D.IV. (Contd.)

$\begin{array}{lll}35 & 1.58150 E & O 0 \\ 34 & 1.54460 E & O 0 \\ 33 & 1.50770 E & 00 \\ 32 & 1.47080 E & 00 \\ 31 & 1.43390 E & 00 \\ 30 & 1.39700 E & 00 \\ 29 & 1.36010 E & 00 \\ 28 & 1.32320 E & 00 \\ 27 & 1.28630 E & 00 \\ 26 & 1.24940 E & 00 \\ .25 & 1.21250 E & 00\end{array}$
$4.16265 E$ O3
$4.18063 E 03$
$4.14313 E \quad 03$
$4.22738 F \quad 03$
$4.23971 E$ O3
$4.37252 E \quad 03$
$4.39975 E \quad 03$
$4.39 B 21 E$ O3
$4.45 B 57 E$ O3
4.5990BE O3
$4.66715 E \quad 03$

$\begin{array}{ll}3.74807 E & 03 \\ 3.75167 E & 03 \\ 3.68398 E & 03 \\ 3.77165 E & 03 \\ 3.76669 E & 03 \\ 3.91505 E & 03 \\ 3.92732 E & 03 \\ 3.90213 E & 03 \\ 3.9 .5542 E & 03 \\ 4.11000 E & 03 \\ 4.17036 E & 03\end{array}$
RAW FLUX/LPY.

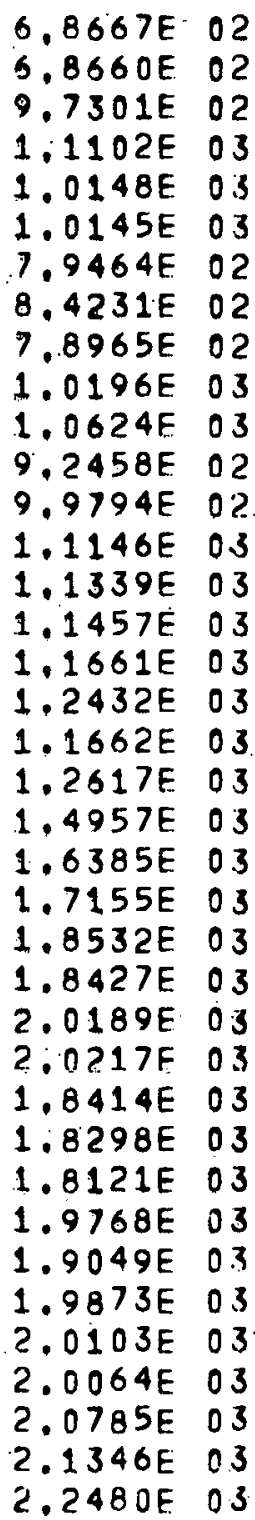

EDIT ENERGY

$2.1783 E$ O3

$2.0746 E$ O3

$1.9758 E$ O 33

$1.8817 E \quad 03$

$1.7921 E$ O3

$1.7068 E \quad 03$

$1.6255 E \quad 03$

$1.5481 E \quad 03$

$1.4744 \mathrm{E} 03$

$1.4042 E \quad 03$

$1.3373 E$ O 3

$1.2736 E$ O 3

$1.2130 E$ O3

$1.1552 E$ O3

$1.1002 E \quad 03$

$1.0478 E$ O3.

$9.9791 E \quad 02$

$9.5039 E \quad 02$

$9.0513 E 02$

$8.6203 E 02$

$8.2098 E \quad 0.2$

$7.8189 E \quad 02$

$7.4465 E \quad 02$

$7.0920 E \quad 02$

$6.7542 E \quad 02$

$6.4326 E$ O2

$6.1263 E \quad 02$

$5.8346 E$ O2

$5.5567 E$ O2

$3.2921 E \quad 02$

$\$ .0401 E 02$

$4.8001 E$ D2

$4.5715 E \quad 02$.

$4.3538 E \quad 02$

$4.1465 E \quad 02$

$3.9491 E \quad 02$.

$3.7610 E$ O2

$3.5819 E$ O2:
FLUX/LTY

$\because 6.8667 E \quad 02$

$6.8660 E$ O2

$9.7301 E \quad 02$

$1.1102 E \quad 03$

$1.0148 E \quad 03$

$1.0145 E \quad 03$

$7.9464 E 02$

$8.4231 E \quad 02$

$7.8965 E \quad 02$

$1.01 .96 E \quad 03$

$1.0624 F \quad 03$

$9.2458 E \quad 02$

$9.9794 E \quad 02$

$1.1146 E \quad 03$

$1.1339 E \quad 03$

$1.1457 E$ O 3

$1.1661 \mathrm{E}$ O3

$1.2432 \mathrm{E} \quad 03$

$1.1662 \mathrm{~F} \quad 03$

$1.2617 E \quad 03$

$1.4957 \mathrm{E} \quad 03$

$1.6385 E \quad 03$

$1.7155 E$ O 3

$1.8532 E \quad 03$

$1.8427 E$ OJ

2.0189. O3

$2.0221 E \quad 03$

$1.8520 E \quad 03$

$1.8982 E \quad 03$

$1.9388 E \quad 03$

$2.1407 E$ OS

$2.0312 E$ O3

$2.1114 E$ O3

$2.0867 E \quad 03$

$2.0670 E$ O3

$2.1303 E$ O3

$2.2002 E \quad 03$

$2.3318 E \quad 03$
FLUX ERROR

$4.3104 E \quad 01$

$4.3919 E \quad 01$

$4.0819 E \quad 01$

$4.2206 E$ OI

$3.9571 E$ O1

$4.1155 E \quad 01$

$3.8311 \mathrm{E} 01$

$4.0068 E \quad 01$

$3.6928 E$ O1

$3.4181 E$ O1

$3.6392 E \quad 01$

$3,9477 \mathrm{E} \quad 01$

$3.1317 E$ O1

$3.4031 E 01$

$3.1611 E 01$

$3.4830 E \quad 01$

$3.2529 E$ O1

$3.0391 E$ O1

$2.7920 E$ O1

$3.1871 E$ O1

$2,9544 E$ OI

$3,5030 E \quad 01$

$3,2938 E$ O1

$3,0816 E$ O1

$2,9006 E 01$

$2,7501 E$ O1

$2.9652 E \quad 01$

$6.9597 E \quad 01$

$6.5943 E \quad 01$

$6.1158 E$ E 1

$5.8255 E$ E

$5,3973 E$ O1

$6,2273 E 01$

$5,8191 E$ E

$5,3748 E$ O 1

$5,0774 E$ O1

$5.9436 E \quad 01$

$5,5393 E$ O
HEAVY RECOIL

$$
\begin{aligned}
& 0.0000 E=01 \\
& 0.0000 E=01 \\
& 0.0000 E=01 \\
& 0.0000 E=01 \\
& 0.0000 E=01 \\
& 0.0000 E=01 \\
& 0.0000 E=01 \\
& 0.0000 E=01 \\
& 0.0000 E=01 \\
& 0.0000 E=01 \\
& 0.0000 E=01 \\
& 0.0000 E=01 \\
& 0.0000 E=01 \\
& 0.0000 E=01 \\
& 0.0000 E=01 \\
& 0.0000 E=01 \\
& 0.0000 E=01 \\
& 0.0000 E=01 \\
& 0.0000 E=01 \\
& 0.0000 E=01 \\
& 0.0000 E=01 \\
& 0.0000 E=01 \\
& 0.0000 E=01 \\
& 0.0000 E=01 \\
& 0.0000 E=01 \\
& 0.0000 E=01 \\
& 0.0000 E=01 \\
& 0.0000 E=01 \\
& 0.0000 E=01 \\
& 0.0000 E=01 \\
& 0.0000 E=01 \\
& 0.0000 E=01 \\
& 0.0000 E=01 \\
& 2.0912 E=02 \\
& 2.1370 E=02 \\
& 2.0461 E=02 \\
& 3.2641 E=02 \\
& 2.8338 E-02 \\
&
\end{aligned}
$$


TABLE D.IV. (Contd.)

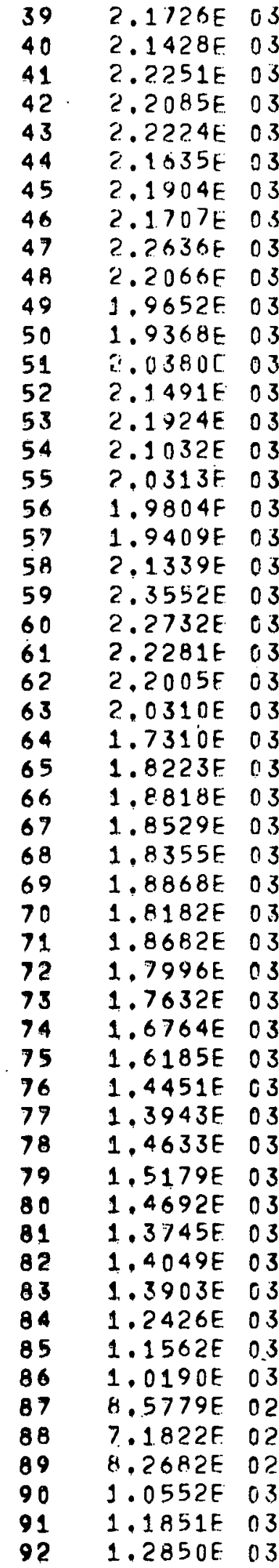

$3.4114 E \quad 02$

$3.2489 E$ O2

3. 0942 E 02

?. $9469 E \quad 02$

?. $.8065 E \quad 02$

$2.6729 \mathrm{E} 02$

$2.5456 E \quad 02$

$2.4244 E \quad 02$

$2.3089 E \quad 02$

$2.1990 E \quad 2$

$2.0943 E \quad 02$

$1.9945 E \quad 0$ ?

$1.0390 \mathrm{E} 02$

$1.8091 \mathrm{E} 02$

$1.7230 E \quad 02$

$1.6409 E \quad 02$

$1.5628 E \quad 02$

$1.4884 \mathrm{E} 02$

$1.4175 E \quad 02$

$1.3500 E \quad 02$

$1.2857 E \quad 02$

$1.2245 E \quad 02$

$1.1662 E \quad 02$

$1.1106 E \quad 02$

$1.0578 \mathrm{E} \quad 02$

$1.0074 E \quad 0$ ?

$9.5941 E 01$

$9.1373 E \quad 01$

8.7022E O1

$8.2878 E$ OI

$7.8931 E$ E

$7.51 .72 \mathrm{E} \quad 01$

$7.1593 E .01$

$6.8184 E 01$

$6.4937 E \quad 01$

$6.1845 E \quad 01$

$5.8900 E$ O1

$5.6095 E \quad 01$

$5.3424 E \quad 01$

$5.0880 E$ OI

$4.8457 E \quad 01$

$4.6149 E$ O1

$4.3952 E \quad 01$

4.1859 E 01

$3.9866 E 01$

$3.7967 E$ O1

$3.6159 E$ O1

$3.4437 E$ O1

$3.2798 E \quad 01$

$3.1236 E$ O1

$2.9748 E$ O1

$2.8332 E \quad 01$

$2.6983 E \quad 01$

2.569RE 01
$2.2042 E \quad 03$

$2.1870 E$ OS

$2.2527 E \quad 0.3$

2.2357E 03

2.? 310 OE 3

$2.1587 E$ O 3

$2.1606 E \quad 03$

$2.1401 E \quad 03$

$2.2356 \mathrm{~F} \quad 03$

$2.1578 \mathrm{~F} 03$

$1.9362 E \quad 03$

$1.9940 E$ O3

?. $0101 E$ US

2.1727E 03

2.?250E 13

$2.0880 F \quad 03$

$1.9779 \mathrm{E} \quad 03$

$1.9020 \mathrm{~F} 03$

$1.8584 \mathrm{E} \quad 03$

2. $0969 E \quad 03$

$2.3542 E \quad 03$

$2.2688 E$ O 3

$2.2173 E$ O 3

$2.3360 E$ O3

$2.1174 \mathrm{E} \quad 3$

$1.7401 E, 03$

$1.8550 \mathrm{E}$ O3

$1.9348 \mathrm{E} \quad 03$

$1.8999 E$ O3

$1.8776 \mathrm{E} \quad 03$

$1.9382 \mathrm{E} \quad 3$

$1.8576 \mathrm{E} \quad 03$

$1.9113 E$ O3

$1.8274 E$ O 3

$1.7692 \mathrm{E} \quad 03$

$1.6702 E \quad 03$

$1.5965 E$ O3.

$1.3735 E$ O 3

$1.3222 \mathrm{~F} \quad 03$

$1.4159 E \quad 0.3$

$1.4938 E \quad 03$

$1.4347 E \quad 03$

$1.3392 E \quad 03$

$1.3698 E \quad 03$

$1.3571 E \quad 03$

$1.1817 \mathrm{E}$ O3

$1.0672 \mathrm{E} \quad 3$

$9.0543 F \quad 02$

$7.1344 E \quad 02$

$5.4954 E \quad 02$

$7.0534 \mathrm{~F} 02$

$1.0100 \mathrm{~F}$ O3

$1.1776 \mathrm{~F} \quad 03$

1.316OE $\cap 3$
$5.2225 E \quad 01$

$3.8402 E \quad 01$

$3.5996 E \quad 01$

$4.2587 E \quad 01$

$3,9465 E \quad 01$

$3,6018 E \quad 01$

$3.6584 E$ O1.

$3,1449 E \quad 01$

$3,1256 E \quad 01$

$2.8848 E \quad 01$

$5,9763 E$ O1

$5,5260 E \quad 01$

5.1553 E 01

$3,7682 E \quad 01$

4.3704E 01

$5,3217 E$ O1

$3.7314 E \quad 01$

$4,5470 E \quad 01$

$4,1659 E \quad 01$

$2.9601 E 01$

$3,6529 E$ O1

$2.9917 E$ O1

$2.7582 E$ OI

$5.5756 E \quad 01$

$6.1154 E \quad 01$

$5.6168 E \quad 01$

$6.4001 E 01$

$5.8723 E$ O1

$5.4213 E \quad 01$

5.0080E 01

$4.6442 E \quad 01$

$5,3880 E \quad 01$

3. $0811 E \quad 01$

$3.4031 E$ O1

$3.2414 E$ O1

$2.9774 \mathrm{E}$ OI

$3.4623 \mathrm{E}$ O1

$2.6869 E$ OL

$2.4644 E$ O1

$2.6954 E \quad 01$

$2.4784 \mathrm{E} \quad 01$

$2.2725 E \quad 01$

$3.7395 E$ O1

2.6889E 01

$2.8246 E \quad 01$

2.5717F 01

$2.5529 E$ O1

2.3? $42 E$ E 1

$2.2583 E$ O1

$2.4455 E$ OI

$2.2459 E$ OI

$2.0767 \mathrm{E} \mathrm{.01}$

$1.9119 E \quad 01$

$3.1491 E$ OI
3. ก034E-02

$? .3790 E=02$

$2.4267 E-02$

$2.2518 E-02$

?. $9571 \mathrm{E}-02$

$3.2679 E-02$

$2.8144 E-02$

3. $0528 \mathrm{E}-02$

$3.2939 E-02$

$3.4523 E-02$

$1.9927 \mathrm{E}-02$

$4.1624 E=02$

$4.16 \mathrm{~b} / E-02$

$3.6353 E-02$

3. $8478 \mathrm{~F}-02$

4.8559E-02

$5.6123 E=02$

3. $1120 E-02$

$6.7471 E-02$

$5.9506 E-02$

$5.7956 \mathrm{E}-02$

$3.4055 E=02$

$3.2605 E-02$

$8.9143 E-03$

1. $0111 E-02$

$1.3630 E-02$

$1.2212 E-02$

$1.2228 E=02$

$1.2374 E=02$

1. $2476 E-02$

$1.2523 E-02$

1. $3571 E-02$

$1.4369 \mathrm{E}=02$

$1.4451 E=02$

$1.4714 \mathrm{E}=02$

$1,6068 E-02$

$1.6734 \mathrm{E}=02$

1. $8913 \mathrm{E}-02$

1. $8869 \mathrm{E}-02$

1. $7861 E=02$

$1.7079 E-02$

$1.8666 E-02$

$1,9501 \mathrm{E}-02$

$1.8053 E-02$

$1.8367 E-02$

$2.2172 E-02$

?. $5724 E-02$

$3.0289 E-02$

$3.5345 E-02$

$4.3299 E-02$

$3.3079 E-02$

?.3436E-02

2. $3192 E-02$

?. 2819E-02 
TABLE D.IV. (Contd.)

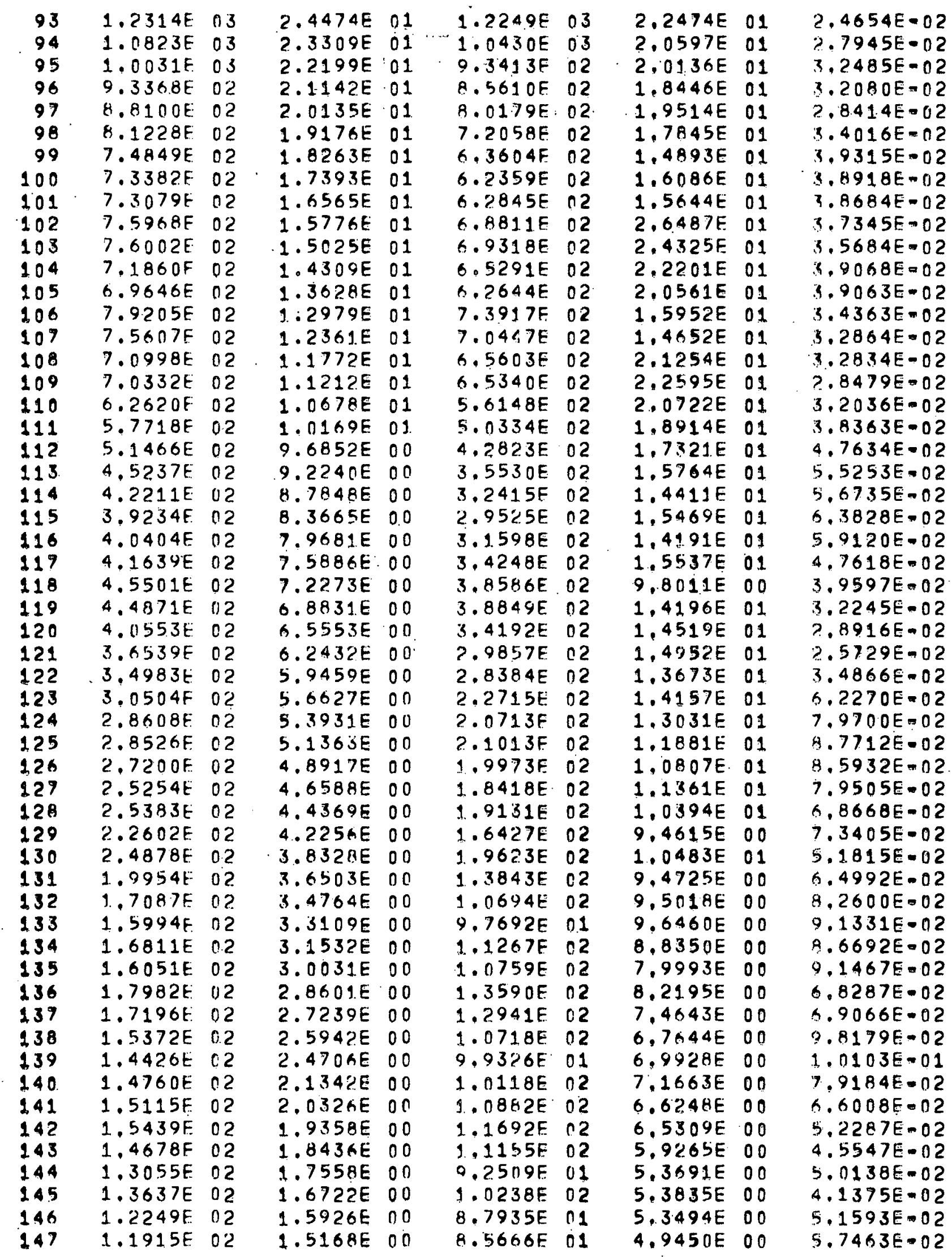




\section{ACKNOWLEDGMENTS}

We gratefully acknowledge the assistance of $\mathrm{Mr}$. R. R. Hopf who helped with the collection and reduction of the experimental data. In addition, we are indebted to Messrs. R. E. Grajek and E. Lewandowski for assistance with the construction of the counters. 
REFERENCES

1. E. F. Bennett, R. Gold, and R. J. Huber, "Spectrum Measurements in a Large Dilute Plutonium-Fueled Fast Reactor," Proceedings of the Intermational Conference on Fast Critical. Experiments and Their Analysi.s, October 10-13, 1966, ANL-7320, pp. 477-480.

2. B. B. Rossi and H. H. Staub, Ionization Chombers and Couriters, McGrawHill Book'Co., New York (1950)。

3. A. G: Engelkemeir and W. F. Libby, End and Wall Corrections for Absolute Beta-Counting in Gas Counters, Rev. Sci. Instr. 21, 550 (1950)。

4. T. H. R. Skylute, P. R. Tunniclitfe, and A. G: Ward, A Proportional Counter for Neutron. Flux Measurements in the Energy Range 0.1 to $1 \mathrm{MeV}$, Rev。Sci. Instr。23, 204 (1952)。

5. R. Batchelor; R. Aves, and T. H. R. Skyrme, Helium-3 FilZed Proportional Counter for Neutron Spectroscopy, Rev. Sci. Instr. 26, 1037 (1955).

6. A. T. G: Ferguson, "Gas-Recoil Counters," Fast Neutron Physics, Part I, J. B. Marion and J。 L. Fowler, Eds., Interscience Publishers Inc。 (1960), p. 179.

7. J: B。 Parker, P。H. White, and R. J. Webster, The Interpretation of Recoil Proton Spectra, Nuc1. Instr. Methods 23, 61 (1962).

8. P. W. Benjamin, C. D. Kemshall, and J. Redfearn, The Use of a Gas-. Filled Spherical Proportional Counter for Neutron Spectrum Measurements in a Zero Power Fast Reactor, AWRE Report No. NR 2/64 (1964).

9. P. W. Benjamin and C. D. Kemsha11, The Analysis of Recoil Proton Spectra, AWRE Report NR 0-7/67 (1967).

10. No L. Snidow and H. D. Warren, Wall Effect Corrections in Proportional Counter Spectrometers, Nucl. Instr. Methods 51, 109 (1967)。

11. R. Gold, An Iterative Unfolding Method for Response Matrices, ANL-6984 (Dec 1964)。

12. A. Spernol and B. Denecke, Precise Absolute Measurement of the Activity of Tritium II, Intern. J. App1. Radiation Isotopes 15; 195 (1963).

13. E. F. Bennett, Fast Neutron Spectroscopy by Proton-Recoil Proportional Counting, Nuc1. Sci. Eng.27, 16 (1967):

14. E. F. Bennett; Neutron Spectmim Measurement in a Fast Critical Assembly, Nucl. Sci. Eng. 27, 28 (1967).

15. P. W. Benjamin, C. D. Kemsha11, and J. Redfearn, A High Resolution Proportional. Counter, AWRE Report No. NR 1/64 (1964).

16. R. Gold, Energy Spectrum of Fast Cosmic Ray Neutrons Near Sea Level, Phys。Rev. 165, 1406 (19.68).

17. R. J. Armani, The Argonne Thermal Source Reactor, ANL-6440 (March 1962)。

18. W. Whaling, "The Energy Loss of Charged Particles in Matter," Handbuch der Physik, Vo1. XXXIV, p. 1.93 (1958). 
19. M. S. Livingston and H. A. Bethe, Nuclear Physics, C. Nuclear Dynamics, Experimental; Rev. Mod. Phys. 9, 245 (1937).

20. J. L. Gammel, "The n-p Total and Differential Cross Sections, in the Energy Range 0-40 MeV," Fast Neutron Physics, Part.II, J. B. Marion and J. L. Fowler, Eds., Interscience Publishers, Inc. (1963), p. 2185.

21. E. F。 Bennett, A Study of the 1/E. SZowing-down Neutron Spectrum Using $4 \pi$-Recoil Proportional Counters, ANL-6897 (Sept 1964).

22. W. C. Davidon; Variable Metric Method for Minimization, ANL-5990 (Nov 1959). 
Torm AEC-426 Rev. $6 / 68$

AECM 3201
U. S. ATONIC ENESGY COMMSSION

RECONMENDATION FOR DISPOSITION OF INFORVATION PRODUC?

(Washington-designated contractors use Form AEC-4.27)

- See Instructions on Reverse Side -

3. AEC Report NC. ANL-7394

\section{Title ANALYSIS AND REDUCTION OF PROTON-RECOIL} DATA

3. Type of Infomation Product:

Q. Scientific and technical report.

$\square$ b. Conference paper (if this box is checked, items 5, 6, and 7 below do not apply.) Title of conference

Date of conference

Exact location

Sponsoring organization

$\square$ c. Other (Specify)

4. Copies Transmitted: (Check one or more applicable boxes)

a. Copies being transmitted for standard distribution by AEC - DTI.

Qb. Copies being transmitted for special distribution by AEC - DTI, per attached Iist.

$\square$ c. A single reproducible copy being transmitted to AEC - DTI.

5. Recommended Distribution: (Check one)

Qa. Normal handling: no restraint on distribution except as may be recuired by the security classification and patent clearance status.

$\square$. Normal handling in the U.S., but there should be no foreign distribution.

C. Make available only to U.S. Government agencies and their contractors.

Dd. Nake available only within $\mathrm{AEC}$ and to AEC contractors.

$\square$ प. Make available oniy within AEC.

पi. Make available only to those listed in item 10., below.

$\square g$. Make available only as specifically approved by the responsible program division.

6. Recomended Announcement:

X. Normal procedure may be followed.

$\square$ b. Recommend following announcement limitations:

7. Reason for Restrictions Recommended in 5. or 6. above:

8. Patent Clearance: (Check a. or b.)

a. AEC patent clearance has been granted by responsible AEC patent group.

$\square$ b. Report has been sent to responsible AEC patent group for clearance.

\section{9.: Defense Information: (For classified information product only.}

?a. does

Rèport

$$
\text { Db. does not }
$$

contain defense information other than "estricred data.

10. Additional infermation or remarks.

11. Submited h:

Alvin Glassner

Name and position (Please print or type)

Chief Editor

organizacion

Technical Publications Department

Argonne National Laboratory

Sigrature

Slecener (D.M)

September 12,1968 


\section{RECEIVEB}

SEP 16931 AH ' 68

D. T. S. EXTENS. HAIL ROOM 